\title{
Electrons
}

and

\section{Chemical Bonding}




\section{Electrons and}

Chemical Bonding

Harry B. Gray

Columbia University

1965

W. A. BENJAMIN, INC. New York Amsterdam 
ELECTRONS AND CHEMTCAL BONDING

Copyright (C) 1964 by W. A. Benjamin, Inc. All rights reserved

Library of Congress Catalog Card Number 64-22275 Manufactured in the United States of America

The manuscript was put into production on January 16, 1964; this volume was published on August 21, 1964; second printing with corrections April 15, 1965

The publisher is pleased to acknowledge the assistance of Lenore Stevens, who copyedited the manuscript, and William Prokos, who produced the illustrations and designed the dust jacket 
To my Students in Chemistry 10 


\section{Preface}

7 HIS BOOK WAS DEVELOPED from my lectures on chemical bonding in Chemistry 10 at Columbia in the spring of 1962, and is mainly intended for the undergraduate student in chemistry who desires an introduction to the modern theories of chemical bonding. The material is designed for a one-semester course in bonding, but it may have greater use as a supplementary text in the undergraduate chemistry curriculum.

The book starts with a discussion of atomic structure and proceeds to the principal subject of chemical bonding. The material in the first chapter is necessarily quite condensed and is intended as a review. (For more details, the student is referred to R. M. Hochstrasser, Behavior of Electrons in Atoms, Benjamin, New York, 1964).

Each chapter in the bonding discussion is devoted to an important family of molecules. Chapters II through VII take up, in order, the principal molecular structures encountered as one proceeds from hydrogen through the second row of the periodic table. Thus, this part of the book discusses bonding in diatomic, linear triatomic, trigonal planar, tetrahedral, trigonal pyramidal, and angular triatomic molecules. Chapters VIII and IX present an introduction to modern ideas of bonding in organic molecules and transition metal complexes. Throughout, our artist has used small dots in drawing the boundary-surface pictures of orbitals. The dots are intended only to give a pleasing three-dimensional effect. Our drawings are not intended to be charge-cloud pictures. Charge-cloud pictures attempt to show the electronic charge density in an orbital as a function of the distance from the nucleus by varying the "dot concentration." 
The discussion of atomic structure does not start with the Schrödinger equation, but with the Bohr theory. I believe most students appreciate the opportunity of learning the development of atomic theory in this century and can make the transition from orbits to orbitals without much difficulty. The student can also calculate several important physical quantities from the simple Bohr theory. At the end of the first chapter, there is a discussion of atomic-term symbols in the Russell-Saunders $L S M_{L} M_{S}$ approximation.

In this book the molecular orbital theory is used to describe bonding in molecules. Where appropriate, the general molccular orbitals are compared with valence-bond and crystal-field descriptions. I have written this book for students who have had no training in group theory. Although symmetry principles are used throughout in the molecular orbital treatment, the formal group-theoretical methods are not employed, and only in Chapter IX are group-theoretical symbols used. Professor Carl Ballhausen and $I$ are publishing an introductory lecture-note volume on molecular orbital theory, which was written at a slightly higher level than the present book. The lecture notes emphasize the application of group theory to electronic structural problems.

The present material includes problems integrated in the text; most of these are accompanied by the worked-out solutions. There are also a substantial number of problems and questions at the end of each chapter.

It is a great pleasure to acknowledge the unfailing support, encouragement, and devotion of the seventy-seven fellows who took the Columbia College course called Chemistry 10 in the spring of 1962. I doubt if I shall ever have the privilege of working with a finer group. The class notes, written by Stephen Steinig and Robert Price, were of considerable help to me in preparing the first draft.

I would like to thank Professors Ralph G. Pearson, John D. Roberts, and Arlen Viste for reading the manuscript and offering many helpful suggestions. Particularly I wish to thank one of my students, James Halper, who critically read the manuscript in every draft. Finally, a large vote of thanks goes to Diane Celeste, 


\section{Contents}

Preface v v

耳 Electrons in Atoms I

1-1 Introductory Remarks I

1-2 Bohr Theory of the Hydrogen Atom (1913) 1

1-3 The Spectrum of the Hydrogen Atom 5

1-4 The Need to Modify the Bohr Theory 9

1-5 Electron Waves 9

1-6 The Lncertainty Principle 11

1-7 The Wave Function $\quad 12$

1-8 The Schrödinger Wave Equation 13

1-9 The Normalization Constant 13

1-10 The Radial Part of the Wave Function 13

1-11 The Angular Part of the Wave Function 14

1-12 Orbitals 14

1-13 Electron Spin 17

1-14 The Theory of Many-Electron Atoms 20

1-15 Russell-Saunders Terms $\quad 22$

1-16 Ionization Potentials 27

1-17 Electron Affinities

II Diatomic Molecules $\quad 36$

2-1 Covalent Bonding $\quad 36$

2-2 Molecular-Orbital Theory 38 
2-3 Bonding and Antibonding Molecular Orbitals $\quad 39$

2-4 Molecular-Orbital Energy Levels $\$ 2$

2-5 The Hydrogen Molecule 46

2-6 Bond Lengths of $\mathrm{H}_{2}{ }^{+}$and $\mathrm{H}_{2}$

2-7 Bond Energies of $\mathrm{H}_{2}{ }^{+}$and $\mathrm{H}_{2}$

2-8 Properties of $\mathrm{H}_{2}{ }^{+}$and $\mathrm{H}_{2}$ in a Magnetic Field 48

2-9 Second-Row Homonuclear Diatomic Molecules 49

2-10 Other A. Molecules 58

2-11 Term Symbols for Linear Molecules 60

2-12 Heteronuclear Diatomic Molecules 62

2-13 Molecular-Orbital Energy-Level Scheme for LiH 67

2-14 Ground State of LiH 68

2-15 Dipole Moments 69

2-16 Electronegativity $\quad 69$

2-17 Ionic Bonding 73

2-1.8 Simple Ionic Model for the Alkali Halides 75

2-19 General AB Molecules $\quad 78$

III Linear Triatomic Molecules $\quad 87$

3-1 $\mathrm{BeH}_{2}$

3-2 Energy Levels for $\mathrm{BeH}_{2} \quad 89$

3-3 Valence-Bond Theory for $\mathrm{BeH}_{2}$

3-4 Linear Triatomic Molecules with $\pi$ Bonding 95

3-5 Bond Properties of $\mathrm{CO}_{2} \quad 100$

3-6 Ionic Triatomic Molecules: The Alkaline Earth Halides

IV Trigonal-Planar Molecules 106

$\begin{array}{lll}4-1 & \mathrm{BF}_{3} & 106\end{array}$

4-2 $\sigma$ Molecular Orbitals 106

$\begin{array}{lll}4-3 & \pi \text { Molecular Orbitals } & 109\end{array}$

4-4 Energy Levels for $\mathrm{BF}_{3}$

4-5 Equivalence of $\sigma_{x}$ and $\sigma_{y}$ Orbitals 112

4-6 Ground State of $\mathrm{BF}_{3}$

4-7 Valence Bonds for $\mathrm{BF}_{3}$

4-8 Other Trigonal-Planar Molecules 117 


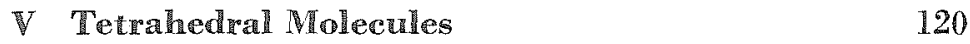

$\begin{array}{llll}5-1 & \mathrm{CH}_{4} & 120\end{array}$

5-2 Ground State of $\mathrm{CH}_{4}$

5-3 The Tetrahedral Angle 122

5-4 Valence Bonds for $\mathrm{CH}_{4}$

5-5 Other Tetrahedral Molecules 127

VI Trigonal-Pyramidal Molecules 129

$\begin{array}{lll}6-1 & \mathrm{NH}_{3} & 129\end{array}$

6-2 Overlap in $\sigma_{x}, \sigma_{y}$, and $\sigma_{z} \quad 130$

6-3 The Interelectronic Pepulsions and $\mathbf{H}-\mathrm{N}-\mathrm{H}$ Bond Angle in $\mathrm{NH}_{3}$

6-4 Bond Angles of Other Trigonal-Pyramidal Molecules

6-5 Ground State of $\mathrm{NH}_{3}$

VIT Angular Triatomic Molecules 141

$\begin{array}{lll}7-1 & \mathrm{H}_{2} \mathrm{O} & 141\end{array}$

7-2 Ground State of $\mathrm{H}_{2} \mathrm{O}$

7-3 Angular Triatomic Molecules with $\pi$ Bonding: $\mathrm{NO}_{2}$

$7-4 \quad \sigma$ Orbitals

$7-5 \quad \pi$ Orbitals 148

$7-6$ Ground State of $\mathrm{NO}_{2}$

VIII Bonding in Organic Molecules 155

8-1 Introduction 155

$\begin{array}{lll}\text { 8-2 } & \mathrm{C}_{2} \mathrm{H}_{4} & 156\end{array}$

8-3 Energy Levels in $\mathrm{C}_{2} \mathrm{H}_{4} \quad 159$

8-4. Ground State of $\mathrm{C}_{2} \mathrm{H}_{4}$

8-5 Bent-Bond Picture of $\mathrm{C}_{2} \mathrm{H}_{4} \quad 160$

8-6 Bond Properties of the $\mathrm{C}=\mathrm{C}$ Group 162

8-7 The Value of $\beta_{c c}$ in $\mathrm{C}_{2} \mathrm{H}_{4}$

8-8 $\mathrm{H}_{2} \mathrm{CO}$

8-9 Ground State of $\mathrm{H}_{2} \mathrm{CO}$ 
8-10 the $n \rightarrow \pi^{*}$ Transition Exhibited by the Carbonyl Group

8-11 $\mathrm{C}_{2} \mathrm{H}_{2}$

8-12 Ground State of $\mathrm{C}_{2} \mathrm{H}_{2}$

8-13 CH. CN

$8-14 \mathrm{C}_{6} \mathrm{H}_{6}$

8-15 Molecular-Orbital Energies in $\mathrm{C}_{6} \mathrm{H}_{6}$

8-16 Ground State of $\mathrm{C}_{6} \mathrm{H}_{6}$

8-17 Riesonance Energy in $\mathrm{C}_{6} \mathrm{H}_{6}$

IX Bonds Involving $d$ Valence Orbilals 176

9-1 Introduction 176

9-2 The Octahedral Complex $\mathrm{Ti}\left(\mathrm{H}_{2} \mathrm{O}\right)_{6}{ }^{3+} \quad 176$

9-3 Energy Levels in $\mathrm{Ti}\left(\mathrm{H}_{2} \mathrm{O}\right)_{6}{ }^{3+} \quad 179$

9-4 Ground State of $\mathrm{Ti}\left(\mathrm{H}_{2} \mathrm{O}\right)_{6}{ }^{3+} \quad 181$

9-5 The Electronic Spectrum of $\mathrm{Ti}\left(\mathrm{H}_{2} \mathrm{O}\right)_{6}{ }^{3+} \quad 183$

9-6 Valence-Bond Theory for $\left.\mathrm{Ti}\left(\mathrm{H}_{2} \mathrm{O}\right)_{6}{ }^{3+} \quad\right\rfloor 84$

9-7 Crystal-Field Theory for $\mathrm{Ti}\left(\mathrm{H}_{2} \mathrm{O}\right)_{6}{ }^{3+} \quad 186$

9-8 Relationship of the General Molecular-Orbital Treatment to the Valence-Bond and CrystalField Theories 187

9-9 Types of $\pi$ Bonding in Metal Complexes 188

9-10 Square-Planar Complexes 189

9-11 Tetrahedral Complexes 194

9-12 The Value of $\Delta \quad 197$

9-13 The Magnetic Properties of Complexes: Weakand Strong-Field Ligands 200

9-14 The Electronic Spectra of Octahedral Complexes 201

Suggested Reading 212

Appendix: Atomic Orbital Ionization Energies 217

Index 


\section{Physical Constants}

Planck's constant, $h=6.6256 \times 10^{-2 \pi} \mathrm{erg}-\mathrm{sec}$

Velocity of light, $c \quad=2.997925 \times 10^{10} \mathrm{~cm} \mathrm{sec}^{-1}$

Electron rest mass, $m_{e}=9.1091 \times 10^{-28} \mathrm{~g}$

Electronic charge, $e=4.80298 \times 10^{-10} \mathrm{esu}\left(\mathrm{cm}^{3 / 2} \mathrm{sec}^{-1}\right)$

Bohr radius, $a_{0} \quad=0.529167 \mathrm{~A}$

Avogadro's number, $N=6.0247 \times 10^{23} \mathrm{~mole}^{-1}$ (physical scale)

\section{Conversion Factors}

Energy

1 electron volt $(\mathrm{eV})=8066 \mathrm{~cm}^{-1}=23.069 \mathrm{kcal} \mathrm{mole}^{-1}$

1 atomic unit $(\mathrm{au})=27.2 \mathrm{leV}=4.3592 \times 10^{-11} \mathrm{ergs}$

$=2.1947 \times 10^{5} \mathrm{~cm}^{-1}=627.71 \mathrm{kcal} \mathrm{mole}{ }^{-1}$

Length

1 Angstrom (A) $=10^{-8} \mathrm{~cm}$

a Values recommended by the National Bureau of Standards; see J. Chem. Educ., 40, 642 (1963). 


\section{I}

\section{Electrons in Atoms}

\section{1-1 INTRODUCTORY REMARKS}

7 he main purpose of this book is the discussion of bonding in I several important classes of molecules. Before starting this discussion, we shall review briefly the pertinent details of atomic structure. Since in our opinion the modern theories of atomic structure began with the ideas of Niels Bohr, we start with the Bohr theory of the hydrogen atom.

\section{1-2 BOHR THEORY OF THE HYDROGEN ATOM (1913)}

Bohr pictured the electron in a hydrogen atom moving in a circular orbit about the proton (see Fig. 1-1). Note that in Fig. 1-1, $m_{e}$ represents the mass of the electron, $m_{n}$ the mass of the nucleus, $r$ the radius of the circular orbit, and $v$ the linear velocity of the electron.

For a stable orbit, the following condition must be met: the centrifugal force exerted by the moving electron must equal the combined forces of attraction between the nucleus and the electron:

$$
\text { centrifugal force }=\frac{m_{e} v^{2}}{r}
$$

There are two attractive forces tending to keep the electron in orbit: the electric force of attraction between the proton and the electron, 


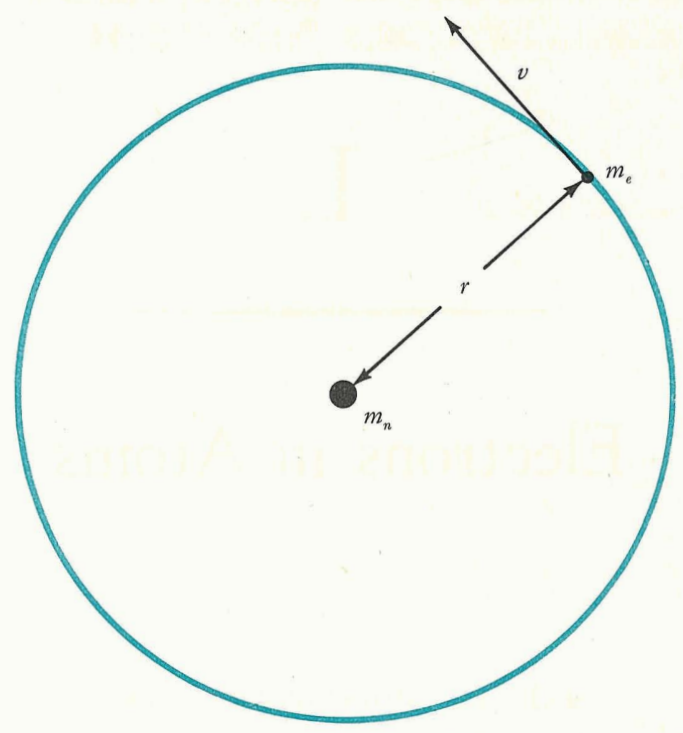

Figure 1-1 Bohr's picture of the hydrogen atom.

and the gravitational force of attraction. Of these, the electric force greatly predominates and we may neglect the gravitational force:

$$
\text { electric force of attraction }=\frac{e^{2}}{r^{2}}
$$

Equating (1-1) and (1-2), we have the condition for a stable orbit, which is

$$
\frac{m_{e} v^{2}}{r}=\frac{e^{2}}{r^{2}}
$$

We are now able to calculate the energy of an electron moving in one of the Bohr orbits. The total energy is the sum of the kinetic energy $T$ and the potential energy $V$; thus

$$
E=T+V
$$

where $T$ is the energy due to motion

$$
T=\frac{1}{2} m_{e} v^{2}
$$


and $V$ is the energy due to electric attraction.

$$
V=\int_{\infty}^{r} \frac{e^{2}}{r^{2}} d r=\frac{-e^{2}}{r}
$$

Thus the total energy is

$$
E=\frac{1}{2} m_{e} v^{2}-e^{2} / r
$$

However, the condition for a stable orbit is

$$
\frac{m_{e} v^{2}}{r}=\frac{e^{2}}{r^{2}} \quad \text { or } \quad m_{e} v^{2}=\frac{e^{2}}{r}
$$

Thus, substituting for $m_{e} v^{2}$ in Eq. (1-7), we have

$$
E=\frac{1}{2} e^{2}-\frac{e^{2}}{r}=-\frac{1 e^{2}}{2 r}
$$

Now we need only specify the orbit radius $r$ and we can calculate the energy. According to Eq. (1-9), all energies are allowed from zero $(r=\infty)$ to infinity $(r=0)$.

At this point Bohr made a novel assumption-that the angular momentum of the system, equal to $m_{e} v r$, can only have certain discrete values, or quanta. The result is that only certain electron orbits are allowed. According to the theory, the quantum unit of angular momentum is $b / 2 \pi$ ( $b$ is a constant, named after Max Planck, which is defined on page 5). Thus, in mathematical terms, Bobr's assumption was

$$
m_{e} v r=n\left(\frac{b}{2 \pi}\right)
$$

with $n=1,2,3 \ldots$ (all integers to $\infty$ ). Solving for $v$ in Eq. (1-10), we have

$$
v=n\left(\frac{b}{2 \pi}\right) \frac{1}{m_{e} r}
$$

Substituting the value of $v$ from Eq. (1-11) in the condition for a stable orbit [Eq. (1-8)], we obtain

$$
\frac{m_{e} n^{2} b^{2}}{4 \pi^{2} m_{e}^{2} r^{2}}=\frac{e^{2}}{r}
$$


or

$$
r=\frac{n^{2} b^{2}}{4 \pi^{2} m_{e} e^{2}}
$$

Equation (1-13) gives the radius of the allowable electron orbits for the hydrogen atom in terms of the quantum number, $n$. The energy associated with each allowable orbit may now be calculated by substituting the value of $r$ from Eq. $(1-13)$ in the energy expression [Eq. (1-9)], giving

$$
E=-\frac{2 \pi^{2} m_{e} e^{4}}{n^{2} b^{2}}
$$

\section{PROBLEMS}

1-1. Calculate the radius of the first Bohr orbit.

Solution. The radius of the first Bohr orbit may be obtained directly from Eq. (1-13)

$$
r=\frac{n^{2} b^{2}}{4 \pi^{2} m_{\ell} e^{2}}
$$

Substituting $n=1$ and the values of the constants, we obtain

$$
\begin{aligned}
r & =\frac{(1)^{2}\left(6.6238 \times 10^{-27} \mathrm{erg}-\mathrm{sec}\right)^{2}}{4(3.1416)^{2}\left(9.1072 \times 10^{-28} \mathrm{~g}\right)\left(4.8022 \times 10^{-10} \mathrm{abs} \mathrm{esu}\right)^{2}} \\
& =0.529 \times 10^{-8} \mathrm{~cm}=0.529 \mathrm{~A}
\end{aligned}
$$

The Bohr radius for $n=1$ is designated $a_{0}$.

I-2. Calculate the velocity of an electron in the first Bohr orbit of the hydrogen atom.

Solution. From Eq. (1-11),

$$
v=n\left(\frac{h}{2 \pi}\right) \frac{1}{m_{e} r}
$$

Substituting $n=1$ and $r=a_{0}=0.529 \times 10^{-8} \mathrm{~cm}$, we obtain

$$
\begin{aligned}
v & =(1) \frac{\left(6.6238 \times 10^{-27} \mathrm{erg}^{-\mathrm{sec})}\right.}{2(3.1416)} \\
\times \frac{1}{\left(9.1072 \times 10^{-28} \mathrm{~g}\right)\left(0.529 \times 10^{-8} \mathrm{~cm}\right)} & \\
& =2.188 \times 10^{8} \mathrm{~cm} \mathrm{sec}^{-1}
\end{aligned}
$$




\section{1-3 THE SPECTRUM OF THE HYDROGEN ATOM}

The most stable state of an atom has the lowest energy and this is called the ground state. From Eq. (1-14) it is clear that the most stable electronic state of the hydrogen atom occurs when $n=1$. States that have $n>1$ are less stable than the ground state and understandably are called excited states. The electron in the hydrogen atom may jump from the $n=1$ level to another $n$ level if the correct amount of energy is supplied. If the energy supplied is light energy, light is absorbed by the atom at the light frequency exactly equivalent to the energy required to perform the quantum jump. On the other hand, light is emitted if an electron falls back from a higher $n$ level to the ground-state $(n=1)$ level.

The light absorbed or emitted at certain characteristic frequencies as a result of the electron changing orbits may be captured as a series of lines on a photographic plate. The lines resulting from light absorption constitute an absorption spectrum, and the lines resulting from emission constitute an emission spectrum. The frequency $\nu$ of light absorbed or emitted is related to energy $E$ by the equation deduced by Planck and Einstein,

$$
E=b \nu
$$

where $h$ is called Planck's constant and is equal to $6.625 \times 10^{-27}$ erg-sec.

It was known a long time before the Bohr theory that the positions of the emission lines in the spectrum of the hydrogen atom could be described by a very simple equation

$$
\nu_{\mathrm{H}}=R_{H}\left(\frac{1}{n^{2}}-\frac{1}{m^{2}}\right)
$$

where $n$ and $m$ are integers, and where $R_{\mathrm{II}}$ is a constant, called the $R y d b e r g$ constant after the man who first discovered the empirical correlation.

This equation can be obtained directly from the Bohr theory as follows: The transition energy $\left(E_{\mathrm{H}}\right)$ of any electron jump in the hydrogen atom is the energy difference between an initial state $I$ and a final state II. That is,

$$
E_{\mathrm{II}}=E_{\mathrm{II}}-E_{\mathrm{I}}
$$


or, from Eq. (1-14),

$$
\begin{aligned}
E_{\mathrm{II}} & =-\frac{2 \pi^{2} m_{e} e^{4}}{n_{\mathrm{II}}^{2} b^{2}}-\left(-\frac{2 \pi^{2} m_{e} e^{4}}{n_{\mathrm{I}}^{2} b^{2}}\right) \\
& =\frac{2 \pi^{2} m_{e} e^{4}}{b^{2}}\left(\frac{1}{n_{\mathrm{I}}^{2}}-\frac{1}{n_{\mathrm{II}}^{2}}\right)
\end{aligned}
$$

Replacing $E_{\mathrm{H}}$ with its equivalent frequency of light from Eq. (1-15), we have

$$
\nu_{\mathrm{H}}=\frac{2 \pi^{2} m_{e} e^{4}}{b^{3}}\left(\frac{1}{n_{\mathrm{I}}^{2}}-\frac{1}{n_{\mathrm{II}^{2}}}\right)
$$

Equation (1-20) is equivalent to the experimental result, Eq. (1-16), with $n_{\mathrm{I}}=n, n_{\mathrm{II}}=m$, and $R_{\mathrm{H}}=\left(2 \pi^{2} m_{e} e^{4}\right) / b^{3}$. Using the value of $9.1085 \times 10^{-28} \mathrm{~g}$ for the rest mass of the electron, the Bohr-theory value of the Rydberg constant is

$$
\begin{array}{r}
R_{\mathrm{II}}=\frac{2 \pi^{2} m_{\theta^{4}} e^{4}}{b^{3}}=\frac{2(3.1416)^{2}\left(9.1085 \times 10^{-28}\right)\left(4.8029 \times 10^{-10}\right)^{4}}{\left(6.6252 \times 10^{-27}\right)^{3}} \\
=3.2898 \times 10^{15} \mathrm{cycles} / \mathrm{sec}
\end{array}
$$

It is common practice to express $R_{H}$ in wave numbers $\bar{\nu}$ rather than in frequency. Wave numbers and frequency are related by the equation

$$
\nu=c \bar{\nu}
$$

where $c$ is the velocity of light. Thus

$$
R_{\mathrm{H}}=\frac{3.2898 \times 10^{15} \mathrm{cycles} / \mathrm{sec}}{2.9979 \times 10^{10} \mathrm{~cm} / \mathrm{sec}}=109,737 \mathrm{~cm}^{-1}
$$

The accurately known experimental value of $R_{\mathrm{H}}$ is $109,677.581 \mathrm{~cm}^{-1}$. This remarkable agreement of theory and experiment was a great triumph for the Bohr theory.

PROBLEMS

1-3. Calculate the ionization potential of the hydrogen atom. Solution. The ionization potential (IP) of an atom or molecule is the energy needed to completely remove an electron from the atom or molecule in its ground state, forming a positive ion. For the hydrogen atom, the process is 


$$
\mathrm{H} \rightarrow \mathrm{H}^{+}+e \quad E=\mathrm{IP}
$$

We may start with Eq. (1-19),

$$
E_{\mathrm{H}}=\frac{2 \pi^{2} m_{c} e^{4}}{b^{2}}\left(\frac{1}{n_{\mathrm{r}}^{2}}-\frac{1}{n_{\mathrm{II}}^{2}}\right)
$$

For the ground state, $n_{\mathrm{r}}=1$; for the state in which the electron is completely removed from the atom, $n_{\mathrm{II}}=\infty$. Thus,

$$
\mathrm{IP}=\frac{2 \pi^{2} m_{e} e^{4}}{b^{2}}
$$

Recall that

$$
a_{0}=\frac{b^{2}}{4 \pi^{2} m_{e} e^{2}}
$$

and therefore

$$
\frac{1}{2 a_{0}}=\frac{2 \pi^{2} m_{\epsilon} e^{2}}{b^{2}}
$$

Then

$$
\mathrm{IP}=\frac{e^{2}}{2 a_{0}}=\frac{\left(4.8022 \times 10^{-10} \mathrm{abs} \mathrm{esu}\right)^{2}}{2\left(0.529 \times 10^{-8} \mathrm{~cm}\right)}=2.179 \times 10^{-11} \mathrm{erg}
$$

Ionization potentials are usually expressed in electron volts. Since $1 \mathrm{erg}=6.2419 \times 10^{11} \mathrm{eV}$, we calculate

$$
\text { IP }=2.179 \times 10^{-11} \mathrm{erg}=13.60 \mathrm{eV}
$$

The experimental value of the IP of the hydrogen atom is $13.595 \mathrm{eV}$. 1-4. Calculate the third ionization potential of the lithium atom. Solution. The lithium atom is composed of a nucleus of charge $+3(Z=3)$ and three electrons. The first ionization potential IP 1 of an atom with more than one electron is the energy required to remove one electron; for lithium,

$$
\mathrm{Li} \rightarrow \mathrm{Li}^{+} \quad E=\mathrm{IP}_{1}
$$

The energy needed to remove an electron from the unipositive ion $\mathrm{Li}^{+}$is defined as the second ionization potential $\mathrm{IP}_{2}$ of lithium,

$$
\mathrm{Li}^{+} \rightarrow \mathrm{Li}^{2+} \quad E=\mathrm{IP}_{2}
$$

and the third ionization potential $\mathrm{IP}_{3}$ of lithium is therefore the energy required to remove the one remaining electron in $\mathrm{Li}^{2+}$. 
The problem of one electron moving around a nucleus of charge +3 (or $+Z$ ) is very similar to the hydrogen atom problem. Since the attractive force is $Z e^{2} / r^{2}$, the condition for a stable orbit is

$$
\frac{m_{e} v^{2}}{r}=\frac{Z e^{2}}{r^{2}}
$$

Carrying this condition through as in the hydrogen atom case and again making the quantum assumption

$$
m_{e} v r=n\left(\frac{b}{2 \pi}\right)
$$

we find

$$
r=\frac{n^{2} b^{2}}{4 \pi^{2} m_{e} Z \vartheta^{2}}
$$

and

$$
E=-\frac{2 \pi^{2} m_{e} Z^{2} e^{4}}{n^{2} b^{2}}
$$

Thus Eq. (1-19) gives, for the general case of nuclear charge $Z$,

$$
E=\frac{2 \pi^{2} m_{8} Z^{2} e^{4}}{b^{2}}\left(\frac{1}{n_{\mathrm{I}}^{2}}-\frac{1}{n_{\mathrm{II}}^{2}}\right)
$$

or simply $E=Z^{2} E_{\mathrm{H}}$. For lithium, $Z=3$ and $\mathrm{IP}_{3}=(3)^{2}(2.179 \times$ $\left.10^{-11} \mathrm{erg}\right)=1.961 \times 10^{-10} \mathrm{erg}=122.4 \mathrm{eV}$.

1-5. The Lyman series of emission spectral lines arises from transitions in which the excited electron falls back into the $n=1$ level. Calculate the quantum number $n$ of the initial state for the Lyman line that has $\bar{\nu}=97,492.208 \mathrm{~cm}^{-1}$.

Solution. We use Eq. (1-20)

$$
\nu_{\mathrm{H}}=\frac{2 \pi^{2} m_{e} e^{4}}{b^{3}}\left(\frac{1}{n_{\mathrm{I}}^{2}}-\frac{1}{n_{\mathrm{II}^{2}}}\right)
$$

in which $n_{\mathrm{Ir}}$ is the quantum number of the initial state for an emission line, and $n_{\mathrm{I}}=1$ for the Lyman series. Using the experimental value

$$
R_{\mathrm{H}}=\frac{2 \pi^{2} m_{e^{e^{4}}}}{c b^{3}}=109,677.581 \mathrm{~cm}^{-1}
$$


we have

$$
97,492.208=109,677.581\left(1-\frac{1}{n_{\mathrm{I}^{2}}}\right) \quad \text { or } \quad n_{\mathrm{II}}=3
$$

\section{1-4 THE NEED TO MODIFY THE BOHR THEORY}

The idea of an electron circling the nucleus in a well-defined orbit - just as the moon circles the earth-was easy to grasp, and Bohr's theory gained wide acceptance. Little by little, however, it was realized that this simple theory was not the final answer. One difficulty was the fact that an atom in a magnetic field has a more complicated emission spectrum than the same atom in the absence of a magnetic field. This phenomenon is known as the Zeeman effect and is not explicable by the simple Bohr theory. However, the German physicist Sommerfeld was able to temporarily rescue the simple theory by suggesting elliptical orbits in addition to circular orbits for the electron. The combined Bobr-Sommerfeld theory explained the Zeeman effect very nicely.

More serious was the inability of even the Bohr-Sommerfeld theory to account for the spectral details of the atoms that have several electrons. But these were the 1920s and theoretical physics was enjoying its greatest period. Soon the ideas of de Broglie, Schrödinger, and Heisenberg would put atomic theory on a sound foundation.

\section{1-5 ELECTRON WAVES}

In 1924, the French physicist Louis de Broglie suggested that electrons travel in waves, analogous to light waves. The smallest units of light (light quanta) are called photons. The mass of a photon is given by the Einstein equation of mass-energy equivalence

$$
E=m c^{2}
$$

Recall from Eq. (1-15) that the energy and frequency of light are related by the expression

$$
E=b \nu
$$


Combining Eq. (1-24) and Eq. (1-25), we have

$$
m=\frac{b \nu}{c^{2}}
$$

The momentum $p$ of a photon is

$$
p=m v=m c
$$

Substituting the mass of a photon from Eq. (1-26), we have

$$
p=\frac{b v}{c}
$$

Since frequency $\nu$, wavelength $\lambda$, and velocity $v$ are related by the expression

$$
\lambda=\frac{\nu}{\nu}
$$

we find

$$
\lambda=\frac{b}{p}
$$

Equation (1-30) gives the wavelength of the light waves or electron waves. For an electron traveling in a circular Bohr orbit, there must be an integral number of wavelengths in order to have a standing wave (see Fig. 1-2), or

$$
n \lambda=2 \pi r
$$

Substituting for $\lambda$ from $\mathrm{Eq}$. (1-30), we have

$$
n\left(\frac{b}{p}\right)=2 \pi r
$$

or

$$
n\left(\frac{b}{2 \pi}\right)=r p=\text { angular momentum }
$$

Thus de Broglie waves can be used to explain Bohr's novel postulate [Eq. (1-10)]. 


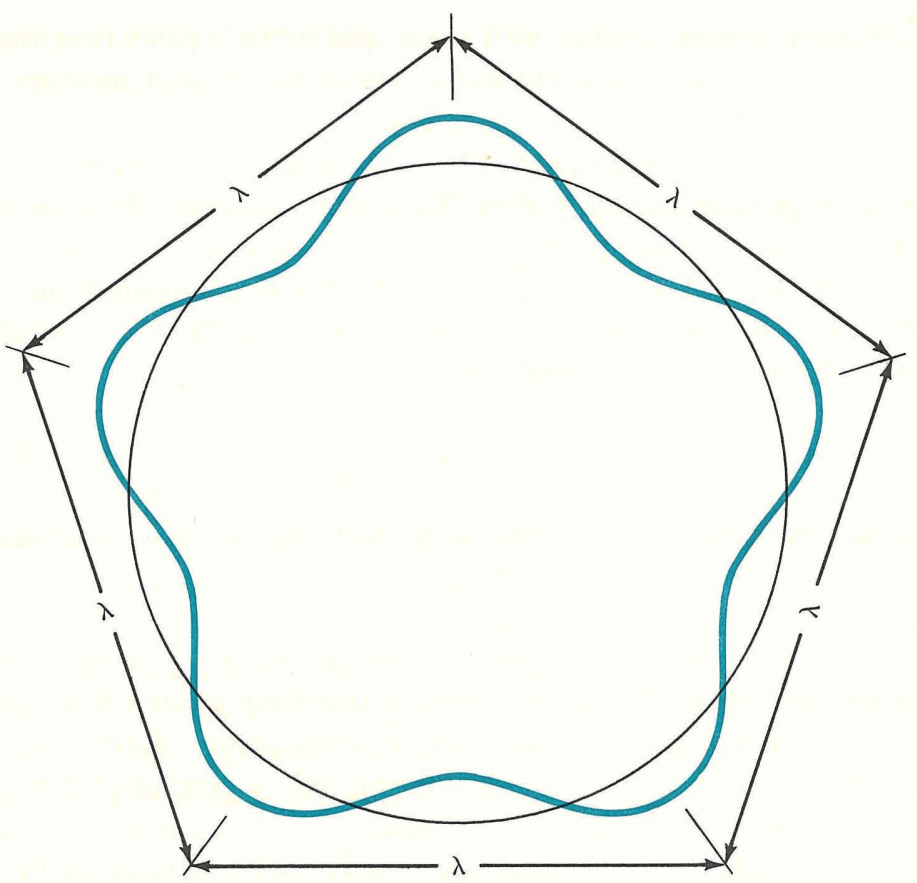

Figure 1-2 A standing electron wave with $n=5$.

\section{1-6 THE UNCERTAINTY PRINCIPLE}

In 1927, Davisson and Germer demonstrated that electrons are diffracted by crystals in a manner similar to the diffraction of $\mathrm{X}$ rays. These electron-diffraction experiments substantiated de Broglie's suggestion that an electron has wave properties such as wavelength, frequency, phase, and interference. In seemingly direct contradiction, however, certain other experiments, particularly those of J. J. Thomson, showed that an electron is a particle with mass, energy, and momentum.

As an attempt at an explanation of the above situation, Bohr put forward the principle of complementarity, in which he postulated that 
an electron cannot exhibit both wave and particle properties simultaneously, but that these properties are in fact complementary descriptions of the behavior of electrons.

A consequence of the apparently dual nature of an electron is the uncertainty principle, developed by Werner Heisenberg. The essential idea of the uncertainty principle is that it is impossible to specify at any given moment both the position and the momentum of an electron. The lower limit of this uncertainty is Planck's constant divided by $4 \pi$. In equational form,

$$
\left(\Delta p_{x}\right)(\Delta x) \geq \frac{h}{4 \pi}
$$

Here $\Delta p_{x}$ is the uncertainty in the momentum and $\Delta x$ is the uncertainty in the position. Thus, at any instant, the more accurately it is possible to measure the momentum of an electron, the more uncertain the exact position becomes. The uncertainty principle means that we cannot think of an electron as traveling around from point to point, with a certain momentum at each point. Rather we are forced to consider the electron as having only a certain probability of being found at each fixed point in space. We must also realize that it is not possible to measure simultaneously, and to any desired accuracy, the physical quantities that would allow us to decide whether the electron is a particle or a wave. We thus carry forth the idea that the electron is both a particle and a wave.

\section{1-7 THE WAVE FUNCTION}

Since an electron has wave properties, it is described as a wave function, $\psi$ or $\psi(x, y, z)$, the latter meaning that $\psi$ is a function of coordinates $x, y$, and $z$. The wave function can take on positive, negative, or imaginary values. The probability of finding an electron in any volume element in space is proportional to the square of the absolute value of the wave function, integrated over that volume of space. This is the physical significance of the wave function. Measurements we make of electronic charge density, then, should be related to $|\psi|^{2}$, not $\psi$. Expressed as an equation, we have

$$
\text { probability }(x, y, z) \propto|\psi(x, y, z)|^{2}
$$


By way of further explanation, it should be noted that the probability of finding an electron in any volume element must be real and positive, and $|\psi|^{2}$ always satisfies this requirement.

\section{1-8 THE SCHRO̊DINGER WAVE EQUATION}

In 1926, the Austrian physicist Erwin Schrödinger presented the equation relating the energy of a system to the wave motion. The Scbrodinger equation is commonly written in the form

$$
\mathfrak{H} \psi=E \psi
$$

whete $\mathcal{F}$ is an operator called the Hamiltonian operator (after the English physicist Hamilton) and represents the general form of the kinetic and potential energies of the system; $E$ is the numerical value of the energy for any particular $\psi$. The wave functions that give solutions to Eq. (1-35) are called eigenfunctions; the energies $E$ that result from the solutions are called eigenvalues.

The Schrödinger equation is a complicated differential equation and is capable of exact solution only for very simple systems. Fortunately, one of these systems is the hydrogen atom.

The solution of the Schrödinger equation for the hydrogen atom yields wave functions of the general form

$$
\psi_{n l m_{l}}=[N]\left[R_{n l}(r)\right]\left[\Phi_{l_{m_{l}}}\left(\frac{x, y}{r}, \frac{y}{r}, \frac{z}{r}\right)\right]
$$

We shall now attempt to explain the parts of Eq. (1-36).

\section{1-9 THE NORMALIZATION CONSTANT}

In Eq. (1-36), $N$ is a nomalization constant, fixed so that

$$
\int_{-\infty}^{\infty} \int_{-\infty}^{\infty} \int_{-\infty}^{\infty}|\psi|^{2} d x d y d z=1
$$

That is, the probability of finding the electron somewhere in space must be unity.

\section{1-10 THE RADIAL PART OF THE WAVE FUNCTION}

$R_{n l}(r)$ is the radial part of the wave function. The value of $\left|R_{n l}(r)\right|^{2}$ gives the probability of finding the electron any distance $r$ 
from the nucleus. The two quantum numbers $n$ and $l$ are associated with the radial part of the wave function: $n$ is called the principal quantum number and defines the mean radius for the electron; $\psi_{n l m_{l}}$ can only be an eigenfunction for $n=1,2,3 \ldots$ integers. $l$ is the quantum number which specifies the angular momentum of the electron; $\psi_{n l m_{l}}$ can only be an eigenfunction for $l=0,1,2,3, \ldots$ to $n-1$.

\section{1-11 THE ANGULAR PART OF THE WAVE FUNCTION}

$\Phi_{l m_{l}}(x / r, y / r, z / r)$ is the angular part of the wave function. The quantum numbers $l$ and $m_{l}$ are associated with the angular part of the wave function. $m_{l}$ is called the magnetic quantum number and defines the possible values for the z-axis component of the angular momentum of the electron in a magnetic field. $\psi_{n l m_{l}}$ can only be an eigenfunction for $m_{l}=+l, l-1, l-2, \ldots$ to $-l$.

\section{1-12 ORBITALS}

The hydrogen eigenfunctions $\psi_{n l m_{l}}$ are commonly called orbitals. The orbitals for the hydrogen atom are classified according to their angular distribution, or $l$ value. Each different $l$ value is assigned a letter:

$$
\begin{aligned}
& l=0 \text { is an } s \text { orbital. } \\
& l=1 \text { is a } p \text { orbital. } \\
& l=2 \text { is a } d \text { orbital. } \\
& l=3 \text { is an } f \text { orbital. }
\end{aligned}
$$

The letters $s, p, d$, and $f$ are taken from spectroscopic notation. For $l=4$ or more, alphabetical order is followed, omitting only the letter $j$. Thus, $l=4$ is a $g$ orbital, $l=5$ is an $b$ orbital, etc.

An orbital is completely specified in this shorthand notation by adding the $n$ and $m_{l}$ values. The $n$ value goes in front of the letter for the $l$ value. The $m_{l}$ value is indicated as a subscript, the total shorthand being $n l_{m_{l}}$. Now for $m_{l} \neq 0$, the $n l_{m_{l}}$ orbitals are imaginary functions. It is usually more convenient to deal with an equivalent set of real functions, which are linear combinations of these $n l_{m_{l}}$ functions. The shorthand for the real hydrogen orbitals is again $n l$; the added subscript now gives the angular dependency. The complete set of real orbitals for hydrogen through $n=3$ is given in Table 1-1. 
Table 1-1

\section{Important Orbitals for the Hydrogen Atom ${ }^{a}$}

\begin{tabular}{|c|c|c|c|c|c|}
\hline \multicolumn{3}{|c|}{$\begin{array}{c}\text { Orbital quantum } \\
\text { numbers } \\
\end{array}$} & \multirow{2}{*}{$\begin{array}{l}\text { Orbital } \\
\text { desig- } \\
\text { nation }\end{array}$} & \multirow{2}{*}{$\begin{array}{l}\text { Radial function, } \mathrm{b} \\
\qquad R_{n l}(r)\end{array}$} & \multirow{2}{*}{$\begin{array}{c}\text { Angular function, } \\
\phi_{\operatorname{lm}_{l}}\left(\frac{x}{r}, \frac{y}{r}, \frac{z}{r}\right)\end{array}$} \\
\hline$n$ & $l$ & $m_{l}$ & & & \\
\hline 1 & 0 & 0 & is & $2 e^{-r}$ & $\frac{1}{2 \sqrt{\pi}}$ \\
\hline 2 & 0 & 0 & $2 s$ & $\frac{1}{2 \sqrt{2}}(r-2) e^{-r / 2}$ & $\frac{1}{2 \sqrt{\pi}}$ \\
\hline 2 & 1 & $(1)^{d}$ & $2 p_{x}$ & $\frac{1}{2 \sqrt{6}} r e^{-r / 2}$ & $\frac{\sqrt{3}(x / r)}{2 \sqrt{\pi}}$ \\
\hline 2 & 1 & 0 & $2 p_{z}$ & $\frac{1}{2 \sqrt{6}} r e^{-r / 2}$ & $\frac{\sqrt{3}(z / r)}{2 \sqrt{\pi}}$ \\
\hline 2 & 1 & $(-1)^{d}$ & $2 p_{y}$ & $\frac{1}{2 \sqrt{6}} r e^{-r / 2}$ & $\frac{\sqrt{3}(y / r)}{2 \sqrt{\pi}}$ \\
\hline 3 & 0 & 0 & $3 s$ & $\frac{2}{81 \sqrt{3}}\left(27-18 r+2 r^{2}\right) e^{-r / 3}$ & $\frac{1}{2 \sqrt{\pi}}$ \\
\hline 3 & 1 & $(1)^{d}$ & $3 p_{x}$ & $-\frac{4}{81 \sqrt{6}}\left(r^{2}-6 r\right) e^{-r / 3}$ & $\frac{\sqrt{3}(x / r)}{2 \sqrt{\pi}}$ \\
\hline 3 & 1 & 0 & $3 p_{z}$ & $-\frac{4}{81 \sqrt{6}}\left(r^{2}-6 r\right) e^{-r / 3}$ & $\frac{\sqrt{3}(z / r)}{2 \sqrt{\pi}}$ \\
\hline 3 & 1 & $(-1)^{d}$ & $3 p_{y}$ & $-\frac{4}{81 \sqrt{6}}\left(r^{2}-6 r\right) e^{-r / 3}$ & $\frac{\sqrt{3}(y / r)}{2 \sqrt{\pi}}$ \\
\hline 3 & 2 & $(2)^{\mathrm{d}}$ & $3 d_{x^{2}-y^{2}}$ & $\frac{4}{81 \sqrt{30}} r^{2} e^{-r / 3}$ & $\frac{\sqrt{15}\left[\left(x^{2}-y^{2}\right) / r^{2}\right]}{4 \sqrt{\pi}}$ \\
\hline 3 & 2 & $(1)^{d}$ & $3 d_{x z}$ & $\frac{4}{81 \sqrt{30}} r^{2} e^{-r / 3}$ & $\frac{\sqrt{30}\left(x z / r^{2}\right)}{2 \sqrt{2 \pi}}$ \\
\hline 3 & 2 & 0 & $3 d_{z^{2}}$ & $\frac{4}{81 \sqrt{30}} r^{2} e^{-r / 3}$ & $\frac{\sqrt{5}\left[\left(3 z^{2}-r^{2}\right) / r^{2}\right]}{4 \sqrt{\pi}}$ \\
\hline 3 & 2 & $(-1)^{\mathrm{d}}$ & $3 d_{y z}$ & $\frac{4}{81 \sqrt{30}} r^{2} e^{-r / 3}$ & $\frac{\sqrt{30}\left(y z / r^{2}\right)}{2 \sqrt{2 \pi}}$ \\
\hline 3 & 2 & $(-2)^{\mathrm{d}}$ & $3 d_{x y}$ & $\frac{4}{81 \sqrt{30}} r^{2} e^{-r / 3}$ & $\frac{\sqrt{15}\left(x y / \gamma^{2}\right)}{2 \sqrt{\pi}}$ \\
\hline
\end{tabular}

${ }^{a}$ Both the radial and the angular functions are normalized to one; $r$ is in atomic units (that is, in units of $a_{0}$; see problem 1-1).

$\mathrm{b}_{\text {To }}$ convert to a general radial function for a one-electron atom with any nuclear charge $Z$, replace $r$ by $Z r$ and multiply each function by $(Z)^{3 / 2}$.

${ }^{c}$ Often expressed in the spherical coordinates $\theta$ and $\phi$ by replacing $x$ with $r \sin \theta \times$ $\cos \phi, y$ with $r \sin \theta \sin \phi$, and $z$ with $r \cos \theta$.

dit is not correct to assign $m_{l}$ values to the real functions $x, y, x^{2}-y^{2}, x z, y z$, and $x y$. 
It is common practice to make drawings of the hydrogen orbitals, outlining the region within which there is a large probability for finding the electron. Remember that the electronic density in an orbital is related to the square of the absolute value of the wave function. Keep this in mind when you encounter dual-purpose drawings of the boundary surfaces of orbitals, which outline 90 per cent, say, of $|\psi|^{2}$, and also indicate the + and - signs on the lobes given by the angular part of $\psi$. The boundary-surface pictures are very useful and should be memorized. The boundary surfaces for $s, p, d$, and $f$ orbitals are given in Figs. 1-3, 1-4, 1-5, and 1-6, along with radial-distribution graphs for the different orbitals.

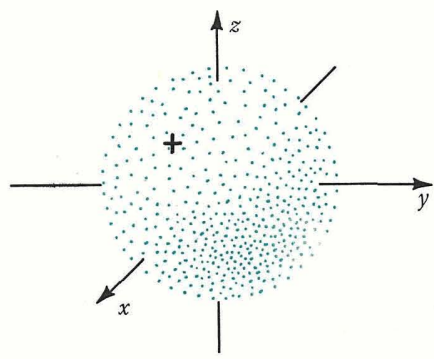

(a) $s$ orbital

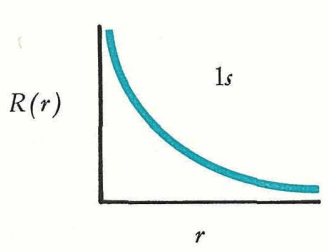

(b)
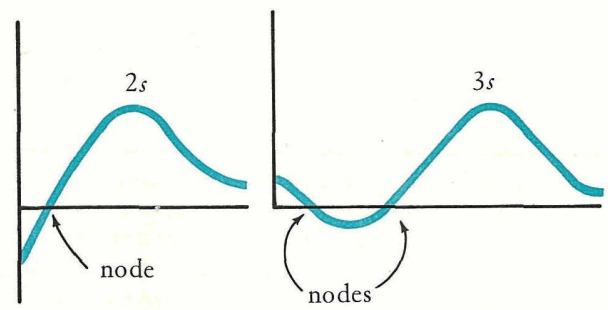

Figure 1-3 (a) Boundary surface of an $s$ orbital. (b) Plots of the radial function $R(r)$ vs. $r$ for $1 s, 2 s$, and $3 s$ orbitals. The $2 s$ radial function changes sign as $r$ increases. Thus there is a point where $\boldsymbol{R}(\boldsymbol{r})=0$ for the $2 s$ radial function. Such a zero point is called a node. The $3 s$ radial function has two nodes. 


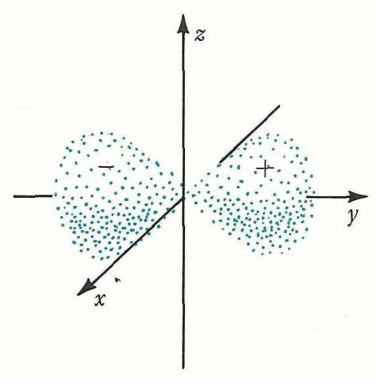

$$
p_{y} \text { orbital }
$$

(a)

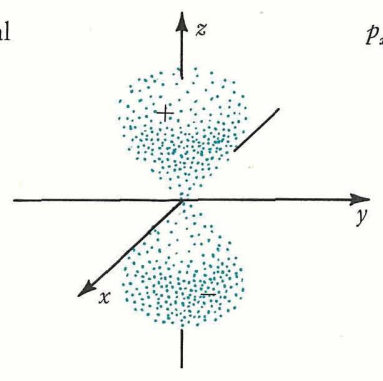

$$
\text { pzorbital }
$$

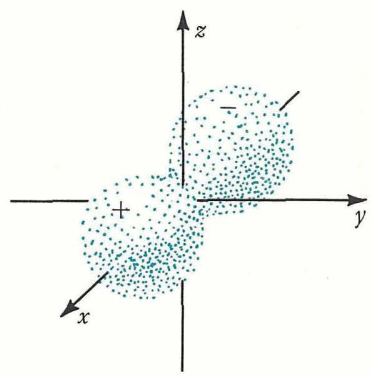

$p_{x}$ orbital

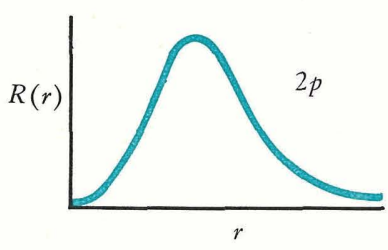

(b)

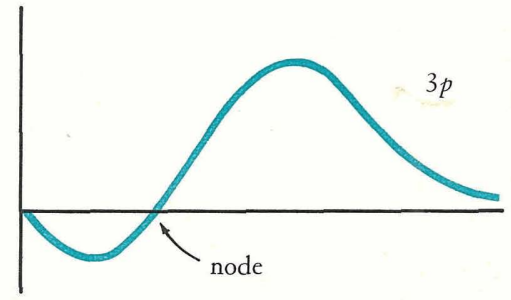
Figure 1-4 (a) Boundary surfaces of the $p$ orbitals. (b) Plots
of the radial function $R(r)$ vs. $r$ for $2 p$ and $3 p$ orbitals. The $3 p$ orbital has one node, as indicated.

\section{1-13 ELECTRON SPIN}

The three quantum numbers $n, l$, and $m_{l}$ are all associated with the movement of the electron around the nucleus of the hydrogen atom. In order to explain certain precise spectral observations, Goudsmit 

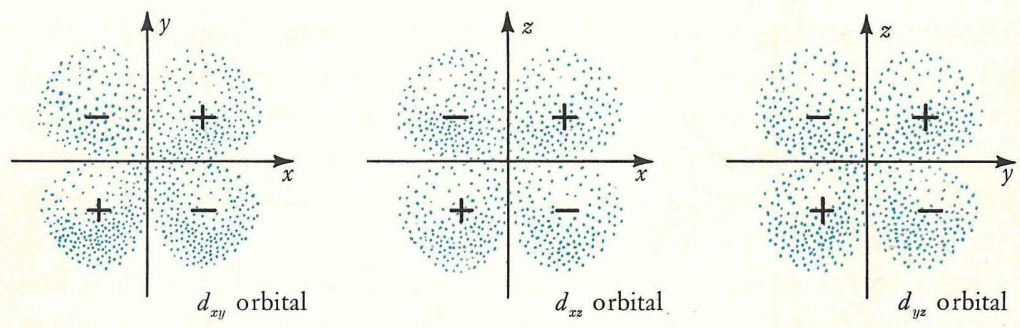

(a)
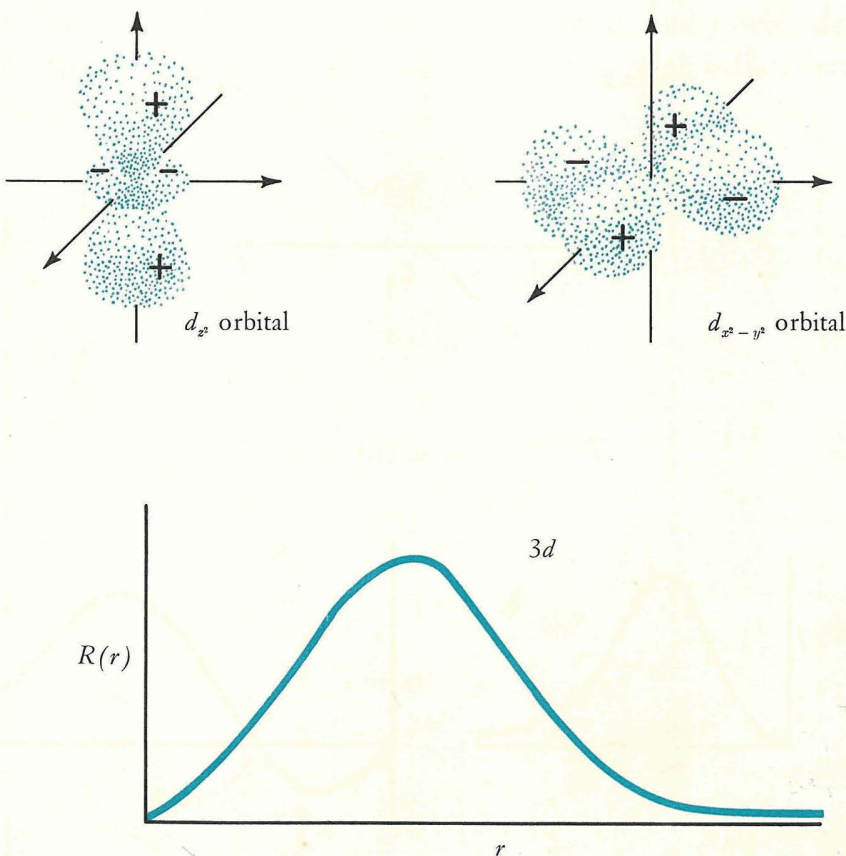

(b)

Figure 1-5 (a) Boundary surfaces of the $d$ orbitals. (b) Plot of $\boldsymbol{R}(r)$ vs. $\boldsymbol{r}$ for a $3 d$ orbital. 

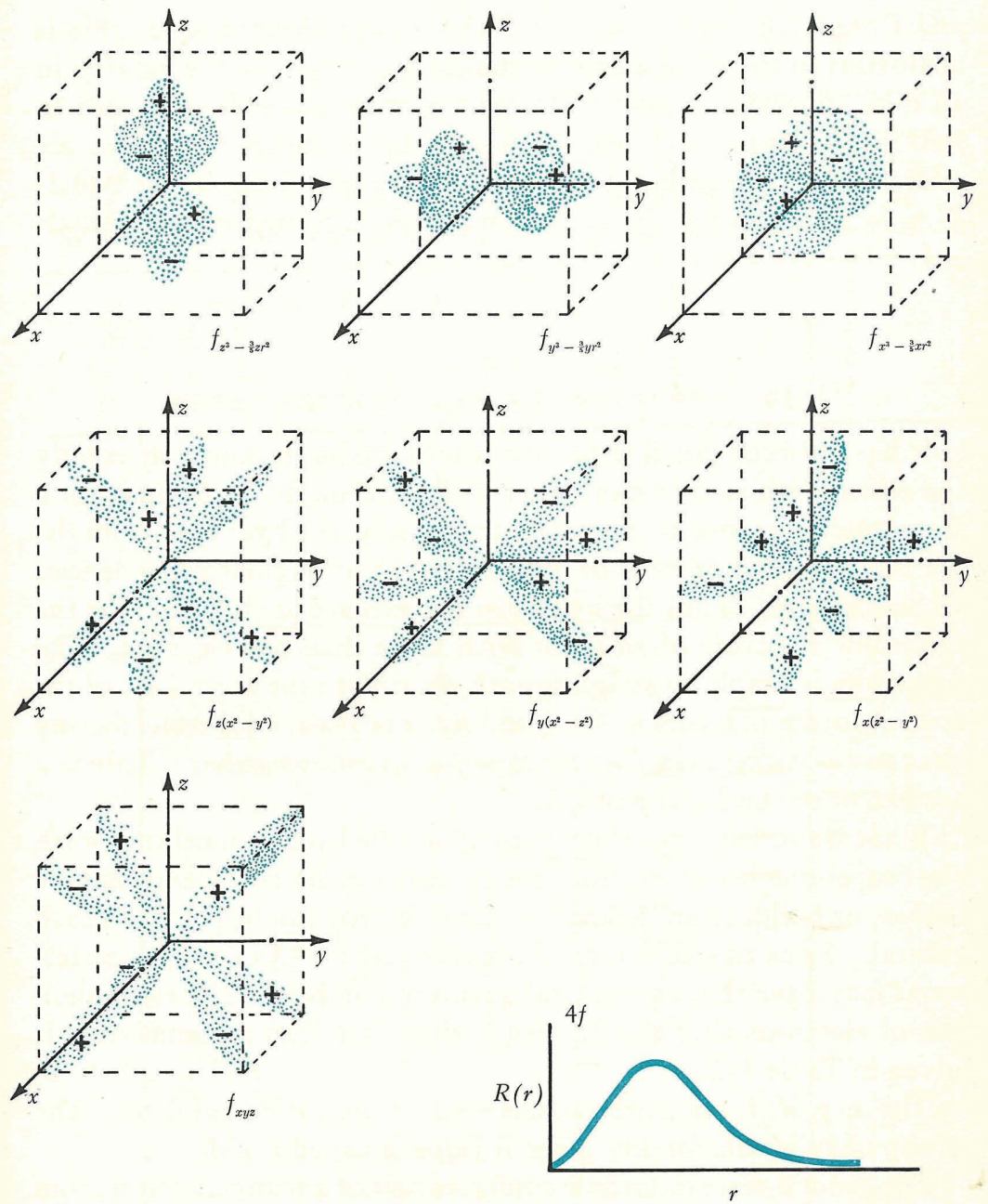

(a)

(b)

Figure 1-6 (a) Boundary surfaces of the $f$ orbitals. (b) Plot of $R(r)$ vs. $r$ for a $4 f$ orbital. 
and Uhlenbeck (1925) introduced the idea of electron spin (this is analogous to the earth spinning about its own axis while moving in an orbit around the sun). The spin of an electron is quantized in half-integer units, and two more quantum numbers, $s$ and $m_{s}$, are added to our collection: $s$ is called the spin quantum number and equals $\frac{1}{2} ; m_{s}$ is related to $s$ in the same way that $m_{l}$ is related to $l$ and equals $\pm \frac{1}{2}$.

\section{1-14 THE THEORY OF MANY-ELECTRON ATOMS}

It has not been possible to solve the Schrödinger equation exactly for atoms with two or more electrons. Although the orbitals for a many-electron atom are not quite the same as the hydrogen orbitals, we do expect the number of orbitals and their angular dependencies to be the same. Thus the hydrogen orbitals are used to describe the electronic structure of an atom with more than one electron. The procedure is simply to assign to each electron in the atom a set of the four quantum numbers $n, l, m_{l}$, and $m_{s}$ ( $s$ is always $\frac{1}{2}$ ), remembering that no two electrons can bave the same four quantum numbers. This is a statement of the Pauli principle.

What we actually do, then, is to fill up the hydrogen orbitals with the proper number of electrons for the atom under consideration (the aufbau, or building up, principle). One electron can be placed in each orbital. Since an electron can have $m_{s}$ equal to $+\frac{1}{2}$ or $-\frac{1}{2}$, two electrons may have the same orbital quantum numbers. The total number of electrons that the different orbital sets can accommodate is given in Table 1-2.

The $s, p, d, f$, etc., orbital sets usually are called subsbells. The group of subshells for any given $n$ value is called a shell.

The ground-state electronic configuration of a many-electron atom is of greatest interest. In order to determine the ground state of a many-electron atom the orbital sets are filled up in order of increasing energy until all the electrons have been accommodated. We know from experimental observations that the order of increasing energy of the orbital sets in many-electron neutral atoms is $1 s, 2 s, 2 p, 3 s, 3 p$, $4 s, 3 d, 4 p, 5 s, 4 d, 5 p, 6 s, 4 f, 5 d, 6 p, 7 s, 5 f \sim 6 d$. A diagram showing the energies of the orbitals in neutral atoms is given in Fig. 1-7. 
Table $1-2$

The $s, p, d$, and $f$ Orbltal Sets

\begin{tabular}{cccc}
$\begin{array}{c}\text { Type } \\
\text { of } \\
\text { orbital }\end{array}$ & $\begin{array}{c}\text { Orbital } \\
\text { quantum numbers }\end{array}$ & $\begin{array}{c}\text { Total } \\
\text { orbitals } \\
\text { in set }\end{array}$ & $\begin{array}{c}\text { Total mimber } \\
\text { of electrons } \\
\text { that can be } \\
\text { accommodated }\end{array}$ \\
\hline$s$ & $l=0 ; m_{l}=0$ & 1 & 2 \\
$p$ & $l=1 ; m_{l}=1,0,-1$ & 3 & 6 \\
$d$ & $l=2 ; m_{l}=2,1,0,-1,-2$ & 5 & 10 \\
$f$ & $l=3 ; m_{l}=3,2,1,0,-1,-2,-3$ & 7 & 14 \\
\hline
\end{tabular}

high energy

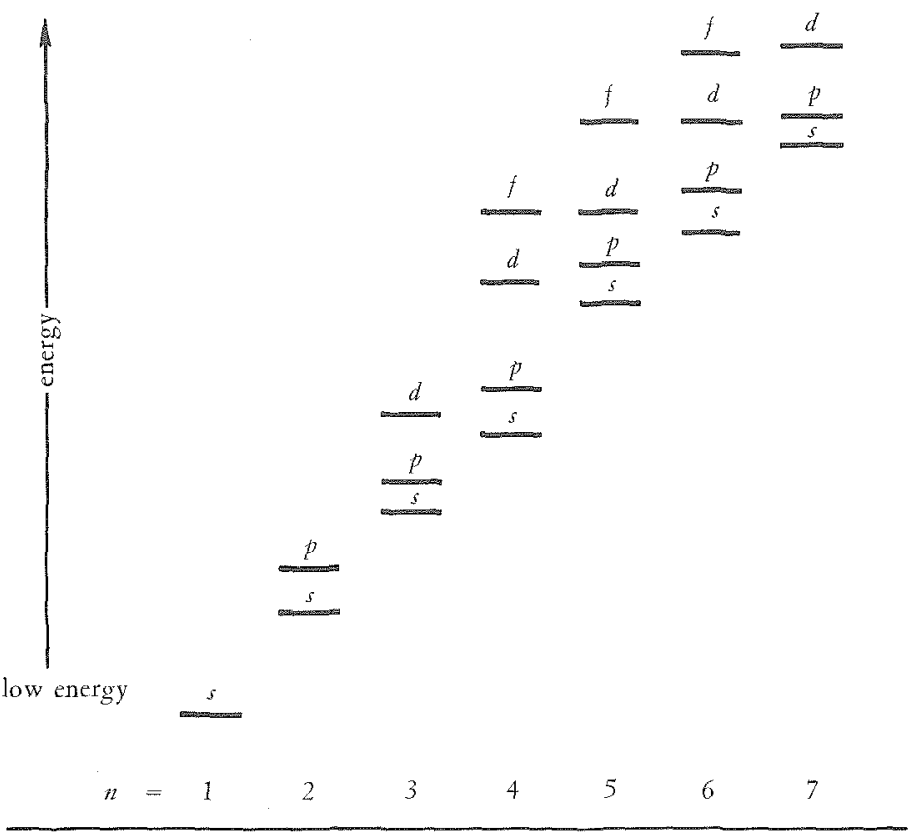

Figure 1-7 Relative energies of the orbitals in neutral atoms. 


\section{1-15 RUSSELL-SAUNDERS TERMS}

It is convenient to classify an atomic state in terms of total orbital angular momentum $L$ and total spin $S$ (capital letters always are used for systems of electrons; small letters are reserved for individual electrons). This Russell-Saunders $L S M_{L} M_{S}$ scheme will now be described in detail.

For a system of $n$ electrons, we define

$$
\begin{aligned}
& M_{L}=m_{l_{1}}+m_{l_{2}}+m_{l_{3}}+\cdots+m_{l_{n}} \\
& M_{S}=m_{s_{1}}+m_{s_{2}}+m_{s_{3}}+\cdots+m_{s_{n}}
\end{aligned}
$$

We also have these relationships between $L$ and $M_{L}, S$ and $M_{S}$ :

$$
\begin{aligned}
& M_{L}=L, L-1, L-2, \cdots,-L \\
& M_{S}=S, S-1, S-2, \cdots,-S
\end{aligned}
$$

Let us take the lithium atom as an illustrative example. The atomic number (the number of protons or electrons in the neutral atom) of lithium is 3 . Therefore the orbital electronic configuration of the ground state is $(1 s)^{2}(2 s)^{1}$. The ground-state $L S M_{L} M_{S}$ term is found as follows:

1. Find the possible values of $M_{L}$.

$$
\begin{aligned}
& M_{L}=m_{l_{1}}+m_{l_{2}}+m_{l_{3}} \\
& m_{l_{1}}=m_{l_{2}}=m_{l_{3}}=0 \quad \text { (all are } s \text { electrons) } \\
& M_{L}=0
\end{aligned}
$$

2. Find the possible values of $L$.

$$
\begin{aligned}
& M_{L}=0 \\
& L=0
\end{aligned}
$$

3. Find the possible values of $M_{S}$.

$$
\begin{aligned}
& M_{S}=m_{s_{1}}+m_{s_{2}}+m_{s_{3}} \\
& m_{s_{1}}=+\frac{1}{2}, \quad m_{s_{2}}=-\frac{1}{2}, \quad m_{s_{3}}= \pm \frac{1}{2} \\
& M_{\mathrm{S}}=+\frac{1}{2} \quad \text { or } \quad-\frac{1}{2}
\end{aligned}
$$

4. Find the possible values of $S$.

$$
\begin{aligned}
& M_{S}=+\frac{1}{2},-\frac{1}{2} \\
& S=\frac{1}{2}
\end{aligned}
$$


A Russell-Saunders term is written in the shorthand notation ${ }^{2 S+1} L$. The superscript $2 S+1$ gives the number of different $M_{S}$ values of any state, often referred to as the spin multiplicity. As in the single-electron-orbital shorthand, letters are used for $L . \quad(L=0$ is $S ; L=1$ is $P ; L=2$ is $D ; L=3$ is $F$; etc.) For the lithium atom, the groundstate term has $L=0$ and $S=\frac{1}{2}$, designated ${ }^{2} S$. An excited electronic configuration for lithium would be $(1 s)^{2}(2 p)^{1}$. For this configuration, we find $M_{L}=1,0,-1(L=1)$ and $M_{S}= \pm \frac{1}{2}\left(S=\frac{1}{2}\right)$. Therefore the term designation of this particular excited state is ${ }^{2} P$.

Admittedly the lithium atom is a very simple case. To find the term designations of the ground state and excited states for more complicated electronic structures, it helps to construct a chart of the possible $M_{L}$ and $M_{S}$ values. This more general procedure may be illustrated with the carbon atom. The carbon atom has six electrons. Thus the orbital configuration of the ground state must be $(1 s)^{2}(2 s)^{2}(2 p)^{2}$. It remains for us to find the correct ground-state term.

First a chart is drawn as shown in Fig. 1-8a, placing the possible values of $M_{L}$ in the left-hand column and the possible values of $M_{S}$ in the top row. We need consider only the electrons in incompletely filled subshells. Filled shells or subshells may be ignored in constructing such a chart since they always give a contribution $M_{L}=$ $O(L=0)$ and $M_{S}=O(S=0)$. (Convince yourself of this before proceeding.) For carbon the configuration $(2 p)^{2}$ is important. Each of the two $p$ electrons has $l=1$ and can therefore have $m_{l}=+1,0$, or -1 . Thus the values possible for $M_{L}$ range from +2 to -2 .

Each of the two $p$ electrons can have $m_{s}=+\frac{1}{2}$ or $-\frac{1}{2}$. Thus the values possible for $M_{S}$ are 1,0 , and -1 .

The next step is to write down all the allowable combinations (called microstates) of $m_{l}$ and $m_{s}$ values for the two $p$ electrons and to place these microstates in their proper $M_{L}, M_{S}$ boxes. The general form for these microstates is

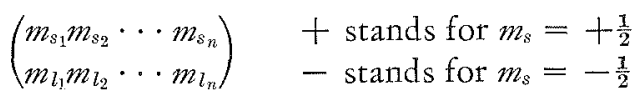

The microstate that fits in the $M_{L}=2, M_{S}=1$ box is $(1,1)$. However, since for both the $2 p$ electrons under consideration $n=2$ and 


\begin{tabular}{|c|c|c|c|}
\hline$M_{L} M_{S}$ & 1 & 0 & -1 \\
\hline 2 & $\begin{array}{l}(1,+ \\
\text { Pauli }\end{array}$ & $\stackrel{+}{(1, \overline{1})}$ & \\
\hline 1 & $\stackrel{+}{+}+\stackrel{+}{1})$ & $(\stackrel{+}{+}, \overline{0}) \quad\left({ }_{1}^{+}, 0\right)$ & $(\overline{1}, \overline{0})$ \\
\hline 0 & $(\stackrel{+}{1},-+\dot{1})$ & $\begin{array}{rr}\stackrel{+}{1},-\overline{1}) & (\overline{1},-\stackrel{+}{1}) \\
& (+-\overline{0} \\
& (0,0)\end{array}$ & $(\overline{1},-\overline{1})$ \\
\hline-1 & $(-\stackrel{++}{-1,0})$ & $(-\stackrel{+}{+}, \overline{0}) \quad\left(-\overline{1}^{-},{ }^{+}\right)$ & $(-\overline{1}, \overline{0})$ \\
\hline-2 & & $\left(-{ }_{1}^{+},-\overline{1}\right)$ & \\
\hline
\end{tabular}

(a)

\begin{tabular}{|c|c|c|c|c|}
\hline$M_{L_{1}} M_{\mathrm{S}}$ & 1 & \multicolumn{2}{|c|}{0} & -1 \\
\hline 2 & & \multicolumn{2}{|c|}{$\stackrel{+-}{(1,1)}$} & \\
\hline 1 & $\left(\begin{array}{l}+ \\
1,0\end{array}\right.$ & $(+1,0)$ & $(\overrightarrow{+},++)$ & $(\overline{1}, \overline{0})$ \\
\hline 0 & $(1,-1)$ & $(1,-1)$ & $\begin{array}{c}(\overline{1},-1) \\
+(-\overline{+}) \\
(0,0)\end{array}$ & $(1,-1)$ \\
\hline-1 & $(-1,0)$ & $(-1,0)$ & $(-\overline{1}, \stackrel{+}{0})$ & $(-\overline{1}, \overline{0})$ \\
\hline-2 & & $(-\stackrel{+}{1,-}-$ & & \\
\hline
\end{tabular}

(b)

Figure 1-8 (a) $M_{L}, M_{S}$ microstate chart for the $(2 p)^{2}$ orbital configuration. (b) $M_{L}, M_{S}$ microstate chart for the $(2 p)^{2}$ orbital configuration; the ${ }^{3} P$ term has been eliminated by crossing out the six microstates in the $M_{S}=1$ and $M_{S}=-1$ columns and, randomly; three microstates with $M_{L}$ equal to 1,0 , and -1 in the $M_{S}=0$ column.

$l=1$, this microstate is not allowable according to the Pauli principle and is crossed out in Fig. 1-8a.

Proceeding to the $M_{L}=1, M_{S}=1$ box, the microstate ( $\left.1, \pm+0\right)$ fits and is allowable. The two electrons may both have $m_{l}=+1$ 
and therefore $M_{L}=2$ if their $m_{s}$ values differ. Thus the microstate ( $\dot{1}, \overline{1})$ is allowable and fits in the $M_{L}=2, M_{S}=0$ box. This procedure is followed until the chart is completed.

From the completed chart the ${ }^{2 S+1} L$ terms may be written down. Start at top left on the chart. There is a microstate with $M_{L}=1$, $M_{S}=1$. This microstate may be considered the parent of a state that has $L=1, S=1$, or ${ }^{3} P$. From Eqs. (1-40) and (1-41), we see that a term with $L=1$ and $S=1$ has all possible combinations of $M_{L}=1,0,-1$ and $M_{S}=1,0,-1$. Therefore, a ${ }^{3} P$ state must have, in addition to the $M_{L}=1, M_{S}=1$ microstate, microstates with $M_{L}=0, M_{S}=1 ; M_{L}=-1, M_{S}=1 ; M_{L}=1, M_{S}=0 ; M_{L}=0$, $M_{S}=0 ; M_{L}=-1, M_{S}=0 ; M_{L}=1, M_{S}=-1 ; M_{L}=0, M_{S}=$ $-1 ; M_{L}=-1, M_{S}=-1$. Thus a total of nine microstates are accounted for by the ${ }^{3} P$ term. Subtracting these nine microstates from the chart, we are left with a new puzzle, as shown in Fig. 1-8b.

Moving across the top row, there is a microstate with $M_{L}=2$, $M_{S}=0$, which may be considered the parent of a state that has $L=2, S=0$, or ${ }^{1} D$. The ${ }^{1} D$ state also must have microstates $M_{L}=$ $1, M_{S}=0 ; M_{L}=0, M_{S}=0 ; M_{L}=-1, M_{S}=0 ; M_{L}=-2, M_{S}=$ 0 . Subtracting these five combinations of the ${ }^{1} D$ state, we are left with a single microstate in the $M_{L}=0, M_{S}=0$ box. This microstate indicates that there is a term having $L=0, S=0$, or ${ }^{1} S$.

We now have the three terms, ${ }^{3} P,{ }^{1} D$, and ${ }^{1} S$, which account for all the allowable microstates arising from the $(2 p)^{2}$ electronic configuration. The ground-state term always has maximum spin multiplicity. This is Hund's first rule. Therefore, for the carbon atom, the ${ }^{3} P$ term is the ground state.

The ${ }^{1} D$ and ${ }^{1} S$ terms are excited states having the $(2 p)^{2}$ orbital electronic configuration. Hund's second rule says that, when comparing two states of the same spin multiplicity, the state with the higher value of $L$ is usually more stable. This is the case with the ${ }^{1} D$ and ${ }^{1} S$ terms for the carbon atom, since the ${ }^{1} D$ state is more stable than the ${ }^{1} S$ state.

\section{PROBLEMS}

1-6. Work out the ground-state and excited-state terms for the most stable orbital electronic configuration of the titanium atom. Solution. The atomic number of titanium is 22 . Thus the most 
stable orbital electronic configuration is $(1 s)^{2}(2 s)^{2}(2 p)^{6}(3 s)^{2}(3 p)^{6}$ $(4 s)^{2}(3 d)^{2}$. The only incompletely filled subshell is $3 d$.

Examine Table $1-3$, the $M_{L}, M_{S}$ chart for the $(3 d)^{2}$ configuration. The $(\overrightarrow{2}, \overrightarrow{+})$ microstate is the parent of a ${ }^{3} F$ term. The ${ }^{3} F$ term

Table $1-3$

Values of $M_{L}, M_{S}$ for $(3 d)^{2}$ Configuration

\begin{tabular}{|c|c|c|c|}
\hline \multirow[b]{2}{*}{$M_{L}$} & \multicolumn{3}{|c|}{$M_{S}$} \\
\hline & 1 & 0 & -1 \\
\hline 4 & & $(+\overline{2}, \overline{2})$ & \\
\hline 3 & $\begin{array}{c}++ \\
(2,1)\end{array}$ & $(+\overline{2}, \overline{1})(\overline{2},++$ & $(\overline{2}, \overline{1})$ \\
\hline 2 & $\begin{array}{c}++ \\
(2,0)\end{array}$ & $(+\overline{0})(\overline{0})++(+\overline{0})(\overline{1})$ & $(\overline{2}, \overline{0})$ \\
\hline 1 & $(\stackrel{++}{+}+\stackrel{+}{1}, \stackrel{+}{0})(2,-1)$ & $\begin{array}{l}(+\overline{1}, \overline{0})(\overline{1},+) \\
+(-\overline{0}) \\
(2,-\overline{1})(\overline{2},-\dot{1})\end{array}$ & $(\overline{1}, \overline{0})(\overline{2},-\overline{1})$ \\
\hline 0 & 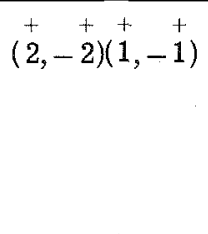 & $\begin{array}{l}+(-\overline{2})(\overline{2},+2) \\
(2,-2) \\
+(\overline{1},-\overline{1}) \\
(\overline{1},+++\overline{1})(\overline{0}, \overline{0})\end{array}$ & $(\overline{2},-\overline{2})(\overline{1},-\overline{1})$ \\
\hline-1 & $\left(\begin{array}{c}+++ \\
(-1,0)(1,\end{array}\right.$ & $\begin{array}{c}+\overline{-} \overline{0})(-\overline{1}, 0) \\
+(-\overline{1})(-\overline{2}, 1) \\
(-2,1)\end{array}$ & $(-\overline{1}, \overline{0})(\overline{1},-\overline{2})$ \\
\hline-2 & $\left(\begin{array}{r}++ \\
(-2,0)\end{array}\right.$ & $(-2, \overline{0})(-\overline{2}, 0)(-1,-\overline{1})$ & $(-\overline{2}, \overline{0})$ \\
\hline-3 & $\left(\begin{array}{c}+ \\
(-2,-1 \\
-1\end{array}\right)$ & $\left(-{ }^{+},-\overline{1}\right)(-\overline{2},-1)$ & $(-\overline{2},-\overline{1})$ \\
\hline-4 & & $(-\stackrel{+}{2},-\overline{2})$ & \\
\hline
\end{tabular}


accounts for 21 microstates. Starting at the $M_{L}=1, M_{B}=1$ box, there are two microstates. Thus there also must be a ${ }^{3} P$ term. The $(2, \overline{2})$ microstate is the parent of a ${ }^{1} G$ term. The terms ${ }^{1} D$ and ${ }^{1} S$ account for the remaining microstates in the $M_{S}=0$ column.

The ground-state term has maximum spin multiplicity and must be either ${ }^{3} F$ or ${ }^{3} P$. The ${ }^{3} F$ state has the higher angular momentum $(L=3)$ and is predicted to be the ground state. The ${ }^{3} F$ term is the experimentally observed ground state for the titanium atom. The ${ }^{3} P$ state is the first excited state, with the ${ }^{1} G,{ }^{1} D$, and ${ }^{1} S$ states more unstable.

1-7. Using Table 1-4, work out the terms arising from the orbital electronic configuration $(3 d)^{1}(4 d)^{1}$, and designate the most stable state.

Solution. The $(3 d)^{1}(4 d)^{1}$ problem is slightly different from the $(3 d)^{2}$ probletn. Both electrons are $d$ electrons with $l=2$, but one has $n=3$ and one has $n=4$. Thus, for example, the $(2,+2)$ microstate does not violate the Pauli principle, since the $n$ quantum numbers differ. The bookkeeping is simplified by adding a subscript 4 to the $m_{l}$ value for the $4 d$ electron.

The terms deduced from the chart for the $(3 d)^{1}(4 d)^{1}$ configuration are ${ }^{3} G,{ }^{3} F,{ }^{3} D,{ }^{3} P,{ }^{3} S,{ }^{1} G,{ }^{1} F,{ }^{1} D,{ }^{1} P$, and ${ }^{1} S$. Following the spinmultiplicity and angular-momentum rules, the ${ }^{3} G$ state should be most stable.

\section{1-16 IONIZATION POTENTIALS}

The ionization potential (abbreviated IP) of an atom is the minimum energy required to completely remove an electron from the atom. This process may be written

$$
\text { atom }+\mathrm{IP} \text { (energy) } \rightarrow \text { unipositive ion }+ \text { electron }
$$

Further ionizations are possible for all atoms but hydrogen. In general, the ionization energy required to detach the first electron is called $\mathrm{IP}_{1}$, and subsequent ionizations require $\mathrm{IP}_{2}, \mathrm{IP}_{3}, \mathrm{IP}_{4}$, etc. Quite obviously, for any atom there are exactly as many IP's as electrons.

The first ionization potentials for most of the atoms are given in Table 1-5. For any atom, the $\mathrm{IP}_{1}$ is always the smallest IP. This is understandable since removal of a negatively charged particle 
Table $1-4$

Value of $M_{\mathcal{L}}, M_{S}$ Lo: $(3 d)^{2}(4 d)^{2}$ Configuration

\begin{tabular}{|c|c|c|c|}
\hline \multirow[b]{2}{*}{$M_{L}$} & \multicolumn{3}{|c|}{$M_{S}$} \\
\hline & 1 & 0 & -1 \\
\hline 4 & $\begin{array}{c}++ \\
\left(2,2_{4}\right)\end{array}$ & $\left(2, \overline{2}_{4}\right)\left(2,2_{4}\right)$ & $\left(\overline{2}, \overline{2_{4}}\right)$ \\
\hline 3 & $\left(\begin{array}{l}++ \\
\left(2,1_{4}\right.\end{array}\right)\left(\begin{array}{l}+ \\
2_{4}\end{array},++1\right)$ & $\begin{array}{l}\left.+\overline{2}_{2}\right)\left(\overline{1}_{4}\right)\left(\overline{1}_{4}\right) \\
+\left(-\overline{1}_{4}+\right. \\
\left(2_{4}, \overline{1}_{1}\right)\left(\overline{2}_{4},{ }_{1}\right)\end{array}$ & $\left(\overline{2}_{3}, \overline{1}_{4}\right)\left(\overline{2}_{4}, \overline{1}\right)$ \\
\hline 2 & $\begin{array}{l}++++ \\
\left(2,0_{4}\right)\left(2_{4}, 0\right) \\
++ \\
\left(1,1_{4}\right)\end{array}$ & 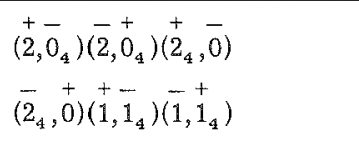 & $\begin{array}{l}\left(\overline{2}, \overline{0}_{4}\right)\left(\overline{2}_{4}, \overline{0}\right) \\
\left(\overline{1}, \overline{1}_{4}\right)\end{array}$ \\
\hline 1 & 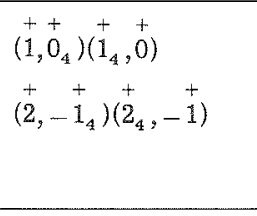 & 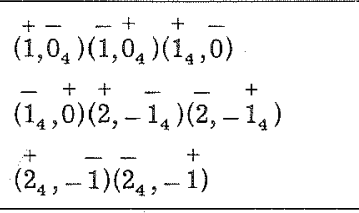 & $\begin{array}{l}\left(\overline{1}, \overline{0}_{4}\right)\left(\overline{1}_{4}, \overline{0}\right) \\
\left(\overline{2},-\overline{1}_{4}\right)\left(\overline{2}_{4},-\overline{1}\right)\end{array}$ \\
\hline 0 & $\begin{array}{l}\left.++\underset{+}{+}+\underset{1}{1},-1_{4}\right)\left(1_{4},-1\right) \\
++++ \\
\left(2,-2_{4}\right)\left(2_{4},-2\right) \\
++ \\
\left(0,0_{4}\right)\end{array}$ & 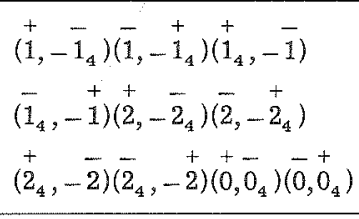 & $\begin{array}{l}\left(\overline{1},-\overline{1}_{4}\right)\left(\overline{1}_{4},-\overline{1}\right) \\
\left(\overline{2},-\overline{2}_{4}\right)\left(\overline{2}_{4},-\overline{2}\right) \\
\left(\overline{0}, \overline{0}_{4}\right)\end{array}$ \\
\hline-1 & 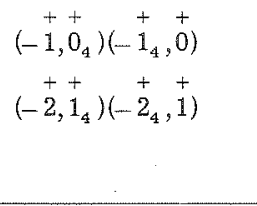 & 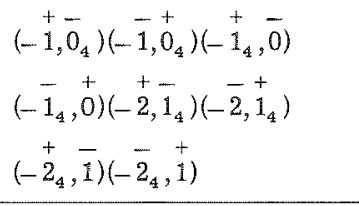 & $\begin{array}{l}\left(-\overline{1}_{1}, \overline{0}_{4}\right)\left(-\overline{1}_{4}, \overline{0}\right) \\
\left(-\overline{2}_{1}, \overline{1}_{4}\right)\left(-\overline{2}_{4}, \overline{1}\right)\end{array}$ \\
\hline-2 & $\begin{array}{l}++++++ \\
\left(-2,0_{4}\right)\left(-2_{4}, 0\right) \\
++ \\
\left(-1,-1_{4}\right)\end{array}$ & 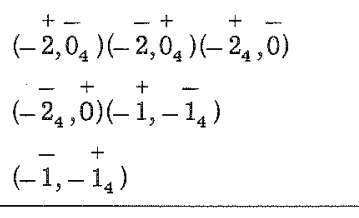 & $\begin{array}{l}\left(-\overline{2}, \overline{0}_{4}\right)\left(-\overline{2}_{4}, \overline{0}\right) \\
\left(-\overline{1},-\overline{1}_{4}\right)\end{array}$ \\
\hline-3 & 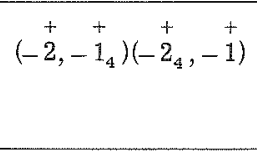 & $\begin{array}{c}++ \\
\left(-\overline{2}_{2},-\overline{1}_{4}\right)\left(-\overline{2}_{2},-1_{4}\right) \\
++ \\
\left(-2_{4},-\overline{1}\right)\left(-\overline{2}_{4},-1\right)\end{array}$ & $\left(-\overline{2},-\overline{1}_{4}\right)\left(-\overline{2}_{4},-\overline{1}\right)$ \\
\hline-4 & $\left(\begin{array}{r}+ \\
-2\end{array}-2_{4}^{+}\right)$ & $\left(\stackrel{+}{+},-\overline{2}_{4}\right)\left(-\overline{2}_{2},-{ }_{2}^{+}\right)$ & $\left(-\overline{2},-\overline{2}_{4}\right)$ \\
\hline
\end{tabular}


Table $1-5$

The Electronic Configurations and Ionization Potentials of Atoms

\begin{tabular}{|c|c|c|c|c|}
\hline$Z$ & Atom (A) & $\begin{array}{c}\text { Orbital electronic } \\
\text { configuration }\end{array}$ & $\begin{array}{c}\text { Ground } \\
\text { state } \\
\text { term }\end{array}$ & $I P_{1}, e V^{a}$ \\
\hline 1 & $\mathrm{H}$ & $1 s$ & ${ }^{2} S$ & 13.595 \\
\hline 2 & $\mathrm{He}$ & $1 s^{2}$ & ${ }^{1} S$ & 24.580 \\
\hline 3 & Li & {$[\mathrm{He}] 2 \mathrm{~s}$} & ${ }^{2} S$ & 5.390 \\
\hline 4 & $\mathrm{Be}$ & {$[\mathrm{He}] 2 s^{2}$} & ${ }^{1} S$ & 9.320 \\
\hline 5 & $\mathrm{~B}$ & {$[\mathrm{He}] 2 s^{2} 2 p$} & ${ }^{2} P$ & 8.296 \\
\hline 6 & $\mathrm{C}$ & {$[\mathrm{He}] 2 s^{2} 2 p^{2}$} & ${ }^{3} P$ & 11.264 \\
\hline 7 & $\mathrm{~N}$ & {$[\mathrm{He}] 2 s^{2} 2 p^{3}$} & ${ }^{4} S$ & 14.54 \\
\hline 8 & 0 & {$[\mathrm{He}] 2 s^{2} 2 p^{4}$} & ${ }^{3} P$ & 13.614 \\
\hline 9 & $\mathrm{~F}$ & {$[\mathrm{He}] 2 s^{2} 2 p^{5}$} & ${ }^{2} P$ & 17.42 \\
\hline 10 & $\mathrm{Ne}$ & {$[\mathrm{He}] 2 s^{2} 2 p^{6}$} & ${ }^{I} S$ & 21.559 \\
\hline 11 & $\mathrm{Na}$ & {$[\mathrm{Ne}] 3 \mathrm{~s}$} & ${ }^{2} S$ & 5.138 \\
\hline 12 & $\mathrm{Mg}$ & {$[\mathrm{Ne}] 3 s^{2}$} & ${ }^{1} S$ & 7.644 \\
\hline 13 & $\mathrm{Al}$ & {$[\mathrm{Ne}] 3 s^{2} 3 p$} & ${ }^{2} P$ & 5.984 \\
\hline 14 & $\mathrm{Si}$ & {$[\mathrm{Ne}] 3 s^{2} 3 p^{2}$} & ${ }^{3} P$ & 8.149 \\
\hline 15 & $\mathrm{P}$ & {$[\mathrm{Ne}] 3 s^{2} 3 p^{3}$} & ${ }^{4} S$ & 11.0 \\
\hline 16 & $\mathrm{~S}$ & {$[\mathrm{Ne}] 3 s^{2} 3 p^{4}$} & ${ }^{3} P$ & 10.357 \\
\hline 17 & $\mathrm{Cl}$ & {$[\mathrm{Ne}] 3 s^{2} 3 p^{5}$} & ${ }^{2} P$ & 13.01 \\
\hline 18 & $\mathrm{Ar}$ & {$[\mathrm{Ne}] 3 s^{2} 3 p^{6}$} & ${ }^{1} S$ & 15.755 \\
\hline 19 & $K$ & {$[\mathrm{Ar}] 4 \mathrm{~s}$} & ${ }^{2} S$ & 4.339 \\
\hline 20 & $\mathrm{Ca}$ & {$[\mathrm{Ar}] 4 \mathrm{~s}^{2}$} & ${ }^{1} S$ & 6.111 \\
\hline 21 & $\mathrm{Sc}$ & {$[\mathrm{Ar}] 4 s^{2} 3 d$} & ${ }^{2} D$ & 6.56 \\
\hline 22 & $\mathrm{Ti}$ & {$[\mathrm{Ar}] 4 s^{2} 3 d^{2}$} & ${ }^{3} E$ & 6.83 \\
\hline 23 & V & {$[\mathrm{Ar}] 4 s^{2} 3 d^{3}$} & ${ }^{4} F$ & 6.74 \\
\hline 24 & $\mathrm{Cr}$ & {$[\mathrm{Ar}] 4 s 3 d^{5}$} & ${ }^{7} S$ & 6.763 \\
\hline 25 & $\mathrm{Mn}$ & {$[\mathrm{Ar}] 4 s^{2} 3 d^{5}$} & ${ }^{6} S$ & 7.432 \\
\hline 26 & $\mathrm{Fe}$ & {$[\mathrm{Ar}] 4 s^{2} 3 d^{6}$} & ${ }^{5} D$ & 7.90 \\
\hline 27 & Co & {$[\mathrm{Ar}] 4 s^{2} 3 d^{7}$} & ${ }^{4} F$ & 7.86 \\
\hline 28 & $\mathrm{Ni}$ & {$[\mathrm{Ar}] 4 s^{2} 3 d^{8}$} & ${ }^{3} F$ & 7.633 \\
\hline 29 & $\mathrm{Cu}$ & {$[\mathrm{Ar}] 4 s 3 d^{10}$} & ${ }^{2} S$ & 7.724 \\
\hline 30 & $\mathrm{Zn}$ & {$[\mathrm{Ar}] 4 s^{2} 3 d^{10}$} & ${ }^{1} S$ & 9.391 \\
\hline 31 & Ga & {$[\mathrm{Ar}] 4 s^{2} 3 d^{10} 4 p$} & ${ }^{2} P$ & 6.00 \\
\hline 32 & $\mathrm{Ge}$ & {$[\mathrm{Ar}] 4 s^{2} 3 d^{10} 4 p^{2}$} & ${ }^{3} P$ & 7.88 \\
\hline 33 & As & {$[\mathrm{Ar}] 4 s^{2} 3 d^{10} 4 p^{3}$} & ${ }^{4} S$ & 9.81 \\
\hline 34 & $\mathrm{Se}$ & {$[\mathrm{Ar}] 4 s^{2} 3 d^{10} 4 p^{4}$} & ${ }^{3} P$ & 9.75 \\
\hline 35 & $\mathrm{Br}$ & {$[\mathrm{Ar}] 4 s^{2} 3 d^{10} 4 p^{5}$} & ${ }^{2} P$ & 11.84 \\
\hline
\end{tabular}


Table 1-5 (continued)

\begin{tabular}{|c|c|c|c|c|}
\hline$Z$ & Atom (A) & $\begin{array}{l}\text { Orbital electronic } \\
\text { configuration }\end{array}$ & $\begin{array}{c}\text { Ground } \\
\text { state } \\
\text { term }\end{array}$ & $I P_{I}, e V$ \\
\hline 36 & $\mathrm{Kr}$ & {$[\mathrm{Ar}] 4 s^{2} 3 d^{10} 4 p^{6}$} & ${ }^{1} S$ & 13.996 \\
\hline 37 & $\mathrm{Rb}$ & {$[\mathrm{Kr}] 5 \mathrm{~s}$} & ${ }^{2} S$ & 4.176 \\
\hline 38 & $\mathrm{Sr}$ & {$[\mathrm{Kr}] 5 s^{2}$} & ${ }^{1} S$ & 5.692 \\
\hline 39 & $\mathrm{Y}$ & {$[\mathrm{Kr}] 5 s^{2} 4 d$} & ${ }^{2} D$ & 6.5 \\
\hline 40 & $\mathrm{Zr}$ & {$[\mathrm{Kr}] 5 s^{2} 4 d^{2}$} & ${ }^{3} F$ & 6.95 \\
\hline 41 & $\mathrm{Nb}$ & {$[K r] 5 s \quad 4 d^{4}$} & ${ }^{6} \mathrm{D}$ & 6.77 \\
\hline 42 & Mo & {$[\mathrm{Kr}] 5 s \quad 4 d^{5}$} & ${ }^{7} S$ & 7.10 \\
\hline 43 & $\mathrm{Tc}$ & {$[\mathrm{Kr}] 5 s^{2} 4 d^{5}$} & ${ }^{6} S$ & 7.28 \\
\hline 44 & $\mathrm{Ru}$ & {$[\mathrm{Kr}] 5 s 4 d^{7}$} & ${ }^{5} F$ & 7.364 \\
\hline 45 & $\mathrm{Rh}$ & {$\left[\mathrm{Kr} / 5 s \quad 4 d^{8}\right.$} & ${ }^{4} F$ & 7.46 \\
\hline 46 & $\mathrm{Pd}$ & {$[\mathrm{Kr}] 4 d^{10}$} & ${ }^{1} S$ & 8.33 \\
\hline 47 & $\mathrm{Ag}$ & {$[\mathrm{Kr}] 5 s 4 d^{10}$} & ${ }^{2} S$ & 7.574 \\
\hline 48 & $\mathrm{Cd}$ & {$[\mathrm{Kr}] 5 s^{2} 4 d^{10}$} & ${ }^{1} S$ & 8.991 \\
\hline 49 & In & {$[\mathrm{Kr}] 5 s^{2} 4 d^{10} 5 p$} & ${ }^{2} P$ & 5.785 \\
\hline 50 & $\mathrm{Sn}$ & {$[\mathrm{Kr}] 5 s^{2} 4 d^{10} 5 p^{2}$} & ${ }^{3} P$ & 7.342 \\
\hline 51 & $\mathrm{Sb}$ & {$[\mathrm{Kr}] 5 s^{2} 4 d^{10} 5 p^{3}$} & ${ }^{4} S$ & 8.639 \\
\hline 52 & $\mathrm{Te}$ & {$[\mathrm{Kr}] 5 s^{2} 4 d^{10} 5 p^{4}$} & ${ }^{3} P$ & 9.01 \\
\hline 53 & I & {$[\mathrm{Kr}] 5 s^{2} 4 d^{10} 5 p^{5}$} & ${ }^{2} p$ & 10.454 \\
\hline 54 & $\mathrm{Xe}$ & {$[\mathbf{K} \mathbf{r}] 5 s^{2} 4 d^{10} 5 p^{6}$} & ${ }^{1} S$ & 12.127 \\
\hline 55 & $\mathrm{Cs}$ & {$[\mathrm{Xe}] 6 \mathrm{~s}$} & ${ }^{2} S$ & 3.893 \\
\hline 56 & $\mathrm{Ba}$ & {$[\mathrm{Xe}] 6 s^{2}$} & ${ }^{1} S$ & 5.210 \\
\hline 57 & $\mathrm{La}$ & {$[\mathrm{Xe}] 6 s^{2} 5 d$} & ${ }^{2} D$ & 5.61 \\
\hline 58 & $\mathrm{Ce}$ & {$[\mathrm{Xe}] 6 s^{2} 4 f 5 d$} & ${ }^{3} H$ & $6.91^{b}$ \\
\hline 59 & $\operatorname{Pr}$ & {$[\mathrm{Xe}] 6 s^{2} 4 f^{3}$} & ${ }^{4} \mathrm{I}$ & $5.76^{\mathrm{b}}$ \\
\hline 60 & $\mathrm{Nd}$ & {$[\mathrm{Xe}] 6 s^{2} 4 f^{4}$} & ${ }^{5} \mathrm{I}$ & $6.31^{b}$ \\
\hline 61 & $\mathrm{Pm}$ & {$[\mathrm{Xe}] 6 s^{2} 4 f^{5}$} & ${ }^{6} H$ & \\
\hline 62 & $\mathrm{Sm}$ & {$[\mathrm{Xe}] 6 s^{2} 4 f^{6}$} & ${ }^{7} F$ & $5.6^{\mathrm{b}}$ \\
\hline 63 & $\mathrm{Eu}$ & {$[\mathrm{Xe}] 6 s^{2} 4 f^{7}$} & ${ }^{8} S$ & $5.67^{\mathrm{b}}$ \\
\hline 64 & $\mathrm{Gd}$ & {$[\mathrm{Xe}] 6 s^{2} 4 f^{7} 5 d$} & ${ }^{9} D$ & $6.16^{\mathrm{b}}$ \\
\hline 65 & $\mathrm{~Tb}$ & {$[\mathrm{Xe}] 6 s^{2} 4 f^{9} ?$} & ${ }^{6} H$ & $6.74^{\mathrm{b}}$ \\
\hline 66 & Dy & {$[\mathrm{Xe}] 6 s^{2} 4 f^{10}$} & ${ }^{5} \mathrm{I}$ & $6.82^{b}$ \\
\hline 67 & Ho & {$[\mathrm{Xe}] 6 s^{2} 4 f^{1 I}$} & ${ }^{4} I$ & \\
\hline 68 & $\mathrm{Er}$ & {$[\mathrm{Xe}] 6 s^{2} 4 f^{\mathrm{I} 2}$} & ${ }^{3} H$ & $6.08^{\mathrm{c}}$ \\
\hline 69 & $\mathrm{Tm}$ & {$[\mathrm{Xe}] 6 s^{2} 4 f^{13}$} & ${ }^{2} F$ & $5.81^{\mathrm{d}}$ \\
\hline 70 & Yo & {$[\mathrm{Xe}] 6 s^{2} 4 f^{14}$} & ${ }^{1} S$ & $6.2^{b}$ \\
\hline 71 & $\mathrm{Lu}$ & {$[\mathrm{Xe}] 6 s^{2} 4 f^{14} 5 d$} & ${ }^{2} D$ & $5.0^{\mathrm{b}}$ \\
\hline 72 & $\mathrm{Hf}$ & {$[\mathrm{Xe}] 6 s^{2} 4 f^{14} 5 d^{2}$} & ${ }^{3} F$ & \\
\hline
\end{tabular}


Table $1-5$ (continued)

\begin{tabular}{|c|c|c|c|c|}
\hline$Z$ & Atom (A) & $\begin{array}{l}\text { Orbital electronic } \\
\text { configuration }\end{array}$ & $\begin{array}{l}\text { Ground } \\
\text { state } \\
\text { term }\end{array}$ & $I P_{1}, e V$ \\
\hline 73 & $\mathrm{Ta}$ & {$[\mathrm{Xe}] 6 s^{2} 4 f^{14} 5 d^{3}$} & ${ }^{4} F$ & 7.88 \\
\hline 74 & $W$ & {$[\mathrm{Xe}] 6 s^{2} 4 f^{14} 5 d^{4}$} & ${ }^{5} D$ & 7.98 \\
\hline 75 & $\mathrm{Re}$ & {$[\mathrm{Xe}] 6 s^{2} 4 f^{14} 5 d^{5}$} & ${ }^{6} S$ & 7.87 \\
\hline 76 & Os & {$[\mathrm{Xe}] 6 s^{2} 4 f^{14} 5 d^{6}$} & ${ }^{5} \mathrm{D}$ & 8.7 \\
\hline 77 & $\operatorname{Ir}$ & {$[\mathrm{Xe}] 6 s^{2} 4 f^{14} 5 d^{7}$} & ${ }^{4} F$ & 9 \\
\hline 78 & $\mathrm{Pt}$ & {$[\mathrm{Xe}] 6 s^{2} 4 f^{14} 5 d^{9 n}$} & ${ }^{3} D$ & 8.0 \\
\hline 79 & $\mathrm{Au}$ & [Xe]6s $4 f^{14} 5 d^{10}$ & ${ }^{2} S$ & 9.22 \\
\hline 80 & $\mathrm{Hg}$ & {$[\mathrm{Xe}] 6 s^{2} 4 f^{14} 5 d^{10}$} & ${ }^{1} S$ & 10.43 \\
\hline 81 & $\mathrm{Tl}$ & {$[\mathrm{Xe}] 6 s^{2} 4 f^{14} 5 d^{10} 6 p$} & ${ }^{2} P$ & 6.106 \\
\hline 82 & $\mathrm{~Pb}$ & {$[\mathrm{Xe}] 6 s^{2} 4 f^{14} 5 d^{10} 6 p^{2}$} & ${ }^{3} P$ & 7.415 \\
\hline 83 & $\mathrm{Bi}$ & {$[\mathrm{Xe}] 6 s^{2} 4 f^{14} 5 d^{10} 6 p^{3}$} & ${ }^{4} S$ & 7.287 \\
\hline 84 & $\mathrm{Po}$ & {$[\mathrm{Xe}] 6 s^{2} 4 f^{14} 5 d^{10} 6 p^{4}$} & ${ }^{3} P$ & 8.43 \\
\hline 85 & At & {$[\mathrm{Xe}] 6 s^{2} 4 f^{14} 5 d^{10} 6 p^{5}$} & ${ }^{2} P$ & \\
\hline 86 & $\mathrm{Rn}$ & {$[\mathrm{Xe}] 6 s^{2} 4 f^{14} 5 d^{10} 5 p^{6}$} & ${ }^{1} S$ & 10.746 \\
\hline 87 & $\mathrm{Fr}$ & {$[\mathrm{Rn}] 7 \mathrm{~s}$} & ${ }^{2} S$ & \\
\hline 88 & $\mathrm{Ra}$ & {$[\operatorname{Rn}] 7 s^{2}$} & ${ }^{1} S$ & 5.277 \\
\hline 89 & $\mathrm{Ac}$ & {$[\mathrm{Rn}] 7 s^{2} 6 d$} & ${ }^{2} D$ & \\
\hline 90 & Th & {$[\mathrm{Rn}] 7 s^{2} 6 d^{2}$} & ${ }^{3} F$ & $6.95^{\mathrm{e}}$ \\
\hline 91 & $\mathrm{~Pa}$ & {$[\mathrm{Rn}] 7 s^{2} 5 f^{2} 6 d$} & ${ }^{4} K$ & \\
\hline 92 & $\mathrm{U}$ & {$[\operatorname{Rn}] 7 s^{2} 5 f^{3} 6 d$} & ${ }^{5} L$ & $6.1^{\mathrm{e}}$ \\
\hline 93 & $\mathrm{~Np}$ & {$[\mathrm{Rn}] 7 s^{2} 5 f^{4} 6 d$} & ${ }^{6} \mathrm{~L}$ & \\
\hline 94 & $\mathrm{Pu}$ & {$[\operatorname{Rn}] 7 s^{2} 5 f^{6}$} & ${ }^{7} F$ & $5.1^{\mathrm{f}}$ \\
\hline 95 & $\mathrm{Am}$ & {$[\operatorname{Rn}] 7 s^{2} 5 f^{7}$} & ${ }^{8} S$ & $6.0^{g}$ \\
\hline 96 & $\mathrm{Cm}$ & {$[\operatorname{Rn}] 7 s^{2} 5 f^{7} 6 d$} & ${ }^{9} D$ & \\
\hline 97 & $\mathrm{Bk}$ & {$[\operatorname{Rn}] 7 s^{2} 5 f^{9}$} & ${ }^{6} H$ & \\
\hline 98 & $\mathrm{Cf}$ & {$[\operatorname{Rn}] 7 s^{2} 5 f^{10}$} & ${ }^{5} \mathrm{I}$ & \\
\hline 99 & $\mathrm{Es}$ & {$[\operatorname{Rn}] 7 s^{2} 5 f^{11}$} & ${ }^{4} I$ & \\
\hline 100 & $\mathrm{Fm}$ & {$[\operatorname{Rn}] 7 s^{2} 5 f^{12}$} & ${ }^{3} H$ & \\
\hline 101 & Md & {$[\operatorname{Rn}] 7 s^{2} 5 f^{13}$} & ${ }^{2} F$ & \\
\hline 102 & No & {$[\operatorname{Rn}] 7 s^{2} 5 f^{14}$} & ${ }^{1} S$ & \\
\hline 103 & $\mathrm{Lw}$ & {$[\operatorname{Rn}] 7 s^{2} 5 f^{14} 6 d$} & ${ }^{2} D$ & \\
\hline
\end{tabular}

a From C. E. Moore, "Atomic Energy Levels," NBS Circular 467, 1949, 1952 , and 1958, except as indicated.

${ }^{\mathrm{b}} \mathrm{T}$. Moeller, The Chemistry of the Lanthanides, Reinhold, New York, 1963, p. 37.

${ }^{c}$ N. I. Ionov and M. A. Mitsev, Zhur. Eksptl. i Theoret. Fiz., 40, 741(1961).

d. Blaise and R. Vetter, Compt. Rend., 256, 630 (1963).

K. F. Zmbov, Bull. Boris Kidrich Inst. Nucl. Sci., 13, 17 (1962).

${ }^{\mathrm{f}}$ R.H.U.M. Dawton and K. L. Wilkinson, Atomic Energy Research Estab. (Gt. Brit.), GR/R, 1906 (1956).

${ }^{\mathrm{M}} \mathrm{M}$. Fred and F. S. Tompkins, J. Opt. Soc. Am., 47, 1076 (1957). 
from a neutral atom is easier than its removal from a positively charged ion.

In any column in the periodic table, the IP's decrease as the atomic number increases. Let us examine, for example, the $\mathrm{Li}$ and $\mathrm{Cs}$ atoms. Lithium, which has $\mathrm{IP}_{1}=5.390 \mathrm{eV}$, has the electronic configuration $[\mathrm{He}] 2 s$. Cesium, with $\mathrm{IP}_{1}=3.893 \mathrm{eV}$, has the structure $[\mathrm{Xe}] 6 s$. The $2 s$ electron in $\mathrm{Li}$ spends much more time near the nucleus than the $6 s$ electron does in Cs. This means that the net attraction between the electron and $Z_{\text {eff }}$, the shielded nuclear charge, is substantially larger for the $\mathrm{Li} 2 s$ electron than for the Cs $6 s$ electron, a fact that is illustrated in Fig. 1-9.

In any row in the periodic table, the IP's generally increase from left to right, being smallest for the alkali metal atoms and largest for the inert gas atoms. There are irregularities, however, since atoms

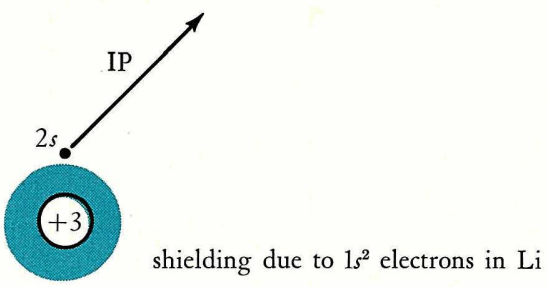

(a)

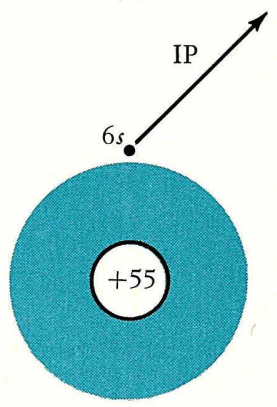

shielding due to $1 s^{2} 2 s^{2} 2 p^{6} 3 s^{2} 3 p^{6} 4 s^{2} 3 d^{10} 4 p^{6} 5 s^{2} 4 d^{10} 5 p^{6}$ electrons

(b)

Figure 1-9 Ionization of an electron from $(a)$ a lithium atom and $(b)$ a cesium atom. 
with filled or half-filled subshells have larger IP's than might be expected. For example, $\mathrm{Be}\left([\mathrm{He}] 2 s^{2}\right)$ has $\mathrm{IP}_{1}=9.320 \mathrm{eV}$ and $\mathrm{B}\left([\mathrm{He}] 2 s^{2} 2 p^{1}\right)$ has $\mathrm{IP}_{1}=8.296 \mathrm{eV} ; \mathrm{N}\left([\mathrm{He}] 2 s^{2} 2 p^{3}\right)$ has $\mathrm{IP}_{1}=14.54 \mathrm{eV}$ and $\mathrm{O}\left([\mathrm{He}] 2 s^{2} 2 p^{4}\right)$ has $\mathrm{IP}_{1}=13.614 \mathrm{eV}$. The steady if slightly irregular increase in IP's from $\mathrm{Li}\left(\mathrm{IP}_{1}=5.390 \mathrm{eV}\right)$ to $\mathrm{Ne}\left(\mathrm{IP}_{1}=21.559\right.$ $\mathrm{eV}$ ) is due to the steady increase in $Z_{\text {eff }}$ observed between $\mathrm{Li}$ and $\mathrm{Ne}$. The electrons added from $\mathrm{Li}$ to $\mathrm{Ne}$ all enter $2 s$ and $2 p$ orbitals and are not able to completely shield each other from the increasing nuclear charge.

The variation of the ionization potential of atoms with atomic number is shown in Fig. 1-10.

\section{1-17 ELECTRON AFFinities .}

The electron affinity (abbreviated EA) of an atom is the energy released (or needed, if the atom has a negative EA) when the atom

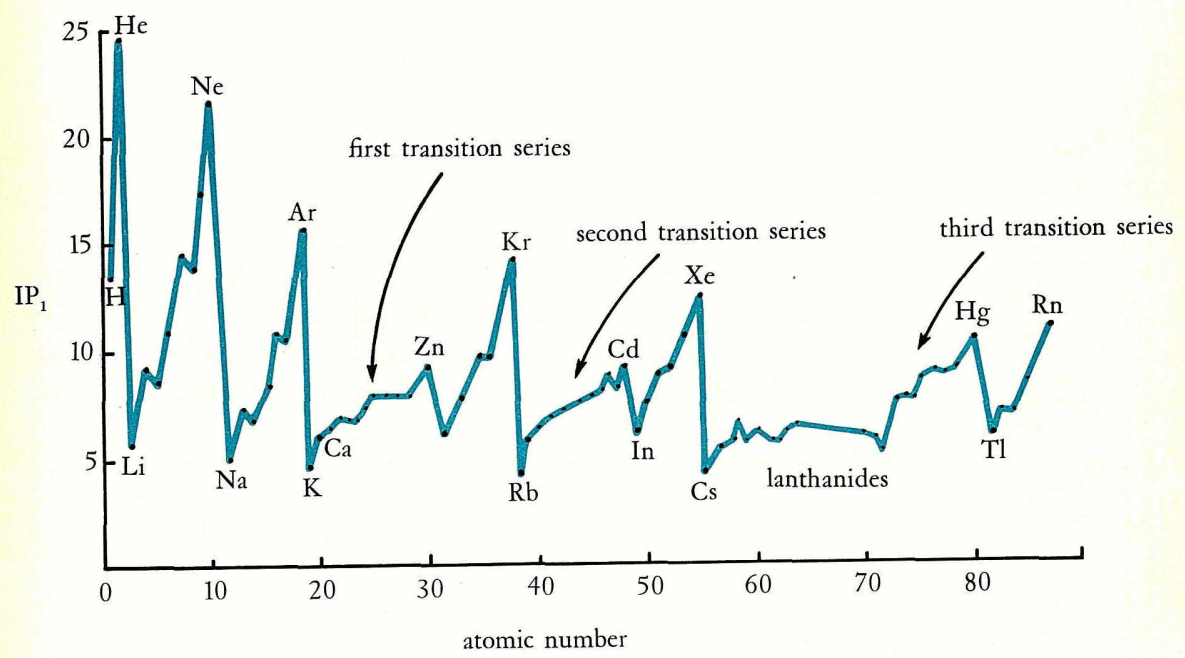

Figure 1-10 Variation of atomic ionization potential with atomic number. 
adds an extra electron to give a negative ion. Thus we have the equation

$$
\text { atom }+ \text { electron } \rightarrow \text { uninegative ion }+\mathrm{EA}(\text { energy })
$$

$$
\text { Table } 1-6
$$

\section{Atomic Electron Affinities}

\begin{tabular}{llll}
\hline Atom (A) & $\begin{array}{c}\text { Orbital electronic } \\
\text { configuration }\end{array}$ & $\begin{array}{l}\text { EA, eV } \\
\text { configuration of } A^{-}\end{array}$ \\
\hline $\mathrm{H}$ & $1 s$ & $0.747^{\mathrm{a}}$ & $\mathrm{He}$ \\
$\mathrm{F}$ & {$[\mathrm{He}] 2 s^{2} 2 p^{5}$} & $3.45^{\mathrm{b}}$ & $\mathrm{Ne}$ \\
$\mathrm{Cl}$ & {$[\mathrm{Ne}] 3 s^{2} 3 p^{5}$} & $3.61^{\mathrm{b}}$ & $\mathrm{Ar}$ \\
$\mathrm{Br}$ & {$[\mathrm{Ar}] 4 s^{2} 3 d^{10} 4 p^{5}$} & $3.36^{\mathrm{b}}$ & $\mathrm{Kr}$ \\
$\mathrm{I}$ & {$[\mathrm{Kr}] 5 s^{2} 4 d^{10} 5 p^{5}$} & $3.06^{\mathrm{b}}$ & $\mathrm{Xe}$ \\
$\mathrm{O}$ & {$[\mathrm{He}] 2 s^{2} 2 p^{4}$} & $1.47^{\mathrm{c}}$ & {$[\mathrm{He}] 2 s^{2} 2 p^{5}$} \\
$\mathrm{~S}$ & {$[\mathrm{Ne}] 3 s^{2} 3 p^{4}$} & $2.07^{\mathrm{d}}$ & {$[\mathrm{Ne}] 3 s^{2} 3 p^{5}$} \\
$\mathrm{Se}$ & {$[\mathrm{Ar}] 4 s^{2} 3 d^{10} 4 p^{4}$} & $(1.7)^{\mathrm{e}}$ & {$[\mathrm{Ar}] 4 s^{2} 3 d^{10} 4 p^{5}$} \\
$\mathrm{Te}$ & {$[\mathrm{Kr}] 5 s^{2} 4 d^{10} 5 p^{4}$} & $(2.2)^{\mathrm{e}}$ & {$[\mathrm{Kr}] 5 s^{2} 4 d^{10} 5 p^{5}$} \\
$\mathrm{~N}$ & {$[\mathrm{He}] 2 s^{2} 2 p^{3}$} & $(-0.1)^{\mathrm{f}}$ & {$[\mathrm{He}] 2 s^{2} 2 p^{4}$} \\
$\mathrm{P}$ & {$[\mathrm{Ne}] 3 s^{2} 3 p^{3}$} & $(0.7)^{\mathrm{f}}$ & {$[\mathrm{Ne}] 3 s^{2} 3 p^{4}$} \\
$\mathrm{As}$ & {$[\mathrm{Ar}] 4 s^{2} 3 d^{10} 4 p^{3}$} & $(0.6)^{\mathrm{f}}$ & {$[\mathrm{Ar}] 4 s^{2} 3 d^{10} 4 p^{4}$} \\
$\mathrm{C}$ & {$[\mathrm{He}] 2 s^{2} 2 p^{2}$} & $1.25^{\mathrm{g}}$ & {$[\mathrm{He}] 2 s^{2} 2 p^{3}$} \\
$\mathrm{Si}$ & {$[\mathrm{Ne}] 3 s^{2} 3 p^{2}$} & $(1.63)^{\mathrm{f}}$ & {$[\mathrm{Ne}] 3 s^{2} 3 p^{3}$} \\
$\mathrm{Ge}$ & {$[\mathrm{Ar}] 4 s^{2} 3 d^{10} 4 p^{2}$} & $(1.2)^{\mathrm{f}}$ & {$[\mathrm{Ar}] 4 s^{2} 3 d^{10} 4 p^{3}$} \\
$\mathrm{~B}$ & {$[\mathrm{He}] 2 s^{2} 2 p$} & $(0.2)^{\mathrm{a}}$ & {$[\mathrm{He}] 2 s^{2} 2 p^{2}$} \\
$\mathrm{Al}$ & {$[\mathrm{Ne}] 3 s^{2} 3 p$} & $(0.6)^{\mathrm{a}}$ & {$[\mathrm{Ne}] 3 s^{2} 3 p^{2}$} \\
$\mathrm{Ga}$ & {$[\mathrm{Ar}] 4 s^{2} 3 d^{10} 4 p$} & $(0.18)^{\mathrm{f}}$ & {$[\mathrm{Ar}] 4 s^{2} 3 d^{10} 4 p^{2}$} \\
$\mathrm{In}$ & {$[\mathrm{Kr}] 5 s^{2} 4 d^{10} 5 p$} & $(0.2)^{\mathrm{f}}$ & {$[\mathrm{Kr}] 5 s^{2} 4 d^{10} 5 p^{2}$} \\
$\mathrm{Be}$ & {$[\mathrm{He}] 2 s^{2}$} & $(-0.6)^{\mathrm{a}}$ & {$[\mathrm{He}] 2 s^{2} 2 p$} \\
$\mathrm{Mg}$ & {$[\mathrm{Ne}] 3 s^{2}$} & $(-0.3)^{\mathrm{a}}$ & {$[\mathrm{Ne}] 3 s^{2} 3 p$} \\
$\mathrm{Li}$ & {$[\mathrm{He}] 2 s$} & $(0.54)^{\mathrm{a}}$ & {$[\mathrm{He}] 2 s^{2}$} \\
$\mathrm{Na}$ & {$[\mathrm{Ne}] 3 s$} & $(0.74)^{\mathrm{a}}$ & {$[\mathrm{Ne}] 3 s^{2}$} \\
$\mathrm{Zn}$ & {$[\mathrm{Ar}] 4 s^{2} 3 d^{10}$} & $(-0.9)^{\mathrm{f}}$ & {$[\mathrm{Ar}] 4 s^{2} 3 d^{10} 4 p$} \\
$\mathrm{Cd}$ & {$[\mathrm{Kr}] 5 s^{2} 4 d^{10}$} & $(-0.6)^{\mathrm{f}}$ & {$[\mathrm{Kr}] 5 s^{2} 4 d^{10} 5 p$} \\
\hline
\end{tabular}

${ }^{\mathrm{a}}$ H. A. Skinner and H. O. Pritehard, Trans. Faraday Soc., 49, 1254 (1953).

${ }^{b}$ R. S. Berry and C. W. Riemann, J. Chem. Phys., 38, 1540 (1963).

${ }^{\mathrm{C}}$ L. M. Branscomb, Nature, 182, 248 (1958).

d L. M. Branscomb and S. J. Smith, J. Chem. Phys., 25, 598 (1956).

${ }^{\text {e}}$ H. O. Pritchard, Chem.Revs., 52, 529 (1953).

${ }^{f}$ A. P. Ginsburg and J. M. Miller, J. morg. Nucl. Chem., 7, 351 (1958).

${ }^{8}$ M. L. Seman and L. M. Branscomb, Phys. Rev., 125, 1602 (1962). 
Unfortunately, as a result of certain experimental difficulties, very few EA values are precisely known. A representative list is given in Table 1-6.

The halogen atoms have relatively large EA's, since the resulting halide ions have a stable filled-shell electronic configuration. Atoms with filled subshells often have negative EA values. Good examples are $\mathrm{Be}, \mathrm{Mg}$, and $\mathrm{Zn}$.

It is interesting to note that the atoms in the nitrogen family, with the electronic configuration $s^{2} p^{3}\left({ }^{4} S\right)$, have very small EA's. Thus we have additional evidence for the greater stability of a half-filled subshell.

\section{SUPPLEMENTARY PROBLEMS}

1(a). Compare the velocity and radius of an electron in the fourth Bohr orbit with the velocity and radius of an electron in the first Bohr orbit; (b) Derive the expression, dependent only on the variable $n$, for the velocity of an electron in a Bohr orbit.

2. Calculate the energy of an electron in the Bohr orbit with $n=3$.

3. Calculate the second ionization potential of He.

4. Calculate the frequencies of the first three lines in the Lyman series (the lowest-frequency lines).

5. The Balmer series in the spectrum of the hydrogen atom arises from transitions from higher levels to $n=2$. Find which of the Balmer lines fall in the visible region of the spectrum (visible light wavelengths are between 4000 and $7000 \mathrm{~A}$ ).

6. Following the Pauli principle and Hund's first rule, give the orbital configuration and the number of unpaired electrons in the ground state for the following atoms: (a) N; (b) $\mathrm{S}$; (c) Ca; (d) Fe; (e) $\mathrm{Br}$.

7. Find the terms for the following orbital configurations, and in each case designate the term of lowest energy: (a) $2 s$; (b) $2 p^{3}$; (c) $2 p^{2} 3 s$; (d) $2 p 3 p$; (e) $2 p 3 d$; (f) $3 d^{3}$; (g) $3 d^{5}$; (h) $3 d^{9}$; (i) $2 s 4 f$; (j) $2 p^{5}$; (k) $3 d^{3} 4 s$.

8. Find the ground-state term for the following atoms: (a) $\mathrm{Si}$; (b) $\mathrm{Mn}$; (c) $\mathrm{Rb}$; (d) $\mathrm{Ni}$. 


\section{II \\ Diatomic Molecules}

\section{2-1 COVALENT BONDING}

A molecule is any stable combination of more than one atom. 1 The simplest neutral molecule is a combination of two hydrogen atoms, which we call the hydrogen molecule or $\mathrm{H}_{2}$. The $\mathrm{H}_{2}$ molecule is homonuclear, since both atomic nuclei used in forming the molecule are the same.

The forces that hold two hydrogen atoms together in the $\mathrm{H}_{2}$ molecule are described collectively by the word bond. We know this bond to be quite strong, since at ordinary temperatures hydrogen exists in the form $\mathrm{H}_{2}$, not $\mathrm{H}$ atoms. Only at very high temperatures is $\mathrm{H}_{2}$ broken up into its $\mathrm{H}$ atom components. Let us try to visualize the bonding in $\mathrm{H}_{2}$ by allowing two hydrogen atoms to approach each other, as illustrated in Fig. 2-1. When the atoms are at close range, two electrostatic forces become important: first, the attraction between the nucleus $\mathbb{H}_{a}$ and the electron associated with $1 s_{b}$, as well as that between the nucleus $\mathrm{H}_{b}$ and the electron associated with $1 s_{a}$; and second, the repulsion between $\mathrm{H}_{a}$ and $\mathrm{H}_{b}$ as well as that between $1 s_{a}$ and $1 s_{b}$.

The attractive term is more important at large $\mathrm{H}_{a}-\mathrm{H}_{b}$ distances, but the situation changes as the two atoms come closer together, the importance of the $\mathrm{H}_{a}-\mathrm{H}_{b}$ repulsion increasing as internuclear distances become very short. This state of affairs is described by an 36 


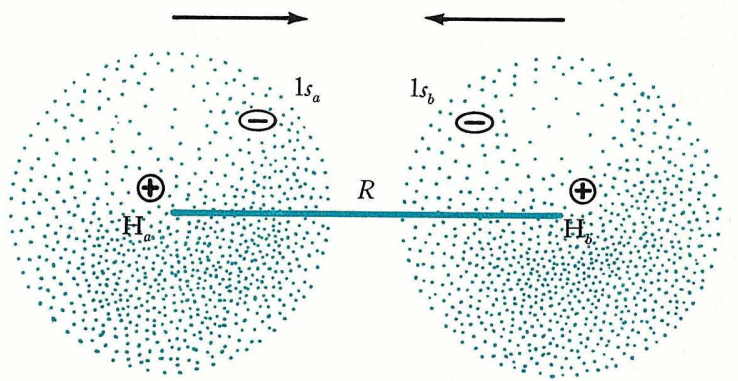

Figure 2-1 Schematic drawing of two hydrogen atoms approaching each other.

energy curve such as that shown in Fig. 2-2. The energy of the system falls until the $\mathrm{H}_{a}-\mathrm{H}_{b}$ repulsion at very short ranges forces the energy back up again. The minimum in the curve gives both the most stable internuclear separation in the $\mathrm{H}_{2}$ molecule and its gain in stability over two isolated $H$ atoms.

One of the early successful pictures of a chemical bond involving electrons and nuclei resulted from the work of the American physical chemist, G. N. Lewis. Lewis formulated the electron-pair bond, in which the combining atoms tend to associate themselves with just enough electrons to achieve an inert-gas electronic configuration. The hydrogen molecule is, in the Lewis theory, held together by an electron-pair bond (Fig. 2-3). Each hydrogen has the same partial claim to the electron pair and thus achieves the stable $1 s^{2}$ helium configuration. A bond in which the electrons are equally shared by the participating nuclei is called a covalent bond.

The remainder of this book will be devoted to the modern ideas of bonding in several important classes of molecules. The emphasis will be on the molecular-orbital theory, with comparisons made from time to time to the valence-bond theory. Of the many scientists involved in the development of these theories, the names of R. S. Mulliken (molecular-orbital theory) and Linus Pauling (valencebond theory) are particularly outstanding. 


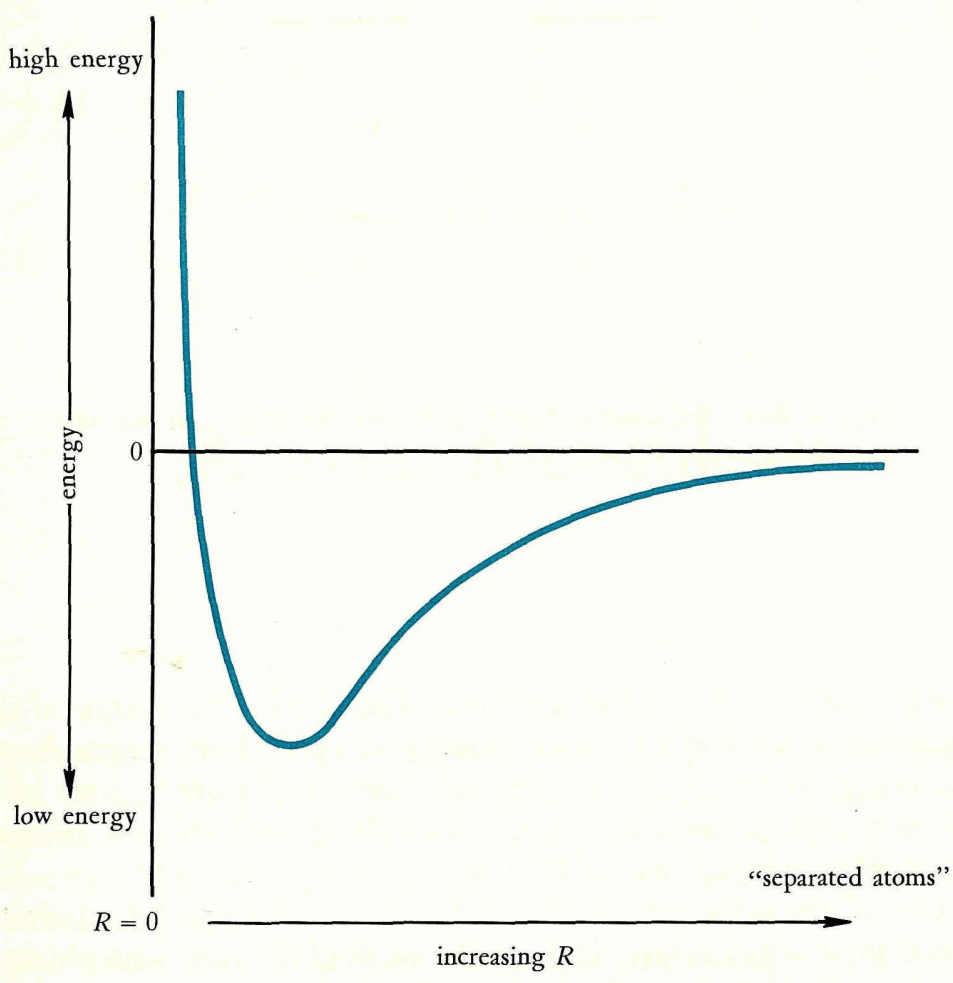

Figure 2-2 Energy of a system of two hydrogen atoms as a function of internuclear separation.

\section{2-2 MOLECULAR-ORBITAL THEORY}

According to molecular-orbital theory, electrons in molecules are in orbitals that may be associated with several nuclei. Molecular orbitals in their simplest approximate form are considered to be linear combinations of atomic orbitals. We assume that when an electron in a molecule is near one particular nucleus, the molecular wave function is approximately an atomic orbital centered at that nucleus. This means that we can form molecular orbitals by simply adding and subtracting appropriate atomic orbitals. The method is usually abbreviated LCAO-MO, which stands for linear combination of atomic 


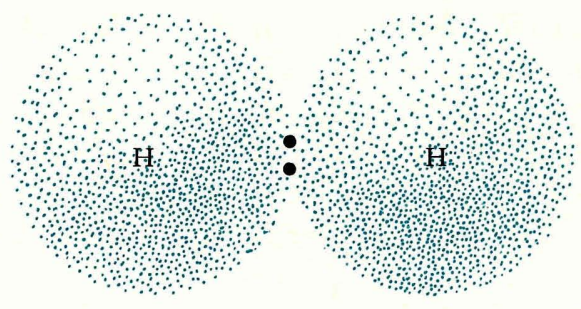

or

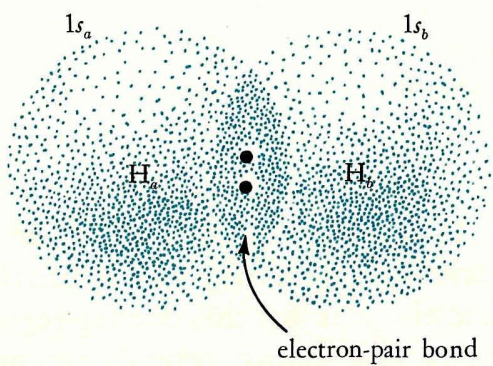

Figure 2-3 Electron-pair bond in the hydrogen molecule.

orbitals-molecular orbitals. We shall use the abbreviation MO in this text for a molecular orbital.

Atomic orbitals that are in the proper stability range to be used in bonding are called valence orbitals. The valence orbitals of an atom are those that have accepted electrons since the last inert gas and, in addition, any others in the stability range of the orbitals that will be encountered before the next inert gas. For example, the valence orbital of the hydrogen atom is $1 s$. The $2 s$ and $2 p$ orbitals of hydrogen are too high in energy to be used in strong bonding.

\section{2-3 BONDING AND ANTIBONDING MOLECULAR ORBITALS}

Let us consider now the MO bonding scheme for the simplest imaginable molecule, one with two protons and one electron. This combination is $\mathrm{H}_{2}{ }^{+}$, the hydrogen molecule-ion. Each hydrogen in 


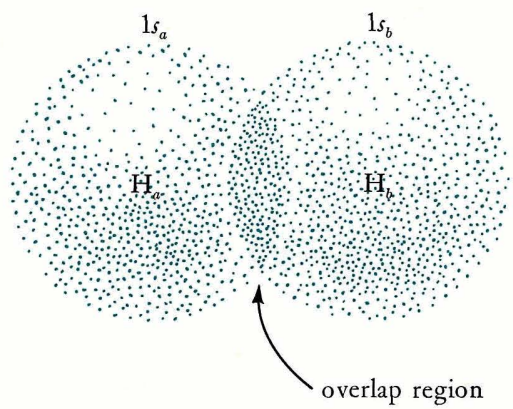

Figure 2-4 The overlap of two hydrogen $1 s$ orbitals in $\mathrm{H}_{2}{ }^{+}$.

the molecule has a $1 s$ valence orbital, as shown in Fig. 2-4. Notice that the two atomic orbitals overlap in the heavily shaded region between the two nuclei. It is just this overlap region that is affected by adding and subtracting atomic orbitals to construct molecular orbitals.

There are two different ways in which we can linearly combine two $1 s$ hydrogen atomic orbitals. The first is to add them together (Fig. 2-5). It is easy to see from this figure that an electron in MO I will spend most of its time in the overlap region between the nuclei $\mathrm{H}_{a}$ and $\mathrm{H}_{b}$. This maximizes the attractive force between the electron and the two nuclei; therefore an electron in this $\mathrm{MO}$ is more
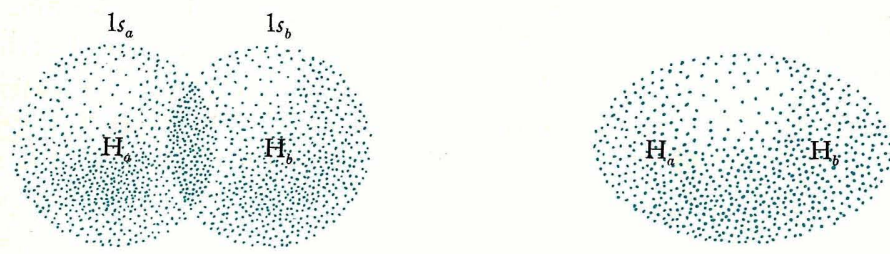

$$
1 s_{a}+1 s_{b}=\text { MO I }
$$

Figure 2-5 Schematic drawing of the formation of the bonding $\mathrm{MO}$ of $\mathrm{H}_{2}{ }^{+}$. 
stable than in either isolated $1 s$ atomic orbital. We refer to such an $\mathrm{MO}$ as bonding. Furthermore, this $\mathrm{MO}$ is symmetric for rotation about a line joining the two $\mathrm{H}$ nuclei. That is, if we place an arrow through the two nuclei, and then turn the arrow, the MO still looks exactly the same (Fig. 2-6). We call an orbital with such cylindrical symmetry a $\sigma$ molecular orbital. ${ }^{1}$ The $\sigma$ bonding $\mathrm{MO}$ will be abbreviated $\sigma^{b}$.

The other linear combination is formed by subtraction of one of the two hydrogen $1 s$ orbitals from the other (Fig. 2-7). This type of MO has a node in the region between the two nuclei. Thus an electron in MO II will never be found halfway between the two nuclei; instead it will be mainly confined to space outside the overlap region. An electron in MO II is less stable than in an isolated $1 s$ hydrogen atomic orbital, and we therefore say that II is antibonding. The antibonding MO also has cylindrical symmetry and thus is $\sigma$ antibonding or $\sigma^{*}$.
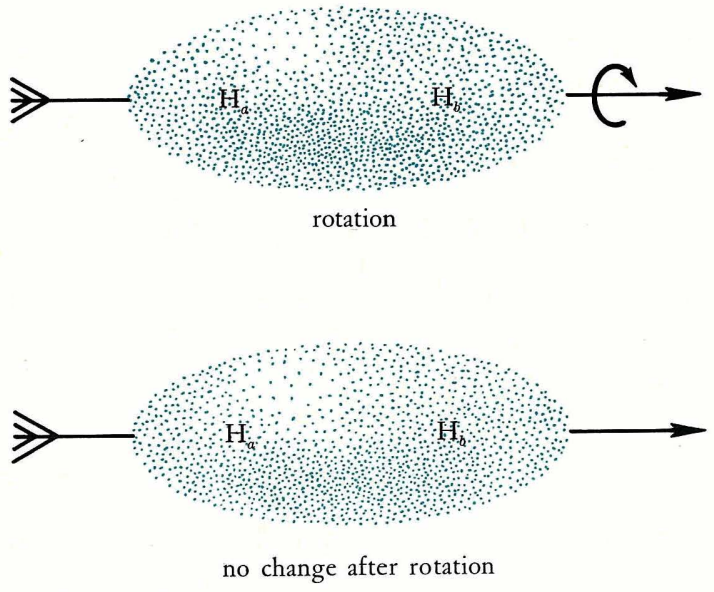

Figure 2-6 Rotation of the bonding $\mathrm{MO}$ of $\mathrm{H}_{2}{ }^{+}$about the internuclear axis.

${ }^{1}$ In fact, any molecular orbital that does not have a nodal plane containing the internuclear axis is a $\sigma$ molecular orbital. 


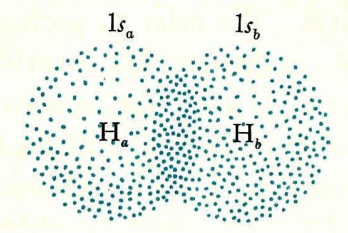

$1 s_{a}-1 s_{b} \quad=\quad$ MO II

Figure 2-7 Schematic drawing of the formation of the antibonding $\mathrm{MO}$ of $\mathrm{H}_{2}{ }^{+}$.

\section{2-4 MOLECULAR-ORBITAL ENERGY LEVELS}

The approximate wave functions for the $\sigma^{b}$ and $\sigma^{*}$ molecular orbitals are:

$$
\begin{aligned}
& \psi\left(\sigma^{b}\right)=N^{b}\left(1 s_{a}+1 s_{b}\right) \\
& \psi\left(\sigma^{*}\right)=N^{*}\left(1 s_{a}-1 s_{b}\right)
\end{aligned}
$$

Equations (2-1) and (2-2) are simply the analytical expressions for the molecular orbitals shown in Figs. $2-5$ and $2-7$, respectively. The values of the constants $N^{b}$ and $N^{*}$ in Eqs. (2-1) and (2-2) are fixed by the normalization condition,

$$
\int|\psi|^{2} d x d y d z=\int|\psi|^{2} d \tau=1
$$

Let us proceed to evaluate $N^{b}$. First we substitute $\psi\left(\sigma^{b}\right)$ in Eq. (2-3), giving

$$
\begin{aligned}
\int\left[\psi\left(\sigma^{b}\right)\right]^{2} d \tau=1 & \left.=\int N^{b}\left(1 s_{a}+1 s_{b}\right)\right]^{2} d \tau \\
= & \left(N^{b}\right)^{2}\left[\boldsymbol{S}\left(1 s_{a}\right)^{2} d \tau+\mathcal{S}\left(1 s_{b}\right)^{2} d \tau\right. \\
& \left.+2 \boldsymbol{S}\left(1 s_{a}\right)\left(1 s_{b}\right) d \tau\right]
\end{aligned}
$$

Provided the atomic orbitals $1 s_{a}$ and $1 s_{b}$ are already normalized,

$$
\mathcal{S}\left(1 s_{a}\right)\left(1 s_{a}\right) d \tau=\mathcal{S}\left(1 s_{b}\right)\left(1 s_{b}\right) d \tau=1
$$

The integral involving both $1 s_{a}$ and $1 s_{b}$ is called the overlap integral and is denoted by the letter $S$ :

$$
S=\text { overlap integral }=\mathcal{S}\left(1 s_{a}\right)\left(1 s_{b}\right) d \tau
$$


Thus, Eq. (2-4) reduces to

$$
\left(N^{b}\right)^{2}[2+2 S]=1
$$

and

$$
N^{b}= \pm \sqrt{\frac{1}{2(1+S)}}
$$

In our approximate scheme we shall neglect the overlap integral in determining the normalization constant. ${ }^{1}$ Therefore, arbitrarily picking the positive sign in Eq. (2-8), we have

$$
N^{b}=\sqrt{\frac{1}{2}}
$$

The value of $N^{*}$ is obtained in the same fashion, by substituting Eq. (2-2) in Eq. (2-3) and solving for $N^{*}$. The result is

$$
N^{*}= \pm \sqrt{\frac{1}{2(1-S)}}
$$

or, with the $S=0$ approximation,

$$
N^{*}=\sqrt{\frac{1}{2}}
$$

The approximate molecular orbitals for $\mathrm{H}_{2}{ }^{+}$are therefore

$$
\begin{aligned}
& \psi\left(\sigma^{b}\right)=\frac{1}{\sqrt{2}}\left(1 s_{a}+1 s_{b}\right) \\
& \psi\left(\sigma^{*}\right)=\frac{1}{\sqrt{2}}\left(1 s_{a}-1 s_{b}\right)
\end{aligned}
$$

The energies of these molecular orbitals are obtained from the Schrödinger equation,

$$
\pi \mathcal{K} \psi=E \psi
$$

Multiplying both sides of Eq. (2-14) by $\psi$ and then integrating, we have

$$
\int \psi \mathcal{H} \psi d \tau=E \int \psi^{2} d \tau
$$

1 This approximation involves a fairly substantial error in the case of $\mathrm{H}_{2}{ }^{+}$. The overlap of $1 s_{a}$ and $1 s_{b}$ in $\mathrm{H}_{2}{ }^{+}$is 0.590 . Thus we calculate $N^{b}=0.560$, as compared to $N^{b}=0.707$ for the $S=0$ approximation. In most other cases, however, the overlaps are smaller (usually between 0.2 and 0.3 ) and the approximation involves only a small error. 
Since $\int \psi^{2} d \tau=1$, Eq. (2-15) reduces to

$$
E=\int \mathcal{F} \mathcal{T} \psi d \tau
$$

Substituting Eq. (2-12) in Eq. (2-16), we have

$$
\begin{aligned}
& E\left[\psi\left(\sigma^{b}\right)\right]=\int\left[\psi\left(\sigma^{b}\right)\right] \mathfrak{H C}\left[\psi\left(\sigma^{b}\right)\right] d \tau=\frac{1}{2} \int\left(1 s_{a}+1 s_{b}\right) \mathcal{H C}\left(1 s_{a}+1 s_{b}\right) d \tau \\
& =\frac{1}{2} \int\left(1 s_{a}\right) \mathcal{H C}\left(1 s_{a}\right) d \tau+\frac{1}{2} \int\left(1 s_{b}\right) \mathcal{H C}\left(1 s_{b}\right) d \tau \\
& +\frac{1}{2} \int\left(1 s_{a}\right) \mathfrak{H}\left(1 s_{b}\right) d \tau+\frac{1}{2} \int\left(1 s_{b}\right) \mathcal{H}\left(1 s_{b}\right) d \tau
\end{aligned}
$$

We shall not attempt to evaluate the various integrals in Eq. (2-17), but instead shall replace them using the following shorthand:

$$
\begin{aligned}
& q_{a}=\mathscr{S}\left(1 s_{a}\right) \mathfrak{H C}\left(1 s_{a}\right) d \tau \\
& q_{b}=\mathscr{N}\left(1 s_{b}\right) \mathfrak{H C}\left(1 s_{b}\right) d \tau \\
& \beta=\mathscr{N}\left(1 s_{a}\right) \mathfrak{H C}\left(1 s_{b}\right) d \tau=\mathscr{N}\left(1 s_{b}\right) \mathfrak{H C}\left(1 s_{a}\right) d \tau
\end{aligned}
$$

In this case, since $1 s_{a}$ and $1 s_{b}$ are equivalent atomic orbitals,

$$
q_{a}=q_{b}=q
$$

We shall call $q_{a}$ and $q_{b}$ coulomb integrals. The coulomb integral represents the energy required to remove an electron from the valence orbital in question, in the field of the nuclei and other electrons in the molecule. Thus it is sometimes referred to as a valence ionization potential.

We shall call $\beta$ the excbange integral in this text. In other sources, however, you may find $\beta$ referred to as a resonance or covalent integral. We have seen that an electron in the $\sigma^{b}$ molecular orbital spends most of its time in the overlap region common to both nuclei. Thus the electron is stabilized in this favorable position for nucleus $a$-electronnucleus $b$ attractions. The exchange integral $\beta$ simply represents this added covalent-bonding stability.

Simplifying Eq. (2-17), we have finally

$$
E\left[\psi\left(\sigma^{b}\right)\right]=q+\beta
$$

The energy of the $\sigma^{*}$ molecular orbital is found in the same manner, substitution in Eq. (2-16) giving

$$
E\left[\psi\left(\sigma^{*}\right)\right]=\frac{1}{2} \mathcal{S}\left(1 s_{a}-1 s_{b}\right) \mathfrak{H}\left(1 s_{a}-1 s_{b}\right) d \tau=q-\beta
$$


This result shows that the antibonding molecular orbital is less stable than the bonding molecular orbital by an amount equal to $-2 \beta$. An electron in the $\sigma^{*}$ molecular orbital has only a small probability of being found in the energetically favored overlap region. Instead it is confined to the extreme ends of the molecule, which are positions of high energy relative to the middle of the molecule.

It is convenient to show the relative molecular-orbital energies in a diagram. Such a diagram for $\mathrm{H}_{2}{ }^{+}$is shown in Fig. 2-8. The valence orbitals of the combining atoms are represented in the outside columns and are ordered in terms of their coulomb energy. The most stable valence orbitals are placed lowest in the diagram. Since $1 s_{a}$ and $1 s_{b}$ have the same coulomb energy, these levels are placed directly opposite one another.

The molecular-orbital energies are indicated in the middle column. The $\sigma^{b}$ orbital is shown to be more stable than the combining $1 s$ valence orbitals, and the $\sigma^{*}$ orbital is shown to be correspondingly less stable.

The electron in the ground state of $\mathrm{H}_{2}{ }^{+}$occupies the more stable molecular orbital; that is,

$$
\text { ground state of } \mathrm{H}_{2}{ }^{+}=\sigma^{b}
$$

$$
\mathrm{H}_{a} \text { orbital molecular orbitals } \quad \mathrm{H}_{b} \text { orbital }
$$

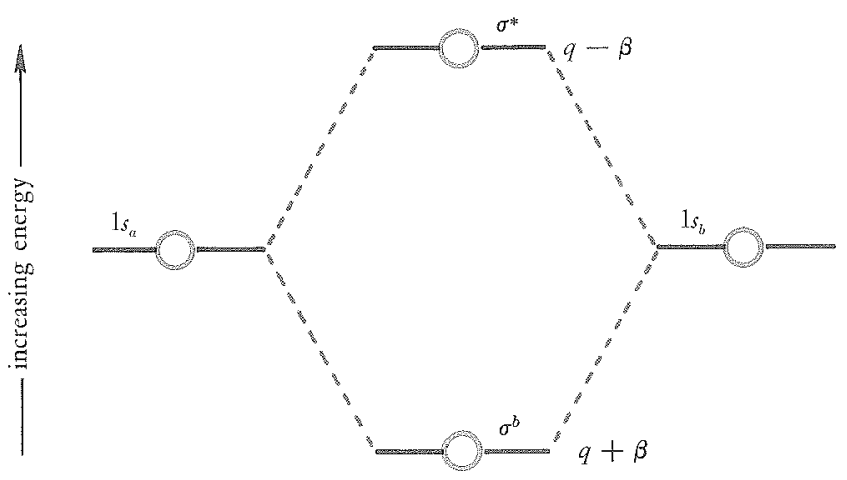

Figure 2-8 Relative molecularoorbital energies for $\mathrm{H}_{2}{ }^{+}$. 


\section{PROBLEM}

2-1. Calculate the energies of the $\sigma^{b}$ and $\sigma^{*}$ orbitals for $\mathrm{H}_{2}{ }^{+}$, including the overlap integral $S$. Show that $\sigma^{*}$ is destabilized more than $\sigma^{b}$ is stabilized if the overlap is different from zero.

\section{2-5 THE HYDROGEN MOLECULE}

The orbital electronic structures of molecules with more than one valence electron are built up by placing the valence electrons in the most stable molecular orbitals appropriate for the valence orbitals of the nuclei in the molecule. We have constructed the molecular orbitals for the system of two protons and two $1 s$ atomic orbitals. This set of orbitals is appropriate for $\mathrm{H}_{2}{ }^{+}, \mathrm{H}_{2}, \mathrm{H}_{2}{ }^{-}$, etc. The hydrogen molecule, $\mathrm{H}_{2}$, has two electrons that can be placed in the molecular orbitals given in the energy-level diagram (Fig. 2-8). Both electrons can be placed in the $\sigma^{b}$ level, provided they have different spin $\left(m_{s}\right)$ quantum numbers (the Pauli principle). Thus we represent the ground state of $\mathrm{H}_{2}$

ground state of $\mathrm{H}_{2}=\left(\sigma^{b}\right)^{2} \quad$ or $\quad\left[\sigma^{b}\left(m_{s}=+\frac{1}{2}\right)\right]\left[\sigma^{b}\left(m_{s}=-\frac{1}{2}\right)\right]$

which in our shorthand is $\left(\dot{\sigma}^{b}\right)\left(\bar{\sigma}^{b}\right)$.

This picture of the bond in $\mathrm{H}_{2}$ involving two electrons, each in a $\sigma^{b}$ orbital but with opposite spins, is analogous to the Lewis electronpair bond in $\mathrm{H}_{2}$ (Fig. 2-3). It is convenient to carry along the idea that a full bond between any two atoms involves two electrons. Thus we define as a useful theoretical quantity the number of bonds in a molecule as follows:

(number of electrons in bonding $\mathrm{MO}^{\prime} \mathrm{s}$ ) number of bonds $=\frac{\text { (number of electrons in antibonding MO's) }}{2}$

One electron in an antibonding $\mathrm{MO}$ is considered to cancel out the bonding stability imparted by one electron in a bonding MO. Using this formula we see that $\mathrm{H}_{2}+$ has half a $\sigma$ bond and $\mathrm{H}_{2}$ has one $\sigma$ bond. 


\section{2-6 BOND LENGTHS OF $\mathrm{H}_{2}{ }^{+}$AND $\mathrm{H}_{2}$}

A useful experimental quantity reflecting electronic structure is bond length. The standard bond length for a bond between any two atoms is the equilibrium internuclear separation. ${ }^{1}$ We shall express this distance between nuclei in Angstrom units and refer to it as $R$. The bond lengths of $\mathrm{H}_{2}{ }^{+}$and $\mathrm{H}_{2}$ in the ground state are 1.06 and $0.74 \mathrm{~A}$, respectively, as shown in Fig. 2-9. Thus the $\mathrm{H}_{2}$ molecule, with one $\sigma$ bond, has a shorter $R$ than does $\mathrm{H}_{2}{ }^{+}$, with only half a $\sigma$ bond. In general, when molecules with nuclei of approximately the same atomic number are compared, the bond length is shortest between the two atoms with the largest number of bonds.

\section{2-7 BOND ENERGIES OF $\mathrm{H}_{2}{ }^{+}$AND $\mathrm{H}_{2}$}

Another useful experimental quantity that reflects electronic structure is bond-dissociation energy. The standard bond-dissociation energy

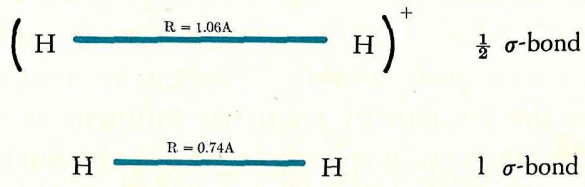

Figure 2-9 Comparison of $\mathrm{H}_{2}{ }^{+}$and $\mathrm{H}_{2}$.

${ }^{1}$ To make matters more complicated for us, nuclei in molecules are always vibrating. For example, the bond in $\mathrm{H}_{2}$, say, stretches and contracts as shown schematically below:

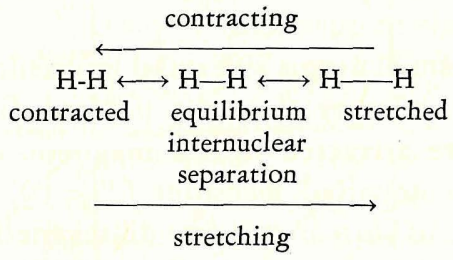

The equilibrium internuclear separation about which the nuclei vibrate is the standard bond length. 
for a bond between any two atoms is the energy required to break the bond, giving isolated ground-state atoms; i.e.,

$$
\mathrm{H}_{2}+\text { bond-dissociation energy } \rightarrow \mathrm{H}+\mathrm{H}
$$

We shall express bond energy in $\mathrm{kcal} /$ mole units, and refer to a particular bond energy as DE (atom 1-atom 2). The bond energies of $\mathrm{H}_{2}{ }^{+}$and $\mathrm{H}_{2}$ are 61.06 and $103.24 \mathrm{kcal} /$ mole, respectively. We see that $\mathrm{H}_{2}$, with one $\sigma$ bond, has a larger bond energy than $\mathrm{H}_{2}{ }^{+}$. This is again a very general result, since bond energies in an analogous series of molecules increase with an increasing number of bonds.

\section{2-8 PROPERTIES OF $\mathrm{H}_{2}{ }^{+}$AND $\mathrm{H}_{2}$ IN A MAGNETIC FIELD}

Most substances can be classified as either paramagnetic or diamagnetic according to their behavior in a magnetic field. A paramagnetic substance is attracted into a magnetic field with a force that is proportional to the product of the field strength and field gradient. A diamagnetic substance, on the other hand, is repelled by a magnetic field.

In general, atoms and molecules with unpaired electrons $(S \neq 0)$ are paramagnetic. Since electrons possess spin, an unpaired electron creates a permanent magnetic moment. There is in many cases a further contribution to the permanent magnetic moment as a result of the movement of the electron in its orbital about the nucleus (or nuclei, in the case of molecules). In addition to the permanent paramagnetic moment, magnetic moments are induced in atoms and molecules on the application of an external magnetic field. Such induced moments are opposite to the direction of the field; thus repulsion occurs. The magnitude of this repulsion is a measure of the diatnagnetism of the atom or molecule in question.

The paramagnetism of atoms and small molecules that results from unpaired electrons is larger than the induced diamagnetism; thus these substances are attracted into a magnetic field. Atoms and molecules with no unpaired electrons $(S=0)$, and therefore no paramagnetism due to electron spin, are diamagnetic and are repelled by a magnetic field.

The $\mathrm{H}_{2}+$ ion, with one unpaired electron $\left(S=\frac{1}{2}\right)$, is paramagnetic. 
The $\mathrm{H}_{2}$ molecule, with its two electrons paired $(S=0)$, is diamagnetic.

\section{2-9 SECOND-ROW HOMONUCLEAR DIATOMIC MOLECULES}

Let us proceed now to the atoms in the second row of the periodic table, namely, $\mathrm{Li}, \mathrm{Be}, \mathrm{B}, \mathrm{C}, \mathrm{N}, \mathrm{O}, \mathrm{F}$, and $\mathrm{Ne}$. These atoms have $2 s$, $2 p_{x}, 2 p_{y}$, and $2 p_{z}$ valence orbitals. We first need to specify a coordinate system for the general homonuclear diatomic molecule $A_{2}$, since the $2 p$ orbitals have directional properties. The $z$ axis is customarily assigned to be the unique molecular axis, as shown in Fig. 2-10. The molecular orbitals are obtained by adding and subtracting those atomic orbitals that overlap.

\section{$\sigma$ Orbitals}

The $2 s$ and $2 p_{z}$ orbitals combine to give $\sigma$ molecular orbitals, as illustrated in Fig. 2-11. The normalized wave functions are:

$$
\begin{aligned}
& \psi\left(\sigma_{s}^{b}\right)=\frac{1}{\sqrt{2}}\left(2 s_{a}+2 s_{b}\right) \\
& \psi\left(\sigma_{s}^{*}\right)=\frac{1}{\sqrt{2}}\left(2 s_{a}-2 s_{b}\right) \\
& \psi\left(\sigma_{z}^{b}\right)=\frac{1}{\sqrt{2}}\left(2 p_{z_{a}}+2 p_{z_{b}}\right)
\end{aligned}
$$

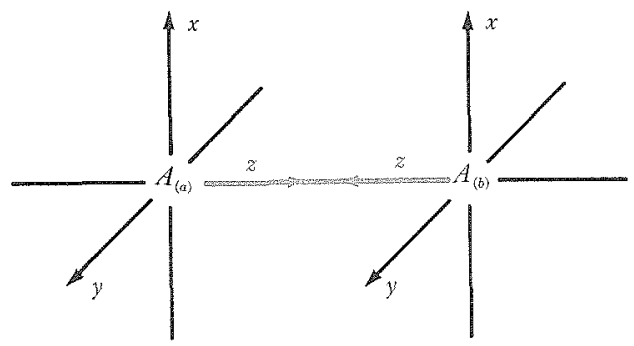

Figure 2-10 Coordinate system for an $\mathbf{A}_{2}$ molecule. 


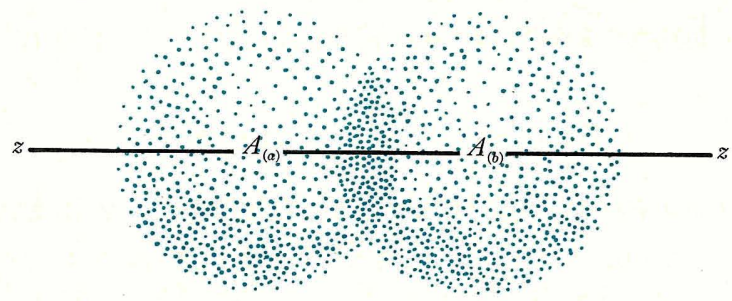

(a) overlap of $2 s$ valence orbitals

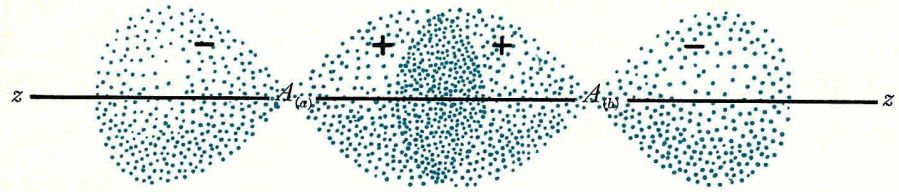

(b) overlap of $2 p_{z}$ valence orbitals

Figure 2-11 (a) Overlap of two $2 s$ valence orbitals in $\mathbf{A}_{2}$.

(b) Overlap of two $2 p_{z}$ valence orbitals in $\mathbf{A}_{2}$.

$$
\psi\left(\sigma_{z}^{*}\right)=\frac{1}{\sqrt{2}}\left(2 p_{z_{a}}-2 p_{z_{b}}\right)
$$

Notice that the $\sigma_{z}$ molecular orbitals are symmetric for rotation about the $z$ axis.

\section{$\pi$ Orbitals}

The $2 p_{x}$ and $2 p_{y}$ orbitals are not symmetric for rotation about the $z$ axis. The two $2 p_{x}$ orbitals overlap to give the molecular orbital shown in Fig. 2-12. This molecular orbital has a plus lobe on one side of the $z$ axis and a minus lobe on the other side. So if we rotate the molecular orbital by $180^{\circ}$, it simply changes sign. Multiplication by -1 restores the original orbital. In other words, there is a node in the $y z$ plane as shown in Fig. 2-13. A molecular orbital of this type is called a $\pi$ molecular orbital. It is clear that the two $2 p_{y}$ orbitals can also overlap to give $\pi$ molecular orbitals, which have a node in the $x z$ plane. There will be $\pi$ bonding $\left(\pi^{b}\right)$ and $\pi$ antibonding $\left(\pi^{*}\right)$ molecular orbitals; the more stable $\pi^{b}$ orbitals will have a 


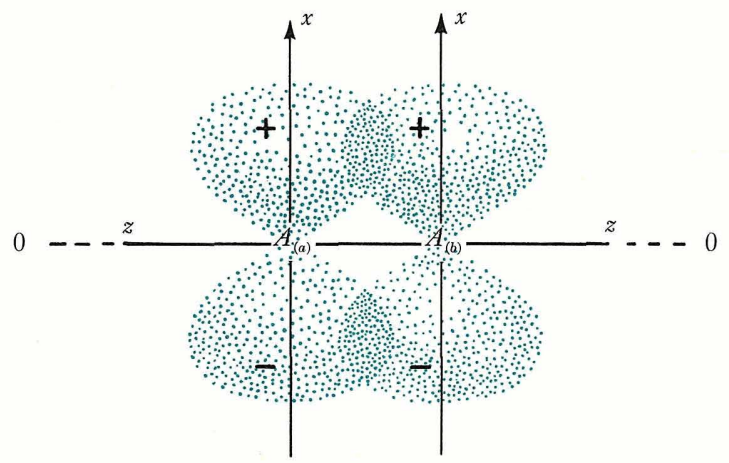

Figure 2-12 Overlap of two $2 p_{x}$ orbitals in $\mathbf{A}_{2}$.

concentration of electron density between the two A nuclei, whereas the less stable $\pi^{*}$ orbital will have a node between the two nuclei. Boundary surfaces of the $\sigma$ and $\pi$ molecular orbitals for $A_{2}$ molecules with $2 s$ and $2 p$ valence orbitals are shown in Fig. 2-14. The normalized wave functions for the $\pi$ MO's follow:

$$
\begin{aligned}
& \psi\left(\pi_{x}^{b}\right)=\frac{1}{\sqrt{2}}\left(2 p_{x_{a}}+2 p_{x_{b}}\right) \\
& \psi\left(\pi_{x}^{*}\right)=\frac{1}{\sqrt{2}}\left(2 p_{x_{a}}-2 p_{x_{b}}\right) \\
& \psi\left(\pi_{y}^{b}\right)=\frac{1}{\sqrt{2}}\left(2 p_{y_{a}}+2 p_{y_{b}}\right) \\
& \psi\left(\pi_{y}^{*}\right)=\frac{1}{\sqrt{2}}\left(2 p_{y_{a}}-2 p_{y_{b}}\right)
\end{aligned}
$$

The energy-level diagram for the molecular orbitals that accept the valence electrons can now be estimated. We know that the $2 s$ level is considerably more stable than $2 p$ in the atoms. The red line at $1.85 \mathrm{eV}$ in the emission spectrum of lithium is due to an electron falling from the $2 p$ to the more stable $2 s$ orbital. In fluorine, the $2 s-2 p$ energy difference is over $20 \mathrm{eV}$. Thus we place $2 p$ above $2 s$ in the energy-level diagram. ${ }^{1}$ Then the $\sigma^{b}, \sigma^{*}, \pi^{b}$, and $\pi^{*}$ orbitals are placed with the bonding levels more stable than the antibonding levels in

\footnotetext{
${ }^{1}$ See Appendix for neutral-atom orbital energies.
} 

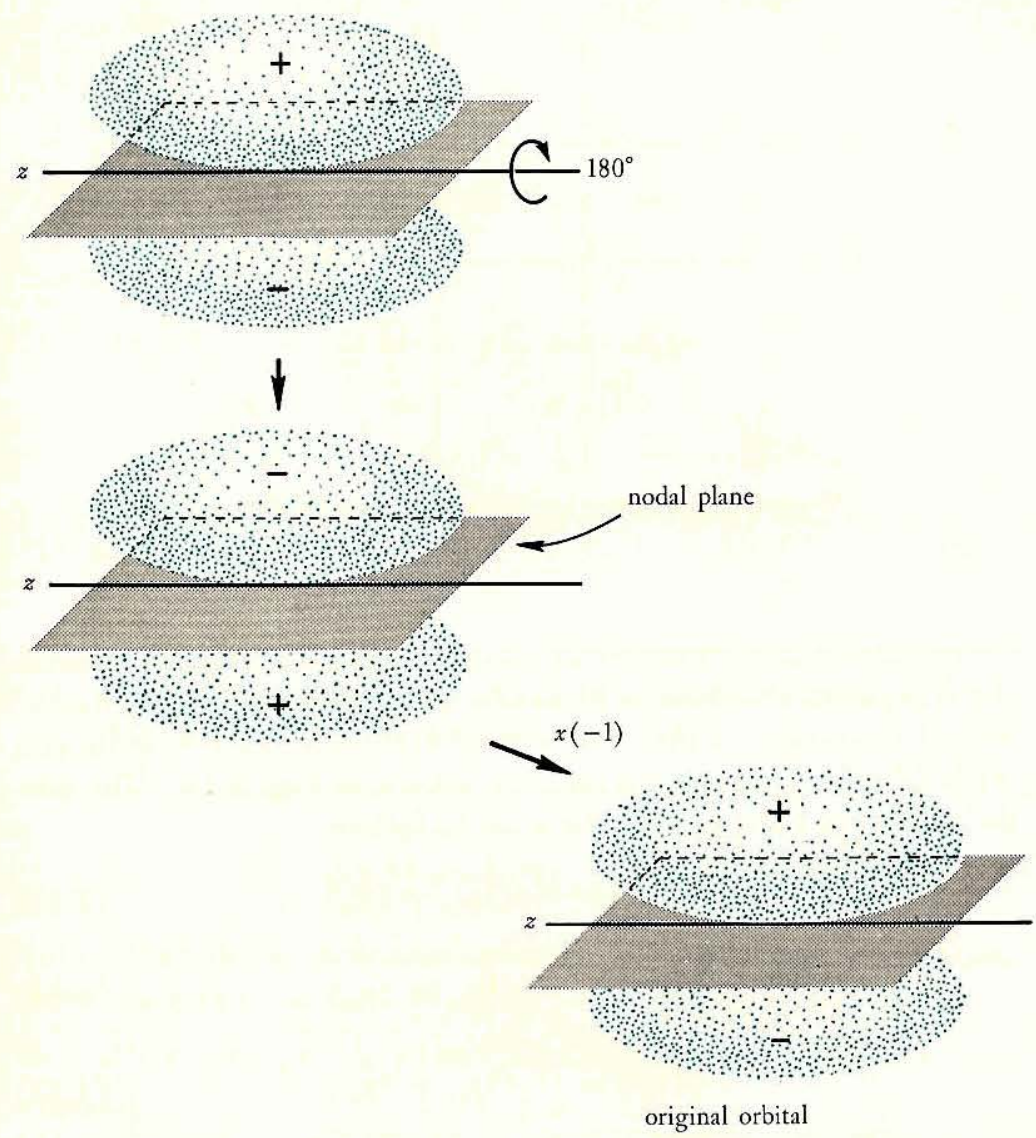

Figure 2-13 Rotation of a $\pi$ molecular orbital by $180^{\circ}$ about the internuclear axis.

any given combination. The possible energy-level diagrams are shown in Fig. 2-15.

The relative positioning of the $\sigma_{z}^{b}$ level is uncertain. When the $2 s-2 p$ energy difference is large, $\sigma_{z}^{b}$ is probably more stable than $\pi_{x, y^{b}}$, as shown in Fig. 2-15a. We should emphasize here that it is a good approximation to consider the $\sigma_{s}$ molecular orbitals as com- 

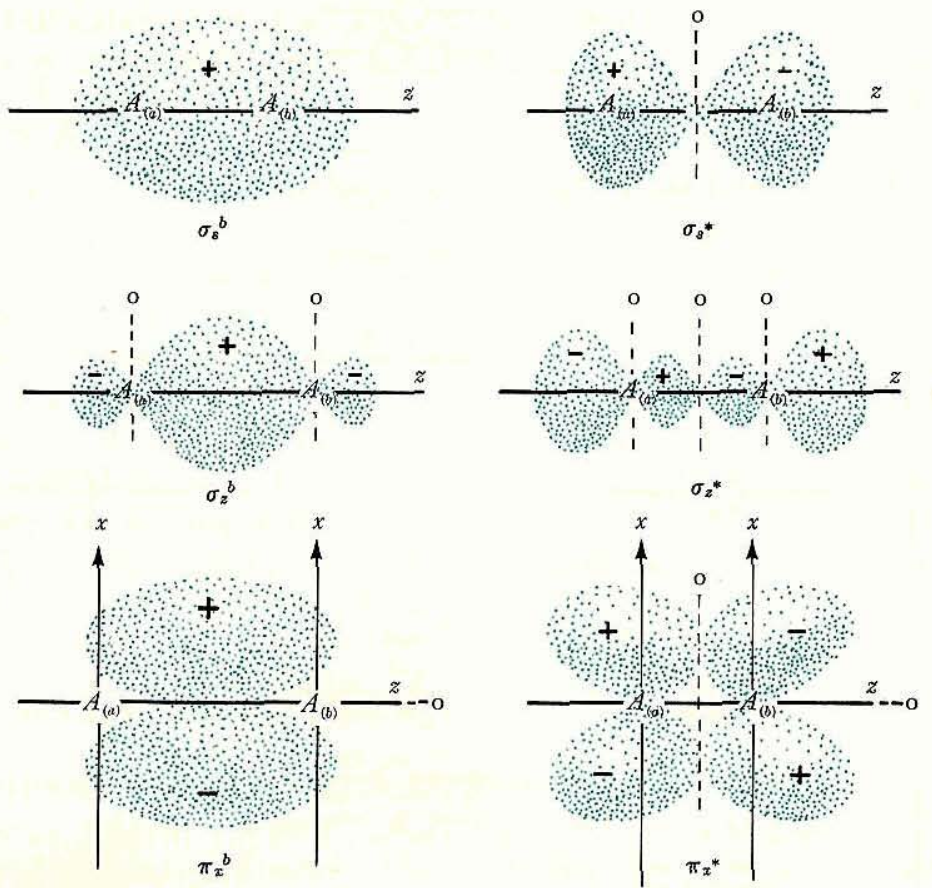

$$
\pi_{y}^{b} \text { and } \pi_{y}{ }^{*} \text { are equivalent to } \pi_{x}^{b} \text { and } \pi_{x}{ }^{*}
$$

Figure 2-14 Boundary surfaces of the $\sigma$ and $\pi$ molecular orbitals formed from $s$ and $p$ valence orbitals for a homonuclear diatomic molecule.

posed of the two $2 s$ atomic orbitals only if the $2 s-2 p$ energy difference is large. For small $2 s-2 p$ energy differences, we must consider the two $2 s$ and the two $2 p_{z}$ orbitals together in an LCAO-MO scheme. The most stable MO would be the combination

$$
\psi\left(\sigma_{s}^{b}\right)=\frac{1}{\sqrt{2\left(1+\tau^{2}\right)}}\left(2 s_{a}+\tau 2 p_{z_{a}}+2 s_{b}+\tau 2 p_{z_{b}}\right)
$$

where the coefficient $\tau$ is less than unity and represents the amount of $2 p$ included in the $\sigma_{s}^{b} \mathrm{MO}$. 


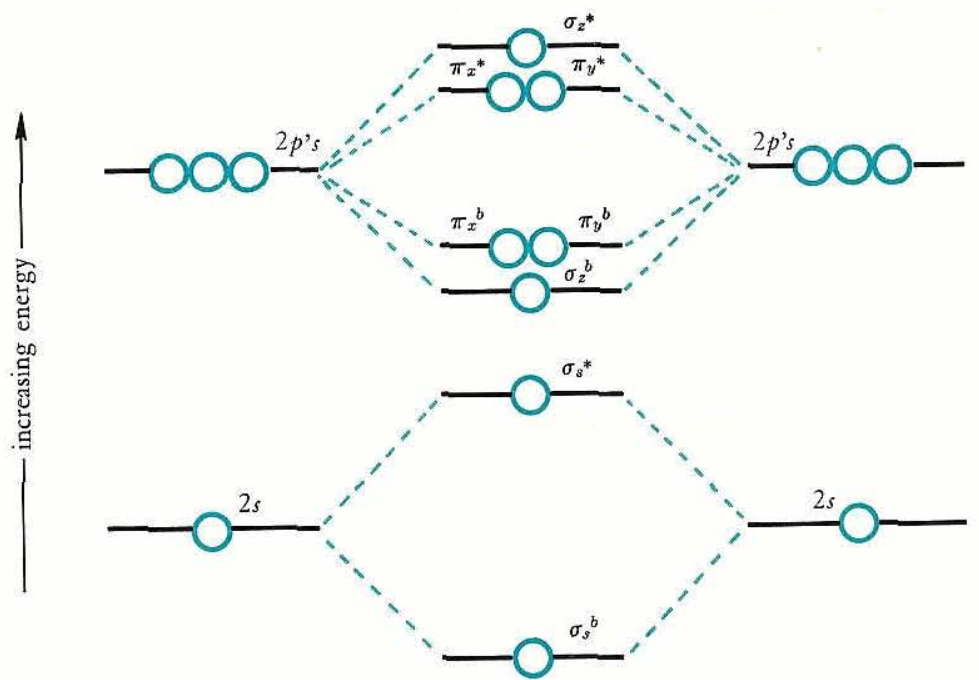

(a)

$A_{(a)}$ orbitals

$A_{2}$ orbitals

$A_{(b)}$ orbitals
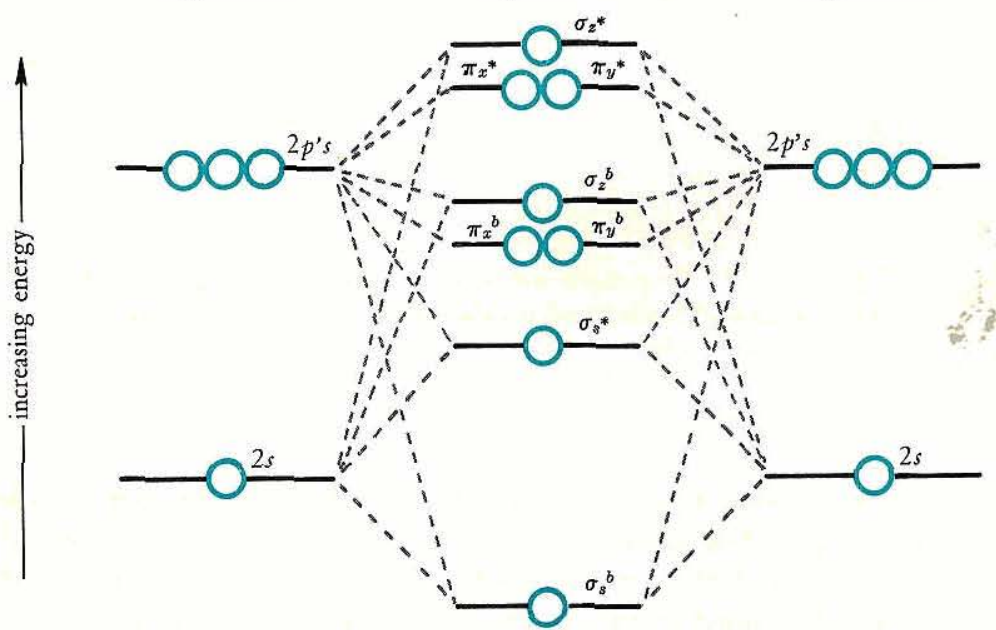

(b)

Figure 2-15 Molecular-orbital energy-level diagrams for a homonuclear diatomic molecule $(a)$ with no $\sigma_{s}-\sigma_{2}$ interaction; (b) with appreciable $\sigma_{s}-\sigma_{z}$ interaction. 
The stabilization of $\sigma_{s}^{b}$ and $\sigma_{s}{ }^{*}$ resulting from such $s-p$ bybridization is accompanied by a corresponding destabilization of $\sigma_{z}^{b}$ and $\sigma_{z}{ }^{*}$, these latter orbitals acquiring some $2 s$ character in the process. This effect is shown schematically in Fig. 2-16.

The final result for any reasonable amount of $s-p$ mixing is that the $\sigma_{z}{ }^{b}$ orbital becomes less stable than $\pi_{x, y^{b}}$, as shown in Fig. 2-15b. As we shall see in the pages to follow, the experimental information now available shows that the $\sigma_{z}{ }^{b}$ level is higher energy than the $\pi_{x},{ }^{b}$ level in most, if not all, diatomic molecules.

In Fig. $2-15$ the $\pi_{x}^{b}$ and $\pi_{y}^{b}$ levels are shown on the same line. There is no difference in overlap in the $\pi_{x}$ and $\pi_{y}$ molecular orbitals and thus they have the same energy, or, in the jargon of the profession, they are degenerate.

Using the molecular-orbital energy levels in Fig. 2-15, we shall discuss the electronic configurations of the second-row $\mathrm{A}_{2}$ molecules.

$L i_{2}$

The lithium atom has one $2 s$ valence electron. In $\mathrm{Li}$, the $2 s-2 p$ energy difference is small and the $\sigma_{s}{ }^{b} \mathrm{MO}$ of $\mathrm{Li}_{2}$ undoubtedly has considerable $2 p$ character. The two valence electrons in $\mathrm{Li}_{2}$ occupy the $\sigma_{s}^{b} \mathrm{MO}$, giving the ground-state configuration $\left(\sigma_{s}^{b}\right)^{2}$. Consistent with the theory, experimental measurements show that the lithium

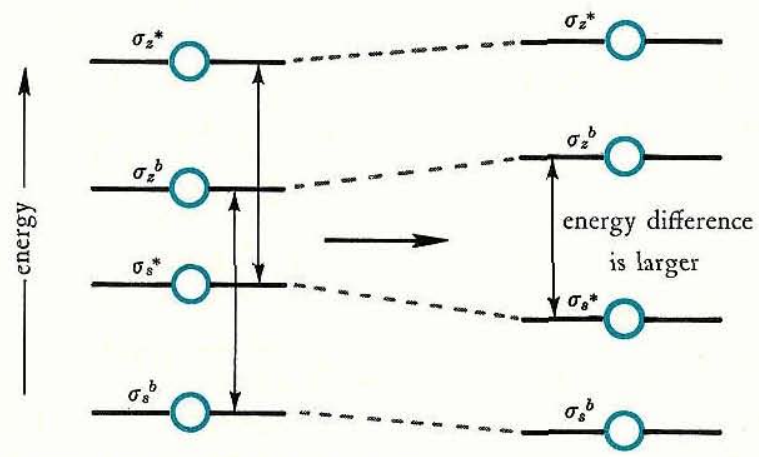

Figure 2-16 Schematic drawing of the effect of $\sigma_{s}-\sigma_{z}$ interaction on the energies of $\sigma_{s}^{b}, \sigma_{s}{ }^{*}, \sigma_{z}^{b}$, and $\sigma_{z}{ }^{*}$. 
molecule has no unpaired electrons. With two electrons in a bonding $\mathrm{MO}$, there is one net bond. The bond length of $L i_{2}$ is $2.67 \mathrm{~A}$ as compared with $0.74 \mathrm{~A}$ for $\mathrm{H}_{2}$. The larger $R$ for $\mathrm{Li}_{2}$ is partially due to the shielding of the two $\sigma_{s}^{b}$ valence electrons by the electrons in the inner $1_{s}$ orbitals. This shielding reduces the attractions of the nuclei and the electrons in the $\sigma_{s}^{b} \mathrm{MO}$. The mutual tepulsion of the two $1 s$ electron pairs, an interaction not present in $\mathrm{H}_{2}$, is also partly responsible for the large $R$ of $\mathrm{Li}_{2}$. The bond energies of $\mathrm{H}_{2}$ and $\mathrm{Li}_{2}$ are 103 and $25 \mathrm{kcal} /$ mole, respectively. The smaller bond energy of $\mathrm{Li}_{2}$ is again undoubtedly due to the presence of the two $1 s$ electron pairs, as discussed above.

$\mathrm{Be}_{2}$

The beryllium atom has the valence electronic structure $2 s^{2}$. The electronic configuration of $\mathrm{Be}_{2}$ would be $\left(\sigma_{s}^{b}\right)^{2}\left(\sigma_{s}^{*}\right)^{2}$. This configuration gives no net bonds $[(2-2) / 2=0]$ and thus is consistent with the absence of $\mathrm{Be}_{2}$ from the family of $\mathrm{A}_{2}$ molecules.

$B_{2}$

Boron is $2 s^{2} 2 p^{1}$. The electronic configuration of $\mathrm{B}_{2}$ depends on the relative positioning of the $\sigma_{z}{ }^{b}$ and the $\pi_{x, y}{ }^{b}$ levels. Experimental measurements indicate that the boron molecule has two unpaired electrons in the $\pi_{x, y^{b}}$ level. Thus the electronic configuration of $\mathrm{B}_{2}$ is $\left(\sigma_{s}^{b}\right)^{2}\left(\sigma_{s}{ }^{*}\right)^{2}\left(\pi_{x}^{b}\right)\left(\pi_{y}^{b}\right)$, giving one net $\pi$ bond. The bond length of $B_{2}$ is $1.59 \mathrm{~A}$. The bond energy of $B_{2}$ is $69 \mathrm{kcal} /$ mole.

$C_{2}$

Carbon is $2 s^{2} 2 p^{2}$. In carbon the $\sigma_{z}{ }^{b}$ and $\pi_{x, y^{b}}$ levels are so spaced that both the $\left(\sigma_{s}^{b}\right)^{2}\left(\sigma_{s}^{*}\right)^{2}\left(\pi_{x, y^{b}}\right)^{4}$ and the $\left(\sigma_{s}^{b}\right)^{2}\left(\sigma_{s}^{*}\right)^{2}\left(\pi_{x, y^{b}}\right)^{3}\left(\sigma_{z}^{b}\right)$ configurations have approximately the same energy. The latest view is that the configuration $\left(\sigma_{s}^{b}\right)^{2}\left(\sigma_{s}^{*}\right)^{2}\left(\pi_{x, y}^{b}\right)^{4}$ is the ground state (by less than $0.1 \mathrm{eV})$. In this state there are no unpaired electrons and a total of two $\pi$ bonds. This means that $\sigma_{z}^{b}$ must be considerably higher energy than $\pi_{x},{ }^{b}$ in $\mathrm{C}_{2}$, since the lowest state in the $\left(\sigma_{s}^{b}\right)^{2}\left(\sigma_{s}{ }^{*}\right)^{2}$ $\left(\pi_{x}, y^{b}\right)^{3}\left(\sigma_{z}^{b}\right)$ configuration has two unpaired electrons. Electron pairing requires energy (recall Hund's first rule). The two bonds predicted for $C_{2}$ may be compared with the experimentally observed bond energy of $150 \mathrm{kcal} / \mathrm{mole}$ and the bond length of $1.31 \mathrm{~A}$. 
$\mathrm{N}_{2}$

Nitrogen is $2 s^{2} 2 p^{3}$. The electronic configuration of $\mathrm{N}_{2}$ is $\left(\sigma_{s}^{b}\right)^{2}$ $\left(\sigma_{s}{ }^{*}\right)^{2}\left(\pi_{x, y^{b}}\right)^{4}\left(\sigma_{z}^{b}\right)^{2}$, consistent with the observed diamagnetism of this molecule. The nitrogen molecule has three net bonds (one $\sigma$ and two $\pi$ ), the maximum for an $A_{2}$ molecule, thus accounting for its unusual stability, its extraordinarily large bond energy of $225 \mathrm{kcal} /$ mole, and its very short $R$ of $1.10 \mathrm{~A}$.

We wish to emphasize here that the bighest filled orbital in $\mathrm{N}_{2}$ is $\sigma_{z}^{b}$, which is contrary to the popular belief that $\pi_{x, y}{ }^{b}$ is the bigher level. The experimental evidence comes from a detailed analysis of the electronic spectrum of $\mathrm{N}_{2}$, and from spectroscopic and magnetic experiments that establish that the most stable state for $\mathrm{N}_{2}+$ arises from the configuration $\left(\sigma_{s}^{b}\right)^{2}\left(\sigma_{s}^{*}\right)^{2}\left(\pi_{x}, y^{b}\right)^{4}\left(\sigma_{z}^{b}\right)$.

$\mathrm{O}_{2}$

Oxygen is $2 s^{2} 2 p^{4}$. The electronic configuration of $\mathrm{O}_{2}$ is $\left(\sigma_{s}^{b}\right)^{2}$ $\left(\sigma_{s}{ }^{*}\right)^{2}\left(\sigma_{z}{ }^{b}\right)^{2}\left(\pi_{x, y^{b}}\right)^{4}\left(\pi_{x}{ }^{*}\right)\left(\pi_{y}{ }^{*}\right)$. The electrons in $\pi_{x, y^{*}}$ have the same spin in the ground state, resulting in a prediction of two unpaired electrons in $\mathrm{O}_{2}$; the oxygen molecule is paramagnetic to the extent of two unpaired spins in agreement with theory. The explanation of the paramagnetism of $\mathrm{O}_{2}$ gave added impetus to the use of the molecular-orbital theory, since from the simple Lewis picture it is not at all clear why $\mathrm{O}_{2}$ should have two unpaired electrons.

Two net bonds (one $\sigma$, one $\pi$ ) are predicted for $\mathrm{O}_{2}$. The bond energy of $\mathrm{O}_{2}$ is $118 \mathrm{kcal} / \mathrm{mole}$, and $R=1.21 \mathrm{~A}$. The change in bond length on changing the number of electrons in the $\pi_{x, y} *$ level of the $\mathrm{O}_{2}$ system is very instructive. The accurate bond length of $\mathrm{O}_{2}$ is $1.2074 \mathrm{~A}$. When an electron is removed from $\pi_{x, y^{*}}$, giving $\mathrm{O}_{2}{ }^{+}$, the bond length decreases to $1.1227 \mathrm{~A}$. Formally, the number of bonds has increased from 2 to $2 \frac{1}{2}$. When an electron is added to the $\pi_{x, y}{ }^{*}$ level of $\mathrm{O}_{2}$, giving $\mathrm{O}_{2}^{-}$, the bond length increases to $1.26 \mathrm{~A}$; addition of a second electron to give $\mathrm{O}_{2}{ }^{2-}$ increases the bond length still further to $1.49 \mathrm{~A}$. This is in agreement with the prediction of $1 \frac{1}{2}$ bonds for $\mathrm{O}_{2}^{-}$and 1 bond for $\mathrm{O}_{2}^{2-}$.

$F_{2}$

Fluorine is $2 s^{2} 2 p^{5}$. The electronic configuration of $\mathrm{F}_{2}$ is $\left(\sigma_{s}^{b}\right)^{2}$ $\left(\sigma_{s}^{*}\right)^{2}\left(\sigma_{s}^{b}\right)^{2}\left(\pi_{x, y^{b}}\right)^{4}\left(\pi_{x, y^{*}}\right)^{4}$, leaving no unpaired electrons and one net 
bond. This electronic structure is consistent with the diamagnetism of $F_{2}$, the $36-\mathrm{kcal} /$ mole $F-F$ bond energy, and the $R$ of $1.42 \mathrm{~A}$.

$\dot{N} e_{2}$

Neon has a closed-shell electronic configuration $2 s^{2} 2 p^{6}$. The hypothetical $\mathrm{Ne}_{2}$ would have the configuration $\left(\sigma_{s}^{b}\right)^{2}\left(\sigma_{s}^{*}\right)^{2}\left(\sigma_{z}^{b}\right)^{2}\left(\pi_{x, y^{b}}\right)^{4}$ $\left(\pi_{x, y^{*}}\right)^{4}\left(\sigma_{z}{ }^{*}\right)^{2}$ and zero net bonds. To date there is no experimental evidence for the existence of a stable neon molecule.

\section{2-10 OTHER A MOLECULES}

With proper adjustment of the $n$ quantum number of the valence orbitals, the MO energy-level diagrams shown in Fig. 2-15 for second-row $A_{2}$ molecules can be used to describe the electronic structures of $A_{2}$ molecules in general.

$N a_{2}, K_{2}, R b_{2}, C s_{2}$

The alkali metal diatomic molecules all have the ground-state configuration $\left(\sigma_{s}^{b}\right)^{2}$, with one $\sigma$ bond. They are diamagnetic. The bond lengths and bond energies of $\mathrm{Li}_{2}, \mathrm{Na}_{2}, \mathrm{~K}_{2}, \mathrm{Rb}_{2}$, and $\mathrm{Cs}_{2}$ are given in Table 2-1.. The bond lengths increase and the bond energies de-

Table 2-1

Bond Lengths and Bond Energies of Alkali Metal Molecules ${ }^{\mathrm{a}}$

\begin{tabular}{ccc}
\hline Molecule & Bond length, A & $\begin{array}{c}\text { Bond energy, } \\
\text { kcal/mole }\end{array}$ \\
\hline $\mathrm{Li}_{2}$ & 2.672 & 25 \\
$\mathrm{Na}_{2}$ & 3.078 & 17.3 \\
$\mathrm{~K}_{2}$ & 3.923 & 11.8 \\
$\mathrm{Rb}_{2}$ & & 10.8 \\
$\mathrm{Cs}_{2}$ & 10.4 \\
\hline
\end{tabular}

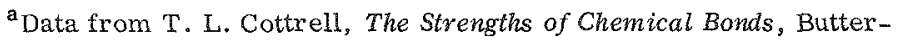
worths, London, 1958, Table 11.5.1. 
crease, regularly, from $\mathrm{Li}_{2}$ to $\mathrm{Cs}_{2}$. These effects presumably are due to the increased shielding of the $\sigma_{s}^{b}$ electrons by inner-shell electrons in going from $\mathrm{Li}_{2}$ to $\mathrm{Cs}_{2}$.

$\mathrm{Cl}_{2}, \mathrm{Br}_{2}, \mathrm{I}_{2}$

The ground-state electronic configuration of the halogen molecules is $\left(\sigma_{s}^{b}\right)^{2}\left(\sigma_{s}^{*}\right)^{2}\left(\sigma_{z}^{b}\right)^{2}\left(\pi_{x, y^{b}}\right)^{4}\left(\pi_{x, y}\right)^{4}$, indicating one net $\sigma$ bond. The molecules are diamagnetic. Table $2-2$ gives bond lengths and bond energies for $\mathrm{F}_{2}, \mathrm{Cl}_{2}, \mathrm{Br}_{2}$, and $\mathrm{I}_{2}$. The bond lengths increase predictably from $F_{2}$ to $I_{2}$, but the bond energies are irregular, increasing from $\mathrm{F}_{2}$ to $\mathrm{Cl}_{2}$ and then decreasing from $\mathrm{Cl}_{2}$ to $\mathrm{I}_{2}$. The fact that the bond energy of $\mathrm{Cl}_{2}$ is larger than that of $\mathrm{F}_{2}$ is believed to be due to the smaller repulsions of electron pairs in the $\pi$ orbitals of $\mathrm{Cl}_{2}$. One explanation which has been advanced is that the reduced repulsions follow from the interaction of the empty chlorine $3 d$ orbitals in the $\pi$ MO system. As a result of such $p_{\pi}-d_{\pi}$ interaction, the electron pairs in $\mathrm{Cl}_{2}$ have a greater chance to avoid each other. However, it is not necessary to use the $p_{\pi}-d_{\pi}$ explanation, since we know from atomic spectra that the interelectronic repulsions in the $2 p$ orbitals of $\mathrm{F}$ are considerably larger than the repulsions in the $3 p$ orbitals of $\mathrm{Cl}$.

Table $2-2$

Bond Lengths and Bond Energies of Halogen Molecules ${ }^{a}$

\begin{tabular}{ccc}
\hline Molecule & Bond length, $A$ & $\begin{array}{c}\text { Bond energy, } \\
\text { kcal/mole }\end{array}$ \\
\hline $\mathrm{F}_{2}$ & 1.418 & 36 \\
$\mathrm{Cl}_{2}$ & 1.988 & 57.07 \\
$\mathrm{Br}_{2}$ & 2.283 & 45.46 \\
$\mathrm{I}_{2}$ & 2.667 & 35.55 \\
\hline
\end{tabular}

${ }^{a}$ Data from T. L. Cottrell, The Strengths of Chemical Bonds, Butterworths, London, 1958, Table 11.5.1. 
Table 2-3

Quantum Number Assignments for Molecular Orbitals in Linear Molecules

\begin{tabular}{crl}
\hline Molecular orbitals & $m_{l}$ & Atomic orbitals \\
\hline$\sigma$ & 0 & $s, p_{z}, d_{z^{2}}$ \\
$\pi$ & \pm 1 & $p_{x}, p_{y}, d_{x z}, d_{y z}$ \\
0 & \pm 2 & $d_{x y}, d_{x^{2}-y^{2}}$ \\
\hline
\end{tabular}

\section{2-11 TERM SYMBOLS FOR LINEAR MOLECULES}

Electronic states of a linear molecule may be classified conveniently in terms of angular momentum and spin, analogous to the RussellSaunders term-symbol scheme for atoms. The unique molecular axis in linear molecules is labeled the $z$ axis. The combining atomic orbitals in any given molecular orbital have the same $m_{l}$ value. Thus an $m_{l}$ quantum number is assigned to each different type of $\mathrm{MO}$, as indicated in Table 2-3. The term designations are of the form

$$
{ }^{2 S+1}\left|\dot{M}_{L}\right|
$$

where $S$ has the same significance as for atoms. The $M_{L}$-state abbreviations are given in Table $2-4$.

We shall work two examples in order to illustrate the procedure.

Table $2-4$

State Symbols Corresponding to $M_{L}$ Values in Linear-Molecule Electronic-State Classification

\begin{tabular}{cc}
\hline State & $M_{L}$ \\
\hline$\Sigma$ & 0 \\
$\Pi$ & \pm 1 \\
$\Delta$ & \pm 2 \\
$\Phi$ & \pm 3 \\
\hline
\end{tabular}




\section{EXAMPLE 2-1}

The ground-state term of $\mathrm{H}_{2}$ is found as follows.

1. Find $M_{L}$ : The two electrons are placed in the $\sigma^{b} \mathrm{MO}$ shown in Fig. 2-8, giving the $\left(\sigma^{b}\right)^{2}$ configuration. This is the most stable state of $\mathrm{H}_{2}$. The $\mathrm{MO}$ is $\sigma$ type, so each electron has $m_{l}=0$. Then

$$
M_{L_{s}}=m_{l_{1}}+m_{l_{2}}=0+0=0
$$

and the state is 2 .

2. Find $M_{S}$ : Since both electrons have $m_{l}=0$, they must have different $m_{s}$ values (the Pauli principle). Thus,

$$
M_{S}=m_{s_{1}}+m_{s_{2}}=\left(+\frac{1}{2}\right)+\left(-\frac{1}{2}\right)=0
$$

with $M_{S}=0, S=0$. The correct term symbol ${ }^{1}$ is therefore ${ }^{1} \Sigma$.

From the result in the $\mathrm{H}_{2}$ case, you may suspect that filled molecular orbitals always give $M_{L}=0$ and $M_{S}=0$. Indeed this is so, since in filled orbitals every positive $m_{l}$ value is matched with a canceling negative $m_{l}$ value. The same is true for the $m_{s}$ values; they come in $+\frac{1}{2},-\frac{1}{2}$ paits in filled orbitals. This information eliminates considerable work in arriving at the term symbols for states of molecules in which there are many electrons, since most of the electrons are paired in different molecular orbitals.

\section{EXAMPLE 2-2}

Let us now find the ground-state term for $\mathrm{O}_{2}$. The electronic configuration of $\mathrm{O}_{2}$ is $\left(\sigma_{s}^{b}\right)^{2}\left(\sigma_{s}^{*}\right)^{2}\left(\sigma_{z}^{b}\right)^{2}\left(\pi_{x, y^{b}}\right)^{4}\left(\pi_{x, y^{*}}\right)^{2}$. All the orbitals are filled and give $M_{L}=0$ up to $\pi_{x, y}{ }^{*}$. The two electrons in $\pi^{*}$ can be arranged as shown in Table $2-5$.

There is a term with $M_{L}=+2,-2$, and $M_{S}=0(S=0)$; the term designation is ${ }^{1} \Delta$. There is a term with $M_{L}=0$ and $M_{S}=$ $+1,0,-1(S=1)$; the term designation is ${ }^{3} \Sigma$. This leaves one microstate unaccounted for, with $M_{L}=0$ and $M_{S}=O(S=0)$; thus there is a ${ }^{1} \Sigma$ term.

The ground state must be either ${ }^{1} \Delta,{ }^{3} \Sigma$, or ${ }^{1} \Sigma$. According to

${ }^{1}$ There are additional designations possible in certain linear molecules, depending on the symmetry properties of the molecular wave function. For example, the complete symbol for the ground state of $\mathrm{H}_{2}$ is ${ }^{1} \Sigma_{g}{ }^{+}$. A discussion of the complete notation is given in C. J. Ballhausen and H. B. Gray, Molecular Orbital Tbeory, Benjamin, New York, 1964, Chap. 3. 
Table 2-5

$M_{L}, M_{S}$ Values for Example 2-2

\begin{tabular}{|c|c|c|c|}
\hline \multirow{2}{*}{$M_{L}$} & \multicolumn{3}{|c|}{$M_{S}$} \\
\hline & 1 & 0 & -1 \\
\hline 2 & & $\left(\pi_{1}^{+} \bar{\pi}_{1}\right)$ & \\
\hline 1 & & & \\
\hline 0 & $\left(\pi_{1}^{+} \bar{\pi}_{-1}\right)$ & $\begin{array}{l}\left(\bar{\pi}_{1} \bar{\pi}_{-1}\right) \\
\left(\bar{\pi}_{1}^{+} \bar{\pi}_{-1}\right)\end{array}$ & $\left(\bar{\pi}_{1} \bar{\pi}_{-1}\right)$ \\
\hline-1 & & & \\
\hline-2 & & $\left(\pi_{-1} \bar{\pi}_{-1}\right)$ & \\
\hline
\end{tabular}

Hund's first rule the ground state has the highest spin multiplicity; the ground state is therefore ${ }^{3} \Sigma$. As we discussed earlier, the ${ }^{3} \Sigma$ ground state predicted by the molecular-orbital theory is consistent with the experimental results, since $\mathrm{O}_{2}$ is paramagnetic to the extent of two unpaired electrons $(S=1)$. Spectroscopic evidence also confirms the ${ }^{3} \Sigma$ ground state for $\mathrm{O}_{2}$.

In Table 2-6 are listed the ground-state terms and other pertinent information for several homonuclear diatomic molecules.

\section{2-12 HETERONUCLEAR DiATOMiC MOLECULES}

Two different atoms are bonded together in a beteronuclear diatomic molecule. A simple example for a discussion of bonding is lithium hydride, $\mathrm{LiH}$.

The valence orbitals of $\mathrm{Li}$ are $2 s, 2 p_{x}, 2 p_{y}$, and $2 p_{z}$. The valence orbital of $\mathrm{H}$ is $1 s$. Fig. 2-17 shows the overlap of the hydrogen $1 s$ orbital with the $2 s, 2 p_{x}, 2 p_{y}$, and $2 p_{z}$ lithium orbitals. The first step is to classify the valence orbitals as $\sigma$ or $\pi$ types. The $1 s$ of $\mathrm{H}$ and the $2 s$ and $2 p_{z}$ of Li are $\sigma$ valence orbitals. Thus, the lithium $2 s$ and 
$2 p_{z}$ orbitals can be combined with the $1 s$ orbital of hydrogen. The $2 p_{x}$ and $2 p_{y}$ orbitals of $\mathrm{Li}$ are $\pi$ valence orbitals and do not interact with the $\sigma$ type $1 s$ orbital of $\mathrm{H}$. The overlap of $2 p_{x}$ (or $2 p_{y}$ ) with $1 s$ is zero, as shown in Fig. 2-17.)

We shall now discuss the $\sigma$-molecular-orbital system in some detail. Since the $2 s$ level of $\mathrm{Li}$ is more stable than the $2 p$ level, it is a good approximation to consider the $\sigma^{b}$ molecular orbital as composed mainly of the hydrogen $1 s$ and the lithium $2 s$ orbitals.

It is also important to note that the $1 s$ orbital of $\mathrm{H}$ is much more stable than the $2 s$ orbital of $\mathrm{Li}$. We know that in the free atoms this stability difference is large, since the first ionization potential of $\mathrm{Li}\left(1 s^{2} 2 s \rightarrow 1 s^{2}\right)$ is $5.4 \mathrm{eV}$ and the ionization potential of $\mathrm{H}$ is $13.6 \mathrm{eV}$. As a consequence of the greater stability of the hydrogen $1 s$ orbital, an electron in the $\sigma^{b}$ molecular orbital spends most of its time in the vicinity of the $\mathrm{H}$ nucleus.
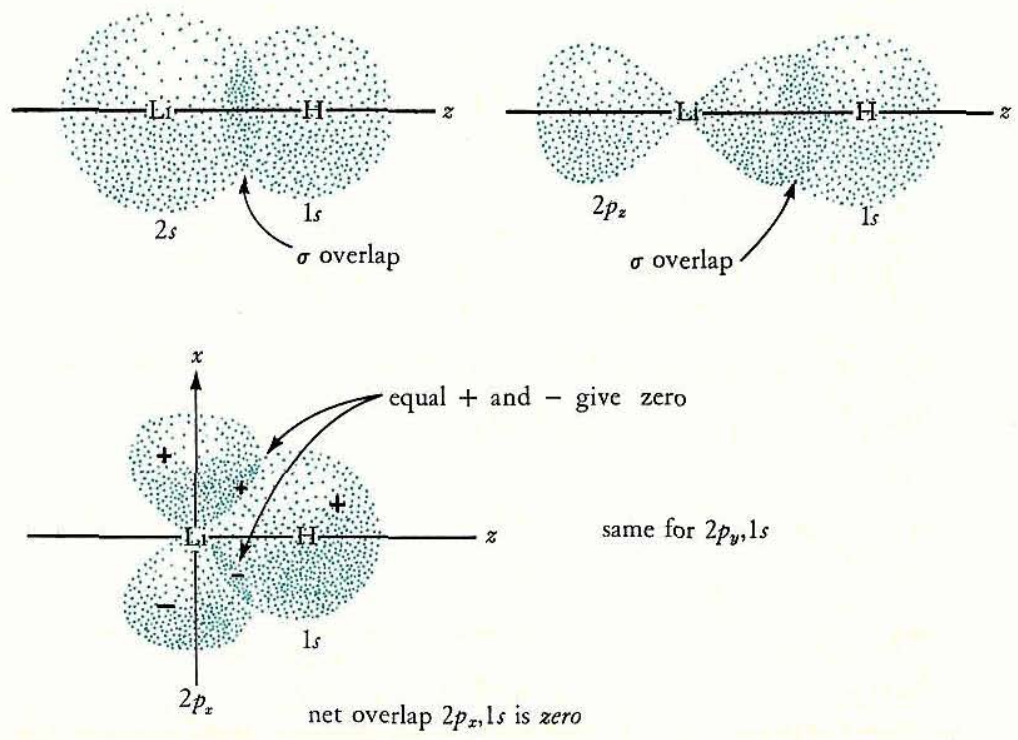

Figure 2-17 Overlap of the hydrogen 1s orbital with the lithium valence orbitals. 
Table $2-6$

Properties of Homonuclear Diatomic Molecules ${ }^{a}$

\begin{tabular}{|c|c|c|c|}
\hline Molecule & $\begin{array}{l}\text { Ground } \\
\text { state }\end{array}$ & $\begin{array}{l}\text { Bond } \\
\text { length, } A\end{array}$ & $\begin{array}{l}\text { Bond-dissociation } \\
\text { energy, kcal/mole }\end{array}$ \\
\hline $\mathrm{Ag}_{2}$ & ${ }^{1} \sum ?$ & & 39 \\
\hline $\mathrm{As}_{2}$ & ${ }^{1} \Sigma$ & & 91 \\
\hline $\mathrm{Au}_{2}$ & ${ }^{1} \sum ?$ & & 52 \\
\hline $\mathrm{B}_{2}$ & ${ }^{3} \Sigma$ & 1.589 & 69 \\
\hline $\mathrm{Bi}_{2}$ & ${ }^{1} \Sigma$ & & 39.2 \\
\hline $\mathrm{Br}_{2}$ & ${ }^{1} \Sigma$ & 2.283 & 45.46 \\
\hline $\mathrm{C}_{2}$ & ${ }^{1} \sum\left({ }^{3} \Pi\right)^{b}$ & 1.3117 & 150 \\
\hline $\mathrm{Cd}_{2}$ & ${ }^{1} \sum ?$ & & 2.1 \\
\hline $\mathrm{Cl}_{2}$ & ${ }^{1} \Sigma$ & 1.988 & 57.07 \\
\hline $\mathrm{Cl}_{2}{ }^{+}$ & ${ }^{2} \mathrm{II}$ & 1.891 & \\
\hline $\mathrm{Cs}_{2}$ & ${ }^{1} \Sigma$ & & 10.4 \\
\hline $\mathrm{Cu}_{2}$ & ${ }^{1} \sum$ & & 47 \\
\hline $\mathrm{D}_{2}$ & ${ }^{1} \sum$ & 0.7416 & \\
\hline$F_{2}$ & ${ }^{1} \sum$ & 1.418 & 36 \\
\hline $\mathrm{Ga}_{2}$ & & & 35 \\
\hline $\mathrm{Ge}_{2}$ & & & 65 \\
\hline $\mathrm{H}_{2}$ & ${ }^{1} \sum$ & 0.7415 & 103.24 \\
\hline $\mathrm{H}_{2}{ }^{+}$ & ${ }^{2} \sum$ & 1.06 & 61.06 \\
\hline $\mathrm{He}_{2}^{+}$ & ${ }^{2} \sum$ & 1.08 & \\
\hline $\mathrm{Hg}_{2}$ & ${ }^{1} \sum$ & & 3.2 \\
\hline $\mathrm{I}_{2}$ & ${ }^{1} \sum$ & 2.6666 & 35.55 \\
\hline $\mathrm{K}_{2}$ & ${ }^{1} \Sigma$ & 3.923 & 11.8 \\
\hline $\mathrm{Li}_{2}$ & ${ }^{1} \sum$ & 2.672 & 25 \\
\hline $\mathrm{N}_{2}$ & ${ }^{1} \Sigma$ & 1.0976 & 225.0 \\
\hline
\end{tabular}


Table 2-6 (continued)

\begin{tabular}{|c|c|c|c|}
\hline Molecule & $\begin{array}{l}\text { Ground } \\
\text { state }\end{array}$ & $\begin{array}{l}\text { Bond } \\
\text { length, A }\end{array}$ & $\begin{array}{l}\text { Bond-dissociation } \\
\text { energy, kcal/mole }\end{array}$ \\
\hline $\mathrm{N}_{2}{ }^{+}$ & ${ }^{2} \sum$ & 1.116 & \\
\hline $\mathrm{Na}_{2}$ & ${ }^{1} \sum$ & 3.078 & 17.3 \\
\hline $\mathrm{O}_{2}$ & ${ }^{3} \Sigma$ & 1.20741 & 117.96 \\
\hline $\mathrm{O}_{2}^{+}$ & ${ }^{2} \Pi$ & 1.1227 & \\
\hline $\mathrm{O}_{2}^{-}$ & ${ }^{2} \Pi ?$ & 1.26 & \\
\hline $\mathrm{O}_{2}{ }^{2-}$ & ${ }^{1} \sum ?$ & 1.49 & \\
\hline $\mathrm{P}_{2}$ & ${ }^{1} \Sigma$ & 1.8943 & 116.0 \\
\hline $\mathrm{Pb}_{2}$ & & & 23 \\
\hline $\mathrm{Rb}_{2}$ & ${ }^{1} \Sigma$ & & 10.8 \\
\hline $\mathrm{S}_{2}$ & ${ }^{3} \Sigma$ & 1.887 & 83 \\
\hline $\mathrm{Sb}_{2}$ & ${ }^{1} \Sigma$ & & 69 \\
\hline $\mathrm{Se}_{2}$ & ${ }^{3} \sum$ & 2.152 & 65 \\
\hline $\mathrm{Si}_{2}$ & & 2.252 & 75 \\
\hline $\mathrm{Sn}_{2}$ & & & 46 \\
\hline $\mathrm{Te}_{2}$ & & 2.59 & 53 \\
\hline $\mathrm{Zn}_{2}$ & ${ }^{1} \sum ?$ & & 6 \\
\hline
\end{tabular}

a Data from G. Herzberg, Spectra of Diatomic Molecules, Van Nostrand, New York, 1950, Table 39; T. L. Cottrell, The Strengths of Chemical Bonds, Butterworths, London, 1958, Table 11.5.1; L. E. Sutton (ed.), "Interatomic Distances," Special Publication No. 11, The Chemical Society, London, 1958.

${ }^{b_{A}}$ short discussion of the ground state of $\mathrm{C}_{2}$ can be found in $\mathrm{J} . \mathrm{W}$. Linnett, Wave Mechanics and Valency, Methuen, London, 1960, p. 134.

The $\sigma^{3}$ orbital is shown in Fig. 2-18. The analytical expression for the $\sigma^{b} \mathrm{MO}$ of LiH has the form

$$
\psi\left(\sigma^{b}\right)=C_{1} 2 s+C_{2} 2 p_{z}+C_{3} 1 s
$$

In this case, $C_{3}>C_{1}>C_{2}$ and their numerical values are restricted by the normalization condition [Eq. (2-3)]. 


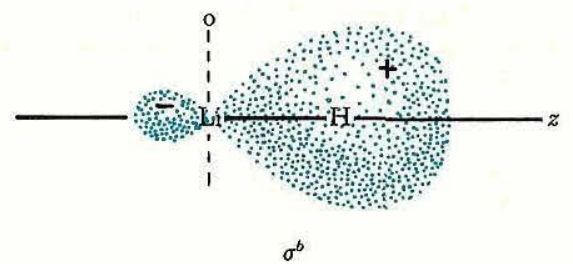

Figure 2-18 Boundary surface of the $\sigma$ bonding molecular orbital of $\mathrm{LiH}$.

Since both the $2 s$ and the $2 p_{z}$ lithium orbitals are used in the $\sigma$ molecular orbitals, there are two $\sigma^{*}$ orbitals, one involving the $2 s$ and one involving the $2 p_{z}$. These $\sigma^{*}$ orbitals are mainly localized on the $\mathrm{Li}$, as shown in Fig. 2-19. The approximate wave functions are:

$$
\begin{aligned}
& \psi\left(\sigma_{s}^{*}\right)=C_{4} 2 s-C_{5} 1 s ; \quad C_{4}>C_{5} \\
& \psi\left(\sigma_{z}^{*}\right)=C_{6} 2 p_{z}-C_{7} 1 s ; \quad C_{6}>C_{7}
\end{aligned}
$$
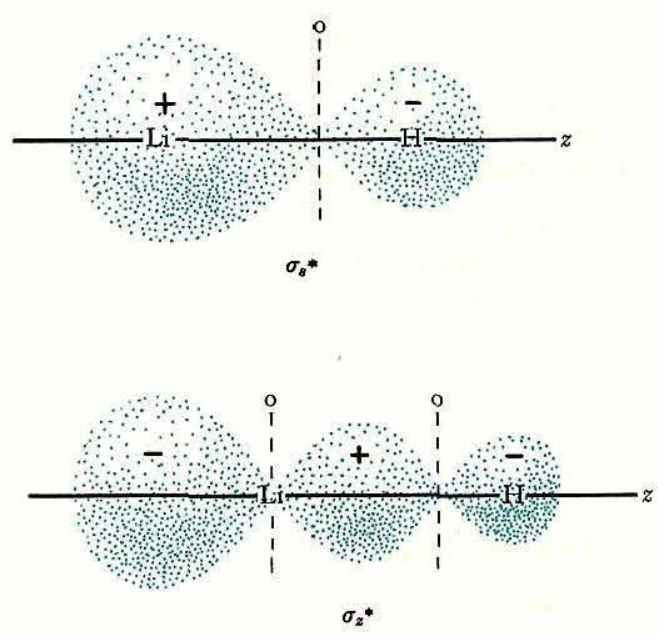

Figure 2-19 Boundary surfaces of the $\sigma_{s}{ }^{*}$ and $\sigma_{z}{ }^{*}$ MO's of LiH. 


\section{2-13 MOLECULAR-ORBITAL ENERGY-LEVEL SCHEME FOR LiH}

Figure 2-20 shows the MO energy-level scheme for $\mathrm{LiH}$. The valence orbitals of $\mathrm{Li}$ are placed on the left side of the diagram, with the $2 p$ level above the $2 s$ level. On the right side, the hydrogen $1 s$ level is shown. The $1 s$ level of $\mathrm{H}$ is placed below the $2 s$ level of $\mathrm{Li}$, to agree with their known stability difference.

The $\sigma^{b}$ and $\sigma^{*}$ MO's are placed in the center. The $\sigma^{b} \mathrm{MO}$ is more stable than the hydrogen $1 s$ valence orbital, and the diagram clearly shows that $\sigma^{b}$ is mainly composed of hydrogen $1 s$, with smaller fractions of lithium $2 s$ and $2 p_{z}$. The $\sigma_{s}{ }^{*} \mathrm{MO}$ is less stable than the lithium $2 s$ valence orbital, and the diagram shows that $\sigma_{s}{ }^{*}$ is composed of lithium $2 s$ and hydrogen $1 s$, with a much greater fraction of lith-

$\mathrm{Li}$ orbitals

LiH orbitals

$\mathrm{H}$ orbital

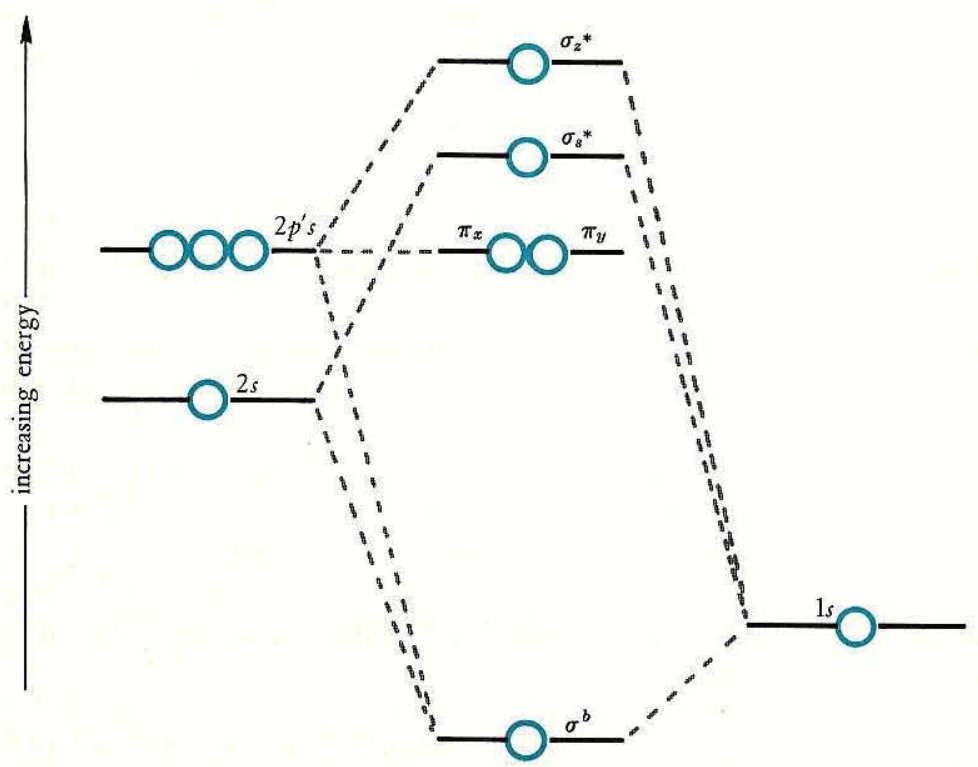

Figure 2-20 Relative orbital energies in $\mathrm{LiH}$. 
ium $2 s$. The $\sigma_{z}{ }^{*}$ orbital is shown less stable than $2 p_{z}$, and it clearly has considerable $2 p_{z}$ character.

The $2 p_{x}$ and $2 p_{y}$ orbitals of $\mathrm{Li}$ are shown in the $\mathrm{MO}$ column as $\pi$-type MO's. They are virtually unchanged in energy from the $\mathrm{Li}$ valence-orbital column, since $H$ has no valence orbitals capable of $\pi$-type interaction.

\section{2-14 GROUND State of LiH}

There are two electrons to place in the MO energy-level scheme for LiH shown in Fig. 2-20. This total is arrived at by adding together the one valence electron contributed by hydrogen $(1 s)$ and the one valence electron contributed by lithium $(2 s)$. Both electrons are accommodated in the $\sigma^{b} \mathrm{MO}$, giving a ground-state configuration

$$
\left(\sigma^{b}\right)^{2}=1 \Sigma
$$

Since the electrons in the $\sigma^{b} \mathrm{MO}$ spend more time in the vicinity of the $\mathrm{H}$ nucleus than of the $\mathrm{Li}$ nucleus, it follows that a separation of charge is present in the ground state. That is, the Li has a partial positive charge and the $\mathrm{H}$ has a partial negative charge, as shown below:

$$
\mathrm{Li}^{\delta+} \mathrm{H}^{\delta-}
$$

A limiting situation would exist if both electrons spent all their time around the $\mathrm{H}$. The $\mathrm{LiH}$ molecule in that case would be made up of a $\mathrm{Li}^{+}$ion and $\mathrm{H}^{-}$ion; that is, $\delta=1$. A molecule that can be formulated successfully as composed of ions is described as an ionic molecule. This situation is encountered in a diatomic molecule only if the valence orbital of one atom is very much more stable than the valence orbital of the other atom. The LiH molecule is probably not such an extreme case, and thus we say that $\mathrm{LiH}$ has partial ionic character. A calculation of the coefficients $C_{1}, C_{2}$, and $C_{3}$ would be required to determine the extent of this partial ionic character. One such calculation (unfortunately beyond the level of our discussion here) gives a charge distribution

$$
\mathrm{Li}^{0.8+} \mathrm{H}^{0.8-}
$$

which means that $\mathrm{LiH}$ has 80 per cent ionic character. 


\section{2-15 DIPOLE MOMENTS}

A heteronuclear diatomic molecule such as $\mathrm{LiH}$ possesses an electric dipole moment caused by charge separation in the ground state. This electric moment is equal to the product of the charge and the distance of separation,

$$
\text { dipole moment }=\mu=e R
$$

Taking $R$ in centimeters and $e$ in electrostatic units, $\mu$ is obtained in electrostatic units (esu). Since the unit of electronic charge is $4.8 \times$ $10^{-10} \mathrm{esu}$ and bond distances are of the order of $10^{-8} \mathrm{~cm}(1 \mathrm{~A})$, we see that dipole moments are of the order of $10^{-18} \mathrm{esu}$. It is convenient to express $\mu$ in Debye units (D), with $10^{-18} \mathrm{esu}=1$ Debye. If, as a first approximation, we consider the charges centered at each nucleus, $R$ in Eq. (2-37) is simply the equilibrium internuclear separation $R$ in the molecule.

Since it is possible to measure dipole moments, we have an experimental method of estimating the partial ionic character of heteronuclear diatomic molecules. The dipole moment of $\mathrm{LiH}$ is 5.9 Debye units (5.9D). For $R=1.60 \mathrm{~A}$ (or $1.60 \times 10^{-8} \mathrm{~cm}$ ), we calculate for an ionic structure $\mathrm{Li}^{+} \mathrm{H}^{-}$a dipole moment of $7.7 \mathrm{D}$. Thus the partial charge from the dipole moment datum is estimated to be $5.9 / 7.7=$ 0.77 , representing a partial ionic character of 77 per cent. This agrees with the theoretical value of 80 per cent given in the last section.

Dipole moments for a number of diatomic molecules are given in Table 2-7.

\section{2-16 ELECTRONEGATIVITY}

A particular valence orbital on one atom in a molecule which is more stable than a particular valence orbital on the other atom in a molecule is said to be more electronegative. A useful treatment of electronegativity was introduced by the American chemist Linus Pauling in the early 1930s. Electronegativity may be broadly defined as the ability of an atom in a molecule to attract electrons to itself. It must be realized, however, that each different atomic orbital in a molecule has a different electronegativity, and therefore atomic electronega- 
Table $2-7$

Dipole Moments of Some Diatomic Molecules ${ }^{a}$

\begin{tabular}{cc}
\hline Molecule & Dipole moment, $D$ \\
\hline $\mathrm{LiH}$ & 5.88 \\
$\mathrm{HF}$ & 1.82 \\
$\mathrm{HCl}$ & 1.07 \\
$\mathrm{HBr}$ & 0.79 \\
$\mathrm{HI}$ & 0.38 \\
$\mathrm{O}_{2}$ & 0 \\
$\mathrm{CO}$ & 0.12 \\
$\mathrm{NO}$ & 0.15 \\
$\mathrm{ICl}$ & 0.65 \\
$\mathrm{BrCl}$ & 0.57 \\
$\mathrm{FCl}$ & 0.88 \\
$\mathrm{FBr}$ & 1.29 \\
$\mathrm{KF}$ & 8.60 \\
$\mathrm{KI}$ & 9.24 \\
\hline
\end{tabular}

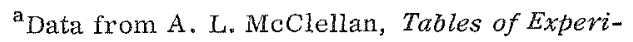
mental Dipole Moments, Freeman, San Francisco, 1963.

tivities vary from situation to situation, depending on the valence orbitals under consideration. Furthermore, the electronegativity of an atom in a molecule increases with increasing positive charge on the atom.

The Pauling electronegativity value for any given atom is obtained by comparing the bond-dissociation energies of certain molecules containing that atom, in the following way. The bond-dissociation energy (DE) of $\mathrm{LiH}$ is $58 \mathrm{kcal} /$ mole. The DE's of $\mathrm{Li}_{2}$ and $\mathrm{H}_{2}$ are 25 and $103 \mathrm{kcal} / \mathrm{mole}$, respectively. We know that the DE's of $\mathrm{Li}_{2}$ and $\mathrm{H}_{2}$ refer to the breaking of purely covalent bonds-that is, that the two electrons in the $\sigma^{b}$ levels are equally shared between the two hydrogen and the two lithium atoms, respectively. If the two electrons in the $\sigma^{b} \mathrm{MO}$ of $\mathrm{LiH}$ were equally shared between $\mathrm{Li}$ and $\mathrm{H}$, we might expect to be able to calculate the $\mathrm{DE}$ of $\mathrm{LiH}$ from the geometric mean; thus 


$$
\mathrm{DE}_{\mathrm{L} i \mathrm{H}} \stackrel{?}{=} \sqrt{\mathrm{DE}_{\mathrm{H}_{2}} \times \mathrm{DE}_{\mathrm{Li}_{2}}}
$$

This geometric mean is only $51 \mathrm{kcal} /$ mole, $7 \mathrm{kcal} /$ mole less than the observed $\mathrm{DE}$ of $\mathrm{LiH}$. It is a very general result that the $D E$ of a molecule $A B$ is almost always greater than the geometric mean of the $D E$ 's of $A_{2}$ and $B_{2}$. An example more striking than $\mathrm{LiH}$ is the system BF. The $D E$ 's of $B_{2}, F_{2}$, and $B F$ are 69,36 , and $195 \mathrm{kcal} /$ mole, respectively. The geometric mean gives

$$
\mathrm{DE}_{\mathrm{BF}} \stackrel{?}{=} \sqrt{69 \times 36}=50 \neq 195
$$

This "extra" bond energy in an AB molecule is presumably due to the electrostatic attraction of $A$ and $B$ in partial ionic form,

$$
\mathrm{A}^{\delta+} \mathrm{B}^{\delta-}
$$

Pauling calls the extra $\mathrm{DE}$ possessed by a molecule with partial ionic character the ionic resonance energ $y$ or $\Delta$. Thus we have the equation

$$
\Delta=\mathrm{DE}_{\mathrm{AB}}-\sqrt{\mathrm{D}_{A_{2}} \times \mathrm{D}_{B_{2}}}
$$

The electronegativity difference between the two atoms $A$ and $B$ is then defined as

$$
\chi_{A}-\chi_{B}=0.208 \sqrt{\Delta}
$$

where $\chi_{A}$ and $\chi_{B}$ are electronegativities of atoms $A$ and $B$ and the factor 0.208 converts from $\mathrm{kcal} /$ mole to electron-volt units. The square root of $\Delta$ is used because it gives a more nearly consistent set of electronegativity values for the atoms. Since only differences are obtained from the application of Eq. (2-41), one atomic electronegativity value must be arbitrarily agreed upon, and then all the others are easily obtained. On the Pauling scale, the most electronegative atom, fluorine, is assigned an electronegativity (or EN) of approximately 4. The most recent EN values, calculated using the Pauling idea, are given in Table 2-8.

Another method of obtaining EN values was suggested by R. S. Mulliken, an American physicist. Mulliken's suggestion is that atomic electronegativity is the arithmetic mean of the ionization potential and the electron affinity of an atom; i.e.,

$$
\mathrm{EN}=\frac{\mathrm{IP}+\mathrm{EA}}{2}
$$


Table 2-8

Atomic Electronegativities

\begin{tabular}{|c|c|c|c|c|c|c|c|c|c|c|c|c|c|c|c|c|}
\hline I & II & III & II & II & II & II & II & II & II & I & II & III & IV & III & II & I \\
\hline $\begin{array}{l}\mathrm{H} \\
2.20\end{array}$ & & & & & & & & & & & & & & & & \\
\hline $\begin{array}{l}\mathrm{Li} \\
0.98\end{array}$ & $\begin{array}{l}\mathrm{Be} \\
1.57\end{array}$ & & & & & & & & & & & $\begin{array}{l}\text { B } \\
2.04\end{array}$ & $\begin{array}{l}\mathrm{C} \\
2.55\end{array}$ & $\begin{array}{l}\mathrm{N} \\
3.04\end{array}$ & $\begin{array}{l}\mathrm{O} \\
3.44\end{array}$ & $\begin{array}{l}\mathrm{F} \\
3.98\end{array}$ \\
\hline $\begin{array}{l}\mathrm{Na} \\
0.93\end{array}$ & $\begin{array}{l}\mathrm{Mg} \\
1.31\end{array}$ & & & & & & & & & & & $\begin{array}{l}\mathrm{A} 1 \\
1.61\end{array}$ & $\begin{array}{l}\mathrm{Si} \\
1.90\end{array}$ & $\begin{array}{l}\mathrm{P} \\
2.19\end{array}$ & $\begin{array}{l}S \\
2.58\end{array}$ & $\begin{array}{l}\mathrm{Cl} \\
3.16\end{array}$ \\
\hline $\begin{array}{l}\mathrm{K} \\
0.82\end{array}$ & $\begin{array}{l}\mathrm{Ca} \\
1.00\end{array}$ & $\begin{array}{l}\mathrm{Sc} \\
1.36\end{array}$ & $\begin{array}{l}\mathrm{Ti} \\
1,54\end{array}$ & $\begin{array}{l}\mathrm{V} \\
1.63\end{array}$ & $\begin{array}{l}\mathrm{Cr} \\
1.66\end{array}$ & $\begin{array}{l}\mathrm{Mn} \\
1.55\end{array}$ & $\begin{array}{l}\mathrm{Fe} \\
1.83\end{array}$ & $\begin{array}{l}\text { Co } \\
1.88\end{array}$ & $\begin{array}{l}\mathrm{Ni} \\
1.91\end{array}$ & $\begin{array}{l}\mathrm{Cu} \\
1.90\end{array}$ & $\begin{array}{l}\mathrm{Zn} \\
\mathbf{1 . 6 5}\end{array}$ & $\begin{array}{l}\mathrm{Ga} \\
1.81\end{array}$ & $\begin{array}{l}\mathrm{Ge} \\
2.01\end{array}$ & $\begin{array}{l}\text { As } \\
2.18\end{array}$ & $\begin{array}{l}\mathrm{Se} \\
2.55\end{array}$ & $\begin{array}{l}\mathrm{Br} \\
2.96\end{array}$ \\
\hline $\begin{array}{l}\mathrm{Rb} \\
0.82\end{array}$ & $\begin{array}{l}\mathrm{Sr} \\
0.95\end{array}$ & $\begin{array}{l}\mathrm{Y} \\
1.22\end{array}$ & $\begin{array}{l}\mathrm{Zr} \\
1.33\end{array}$ & & $\begin{array}{l}\text { Mo } \\
2.16\end{array}$ & & & $\begin{array}{l}\mathrm{Rh} \\
2.28\end{array}$ & $\begin{array}{l}\mathrm{Pd} \\
2.20\end{array}$ & $\begin{array}{l}\mathrm{Ag} \\
1.93\end{array}$ & $\begin{array}{l}\mathrm{Cd} \\
1.69\end{array}$ & $\begin{array}{l}\text { In } \\
1.78\end{array}$ & $\begin{array}{l}\mathrm{Sn} \\
1.96\end{array}$ & $\begin{array}{l}\mathrm{Sb} \\
2.05\end{array}$ & & $\begin{array}{l}\text { I } \\
2.66\end{array}$ \\
\hline $\begin{array}{l}\mathrm{Cs} \\
0.79\end{array}$ & $\begin{array}{l}\mathrm{Ba} \\
0.89\end{array}$ & $\begin{array}{l}\mathrm{La} \\
1.10\end{array}$ & & & $\begin{array}{l}\mathrm{W} \\
2.36\end{array}$ & & & $\begin{array}{l}\text { Ir } \\
2.20\end{array}$ & $\begin{array}{l}\mathrm{Pt} \\
2.28\end{array}$ & $\begin{array}{l}\mathrm{Au} \\
2.54\end{array}$ & $\begin{array}{l}\mathrm{Hg} \\
2.00\end{array}$ & $\begin{array}{l}\mathrm{Tl} \\
2.04\end{array}$ & $\begin{array}{l}\mathrm{Pb} \\
2.33\end{array}$ & $\begin{array}{l}\mathrm{Bi} \\
2.02\end{array}$ & & \\
\hline & & $\begin{array}{l}\mathrm{Ce} \\
1.12\end{array}$ & $\begin{array}{l}\mathrm{Pr} \\
1.13 \\
\text { (III) }\end{array}$ & $\begin{array}{l}\mathrm{Nd} \\
1.14 \\
\text { (III) }\end{array}$ & & $\begin{array}{l}\text { Sm } \\
1.17 \\
\text { (III) }\end{array}$ & & $\begin{array}{l}\mathrm{Gd} \\
1.20 \\
\text { (III) }\end{array}$ & & $\begin{array}{l}\text { Dy } \\
1.22 \\
\text { (III) }\end{array}$ & $\begin{array}{l}\text { Ho } \\
1.23 \\
\text { (III) }\end{array}$ & $\begin{array}{l}\operatorname{Er} \\
1.24\end{array}$ & $\begin{array}{l}\mathrm{Tm} \\
1.25 \\
\text { (III) }\end{array}$ & & $\begin{array}{l}\mathrm{Lu} \\
1.27 \\
\text { (III) }\end{array}$ & \\
\hline & & & & & $\begin{array}{l}\mathrm{U} \\
1.38 \\
\text { (III) }\end{array}$ & $\begin{array}{l}\mathrm{Np} \\
1.36 \\
\text { (III) }\end{array}$ & $\begin{array}{l}\mathrm{Pu} \\
1.28 \\
\text { (III) }\end{array}$ & & & & & & & & & \\
\hline
\end{tabular}

${ }^{a}$ From A. L. Allred, J. Inorg. Nucl. Chem., 17, 215 (1961); roman numerals give the oxidation state of the atom in the molecules which were used in the calculations. 
Equation (2-42) averages the ability of an atom to hold its own valence electron and its ability to acquire an extra electron, Of course the EN values obtained from Eq. (2-42) differ numerically from the Pauling values, but if the Mulliken values are adjusted so that fluorine has an EN of about 4, there is generally good agreement between the two schemes. ${ }^{1}$

\section{2-17 IONIC BONDING}

The extreme case of unequal sharing of a pair of electrons in an $\mathrm{MO}$ is reached when one of the atoms has a very high electronegativity and the other has a very small ionization potential (thus a small $\mathrm{EN}$ ). In this case the electron originally belonging to the atom with the small IP is effectively transferred to the atom with the high EN,

$$
\mathrm{M} \cdot+\mathrm{X} \cdot \rightarrow \mathrm{M}^{+}: \mathrm{X}^{-}
$$

The bonding in molecules in which there is an almost complete electron transfer is described as ionic. An example of such an ionic diatomic molecule is lithium fluoride, $\mathrm{LiF}$. To a good approximation, the bond in $\mathrm{LiF}$ is represented as $\mathrm{Li}^{+} \mathrm{F}^{-}$. The energy required to completely separate the ions in a diatomic ionic molecule (Fig. 2-21) is given by the following expression:

potential energy $=$ electrostatic energy + van der Waals energy

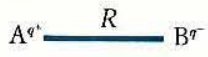

$$
\downarrow
$$

$\mathrm{A}^{7^{+}}$

$+$

$\mathrm{B}^{8^{-}}$

Figure 2-21 Dissociation of an ionic molecule into ions.

\footnotetext{
${ }^{1}$ However, note that the two scales are in different units.
} 
The electrostatic energy is

$$
\frac{q_{1} q_{2} e^{2}}{R}
$$

where $q_{1}$ and $q_{2}$ are charges on atoms $\mathrm{M}$ and $\mathrm{X}$ and $R$ is the internuclear separation.

There are two parts to the van der Waals energy. The most important at short range is the repulsion between electrons in the filled orbitals of the interacting atoms. This electron-pair repulsion is illustrated in Fig. 2-22. We have previously mentioned the mutual repulsion of filled inner orbitals, in comparing the bond energies of $\mathrm{Li}_{2}$ and $\mathrm{H}_{2}$.

The analytical expression commonly used to describe this interaction is

$$
\text { van der Waals repulsion }=b e^{-a R}
$$

where $b$ and $a$ are constants in a given situation. Notice that this repulsion term becomes very small at large $R$ values.

The other part of the van der Waals energy is the attraction that results when electrons in the occupied orbitals on the different atoms correlate their movements in order to avoid each other as much as possible. For example, as shown in Fig. 2-23, electrons in orbitals on atoms $\mathrm{M}$ and $\mathrm{X}$ can correlate their movements so that an instantaneous-dipole-induced-dipole attraction results. This type of potential

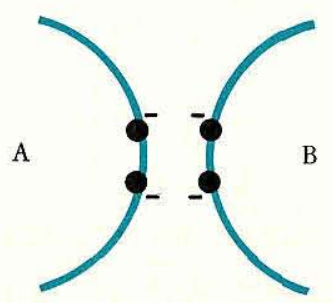

Figure 2-22 Repulsion of electrons in filled orbitals. This repulsion is very large when the filled orbitals overlap (recall the Pauli principle). 

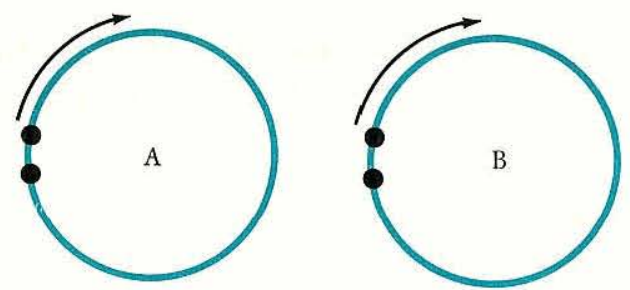

Figure 2-23 Schematic drawing of the instantaneousdipole-induced-dipole interaction, which gives rise to a weak attraction.

energy is known as the London energy, and is defined by the expression

$$
\text { London energy }=-\frac{d}{R^{6}}
$$

where $d$ is a constant for any particular case. The reciprocal $R^{6}$ type of energy term falls off rapidly with increasing $R$, but not nearly so rapidly as the $b e^{-a R}$ repulsion term. Thus the London energy is more important than the repulsion at longer distances.

\section{2-18 SIMPLE IONIC MODEL FOR THE ALKALI HALIDES}

The total potential energy for an ionic alkali halide molecule is given by the expression

$$
\mathrm{PE}=\frac{-q_{1} q_{2} e^{2}}{R}+. b e^{-a R}-\frac{d}{R^{6}}
$$

We need only know the values of the constants $b$, $a$, and $d$ in order to calculate potential energies from Eq. (2-47). The exact values of these constants for alkali metal ions and halide ions are not known. However, the alkali metal ions and the halide ions have inert-gas electronic configurations. For example, if $\mathrm{LiF}$ is formulated as an ionic molecule, $\mathrm{Li}^{+}$is isoelectronic with the inert gas $\mathrm{He}$, and $\mathrm{F}^{-}$is isoelectronic with the inert gas $\mathrm{Ne}$. Thus the van der Waals interaction in $\mathrm{Li}^{+} \mathrm{F}^{-}$may be considered approximately equal to the van 
der Waals interaction in the inert-gas pair He-Ne. This inert-gaspair approximation is of course applicable to the other alkali halide molecules as well.

The inert-gas-pair interactions can be measured and values for the $b, a$, and $d$ constants are available. These values are given in Table 2-9. Using Eq. (2-47), we are now able to calculate the bond energy of LiF.

EXAMPLE

To calculate the bond energy of LiF, we first calculate the energy needed for the process

$$
\mathrm{LiF} \rightarrow \mathrm{Li}^{+}+\mathrm{F}^{-}
$$

We shall calculate this energy in atomic units (au). The atomic unit of distance is the Bohr radius, $a_{0}$, or $0.529 \mathrm{~A}$. The atomic unit of charge is the electronic charge. The $b, a$, and $d$ constants in

Table 2-9

van der Waals Energy Parameters ${ }^{a}$

\begin{tabular}{cccc}
\hline Interaction pair & \multicolumn{1}{c}{$a$} & $b$ & $d$ \\
\hline $\mathrm{He}-\mathrm{He}$ & 2.10 & 6.55 & 2.39 \\
$\mathrm{He}-\mathrm{Ne}$ & 2.27 & 33 & 4.65 \\
$\mathrm{He}-\mathrm{Ar}$ & 2.01 & 47.9 & 15.5 \\
$\mathrm{He}-\mathrm{Kr}$ & 1.85 & 26.1 & 21.85 \\
$\mathrm{He}-\mathrm{Xe}$ & 1.83 & 42.4 & 33.95 \\
$\mathrm{Ne}-\mathrm{Ne}$ & 2.44 & 167.1 & 9.09 \\
$\mathrm{Ne}-\mathrm{Ar}$ & 2.18 & 242 & 30.6 \\
$\mathrm{Ne}-\mathrm{Kr}$ & 2.02 & 132 & 42.5 \\
$\mathrm{Ne}-\mathrm{Xe}$ & 2.00 & 214 & 66.1 \\
$\mathrm{Ar}-\mathrm{Ar}$ & 1.92 & 350 & 103.0 \\
$\mathrm{Ar}-\mathrm{Kr}$ & 1.76 & 191 & 143.7 \\
$\mathrm{Ar}-\mathrm{Xe}$ & 1.74 & 310 & 22.1 \\
$\mathrm{Kr}-\mathrm{Kr}$ & 1.61 & 104 & 200 \\
$\mathrm{Kr}-\mathrm{Xe}$ & 1.58 & 169 & 310 \\
$\mathrm{Xe}-\mathrm{Xe}$ & 1.55 & 274 & 480 \\
\hline
\end{tabular}

${ }^{\mathbf{a}}$ All values are in atomic units. Data from E. A. Mason, J. Chem. Phys., 23, 49 (1955). 
Table 2-9 are given in atomic units. Finally, $I$ au of energy is equal to $27.21 \mathrm{eV}$. The bond length of $\mathrm{LiF}$ is $1.52 \mathrm{~A}$; this is equal to 1.52 / $0.529=2.88 \mathrm{au}$. For $\mathrm{Li}^{+} \mathrm{F}^{-}, q_{1}=q_{2}=1$ au and $e^{2}=1 \mathrm{au}$.

Thus, on substitution of the $b, a$, and $d$ parameters for $\mathrm{He}-\mathrm{Ne}$, Eq. (2-47) becomes

$$
\mathrm{PE}=\frac{-1}{2.88}+33 e^{(-2.27)(2.88)}-\frac{4.65}{(2.88)^{6}}
$$

or

$$
\mathrm{PE}=-0.347+33(0.00144)-\frac{4.65}{571}
$$

or

$$
\mathrm{PE}=-0.308 \mathrm{au}=-8.38 \mathrm{eV}
$$

Accordingly, the energy required to separate $\mathrm{Li}^{+}$from $\mathrm{F}^{-}$at a bond distance of 2.88 au is $8.38 \mathrm{eV}$. This is called the coordinate-bond energy. However, we want to calculate the standard bond-dissociation energy, which refers to the process

$$
\mathrm{LiF} \stackrel{\mathrm{DE}}{\longrightarrow} \mathrm{Li}+\mathrm{F}
$$

That is, we need to take an electron from $\mathrm{F}^{-}$and transfer it to $\mathrm{Li}^{+}$:

$$
\mathrm{LiF} \stackrel{8.38 \mathrm{eV}}{\longrightarrow} \mathrm{Li}^{+}+\mathrm{F}^{-} \quad \stackrel{-\mathrm{IP}_{1}(\mathrm{Li})}{+\mathrm{EA}_{\mathrm{F}}} \mathrm{Li}+\mathrm{F}
$$

We see that the equation which allows us to calculate the DE of an alkali halide is

$$
\mathrm{DE}=-\mathrm{PE}-\mathrm{IP} \mathrm{P}_{1}+\mathrm{EA}
$$

Since $\mathrm{IP}_{1}(\mathrm{Li})=5.39 \mathrm{eV}$ and $E A_{F}=3.45 \mathrm{eV}$, we have finally

$$
\mathrm{DE}_{\mathrm{LiF}}=8.38-5.39+3.45=6.44 \mathrm{eV}
$$

The calculated $6.45 \mathrm{eV}$, or $149 \mathrm{kcal} /$ mole, compares favorably with the experimental DE of $137 \mathrm{kcal} /$ mole.

Experimental bond energies and bond distances for the alkali halide molecules are given in Table 2-10. The alkali halides provide the best examples of ionic bonding, since, of all the atoms, the alkali metals have the smallest IP's; of course the halogens help by having very high EN's. The most complete electron transfer would be expected 
Ta 10120

Bond Properties of the Alkall Halide ${ }^{a}$

\begin{tabular}{|c|c|c|}
\hline Molecule & Bond length, A & $\begin{array}{l}\text { Bond-dissociation } \\
\text { energy, kcal/mole }\end{array}$ \\
\hline $\mathrm{Cs}$ & 2.345 & 121 \\
\hline $\mathrm{CsCl}$ & 2.906 & 101 \\
\hline $\mathrm{CsBr}$ & 3.072 & 91 \\
\hline $\mathrm{CsI}$ & 3.315 & 75 \\
\hline$K F$ & $2.139^{b}$ & 118 \\
\hline $\mathrm{KCl}$ & 2.667 & 101 \\
\hline $\mathrm{KBr}$ & 2.821 & 91 \\
\hline $\mathrm{KI}$ & 3.048 & 77 \\
\hline $\operatorname{LiF}$ & $1.520^{b}$ & 137 \\
\hline $\mathrm{LiCl}$ & $2.029^{b}$ & 115 \\
\hline $\mathrm{LiBr}$ & 2.170 & 101 \\
\hline LiI & 2.392 & 81 \\
\hline $\mathrm{NaF}$ & $1.846^{b}$ & 107 \\
\hline $\mathrm{NaCl}$ & 2.361 & 98 \\
\hline $\mathrm{NaBr}$ & 2.502 & 88 \\
\hline $\mathrm{NaI}$ & 2.712 & 71 \\
\hline $\mathrm{RbF}$ & $2.242^{b}$ & 119 \\
\hline $\mathrm{RbCl}$ & 2.787 & 102 \\
\hline $\mathrm{RbBr}$ & 2.945 & 90 \\
\hline $\mathrm{RbI}$ & 3.177 & 77 \\
\hline
\end{tabular}

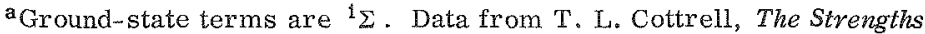
of Chemical Bonds, Butterworths, London, 1958, Table 11.5.1

bEstimated values; see L. Pauling, The Nature of the Chemical Bond, Cornell Univ. Press, Ithaca, N.Y., 1960, p. 532.

in CsF and the least complete in Lir. In LiI, covalent bonding may be of considerable importance.

\section{2-19 GeNerAL AB MOLECULES}

We shall now describe the bonding in a general diatomic molecule, $A B$, in which $B$ has a higher electronegativity than $A$, and both $A$ and $B$ have $s$ and $p$ valence orbitals. The molecular-orbital energy 
levels for $\mathrm{AB}$ are shown in Fig. 2-24. The $s$ and $p$ orbitals of $\mathrm{B}$ are placed lower than the $s$ and $p$ orbitals of $A$, in agreement with the electronegativity difference between $\mathrm{A}$ and $\mathrm{B}$. The $\sigma$ and $\pi$ bonding and antibonding orbitals are formed for $A B$ in the same manner as for $A_{2}$, but with the coefficients of the valence orbitals larger for $B$ in the bonding orbitals and larger for $\mathrm{A}$ in the antibonding orbitals. This means that the electrons in the bonding orbitals spend more time near the more electronegative $\mathrm{B}$. In the unstable antibonding orbitals, they spend more time near the less electronegative $A$. The
A orbitals
AB orbitals
B orbitals

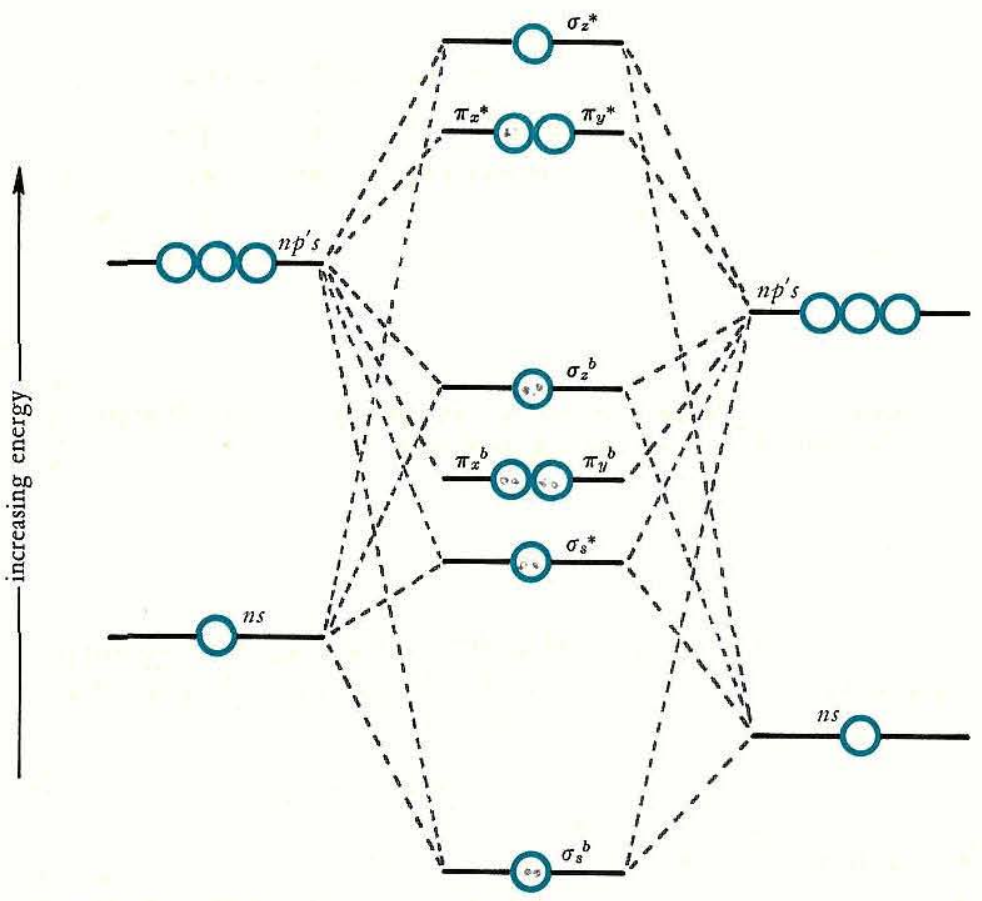

Figure 2-24 Relative orbital energies in a general AB molecule, with $B$ more electronegative than $A$. 

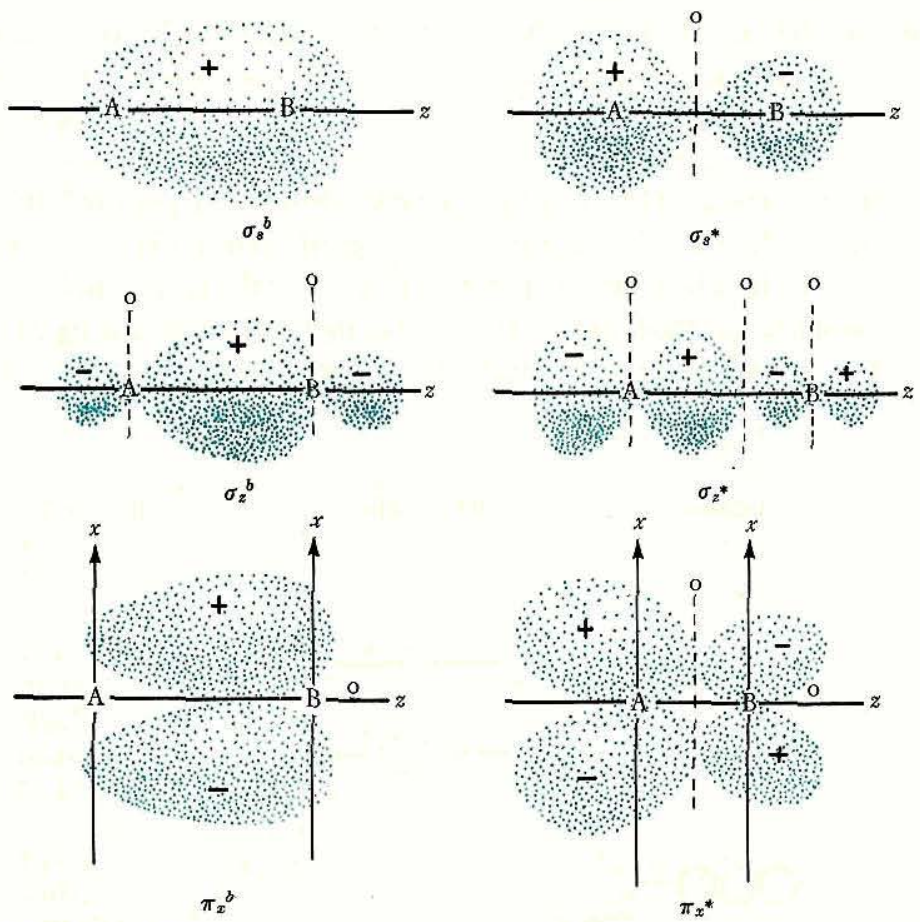

$\pi_{y}$ orbitals are equivalent to $\pi_{x}$

Figure 2-25 Boundary surfaces of the MO's of an AB molecule, with $B$ more electronegative than $A$.

boundary surfaces of the molecular orbitals for a general $A B$ molecule are given in Fig. 2-25. The following specific cases illustrate the use of the bonding scheme shown in Fig. 2-24.

\section{BN (8 Valence Electrons)}

The ground-state electronic configuration for $\mathrm{BN}$ is $\left(\sigma_{s}^{b}\right)^{2}\left(\sigma_{s}^{*}\right)^{2}$ $\left(\pi_{x, y^{b}}\right)^{3}\left(\sigma_{z}^{b}\right)$. This gives a ${ }^{3} \pi$ state and a prediction of two bonds 
$\left(\frac{1}{2} \sigma, \frac{3}{2} \pi\right)$. The BN molecule is thus electronically similar to $\mathrm{C}_{2}$. The bond lengths of $\mathrm{C}_{2}$ and $\mathrm{BN}$ are 1.31 and $1.28 \mathrm{~A}$, respectively. The $\mathrm{BN}$ bond energy is only $92 \mathrm{kcal} / \mathrm{mole}$, as compared to $150 \mathrm{kcal} /$ mole for $C_{2}$.

\section{$\mathrm{BO}, \mathrm{CN}, \mathrm{CO}^{+}(9$ Valence Electrons $)$}

The $\mathrm{BO}, \mathrm{CN}$, and $\mathrm{CO}^{+}$molecules all have the ground-state configuration $\left(\sigma_{s}^{b}\right)^{2}\left(\sigma_{s}^{*}\right)^{2}\left(\pi_{x, y^{b}}\right)^{4}\left(\sigma_{z}^{b}\right)$, and thus a ${ }^{2} \Sigma$ ground state. There are $2 \frac{1}{2}$ bonds predicted, which is $\frac{1}{2}$ more than for $\mathrm{BN}$. The bond lengths are all shorter than that of $\mathrm{BN}$ ( or $\mathrm{C}_{2}$ ), being $1.20 \mathrm{~A}$ for $\mathrm{BO}, 1.17 \mathrm{~A}$ for $\mathrm{CN}$, and $1.115 \mathrm{~A}$ for $\mathrm{CO}^{+}$. The bond energies are higher than that for $\mathrm{BN}$, being $185 \mathrm{kcal} / \mathrm{mole}$ for $\mathrm{BO}$ and $188 \mathrm{kcal} /$ mole for $\mathrm{CN}$.

\section{$\mathrm{CO}, \mathrm{NO}^{+}, \mathrm{CN}^{-}(10$ Valence Electrons $)$}

The $\mathrm{CO}, \mathrm{NO}^{+}$, and $\mathrm{CN}^{-}$molecules are isoelectronic with $\mathrm{N}_{2}$, having a ${ }^{1} \Sigma$ ground state. The configuration $\left(\sigma_{s}^{b}\right)^{2}\left(\sigma_{s}^{*}\right)^{2}\left(\pi_{x, y}\right)^{4}\left(\sigma_{z}^{b}\right)^{2}$ predicts one $\sigma$ and two $\pi$ bonds. The bond lengths of $\mathrm{NO}^{+}, \mathrm{CO}$, and $\mathrm{CN}^{-}$increase with increasing negative charge, being $1.062 \mathrm{~A}$ for $\mathrm{NO}^{+}, 1.128 \mathrm{~A}$ for $\mathrm{CO}$, and $1.14 \mathrm{~A}$ for $\mathrm{CN}^{-}$. Comparing molecules having the same charge, the bond lengths of $\mathrm{NO}^{+}, \mathrm{CO}$, and $\mathrm{CN}^{-}$are shorter than those of $\mathrm{BO}, \mathrm{CN}$, and $\mathrm{CO}^{+}$, as expected. The bond energy of $\mathrm{CO}$ is $255.8 \mathrm{kcal} /$ mole, which is even larger than the bond energy of $225 \mathrm{kcal} / \mathrm{mole}$ for $\mathrm{N}_{2}$.

\section{NO (11 Valence Electrons)}

The electronic configuration of NO is $\left(\sigma_{s}^{b}\right)^{2}\left(\sigma_{s}{ }^{*}\right)^{2}\left(\pi_{x, y^{b}}\right)^{4}\left(\sigma_{z}^{b}\right)^{2}$ $\left(\pi_{x, y^{*}}\right)$, giving a ${ }^{2} \pi$ ground state. Since the eleventh electron goes into a $\pi^{*}$ orbital, the number of bonds is now $2 \frac{1}{2}$, or $\frac{1}{2}$ less than for $\mathrm{NO}^{+}$. The bond length of NO is $1.15 \mathrm{~A}$, longer than either the $\mathrm{CO}$ or $\mathrm{NO}^{+}$distances. The bond energy of $\mathrm{NO}$ is $162 \mathrm{kcal} / \mathrm{mole}$, considerably less than the $\mathrm{CO}$ value.

The bond properties of a number of representative heteronuclear diatomic molecules are listed in Table 2-11. 


$$
\text { Table } 2=11
$$

Properties of Heteronuclear Diatomic Molecules ${ }^{a}$

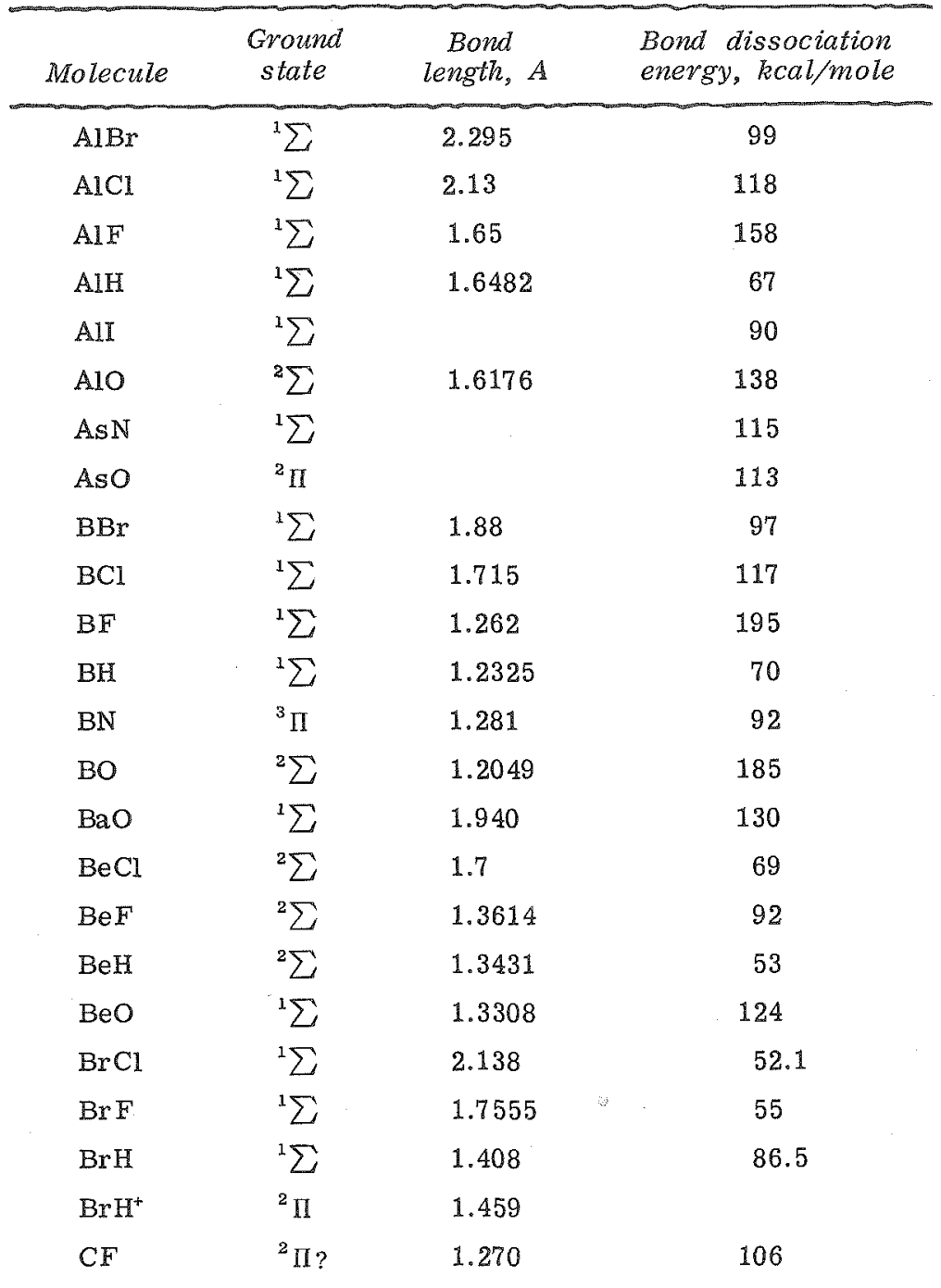


Table 2-11-(continued)

\begin{tabular}{|c|c|c|c|}
\hline Molecule & $\begin{array}{l}\text { Ground } \\
\text { state }\end{array}$ & $\begin{array}{l}\text { Bond } \\
\text { length, } A\end{array}$ & $\begin{array}{l}\text { Bond dissociation } \\
\text { energy, kcal/mole }\end{array}$ \\
\hline $\mathrm{CH}$ & ${ }^{2} \mathbb{I I}$ & 1.1198 & 80 \\
\hline $\mathrm{CN}$ & $2 \sum$ & 1.1718 & 188 \\
\hline $\mathrm{CN}^{+}$ & & 1.1727 & \\
\hline $\mathrm{CN}^{-}$ & & 1.14 & \\
\hline $\mathrm{CO}$ & ${ }^{1} \sum$ & 1.1282 & 255.8 \\
\hline $\mathrm{CO}^{+}$ & $2 \sum$ & 1.1151 & \\
\hline $\mathrm{CP}$ & ${ }^{2} \sum$ & 1.562 & 138 \\
\hline CS & ${ }^{1} \sum$ & 1.5349 & 166 \\
\hline CSe & ${ }^{1} \sum ?$ & 1.66 & 115 \\
\hline $\mathrm{CaO}$ & ${ }^{1} \sum$ & 1.822 & 100 \\
\hline $\mathrm{ClF}$ & ${ }^{1} \sum$ & 1.6281 & 60.5 \\
\hline $\mathrm{CsH}$ & ${ }^{1} \sum$ & 2.494 & 42 \\
\hline $\mathrm{GaCl}$ & ${ }^{1} \sum$ & 2.208 & 115 \\
\hline $\mathrm{GaF}$ & ${ }^{1} \sum$ & 1.775 & 142 \\
\hline GeO & ${ }^{1} \sum$ & 1.650 & 157 \\
\hline $\mathrm{HCl}$ & ${ }^{1} \sum$ & 1.2744 & 102.2 \\
\hline $\mathrm{HCl}^{+}$ & ${ }^{2} \Pi$ & 1.3153 & \\
\hline $\mathrm{HD}$ & ${ }^{1} \sum$ & 0.7413 & \\
\hline $\mathrm{HF}$ & ${ }^{1} \sum$ & 0.9175 & 134 \\
\hline $\mathrm{HI}$ & ${ }^{1} \sum$ & 1.608 & 70.5 \\
\hline HS & ${ }^{2} \Pi$ & 1.3503 & 80 \\
\hline $\mathrm{IBr}$ & ${ }^{1} \sum$ & & 41.90 \\
\hline $\mathrm{ICl}$ & ${ }^{1} \sum$ & 2.32070 & 49.63 \\
\hline $\mathrm{IF}$ & ${ }^{1} \sum$ & 1.985 & 46 \\
\hline $\operatorname{InBr}$ & ${ }^{1} \sum$ & 2.5408 & 85 \\
\hline
\end{tabular}


Table 2-11 (continued)

\begin{tabular}{|c|c|c|c|}
\hline Molecule & $\begin{array}{l}\text { Ground } \\
\text { state }\end{array}$ & $\begin{array}{l}\text { Bond } \\
\text { length, } A\end{array}$ & $\begin{array}{l}\text { Bond dissociation } \\
\text { energy, kcal/mole }\end{array}$ \\
\hline $\mathrm{InCl}$ & ${ }^{1} \Sigma$ & 2.4012 & 104 \\
\hline $\operatorname{In} F$ & ${ }^{1} \sum$ & 1.9847 & 125 \\
\hline InH & ${ }^{1} \Sigma$ & 1.8376 & 57 \\
\hline InI & ${ }^{1} \Sigma$ & 2.86 & 65 \\
\hline $\mathrm{KH}$ & ${ }^{1} \sum$ & 2.244 & 43 \\
\hline $\mathrm{LiH}$ & ${ }^{1} \sum$ & 1.5953 & 58 \\
\hline $\mathrm{MgO}$ & ${ }^{1} \sum$ & 1.749 & 92 \\
\hline $\mathrm{NH}$ & ${ }^{3} \sum$ & 1.038 & 85 \\
\hline $\mathrm{NH}^{+}$ & ${ }^{2} \Pi$ & 1.084 & \\
\hline NO & ${ }^{2} \Pi$ & 1.150 & 162 \\
\hline $\mathrm{NO}^{+}$ & ${ }^{1} \Sigma$ & 1.0619 & \\
\hline $\mathrm{NP}$ & ${ }^{1} \sum$ & 1.4910 & \\
\hline NS & ${ }^{2} \Pi$ & 1.495 & 115 \\
\hline $\mathrm{NS}^{+}$ & & 1.25 & \\
\hline $\mathrm{NaH}$ & ${ }^{1} \sum$ & 1.8873 & 47 \\
\hline $\mathrm{NaK}$ & ${ }^{1} \sum$ & & 14.3 \\
\hline $\mathrm{NaRb}$ & ${ }^{1} \sum$ & & 13.1 \\
\hline $\mathrm{OH}$ & ${ }^{2} \Pi$ & 0.9706 & 101.5 \\
\hline $\mathrm{OH}^{+}$ & ${ }^{3} \sum$ & 1.0289 & \\
\hline $\mathrm{PH}$ & ${ }^{3} \sum$ & 1.4328 & \\
\hline $\mathrm{PN}$ & ${ }^{1} \sum$ & 1.4910 & 138 \\
\hline PO & ${ }^{2} \Pi$ & 1.448 & 125 \\
\hline $\mathrm{PbH}$ & ${ }^{2} \Pi$ & 1.839 & 42 \\
\hline $\mathrm{PbO}$ & ${ }^{1} \sum$ & 1.922 & 94 \\
\hline $\mathrm{PbS}$ & ${ }^{1} \sum$ & 2.3948 & 75 \\
\hline
\end{tabular}


Table 2-11 (continued)

\begin{tabular}{|c|c|c|c|}
\hline Molecule & $\begin{array}{l}\text { Ground } \\
\text { state }\end{array}$ & $\begin{array}{c}\text { Bond } \\
\text { length, } A\end{array}$ & $\begin{array}{l}\text { Bond dissociation } \\
\text { energy, kcal/mole }\end{array}$ \\
\hline $\mathrm{RbH}$ & ${ }^{1} \sum$ & 2.367 & 39 \\
\hline SO & ${ }^{3} \sum$ & 1.4933 & 119 \\
\hline $\mathrm{SbO}$ & ${ }^{2} \Pi$ & & 74 \\
\hline $\mathrm{SiF}$ & ${ }^{2} \Pi$ & 1.603 & 88 \\
\hline $\mathrm{SiH}$ & ${ }^{2} \Pi$ & 1.520 & 74 \\
\hline $\mathrm{SiN}$ & ${ }^{2} \sum$ & 1.572 & 104 \\
\hline $\mathrm{SiO}$ & ${ }^{1} \sum$ & 1.509 & 185 \\
\hline SiS & ${ }^{1} \sum$ & 1.929 & 148 \\
\hline $\mathrm{SnH}$ & ${ }^{2} \Pi$ & 1.785 & 74 \\
\hline $\mathrm{SnO}$ & ${ }^{1} \sum$ & 1.838 & 132 \\
\hline SnS & ${ }^{1} \sum$ & 2.06 & 110 \\
\hline $\mathrm{SrO}$ & ${ }^{1} \sum$ & 1.920 & 83 \\
\hline $\mathrm{TIBr}$ & ${ }^{1} \sum$ & 2.6181 & 78 \\
\hline $\mathrm{TlCl}$ & ${ }^{1} \sum$ & 2.4848 & 87 \\
\hline $\mathrm{TIF}$ & ${ }^{1} \Sigma$ & 2.0844 & 109 \\
\hline $\mathrm{TIH}$ & ${ }^{1} \sum$ & 1.870 & 46 \\
\hline TII & ${ }^{1} \sum$ & 2.8136 & 65 \\
\hline
\end{tabular}

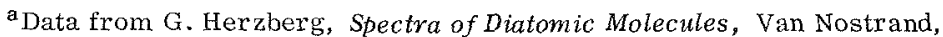
New York, 1950, Table 39; T. L. Cottrell, The Strengths of Chemical Bonds, Butterworths, London, 1958, Table 11.5.1; L. E. Sutton (ed.), "Interatomic Distances,' Special Publication No. 11, The Chemical Society, London, 1958.

\section{SUPPLEMENTARY PROBLEMS}

1. Find the ground-state term for (a) $\mathrm{B}_{2}$; (b) $\mathrm{F}_{2}$; (c) $\mathrm{C}_{2}$; (d) $\mathrm{S}_{2}$.

2. Discuss the bond properties of $\mathrm{N}_{2}, \mathrm{P}_{2}, \mathrm{As}_{2}, \mathrm{Sb}_{2}$, and $\mathrm{Bi}_{2}$ in terms of their electronic structures.

3. Discuss the bond properties of $\mathrm{Cl}_{2}$ and $\mathrm{Cl}_{2}+$ using molecularorbital theory. 
4. Calculate the bond energies of (a) CsF; (b) CsBr; (c) NaI; (d) $\mathrm{KCl}$. Compare your results with the experimental bond energies given in Table 2-10.

5. Work out the ground-state term for (a) BeF; (b) BeO. Calculate the bond energy of $\mathrm{BeO}$, assuming ionic bonding.

6. Discuss the bond properties of the interhalogen diatomic molecules- $\mathrm{ClF}, \mathrm{BrCl}, \mathrm{ICl}, \mathrm{IBr}$, etc.

7. Discuss the bond properties of NO, PO, AsO, and SbO.

8. Formulate the bonding in the hydrogen halide molecules (HF, $\mathrm{HCl}, \mathrm{HBr}$, and $\mathrm{HI}$ ) in terms of $\mathrm{MO}$ theory. Discuss the bond properties of these molecules. 


\section{III}

\section{Linear Triatomic Molecules}

\section{3-1 $\mathrm{BeH}_{2}$}

Tet us investigate the molecular orbitals of $\mathrm{BeH}_{2}$, a very simple 1. linear triatomic molecule. As in a diatomic molecule, we tag the molecular axis the 2 axis (the $\mathrm{H}-\mathrm{Be}-\mathrm{H}$ line), as shown in Fig. $3-1$. Beryllium has $2 s$ and $2 p$ valence orbitals; hydrogen has a $1 s$ valence orbital. The molecular orbitals for $\mathrm{BeH}_{2}$ are formed by using

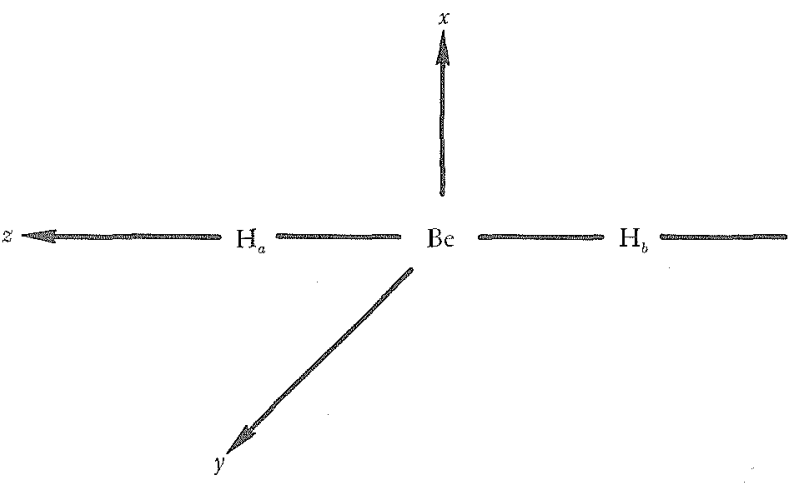

Figure 3-1 Coordinate system for $\mathrm{BeH}_{2}$. 


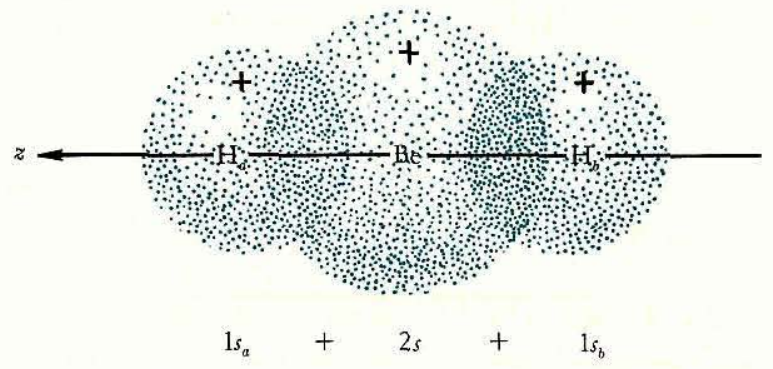

Figure 3-2 Overlap of the hydrogen $1 s$ orbitals with the beryllium $2 s$.

the $2 s$ and $2 p_{z}$ beryllium orbitals and the $1 s$ orbitals of $\mathrm{H}_{a}$ and $\mathrm{H}_{b}$. The proper linear combinations for the bonding molecular orbitals are obtained by writing the combinations of $1 s_{a}$ and $1 s_{b}$ that match the algebraic signs on the lobes of the central-atom (Be) $2 s$ and $2 p_{z}$ orbitals, respectively. This procedure gives a bonding orbital which concentrates electronic density between the nuclei. Since the $2 s$ orbital does not change sign over the boundary surface, the combination $\left(1 s_{a}+1 s_{b}\right)$ is appropriate (see Fig. $\left.3-2\right)$. The $2 p_{z}$ orbital has a plus lobe along $+z$ and $a$ minus lobe along $-z$. Thus the proper combination of $\mathrm{H}$ orbitals is $\left(1 s_{a}-1 s_{b}\right)$ (Fig. 3-3).

We have now described the two different $\sigma^{b}$ molecular orbitals,

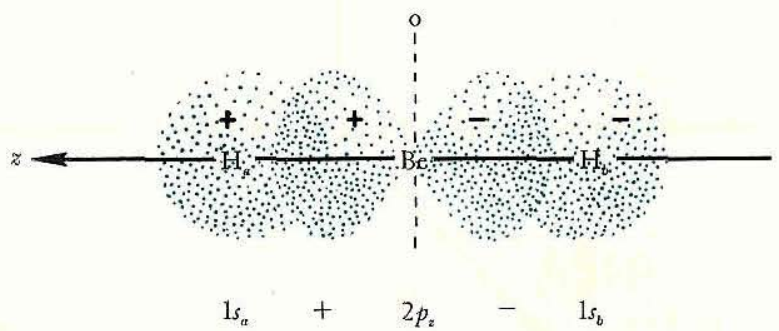

Figure 3-3 Overlap of the hydrogen $1 s$ orbitals with the beryllium $2 p_{z}$. 
which can be written as the following molecular-orbital wave functions:

$$
\begin{aligned}
& \psi\left(\sigma_{s}^{b}\right)=C_{1} 2 s+C_{2}\left(1 s_{a}+1 s_{b}\right) \\
& \psi\left(\sigma_{z}^{b}\right)=C_{3} 2 p_{z}+C_{4}\left(1 s_{a}-1 s_{b}\right)
\end{aligned}
$$

The antibonding molecular orbitals corresponding to $\psi\left(\sigma_{s}^{b}\right)$ and $\psi\left(\sigma_{z}^{b}\right)$ will have nodes between the Be and the two $H$ nuclei. That is, we shall combine the beryllium $2 s$ with $-\left(1 s_{a}+1 s_{b}\right)$ and the beryllium $2 p_{z}$ with $-\left(1 s_{a}-1 s_{b}\right)$. The two $\sigma^{*}$ molecular orbitals are therefore

$$
\psi\left(\sigma_{s}^{*}\right)=C_{5} 2 s-C_{6}\left(1 s_{a}+1 s_{b}\right)
$$

and

$$
\psi\left(\sigma_{z}^{*}\right)=C_{7} 2 p_{z}-C_{\delta}\left(1 s_{a}-1 s_{b}\right)
$$

In order to describe these $\sigma^{b}$ and $\sigma^{*}$ orbitals in more detail, we must find good numerical values for the coefficients of the Be and $\mathrm{H}$ valence orbitals. Though there are reasonably good approximate methods for doing this, all are beyond the level of this book. However, since the beryllium $2 s$ and $2 p_{z}$ orbitals are much less stable than the hydrogen $1 s$ orbitals ( $\mathrm{H}$ is more electronegative than $\mathrm{Be}$ ), we can confidently assume that the electrons in the bonding orbitals spend more time around the $\mathrm{H}$ nuclei-that is, that $2 C_{2}{ }^{2}>C_{1}{ }^{2}$ and $2 C_{4}{ }^{2}>C_{3}{ }^{2}$. In an antibonding orbital, an electron is forced to dwell mostly in the vicinity of the Be nuclets-that is, $C_{5}^{2}>2 C_{6}{ }^{2}$ and $C_{7}{ }^{2}>2 C_{8}{ }^{2}$. (For further explanation of the relationships between the coefficients, see Problem 3-1.)

The $2 p_{x}$ and $2 p_{y}$ beryllium orbitals are not used in bonding, since they are $\pi$ orbitals in a linear molecule and hydrogen has no $\pi$ valence orbitals. These orbitals are therefore nonbonding in the $\mathrm{BeH}_{2}$ molecule. The boundary surfaces of the $\mathrm{BeH}_{2}$ molecular orbitals are given in Fig. 3-4.

\section{3-2 ENERGY LEVELS FOR $\mathrm{BeH}_{2}$}

The molecular-orbital energy-level scheme for $\mathrm{BeH}_{2}$, shown in Fig. 3-5, is constructed as follows: The valence orbitals of the central atom are indicated on the left-hand side of the diagram, with 

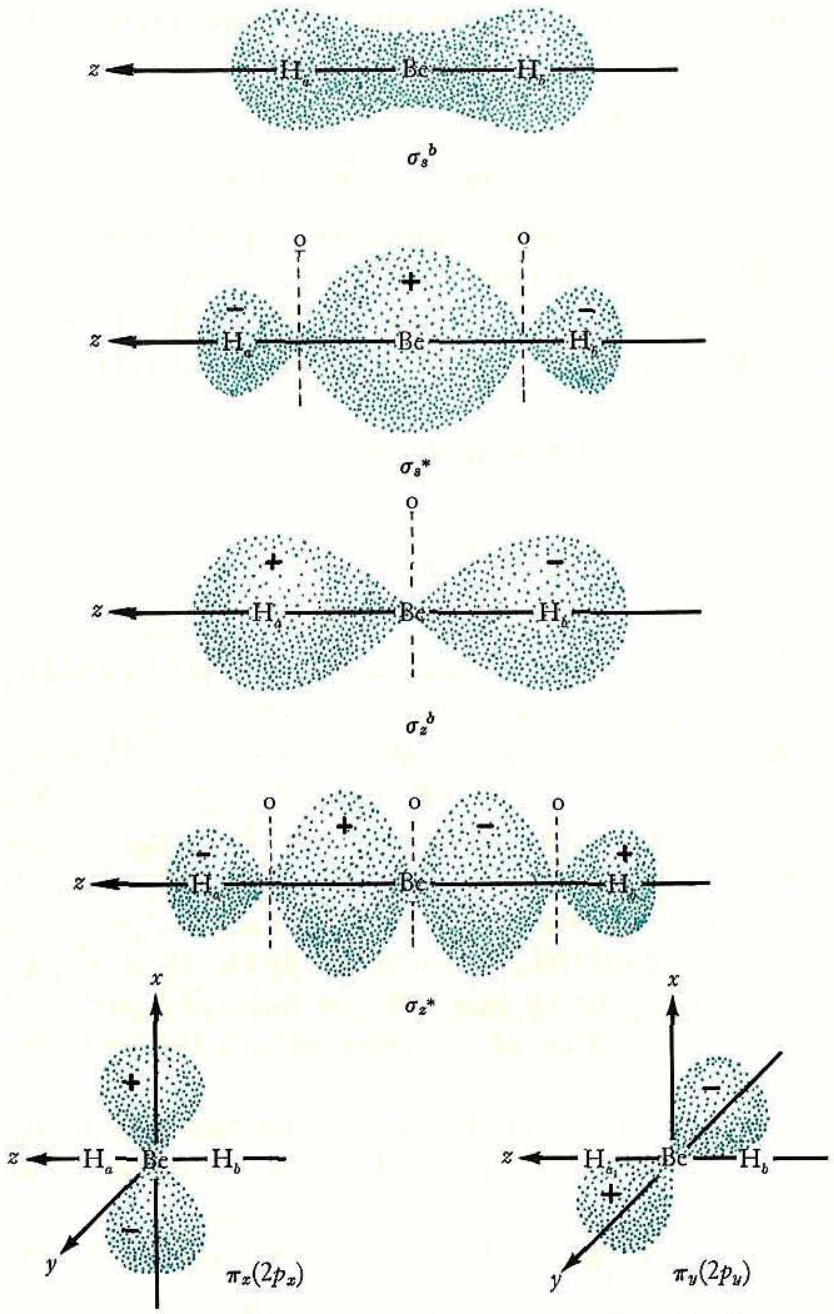

Figure 3-4 Boundary surfaces of the MO's of $\mathrm{BeH}_{2}$. 


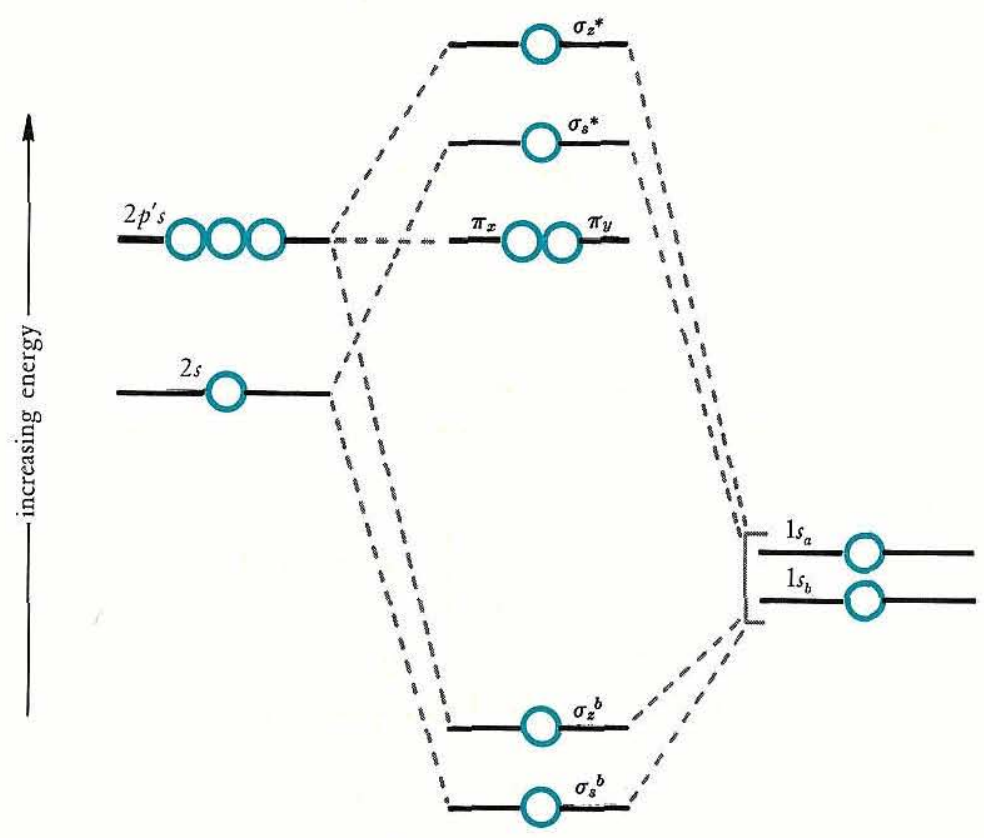

Figure 3-5 Relative orbital energies in $\mathbf{B e H}_{2}$.

the more stable $2 s$ level below the $2 p$. The $1 s$ orbitals of the two hydrogens are placed on the right-hand side of the diagram. The positioning of the $1 s$ hydrogen orbitals lower than either $2 s$ or $2 p$ of beryllium is based on EN considerations. In the middle of the diagram are the molecular orbitals - bonding, nonbonding, and antibonding. As usual, bonding levels are more stable than their combining atomic orbitals, and antibonding levels are correspondingly less stable. The $2 p_{x}$ and $2 p_{y}$ nonbonding Be orbitals are not changed in energy in our approximation scheme. Thus they are simply moved out into the molecular-orbital column.

The ground state of $\mathrm{BeH}_{2}$ is found by placing the valence electrons 
in the most stable molecular orbitals shown in Fig. 3-5. There are four valence electrons, two from beryllium $(2 s)^{2}$ and two from the two hydrogen atoms. The ground-state electronic configuration is therefore

$$
\left(\sigma_{s}^{b}\right)^{2}\left(\sigma_{z}^{b}\right)^{2}={ }^{1 \Sigma}
$$

\section{PROBLEM}

3-1. Assume that the electronic charge density is distributed in the $\sigma^{b}$ molecular orbitals as follows:

$$
\begin{aligned}
& \sigma_{s}^{b}: \text { Be, } 30 \text { per cent; } 2 \mathrm{H}, 70 \text { per cent } \\
& \sigma_{\varepsilon}{ }^{b} \text { : Be, } 20 \text { per cent; } 2 \mathrm{H}, 80 \text { per cent }
\end{aligned}
$$

Calculate the wave functions for $\sigma_{\varepsilon}^{b}$ and $\sigma_{z}^{b}$, as well as the final charge distribution in the $\mathrm{BeH}_{2}$ molecule.

Solution. Since the normalization condition is $\int|\psi|^{2} d \tau=1$, we have for $\sigma_{s}^{b}$

$$
\begin{aligned}
& \int\left|\psi\left(\sigma_{s}^{b}\right)\right|^{2} d \tau=C_{1}^{2} \mathcal{S}(2 s)^{2}{ }_{d r}+C_{2}^{2} \mathcal{S}\left(1 s_{a}\right)^{2} d \tau+C_{2}^{2} \mathcal{S}\left(1 s_{b}\right)^{2} d \tau \\
& +2 C_{1} C_{2} \int(2 s)\left(1 s_{a}\right) d \tau+2 C_{1} C_{2} \mathcal{S}(2 s)\left(1 s_{b}\right) d \tau \\
& +2 C_{2}^{2} \int\left(1 s_{a}\right)\left(1 s_{b}\right) d \tau=1
\end{aligned}
$$

If the atomic orbitals $2 s, 1 s_{a}$, and $1 s_{b}$ are separately normalized, we have

$$
\int\left|\psi\left(\sigma_{s}^{b}\right)\right|^{2} d \tau=C_{1}^{2}+C_{2}^{2}+C_{2}^{2}+\text { overlap terms }=1
$$

Making the simplifying assumption that the overlap terms are zero, we have finally

$$
\int\left|\psi\left(\sigma_{s}{ }^{b}\right\rangle\right|^{2} d \tau=C_{1}^{2}+2 C_{2}^{2}=1
$$

The probability for finding an electron in the $\sigma_{s}^{b}$ orbital if all space is examined is of course 1 . The equation $C_{1}^{2}+2 C_{2}^{2}=1$ shows that this total probability is divided, the term $C_{1}^{2}$ representing the probability for finding an electron in $\sigma_{s}{ }^{b}$ around $\mathrm{Be}$, and the term $2 C_{2}{ }^{2}$ the probability for finding an electron in $\sigma_{s}^{b}$ around the $\mathrm{H}$ atoms. Since the distribution of the electronic charge density is assumed to be 30 per cent for Be and 70 per cent for the $\mathrm{H}$ atoms in $\sigma_{s}^{b}$, the probabilities must be 0.30 for $\mathrm{Be}$ and 0.70 for the $\mathrm{H}$ atoms. Solving for the coefficients $C_{1}$ and $C_{2}$ in $\sigma_{s}^{b}$, we find

and

$$
C_{1}^{2}=0.30 \quad \text { or } \quad C_{1}=0.548
$$

$$
2 C_{2}^{2}=0.70 \quad \text { or } \quad C_{2}=0.592
$$


Similarly, we have the equation $C_{3}^{2}+2 C_{4}^{2}=1$ for $\sigma_{2}{ }^{h}$; again solving for coefficients on the basis of our electronic-charge-density assumptions,

$$
C_{3}^{2}=0.20 \quad \text { or } \quad C_{3}=0.447
$$

and

$$
2 C_{4}^{2}=0.80 \quad \text { or } \quad C_{4}=0.632
$$

The calculated wave functions are therefore

$$
\psi\left(\sigma_{s}^{b}\right)=(0.548) 2 s+0.592\left(1 s_{a}+1 s_{b}\right)
$$

and

$$
\psi\left(\sigma_{z}^{b}\right)=(0.447) 2 p_{z}+0.632\left(1 s_{a}-1 s_{b}\right)
$$

The ground-state configuration of $\mathrm{BeH}_{2}$ is $\left(\sigma_{s}^{b}\right)^{2}\left(\sigma_{z}^{b}\right)^{2}$. The distribution of these four valence electrons over the Be and $\mathrm{H}$ atoms is calculated as follows:

Be $\quad \sigma_{s}^{b}: \quad 2$ electrons $\times C_{1}{ }^{2}=2 \times 0.30=0.60$

$$
\begin{aligned}
\sigma_{2}{ }^{b} \text { : } \quad 2 \text { electrons } \times C_{3}{ }^{2}= & 2 \times 0.20=\frac{0.40}{\text { total }}
\end{aligned}
$$

$$
\begin{aligned}
\mathrm{H}_{a}=\mathrm{H}_{b} & \sigma_{s}^{b}: & 2 \text { electrons } \times C_{2}{ }^{2}= & 2 \times 0.35=0.70 \\
& \sigma_{z}^{b}: & 2 \text { electrons } \times C_{4}{ }^{2}= & 2 \times 0.40=0.80 \\
& & & \text { total }
\end{aligned}
$$

The $\mathrm{BeH}_{2}$ molecule without the four valence electrons is represented

$$
\mathrm{H}^{+}-\mathrm{Be}^{++}-\mathrm{H}^{+}
$$

Introducing the electrons as indicated above, we have the final charge distribution

$$
\stackrel{-0.5}{\mathrm{H}}-\stackrel{+}{-} \stackrel{\mathrm{Be}}{-}-\stackrel{-0.5}{-}
$$

It is most important to note from these calculations that the electronic charge densities associated witb the nuclei in a normalized molecular orbital are given by the squares of the coefficients of the atomic orbitals (in the zero-overlap approximation).

\section{3-3 VALENCE-BOND THEORY FOR $\mathrm{BeH}_{2}$}

The molecular-orbital description of $\mathrm{BeH}_{2}$ has the four electrons delocalized over all three atoms, in orbitals resembling the boundary- 
surface pictures shown in Fig. 3-4 $\left(\sigma_{s}^{b}\right.$ and $\left.\sigma_{z}^{b}\right)$. We may, however, cling to our belief in the localized two-electron bond and consider that the four valence electrons in $\mathrm{BeH}_{2}$ are in two equivalent bonding orbitals. By mixing together the $2 s$ and $2 p_{z}$ beryllium orbitals, we form two equivalent sp bybrid orbitals, as shown in Fig. 3-6. These two hybrid orbitals, $s p_{a}$ and $s p_{b}$, overlap nicely with $1 s_{a}$ and $1 s_{b}$, respectively, and the bonding orbitals are (see Fig. 3-7):

$$
\begin{aligned}
& \psi_{1}=C_{1} s p_{a}+C_{2} 1 s_{a} \\
& \psi_{2}=C_{1} s p_{b}+C_{2} 1 s_{b}
\end{aligned}
$$

The use of equivalent hybrid $\sigma$ orbitals for the central atom is especially helpful for picturing the $\sigma$ bonding in trigonal-planar and tetrahedral molecules.

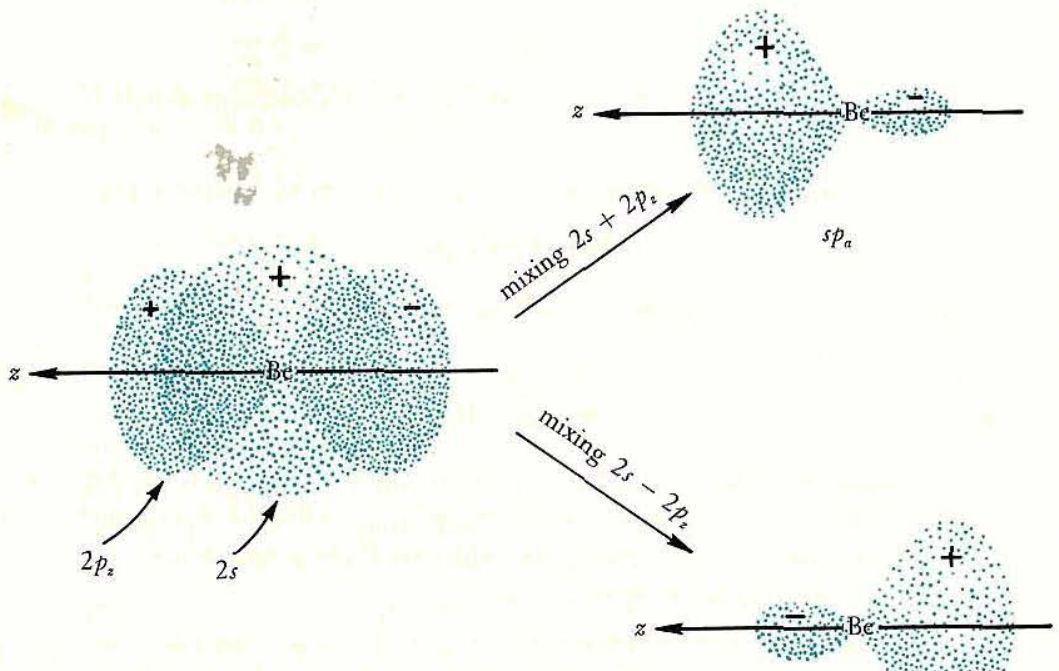

Figure 3-6 Formation of two sp hybrid orbitals. 


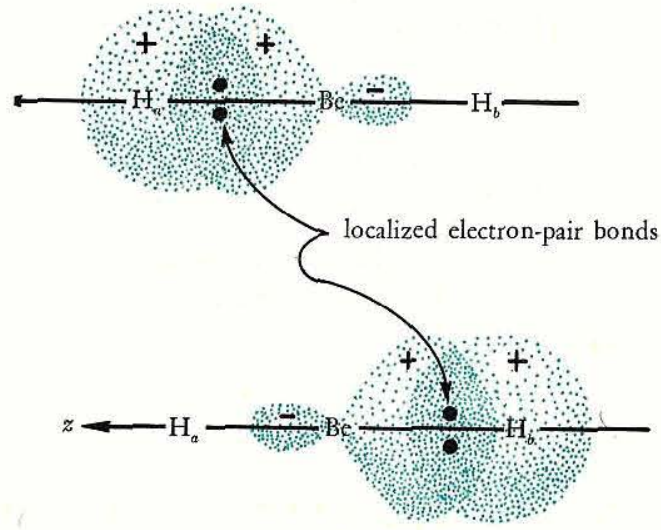

Figure 3-7 Valence bonds for $\mathrm{BeH}_{2}$, using two equivalent sp hybrid orbitals centered at the Be nucleus.

\section{PROBLEM}

3-2. Show that the general molecular-orbital description of $\mathrm{BeH}_{2}$ is equivalent to the valence-bond description if, in Eqs. (3-1) and (3-2), $C_{1}=C_{3}$ and $C_{2}=C_{4}$. (From the $\mathrm{MO}$ wave functions, construct the localized functions $\psi_{1}$ and $\psi_{2}$.)

\section{3-4 LINEAR TRIATOMIC MOLECULES WITH $\pi$ BONDING}

The $\mathrm{CO}_{2}$ molecule, in our standard coordinate system, is shown in Fig. 3-8. This molecule is an example of a linear triatomic molecule in which all three atoms have $n s$ and $n p$ valence orbitals. The $2 s$ and $2 p_{z}$ carbon orbitals are used for $\sigma$ bonding, along with the $2 p_{z}$ orbitals on each oxygen. ${ }^{1}$ The $\sigma$ orbitals are the same as for $\mathrm{BeH}_{2}$, except that now the end oxygen atoms use mainly the $2 p_{z}$ orbitals instead of the $1 s$ valence orbitals used by the hydrogen atoms. The $\sigma$ wave functions are:

$$
\psi\left(\sigma_{s}^{b}\right)=C_{1} 2 s+C_{2}\left(2 p_{z_{a}}+2 p_{z_{b}}\right)
$$

${ }^{1}$ The oxygen valence orbitals are $2 s$ and $2 p$. Thus a much better, approximate $\sigma$ MO scheme would include both $2 s$ and $2 p_{z}$ oxygen orbitals. For simplicity, however, we shall only use the $2 p_{z}$ oxygen orbitals in forming the $\sigma$ MO's. 


$$
\begin{aligned}
& \psi\left(\sigma_{s}^{*}\right)=C_{3} 2 s-C_{4}\left(2 p_{z_{a}}+2 p_{z_{b}}\right) \\
& \psi\left(\sigma_{z}^{b}\right)=C_{5} 2 p_{z}+C_{6}\left(2 p_{z_{a}}-2 p_{z_{b}}\right) \\
& \psi\left(\sigma_{z}^{*}\right)=C_{7} 2 p_{z}-C_{8}\left(2 p_{z_{a}}-2 p_{z_{b}}\right)
\end{aligned}
$$

The $\pi$ molecular orbitals are made up of the $2 p_{x}$ and $2 p_{y}$ valence orbitals of the three atoms. Let us derive the $\pi_{x}$ orbitals for $\mathrm{CO}_{2}$. There are two different linear combinations of the oxygen $2 p_{x}$ orbitals:

$$
\begin{aligned}
& 2 p_{x_{a}}+2 p_{x_{b}} \\
& 2 p_{x_{a}}-2 p_{x_{x_{b}}}
\end{aligned}
$$

The combination $\left(2 p_{x_{a}}+2 p_{x_{b}}\right)$ overlaps the carbon $2 p_{x}$ orbital as shown in Fig. 3-9. Since $x$ and $y$ are equivalent, we have the following $\pi^{b}$ and $\pi^{*}$ molecular orbitals:

$$
\begin{aligned}
& \psi\left(\pi_{x}{ }^{b}\right)=C_{92} 2 p_{y}+C_{10}\left(2 p_{x_{a}}+2 p_{x_{b}}\right) \\
& \psi\left(\pi_{y}^{b}\right)=C_{9} 2 p_{y}+C_{10}\left(2 p_{y_{a}}+2 p_{y_{b}}\right) \\
& \psi\left(\pi_{x}^{*}\right)=C_{11} 2 p_{x}-C_{12}\left(2 p_{x_{a}}+2 p_{x_{b}}\right) \\
& \psi\left(\pi_{y}^{*}\right)=C_{112} 2 p_{y}-C_{12}\left(2 p_{y_{a}}+2 p_{y_{b}}\right)
\end{aligned}
$$

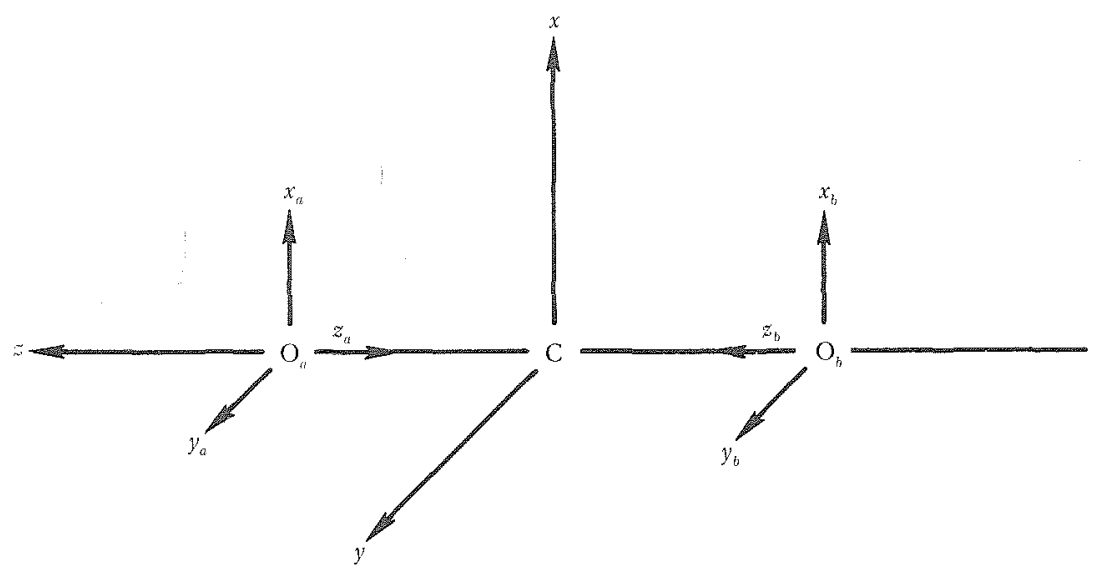

Figure $3=8$ Coordinate system for $\mathrm{CO}_{2}$. 

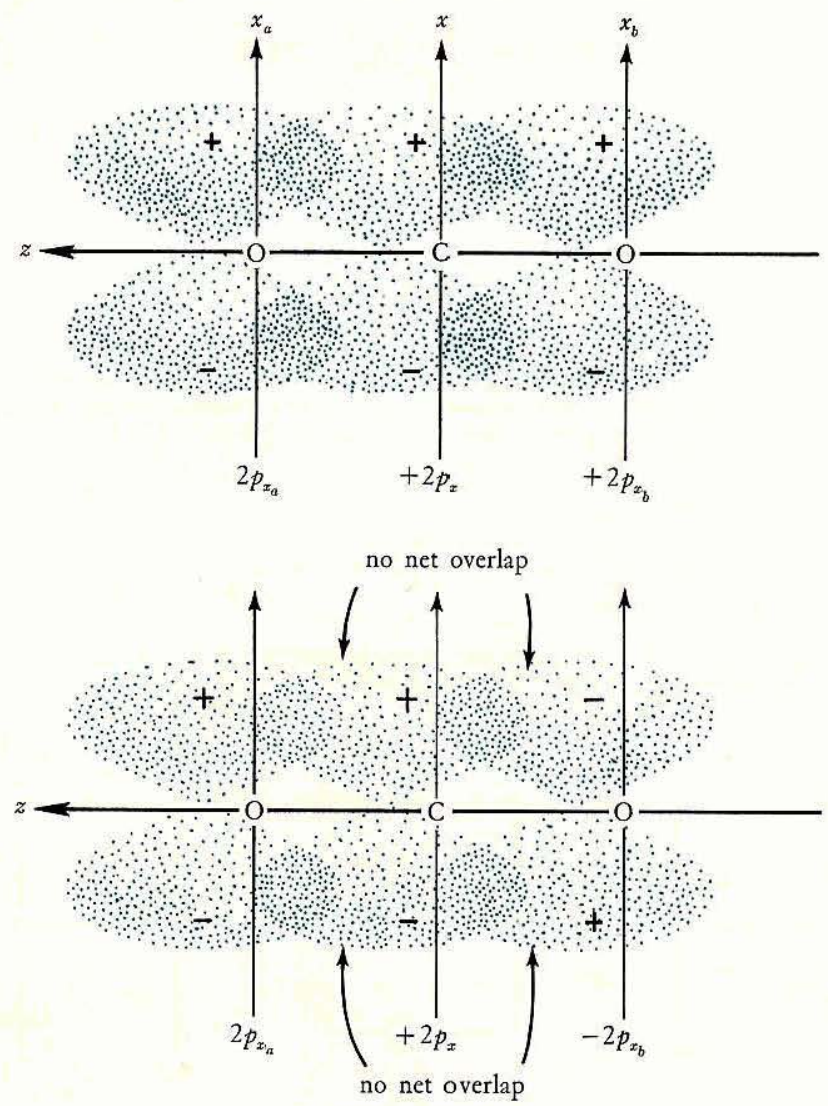

Figure 3-9 Overlap of the $2 p_{x}$ orbitals of the carbon atom and the two oxygen atoms.

The combination $\left(2 p_{x_{a}}-2 p_{x_{b}}\right)$ has zero overlap with the carbon $2 p_{x}$ orbital (see Fig. 3-9), and is therefore nonbonding in the molecularorbital scheme. We have, then, the normalized wave functions

$$
\psi\left(\pi_{x}\right)=\frac{1}{\sqrt{2}}\left(2 p_{x_{a}}-2 p_{x_{b}}\right)
$$

and 


$$
\psi\left(\pi_{y}\right)=\frac{1}{\sqrt{2}}\left(2 p_{y_{a}}-2 p_{y_{b}}\right)
$$

The boundary surfaces of the MO's for $\mathrm{CO}_{2}$ are shown in Fig. 3-10. The $\mathrm{MO}$ energy-level scheme for $\mathrm{CO}_{2}$ is given in Fig. 3-11. Notice
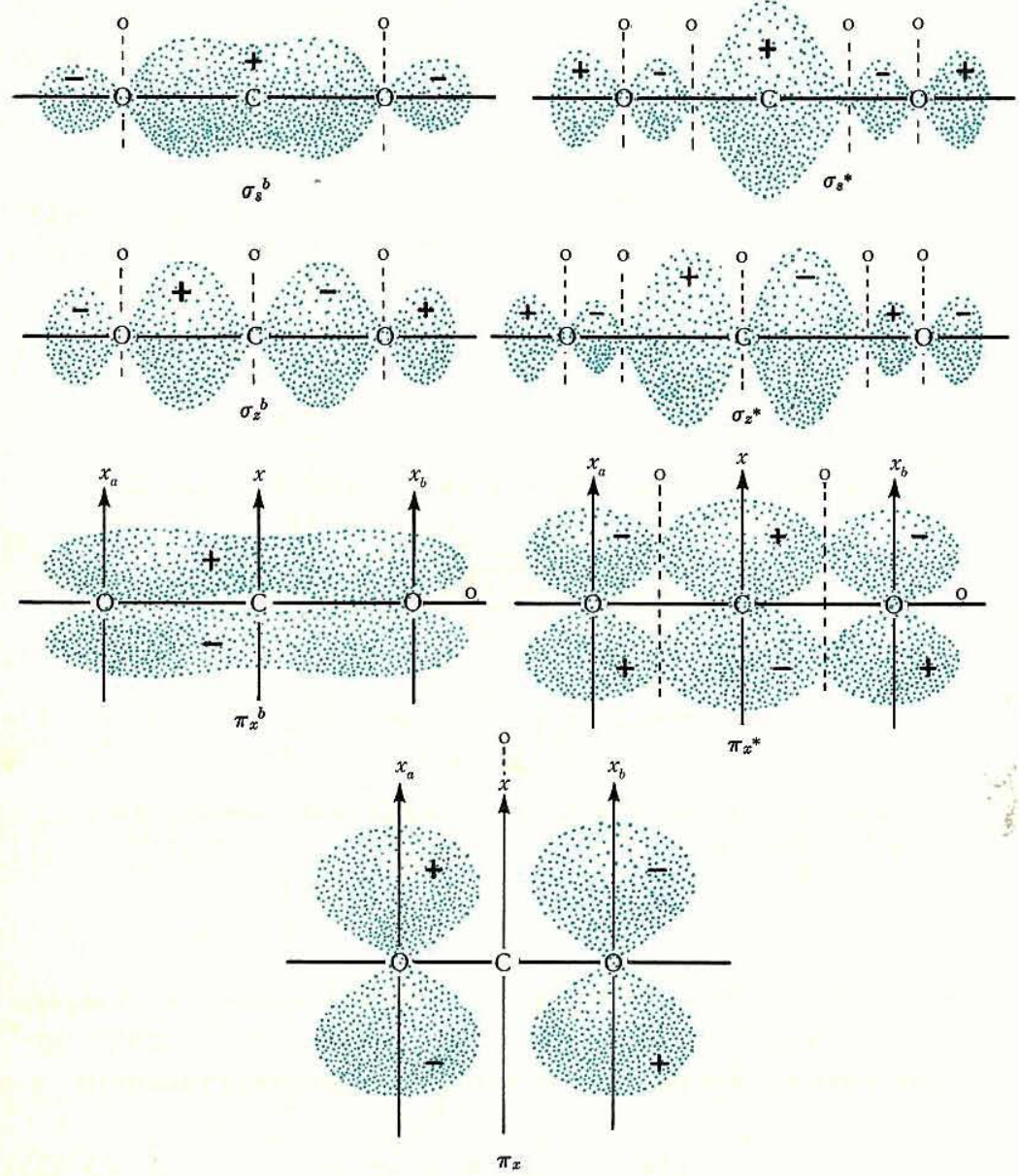

$\pi_{y}^{b}, \pi_{y^{*}}$, and $\pi_{y}$ are equivalent to $\pi_{x}^{b}, \pi_{x}^{*}$, and $\pi_{x}$

Figure 3-10 Boundary surfaces of the MO's of $\mathrm{CO}_{2}$. 


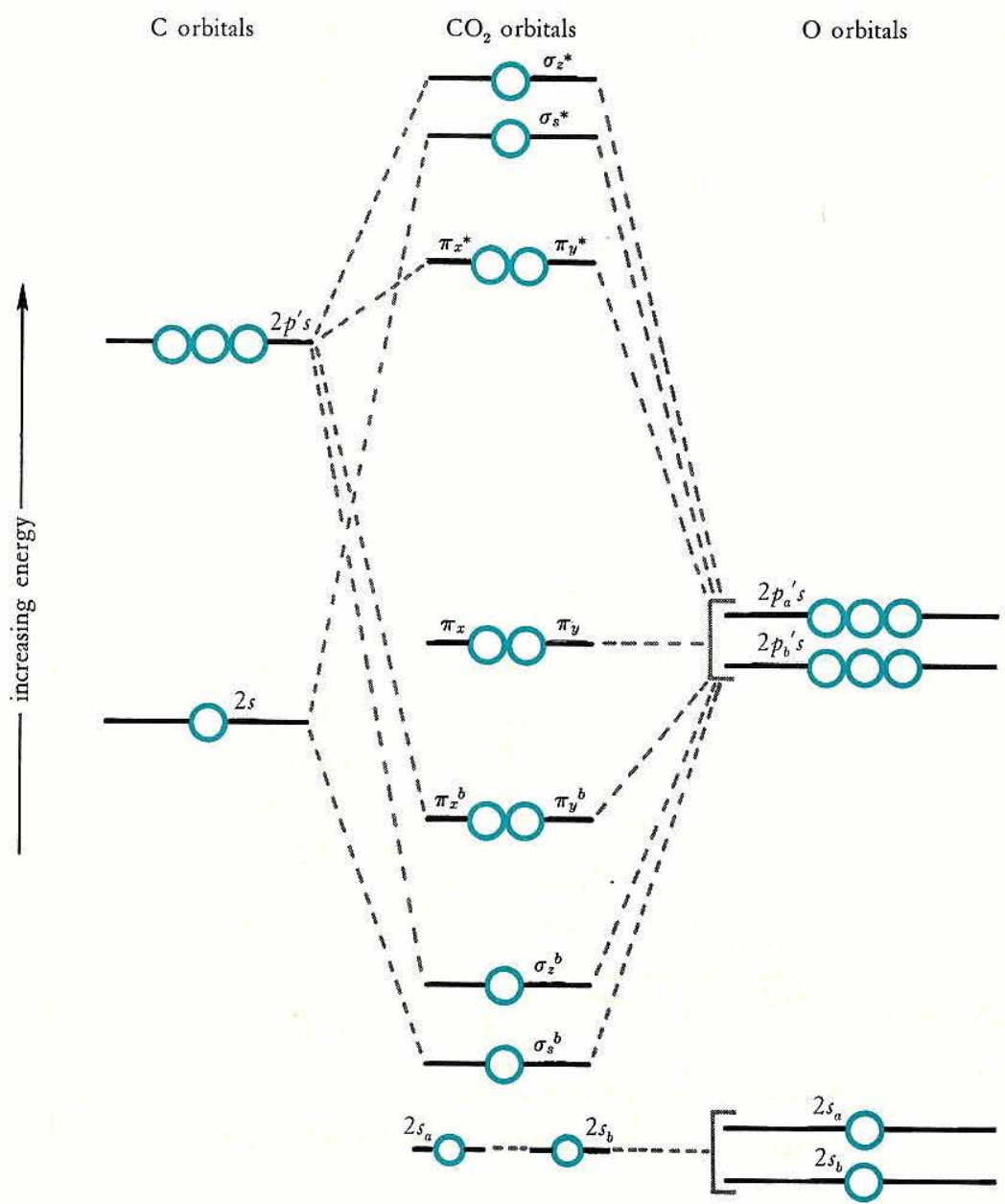

Figure 3-11 Relative orbital energies in $\mathrm{CO}_{2}$.

that the oxygen orbitals are more stable than the carbon orbitals. There are 16 valence electrons ( $\mathrm{C}$ is $2 s^{2} 2 p^{2}$; $\mathrm{O}$ is $2 s^{2} 2 p^{4}$ ) to place in the levels shown in the scheme. The ground, state of $\mathrm{CO}_{2}$ is therefore

$$
\left(2 s_{a}\right)^{2}\left(2 s_{b}\right)^{2}\left(\sigma_{s}^{b}\right)^{2}\left(\sigma_{z}^{b}\right)^{2}\left(\pi_{x, y}\right)^{4}\left(\pi_{x, y}\right)^{4} \quad{ }^{1} \Sigma
$$



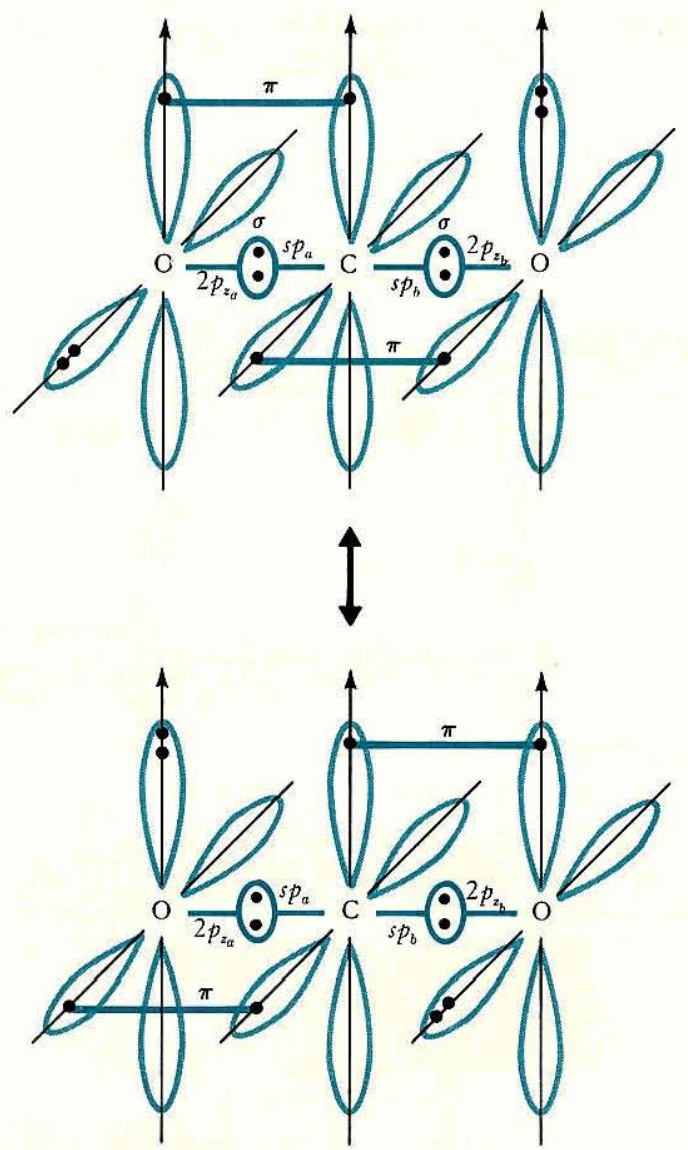

Figure 3-12 Valence-bond structures for $\mathrm{CO}_{2}$.

There are four electrons in $\sigma^{b}$ orbitals and four electrons in $\pi^{b}$ orbitals. Thus we have two $\sigma$ bonds and two $\pi$ bonds for $\mathrm{CO}_{2}$, in agreement with the two valence-bond structures shown in Fig. 3-12.

\section{3-5 BOND PROPERTIES OF $\mathrm{CO}_{2}$}

The $\mathrm{C}-\mathrm{O}$ bond distance in carbon dioxide is $1.162 \mathrm{~A}$, longer than the $\mathrm{C}-\mathrm{O}$ bond distance in carbon monoxide. These bond lengths 
are consistent with the double bond $(\mathrm{C}=\mathrm{O})$ between $\mathrm{C}$ and $\mathrm{O}$ in $\mathrm{CO}_{2}$ and the triple bond $(\mathrm{C} \equiv \mathrm{O})$ in $\mathrm{CO}$.

There are two types of bond energies for $\mathrm{CO}_{2}$. The bond-dissociation energy, which we discussed in Chapter II, refers to the breaking of a specific bond. In $\mathrm{CO}_{2}$, the process

$$
\mathrm{O}-\mathrm{C}-\mathrm{O} \stackrel{\mathrm{DE}}{\longrightarrow} \mathrm{CO}+\mathrm{O}
$$

represents the dissociation of one oxygen from carbon dioxide, leaving carbon monoxide; this DE is $127 \mathrm{kcal} /$ mole. However, the average $\mathrm{C}-\mathrm{O}$ bond energy in $\mathrm{CO}_{2}$ is obtained by completely splitting $\mathrm{CO}_{2}$ into ground-state atoms, breaking both $\mathrm{C}-\mathrm{O}$ bonds:

$$
\mathrm{O}-\mathrm{C}-\mathrm{O} \stackrel{E}{\longrightarrow} \mathrm{C}+\mathrm{O}+\mathrm{O}
$$

The average $\mathrm{C}-\mathrm{O}$ bond energy $(\mathrm{BE})$ is then one-half the value of $E$ in Eq. (3-20). Obviously $E$ is the sum of $\mathrm{DE}\left(\mathrm{CO}_{2}\right)$ and $\mathrm{DE}(\mathrm{CO})$,

$$
\begin{aligned}
& \mathrm{O}-\mathrm{C}-\mathrm{O} \stackrel{\mathrm{DE}\left(\mathrm{CO}_{2}\right)}{\longrightarrow} \mathrm{C}-\mathrm{O}+\mathrm{O} \stackrel{\mathrm{DE}(\mathrm{CO})}{\longrightarrow} \mathrm{C}+\mathrm{O}+\mathrm{O}(3-21) \\
& E=\mathrm{DE}\left(\mathrm{CO}_{2}\right)+\mathrm{DE}(\mathrm{CO})=127+256=383 \mathrm{kcal} / \mathrm{mole}
\end{aligned}
$$

and

$$
\frac{E}{2}=\mathrm{BE}\left(\mathrm{CO}_{2}\right) \cong 192 \mathrm{kcal} / \mathrm{mole}
$$

We shall use the abbreviations BE and DE in the bond-energy tables in this book.

The ground states, bond lengths, and bond energies for a number of linear triatomic molecules are given in Table 3-1.

36 IONIC TRIATOMIC MOLECULES: THE ALKALINE EARTH HALIDES

Molecules composed of atoms of the alkaline earth elements (Be, $\mathrm{Mg}, \mathrm{Ca}, \mathrm{Sr}, \mathrm{Ba}$ ) and halogen atoms are probably best described with the ionic model, since the electronegativity differences between alkaline earth and halogen a toms are large. Thus we picture the bonding as $\mathrm{X}^{-}-\mathrm{M}^{++}-\mathrm{X}^{-}$. Let us illustrate bond-energy calculations for molecules of this type, using $\mathrm{CaCl}_{2}$ as an example. 
Table $3-1$

Properties of Linear Triatomic Molecules ${ }^{a}$

\begin{tabular}{|c|c|c|c|c|}
\hline Molecule & $\begin{array}{l}\text { Ground } \\
\text { state }\end{array}$ & Bond & $\begin{array}{c}\text { Bond } \\
\text { length, A }\end{array}$ & $\begin{array}{c}\text { Bond energies, } \\
\text { kcal/mole }\end{array}$ \\
\hline $\mathrm{BeBr}_{2}$ & ${ }^{1} \Sigma$ & $\begin{array}{r}\mathrm{BrBe}-\mathrm{Br} \\
\mathrm{Be}-\mathrm{Br}\end{array}$ & & $89(\mathrm{BE})$ \\
\hline $\mathrm{BeCl}_{2}$ & ${ }^{1} \sum$ & $\begin{array}{r}\mathrm{ClBe}-\mathrm{Cl} \\
\mathrm{Be}-\mathrm{Cl}\end{array}$ & 1.74 & $\begin{array}{l}147(\mathrm{DE}) \\
109(\mathrm{BE})\end{array}$ \\
\hline $\mathrm{Bel}_{2}$ & ${ }^{1} \Sigma$ & $\begin{array}{r}\mathrm{IBe}-\mathrm{I} \\
\mathrm{Be}-\mathrm{I}\end{array}$ & & $69(\mathrm{BE})$ \\
\hline $\mathrm{CO}_{2}$ & ${ }^{1} \sum$ & $\begin{array}{r}O C-O \\
C-O\end{array}$ & 1.182 & $\begin{array}{l}127(\mathrm{DE}) \\
192(\mathrm{BE})\end{array}$ \\
\hline $\cos$ & ${ }^{1} \Sigma$ & OC-S & 1.561 & $128(\mathrm{DE})$ \\
\hline $\mathrm{CS}_{2}$ & ${ }^{1} \sum$ & $\begin{array}{r}S C-S \\
C-S\end{array}$ & 1.554 & $128(\mathrm{BE})$ \\
\hline $\mathrm{CSe}_{2}$ & ${ }^{1} \sum$ & $\mathrm{C}-\mathrm{Se}$ & & $112(B E)$ \\
\hline $\mathrm{CaCl}_{2}$ & $\sum$ & $\begin{array}{r}\mathrm{ClCa}-\mathrm{Cl} \\
\mathrm{Ca}-\mathrm{Cl}\end{array}$ & 2.54 & $\begin{array}{l}176(\mathrm{DE}) \\
113(\mathrm{BE})\end{array}$ \\
\hline $\mathrm{CdBr}_{2}$ & ${ }^{1} \sum$ & $\mathrm{Br} \mathrm{Cd}-\mathrm{Br}$ & 2.39 & $76(\mathrm{DE})$ \\
\hline $\mathrm{CdCl}_{2}$ & ${ }^{1} \sum$ & $\mathrm{ClCd}-\mathrm{Cl}$ & 2.23 & $84(\mathrm{DE})$ \\
\hline $\mathrm{CdI}_{2}$ & ${ }^{1} \sum$ & $I C d-I$ & 2.58 & $50(\mathrm{DE})$ \\
\hline $\mathrm{HCN}$ & ${ }^{1} \sum$ & $\begin{array}{l}\mathrm{HC}-\mathrm{N} \\
\mathrm{H}-\mathrm{CN}\end{array}$ & $\begin{array}{l}1.153 \\
1.066\end{array}$ & $\begin{array}{l}207(\mathrm{DE}) \\
114(\mathrm{DE})\end{array}$ \\
\hline $\mathrm{HgBr}_{2}$ & ${ }^{1} \sum$ & $\begin{array}{r}\mathrm{BrHg}-\mathrm{Br} \\
\mathrm{Hg}-\mathrm{Br}\end{array}$ & 2.43 & $\begin{array}{l}72(\mathrm{DE}) \\
44(\mathrm{BE})\end{array}$ \\
\hline $\mathrm{HgBrI}$ & ${ }^{1} \sum$ & $\mathrm{BrHg}-\mathrm{I}$ & & $64(\mathrm{DE})$ \\
\hline $\mathrm{HgCl}_{2}$ & ${ }^{1} \sum$ & $\begin{array}{r}\mathrm{ClHg}-\mathrm{Cl} \\
\mathrm{Hg}-\mathrm{Cl}\end{array}$ & 2.30 & $\begin{array}{l}81(\mathrm{DE}) \\
54(\mathrm{BE})\end{array}$ \\
\hline $\mathrm{HgClBr}$ & ${ }^{1} \sum$ & $\mathrm{BrHg}-\mathrm{Cl}$ & & $77(\mathrm{DE})$ \\
\hline $\mathrm{HgClI}$ & ${ }^{1} \sum$ & $\begin{array}{l}\mathrm{HHg}-\mathrm{Cl} \\
\mathrm{ClHg}-\mathrm{I}\end{array}$ & & $\begin{array}{l}75(\mathrm{DE}) \\
63(\mathrm{DE})\end{array}$ \\
\hline
\end{tabular}


Table $3-1$ (continued)

\begin{tabular}{|c|c|c|c|c|}
\hline Molecule & $\begin{array}{l}\text { Ground } \\
\text { state }\end{array}$ & Bond & $\begin{array}{l}\text { Bond } \\
\text { length, A }\end{array}$ & $\begin{array}{c}\text { Bond energies } \\
\text { kcal/mole }\end{array}$ \\
\hline $\mathrm{HgF}_{2}$ & ${ }^{1} \sum$ & $\begin{array}{r}F H g-F \\
H g-F\end{array}$ & & $\begin{array}{r}100(\mathrm{DE}) \\
66(\mathrm{BE})\end{array}$ \\
\hline $\mathrm{HgI}_{2}$ & ${ }^{1} \sum$ & $\begin{array}{r}\mathrm{IHg}-\mathrm{I} \\
\mathrm{Hg}-\mathrm{I}\end{array}$ & 2.60 & $\begin{array}{l}60(\mathrm{DE}) \\
35(\mathrm{BE})\end{array}$ \\
\hline $\mathrm{NO}_{2}{ }^{+}$ & ${ }^{1} \sum$ & $\mathrm{N}-\mathrm{C}$ & 1.10 & \\
\hline $\mathrm{MgCl}_{2}$ & ${ }^{1} \sum$ & $\begin{array}{r}\mathrm{ClMg}-\mathrm{Cl} \\
\mathrm{Mg}-\mathrm{Cl}\end{array}$ & 2.18 & $\begin{array}{r}136(\mathrm{DE}) \\
99(\mathrm{BE})\end{array}$ \\
\hline $\mathrm{SiS}_{2}$ & ${ }^{1} \sum$ & $\mathrm{Si}-\mathrm{S}$ & & $70(\mathrm{BE})$ \\
\hline $\mathrm{ZnCl}_{2}$ & ${ }^{1} \sum$ & $\mathrm{ClZn}-\mathrm{Cl}$ & 2.12 & $96(\mathrm{DE})$ \\
\hline $\mathrm{ZnI}_{2}$ & ${ }^{1} \sum$ & $I Z n-I$ & & $53(\mathrm{DE})$ \\
\hline
\end{tabular}

a Data from T. L. Cottrell, The Strengths of Chemical Bonds, Butterworths, London, 1958, Table 11.5.1.

\section{EXAMPLE}

Our purpose is to calculate the average $\mathrm{Ca}-\mathrm{Cl}$ bond energy in $\mathrm{CaCl}_{2}$ :

$$
\mathrm{Cl}_{a}{ }^{-}-\mathrm{R}-\mathrm{Ca}^{++}-\mathrm{R}-\mathrm{Cl}_{b}^{-}
$$

For $\mathrm{CaCl}_{2}$ (or any $\mathrm{MX}_{2}$ ) there are two attractions, $\mathrm{Ca}^{++}-\mathrm{Cl}_{a}{ }^{-}$and $\mathrm{Ca}^{++}-\mathrm{Cl}_{b}{ }^{-}$, each at a distance of $R$. In addition there is one repulsion, $\mathrm{Cl}_{a}{ }^{-}-\mathrm{Cl}_{b}{ }^{-}$, at a distance of $2 \mathrm{R}$. The sum of these electrostatic terms is represented

$$
\text { electrostatic energy }=-\frac{2 e^{2}}{R}-\frac{2 e^{2}}{R}+\frac{e^{2}}{2 R}=-\frac{3.5 e^{2}}{R}
$$

The energy per bond is one-half $-3.5 e^{2} / R$, or $-1.75 e^{2} / R$. The van der Waals energy can be approximated again as an inert-gas-pair interaction. In this case we have one Ar-Ar interaction for each bond. The inert-gas-pair approximation of the van der Waals energy is not expected to be as good for the $\mathrm{MX}_{2}$ molecules as for the 
MX molecules, however, owing to the small size of $\mathrm{M}^{++}$compared to that of the isoelectronic inert gas atoms (see Fig. 3-13). Thus the actual $\mathrm{Ca}^{++}-\mathrm{Cl}^{-}$van der Waals repulsion energy is probably less than that calculated.

The final expression for the energy of each $\mathrm{Ca}^{++}-\mathrm{Cl}^{-}$bond is

$$
\mathrm{PE}=\text { potential energy }=\frac{-1.75 e^{2}}{R}+b e^{-a R}-\frac{d}{R^{6}}
$$

The $\mathrm{Ca}-\mathrm{Cl}$ bond length in $\mathrm{CaCl}_{2}$ is $2.54 \mathrm{~A}$, or 4.82 au. On substituting the Ar-Ar parameters from Table 2-9, we have

$$
\mathrm{PE}=\frac{-1.75}{4.82}+350 e^{(-1.92)(4.82)}-\frac{103}{(4.82)^{6}}
$$

or

$$
\mathrm{PE}=-0.337 \mathrm{au}=-9.17 \mathrm{eV}
$$

The $9.17 \mathrm{eV}$ is one-half the energy required to dissociate $\mathrm{CaCl}_{2}$ into ions,

$$
\mathrm{CaCl}_{2} \stackrel{E^{\prime}}{\rightarrow} \mathrm{Ca}^{++}+\mathrm{Cl}^{-}+\mathrm{Cl}^{-} \quad E^{\prime}=-2 \mathrm{PE}
$$

For the average bond energy $\mathrm{BE}$, we have the process

$$
\begin{gathered}
\mathrm{CaCl}_{2} \stackrel{E}{\rightarrow} \mathrm{Ca}+\mathrm{Cl}+\mathrm{Cl} \\
E=E^{\prime}+2 \mathrm{EA}(\mathrm{Cl})-\mathrm{IP}_{1}(\mathrm{Ca})-\mathrm{IP}_{2}(\mathrm{Ca}) \text { and } \mathrm{BE}=\frac{E}{2}
\end{gathered}
$$

With $\mathrm{EA}(\mathrm{Cl})=3.61 \mathrm{eV}, \mathrm{IP}_{1}(\mathrm{Ca})=6.11 \mathrm{eV}, \mathrm{IP}_{2}(\mathrm{Ca})=11.87 \mathrm{eV}$, and $E^{\prime}=18.34 \mathrm{eV}$, we obtain $E=7.58 \mathrm{eV}$ or $175 \mathrm{kcal} / \mathrm{mole}$ and

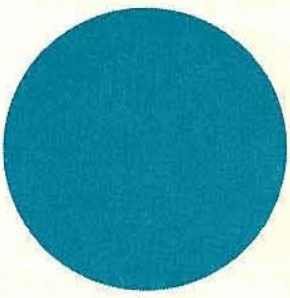

Ar

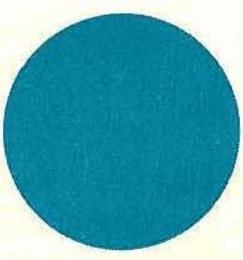

$\mathrm{K}^{+}$

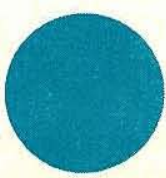

$\mathrm{Ca}^{2+}$

Figure 3-13 Relative effective sizes of $\mathrm{Ar}, \mathrm{K}^{+}$, and $\mathrm{Ca}^{2+}$. 
$\mathrm{BE}(\mathrm{Ca}-\mathrm{Cl}) \cong 88 \mathrm{kcal} / \mathrm{mole}$. This calculated value of $88 \mathrm{kcal} /$ mole may be compared with the experimental value of $113 \mathrm{kcal} /$ mole. We see that the ionic model for $\mathrm{CaCl}_{2}$ is not as good as the ionic model for the alkali halides. This is evidence that the alkaline earth halides have more "covalent character" than the alkali halides. Thus, it is likely that there are important covalent-bond contributions to the bond energy of $\mathrm{CaCl}_{2}$.

Experimental bond energies for a number of alkaline earth halides are given in Table 3-1.

\section{SUPPLEMENTARY PROBLEMS}

1. Work out the ground-state term for the molecule $\mathrm{N}_{3}$.

2. Calculate the $\mathrm{Be}-\mathrm{Cl}$ bond energy in $\mathrm{BeCl}_{2}$. The value of $\mathrm{IP}_{2}(\mathrm{Be})$ is $18.21 \mathrm{eV}$.

3. Discuss the bonding in $\mathrm{CO}_{2}, \mathrm{CS}_{2}$, and $\mathrm{CSe}_{2}$ in terms of $\mathrm{MO}$ theory. Compare the bond properties of these molecules. 


\section{IV}

\section{Trigonal-Planar Molecules}

\section{4-1 $\mathrm{BF}_{3}$}

$\mathrm{B}$ oron trifluoride has a trigonal-planar structure, with all $\mathrm{F}-\mathrm{B}-\mathrm{F}$ bond angles ${ }^{1} 120^{\circ}$. Boron has $2 s$ and $2 p$ orbitals that bond with the fluorine $2 s$ and $2 p$ orbitals. A convenient coordinate system for a discussion of bonding in $\mathrm{BF}_{3}$ is shown in Fig. 4-1.

We need only one $\sigma$ valence orbital from each fluorine. We shall use in the discussion only the $2 p$ orbital, since the molecular orbitals derived are appropriate for any combination of $2 s$ and $2 p$. However, it is probable that the very stable fluorine $2 s$ orbital is not appreciably involved in the $\sigma$ bonding. The ionization potential of an electron in the $2 s$ orbital of fluorine is over $40 \mathrm{eV}$.

\section{4-2 $\sigma$ MOLECULAR ORBITALS}

The $\sigma$ molecular orbitals are formed using the $2 s, 2 p_{x}$, and $2 p_{y}$ boron orbitals, along with the $2 p_{z_{a}}, 2 p_{z_{b}}$, and $2 p_{z_{c}}$ orbitals of the fluorine atoms. We must find the linear combinations of $2 p_{z_{a}}, 2 p_{z_{b}}$, and $2 p_{z_{c}}$ that give maximum overlap with $2 s, 2 p_{x}$, and $2 p_{y}$. The

\footnotetext{
${ }^{1}$ Bond angle is a commonly used term, meaning the angle between "internuclear lines." 

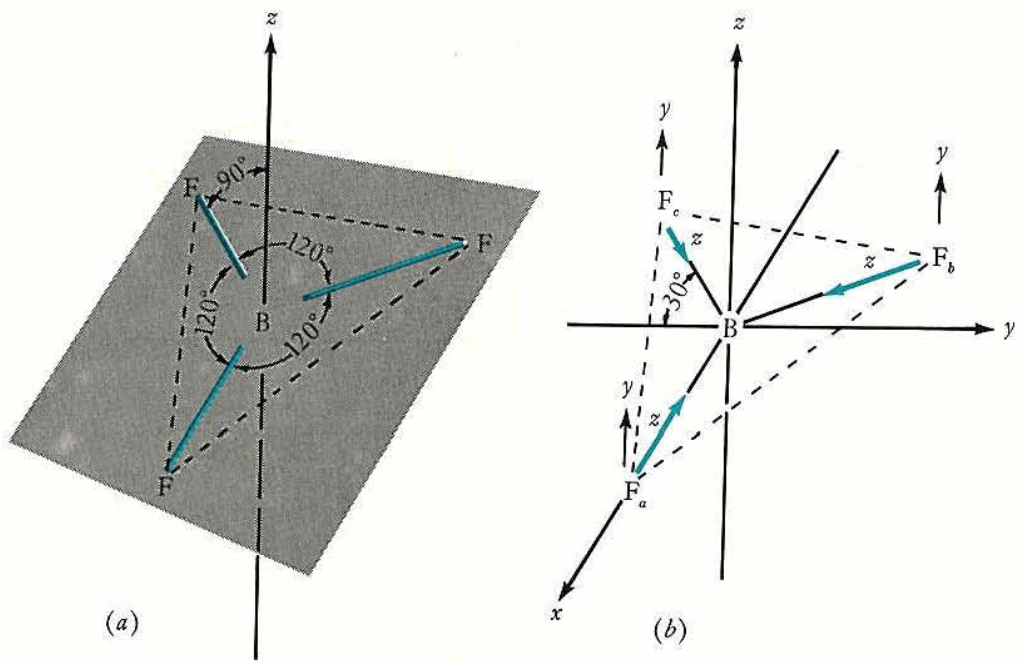

Figure 4-1 Coordinate system for $\mathbf{B F}_{3}$.

boron $2 s$ orbital is shown in Fig. 4-2. The combination $\left(2 p_{z_{a}}+\right.$ $\left.2 p_{z_{b}}+2 p_{z_{c}}\right)$ overlaps the $2 s$ orbital. Thus the molecular orbitals derived from the boron $2 s$ orbital are (using the shorthand $z_{a}=2 p_{z_{a}}$, $z_{b}=2 p_{z_{b}}$, and $\left.z_{c}=2 p_{z_{c}}\right)$ :

$$
\begin{aligned}
& \psi\left(\sigma_{s}^{b}\right)=C_{1} 2 s+C_{2}\left(z_{a}+z_{b}+z_{c}\right) \\
& \psi\left(\sigma_{s}^{*}\right)=C_{3} 2 s-C_{4}\left(z_{a}+z_{b}+z_{c}\right)
\end{aligned}
$$

The boron $2 p_{y}$ orbital is shown in Fig. 4-3. The combination $\left(z_{b}-z_{c}\right)$ matches the positive and negative lobes of $2 p_{y}$. The molecular orbitals from $2 p_{y}$ are:

$$
\begin{aligned}
& \psi\left(\sigma_{y}^{b}\right)=C_{5} 2 p_{y}+C_{6}\left(z_{b}-z_{c}\right) \\
& \psi\left(\sigma_{y}{ }^{*}\right)=C_{7} 2 p_{y}-C_{8}\left(z_{b}-z_{c}\right)
\end{aligned}
$$

The boron $2 p_{x}$ orbital is shown in Fig. 4-4. A combination $\left(z_{a}-z_{b}-z_{c}\right)$ correctly overlaps the lobes of $2 p_{x}$. There is a minor complication, however: the overlaps of $z_{a}, z_{b}$, and $z_{c}$ with $2 p_{x}$ are not the same. Specifically, $z_{a}$ points directly at the positive lobe of $2 p_{x}$, 


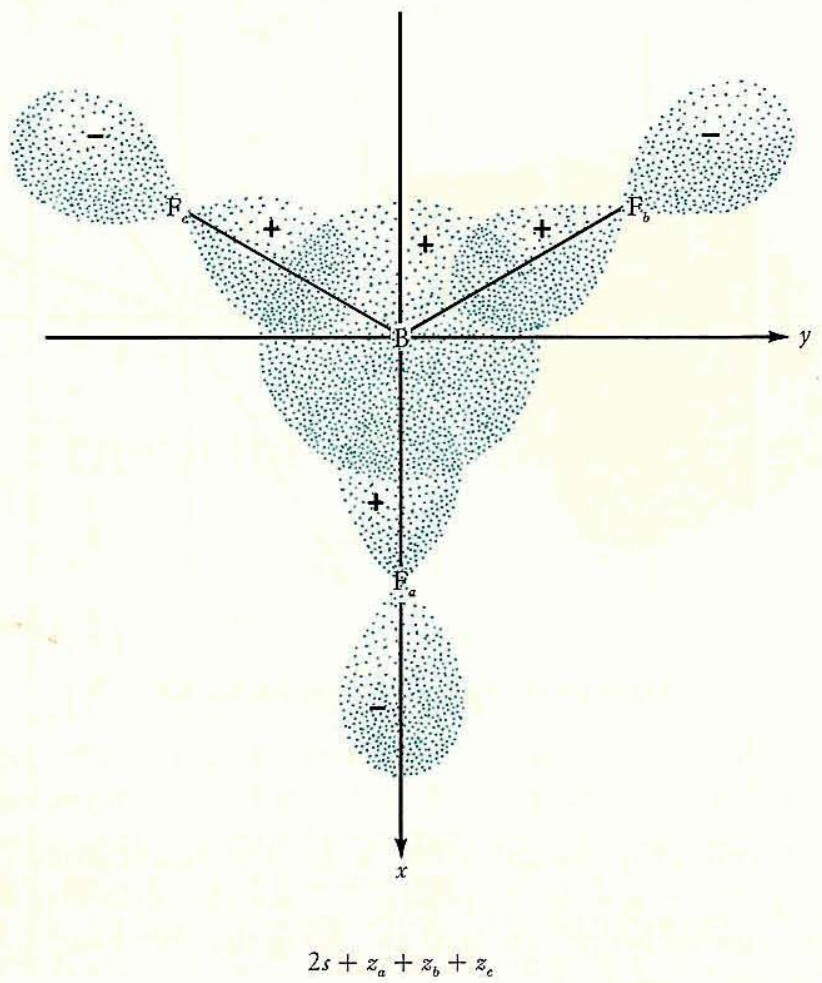

Figure 4-2 Overlap of the boron $2 s$ orbital with the $2 p_{z}$ orbitals of the fluorine atoms.

whereas $z_{b}$ and $z_{c}$ are $60^{\circ}$ displaced from a comparable overlap with the negative lobe. In order to relate $z_{a}$ to $z_{b}$ and $z_{c}$, we must find the fraction of $2 p_{x}$ that can be resolved along the $z_{b}$ line. This fraction is simply $\cos 60^{\circ}$, or $\frac{1}{2}$. We deduce that the sum $z_{b}+z_{c}$ gives the same overlap with $2 p_{x}$ as $z_{a}$ does alone. Then the proper combination is $\left(z_{a}-\frac{1}{2} z_{b}-\frac{1}{2} z_{c}\right)$, and the $\sigma$ molecular orbitals from $2 p_{x}$ are:

$$
\begin{aligned}
& \psi\left(\sigma_{x}^{b}\right)=C_{9} 2 p_{x}+C_{10}\left(z_{a}-\frac{1}{2} z_{b}-\frac{1}{2} z_{c}\right) \\
& \psi\left(\sigma_{x}^{*}\right)=C_{11} 2 p_{x}-C_{12}\left(z_{a}-\frac{1}{2} z_{b}-\frac{1}{2} z_{c}\right)
\end{aligned}
$$




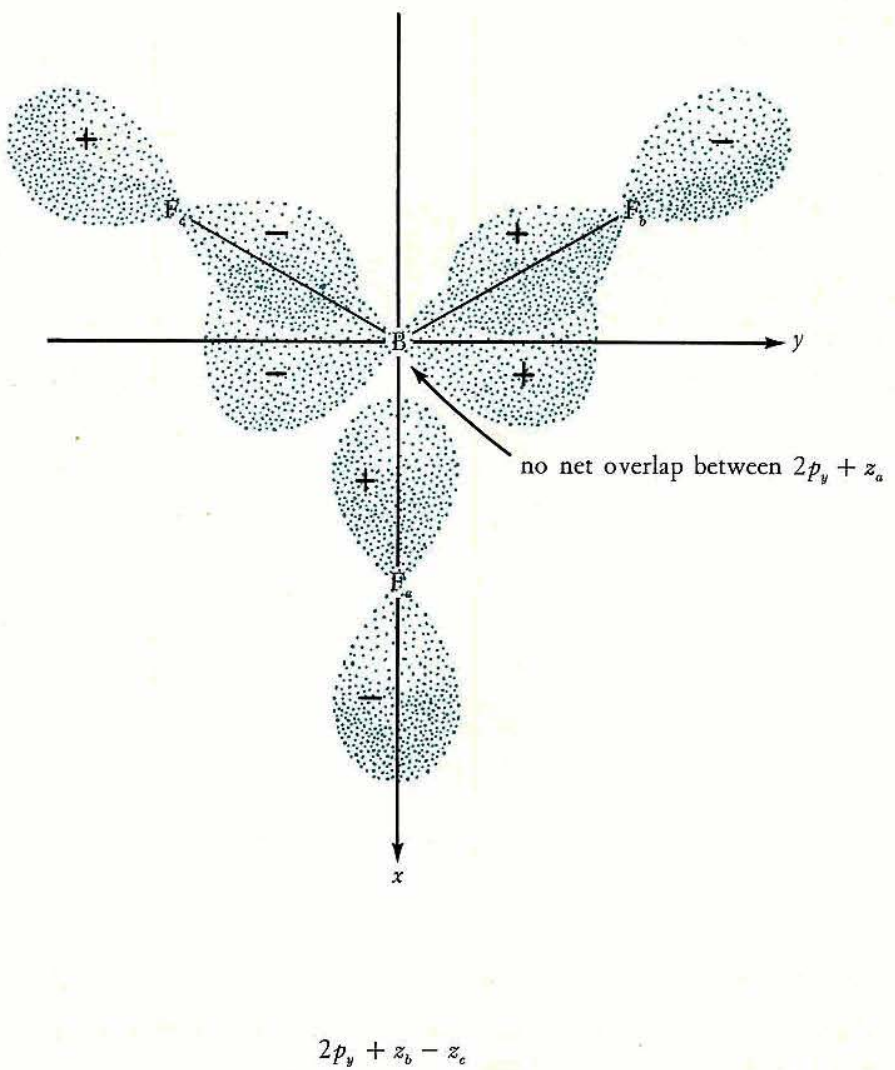

Figure 4-3 Overlap of the boron $2 p_{y}$ orbital with the $2 p_{z}$ orbitals of the fluorine atoms.

\section{4-3 $\pi$ MOLECULAR ORBITALS}

The $\pi$ molecular orbitals are formed using the boron $2 p_{z}$ orbital and the $2 p_{y}$ orbitals of the fluorine atoms. The combination $\left(y_{a}+y_{b}+\right.$ $y_{c}$ ) matches the $2 p_{z}$ orbital, as shown in Fig. 4-5. Thus the bonding and antibonding $\pi$ molecular orbitals are:

$$
\begin{aligned}
& \psi\left(\pi_{z}^{b}\right)=C_{13} 2 p_{z}+C_{14}\left(y_{a}+y_{b}+y_{c}\right) \\
& \psi\left(\pi_{z}^{*}\right)=C_{15} 2 p_{z}-C_{16}\left(y_{a}+y_{b}+y_{c}\right)
\end{aligned}
$$




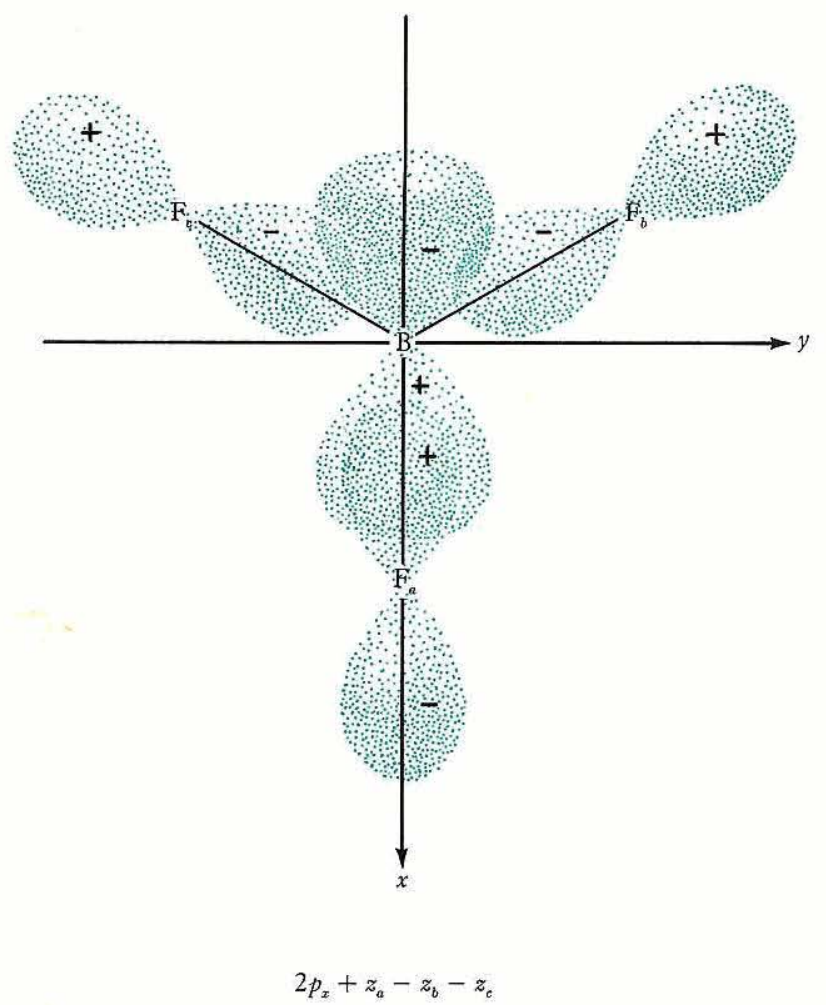

Figure 4-4 Overlap of the boron $2 p_{x}$ orbital with the $2 p_{z}$ orbitals of the fluorine atoms.

Since we started with three fluorine $2 p_{y}$ orbitals, there are two more independent linear combinations of $y_{a}, y_{b}$, and $y_{c}$. One satisfactory pair is $\left(y_{a}-y_{c}\right)$ and $\left(y_{a}-2 y_{b}+y_{c}\right)$. As shown in Fig. 4-6, these orbital combinations do not overlap the boron $2 p_{z}$ orbital. Thus they are nonbonding in $\mathrm{BF}_{3}$, and we have

$$
\begin{gathered}
\psi\left(\pi_{1}\right)=\frac{1}{\sqrt{2}}\left(y_{a}-y_{c}\right) \\
\psi\left(\pi_{2}\right)=\frac{1}{\sqrt{6}}\left(y_{a}-2 y_{b}+y_{c}\right)
\end{gathered}
$$



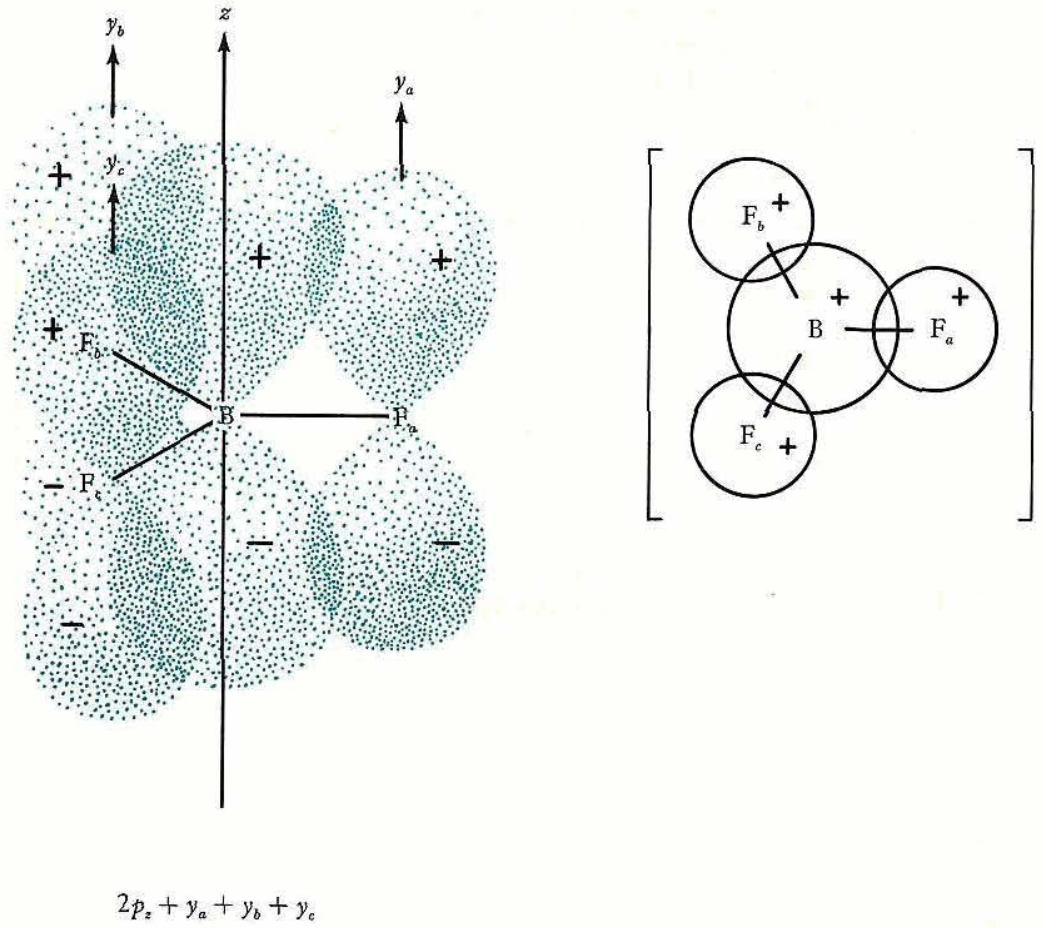

Figure 4-5 Overlap of the boron $2 p_{z}$ orbital with the $2 p_{y}$ orbitals of the fluorine atoms.

\section{4-4 ENERGY LEVELS FOR $\mathrm{BF}_{3}$}

The molecular-orbital energy-level scheme for $\mathrm{BF}_{3}$ is shown in Fig. 4-7. The fluorine valence orbitals are more stable than the boron valence orbitals, and so electrons in bonding molecular orbitals spend more time in the domain of the fluorine nuclei. The $\sigma_{x}$ and $\sigma_{y}$ molecular orbitals are degenerate in trigonal-planar molecules such as $\mathrm{BF}_{3}$. Since this is by no means obvious from Eqs. (4-3), $(4-4),(4-5)$, and (4-6), we shall devote a short section to an explanation. 


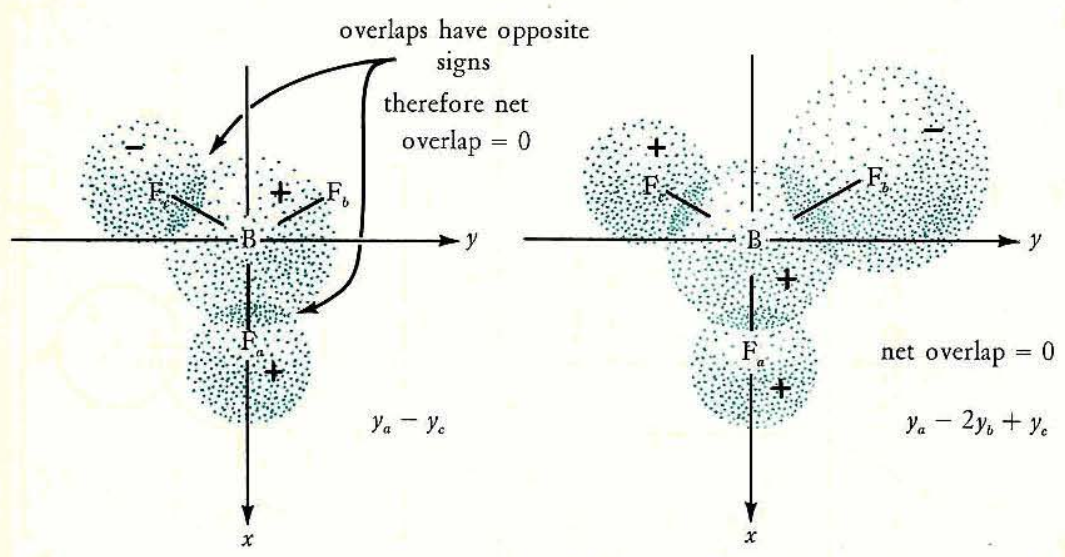

Figure 4-6 Two combinations of the fluorine $2 p_{y}$ orbitals that have zero overlap with the boron $2 p_{z}$ orbital.

\section{4-5 EQUIVALENCE OF $\sigma_{x}$ AND $\sigma_{y}$ ORBITALS}

The total overlap of the normalized combination $\sqrt{\frac{2}{3}}\left(z_{a}-\frac{1}{2} z_{b}-\right.$ $\left.\frac{1}{2} z_{c}\right)$ with $2 p_{x}$ will be called $S\left(\sigma_{x}\right)$; the total overlap of $(1 / \sqrt{2})$ $\left(z_{b}-z_{c}\right)$ with $2 p_{y}$ will be called $S\left(\sigma_{y}\right)$. A direct $\sigma$ overlap, such as the overlap between $z_{a}$ and $2 p_{x}$ (Fig. $4-8$ ), will be called $S\left(p_{\sigma}, p_{\sigma}\right)$. To evaluate $S\left(\sigma_{x}\right)$ and $S\left(\sigma_{y}\right)$ in terms of $S\left(p_{\sigma}, p_{\sigma}\right)$, we use the following calculations:

$$
\begin{aligned}
S\left(\sigma_{x}\right) & =\sqrt{\frac{2}{3}} S\left(2 p_{x}\right)\left(z_{a}-\frac{1}{2} z_{b}-\frac{1}{2} z_{c}\right) d \tau \\
& =\sqrt{\frac{2}{3}}\left[S\left(p_{\sigma}, p_{\sigma}\right)+\frac{1}{2} \cos 60^{\circ} S\left(p_{\sigma}, p_{\sigma}\right)+\frac{1}{2} \cos 60^{\circ} S\left(p_{\sigma}, p_{\sigma}\right)\right] \\
& =\sqrt{\frac{2}{3}}\left(\frac{3}{2}\right)\left[S\left(p_{\sigma}, p_{\sigma}\right)\right]=\sqrt{\frac{3}{2}} S\left(p_{\sigma}, p_{\sigma}\right) \\
S\left(\sigma_{y}\right) & =\frac{1}{\sqrt{2}} \mathcal{S}\left(2 p_{y}\right)\left(z_{b}-z_{c}\right) d \tau \\
& =\frac{1}{\sqrt{2}}\left[\cos 30^{\circ} S\left(p_{\sigma}, p_{\sigma}\right)+\cos 30^{\circ} S\left(p_{\sigma}, p_{\sigma}\right)\right] \\
& =\frac{1}{\sqrt{2}}\left(\frac{\sqrt{3}}{2}+\frac{\sqrt{3}}{2}\right)\left[S\left(p_{\sigma}, p_{\sigma}\right)\right]=\sqrt{\frac{3}{2}} S\left(p_{\sigma}, p_{\sigma}\right)
\end{aligned}
$$

Since the overlaps are the same in $\sigma_{x}$ and $\sigma_{y}$, and since the com- 


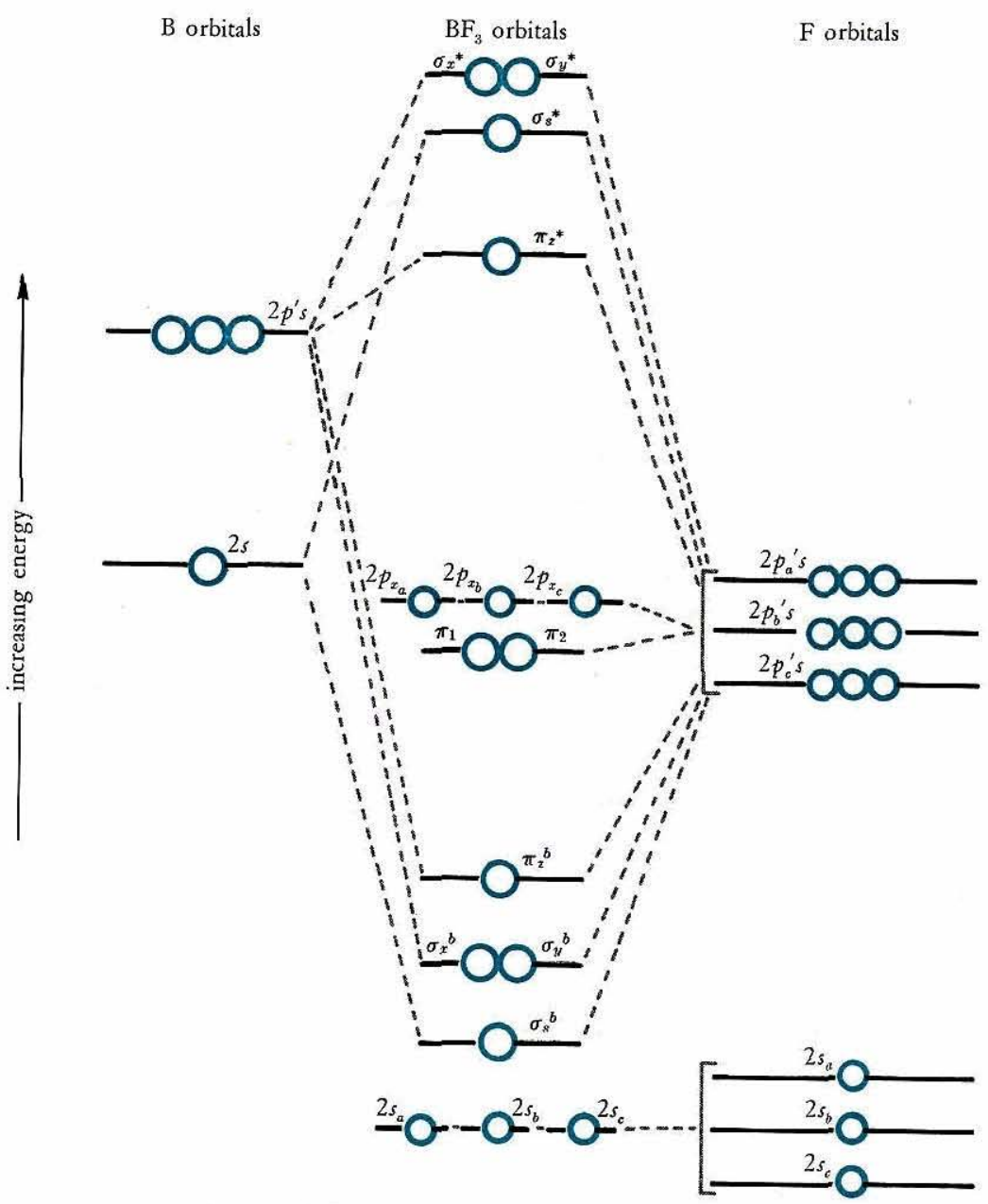

Figure 4-7 Relative orbital energies in $\mathrm{BF}_{3}$.

bining boron and fluorine valence orbitals have the same initial energies, it follows that $\sigma_{x}$ and $\sigma_{y}$ are degenerate in trigonal-planar molecules. However, it is worth pointing out that $\sigma_{x}$ and $\sigma_{y}$ are not necessarily degenerate if the bond angles deviate from $120^{\circ}$. 


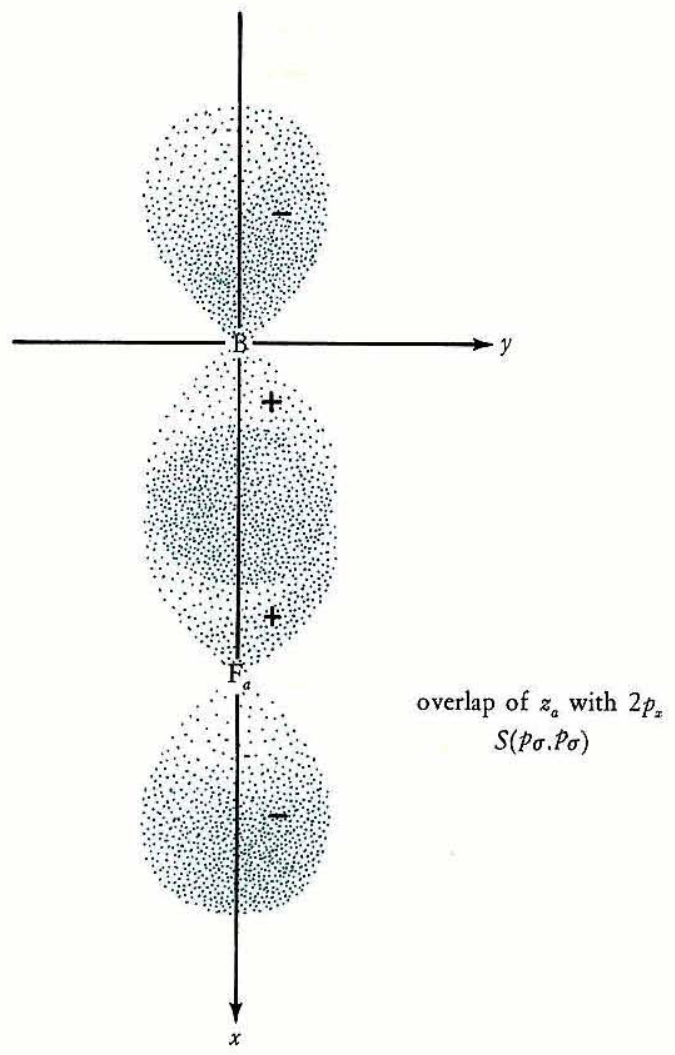

Figure 4-8 Standard two-atom $\sigma$ overlap between $p$ orbitals.

4-6 GROUND STATE OF $\mathrm{BF}_{3}$

There are 24 valence electrons in $\mathrm{BF}_{3}$ [ 7 from each fluorine $\left(2 s^{2} 2 p^{5}\right)$, 3 from the boron $\left(2 s^{2} 2 p\right)$ ]. Placing these electrons in the most stable molecular orbitals, we obtain a ground-state configuration:

$$
\begin{aligned}
\left(2 s_{a}\right)^{2}\left(2 s_{b}\right)^{2}\left(2 s_{c}\right)^{2}\left(\sigma_{s}^{b}\right)^{2}\left(\sigma_{x}^{b}\right)^{2}\left(\sigma_{y}{ }^{b}\right)^{2}\left(\pi_{z}^{b}\right)^{2}\left(\pi_{1}\right)^{2}\left(\pi_{2}\right)^{2}\left(2 p_{x_{a}}\right)^{2}\left(2 p_{x_{b}}\right)^{2}\left(2 p_{x_{c}}\right)^{2} & \\
S & =0
\end{aligned}
$$

There are six electrons in $\sigma^{b}$ orbitals to give a total of three $\sigma$ bonds for $\mathrm{BF}_{3}$; in addition, the two electrons the $\pi_{z}^{b}$ orbital indicate one $\pi$ 
bond. The $\mathrm{B}-\mathrm{F}$ bond length in $\mathrm{BF}_{3}$ is $1.291 \mathrm{~A}$; the $\mathrm{B}-\mathrm{F}$ bond energy is $154 \mathrm{kcal} / \mathrm{mole}$.

\section{4-7 VALENCE BONDS FOR $\mathrm{BF}_{3}$}

The valence-bond description of the ground state of $\mathrm{BF}_{3}$ is comparable to the molecular-orbital description. Three equivalent $s p^{2}$ hybrid orbitals are formed first by mixing together the $2 s, 2 p_{x}$, and $2 p_{y}$ boron orbitals, as shown in Fig. 4-9. Each $s p^{2}$ hybrid orbital has one-third $s$ and two-thirds $p$ character. These three $s p^{2}$ orbitals are then used to make three electron-pair $\sigma$ bonds with the $2 p_{z}$ fluorine orbitals. In addition, the $2 p_{z}$ boron orbital can be used to make a $\pi$ bond with any one of the three fluorine $2 p_{y}$ orbitals. Thus there are three equivalent resonance structures for $\mathrm{BF}_{3}$, as shown in Fig. 4-10. Notice that the three resonance structures move the electron-pair $\pi$ bond around the "ring"; this is analogous to having two electrons in the delocalized $\pi_{z}^{b}$ moleculat orbital.

\section{PROBLEM}

$4-7$. Construct the wave functions for the three equivalent $s p^{2}$ hybrid orbitals.

Solution. It is convenient to use the coordinate system shown in Fig. 4-1, directing the three $s p^{2}$ hybrid orbitals at atoms $a, b$, and $c$. The $s, p_{x}$, and $p_{y}$ orbitals are used to form the $s p^{2}$ orbitals. Each hybrid orbital has one-third $s$ character. Of the two $p$ orbitals, only the $p_{x}$ is used to bond with atom $a$ ( $p_{y}$ has zero overlap with $a$ ). Since each $s p^{2}$ orbital has two-thirds $p$ character, the wave function for $s p_{a}^{2}$ is

$$
\psi\left(s p_{a}^{2}\right)=\sqrt{\frac{1}{3}} s+\sqrt{\frac{2}{3}} p_{x}
$$

The remaining third of the $p_{x}$ orbital is divided equally between. $b$ and $c$. Since the $p_{y}$ orbital has not been used as yet, and since it overlaps equally well with $b$ and $c$, we split it up between $b$ and $c$ to complete the two-thirds $p$ character in $s p_{b}{ }^{2}$ and $s p_{c}{ }^{2}$. Choosing the algebraic signs in the functions so that large and equal lobes are directed at $b$ and $c$, we have:

$$
\begin{aligned}
& \psi\left(s p_{b}^{2}\right)=\sqrt{\frac{1}{3}} s-\sqrt{\frac{1}{6}} p_{x}+\sqrt{\frac{1}{2}} p_{y} \\
& \psi\left(s p_{c}^{2}\right)=\sqrt{\frac{1}{3}} s-\sqrt{\frac{1}{6}} p_{x}-\sqrt{\frac{1}{2}} p_{y}
\end{aligned}
$$



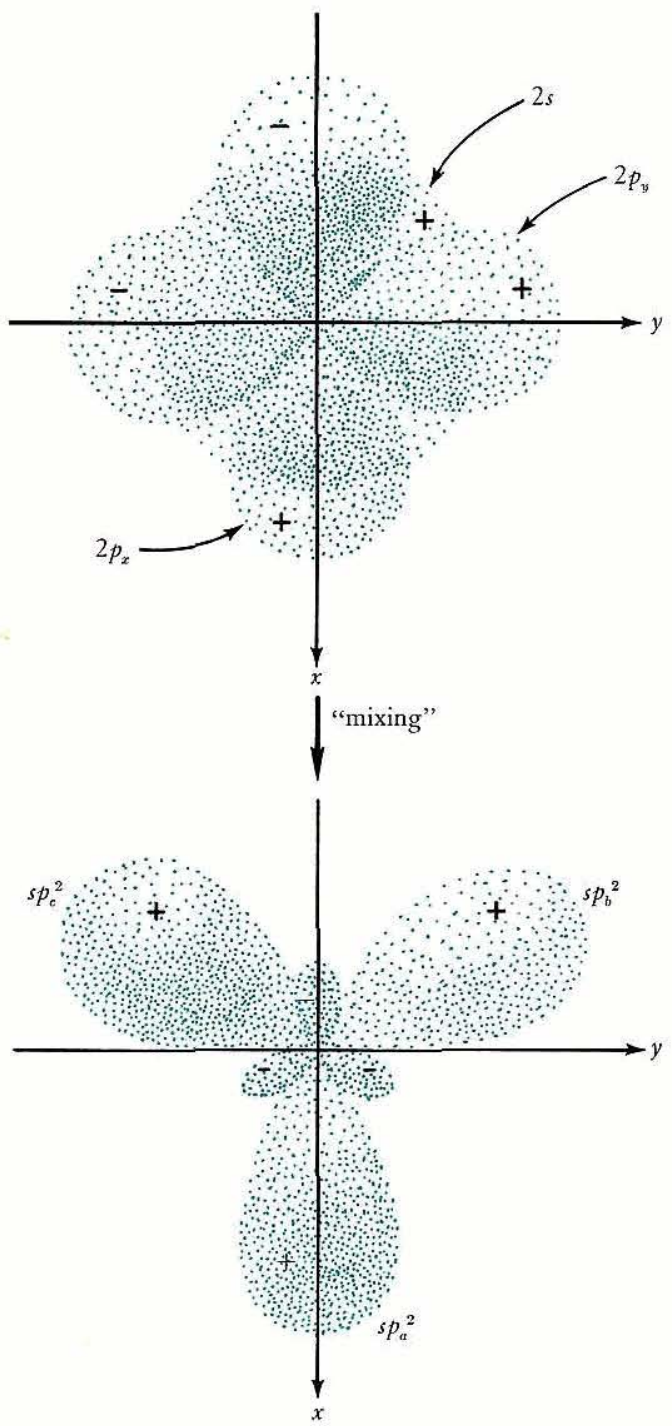

Figure 4-9 Formation of three $s p^{2}$ hybrid orbitals. 


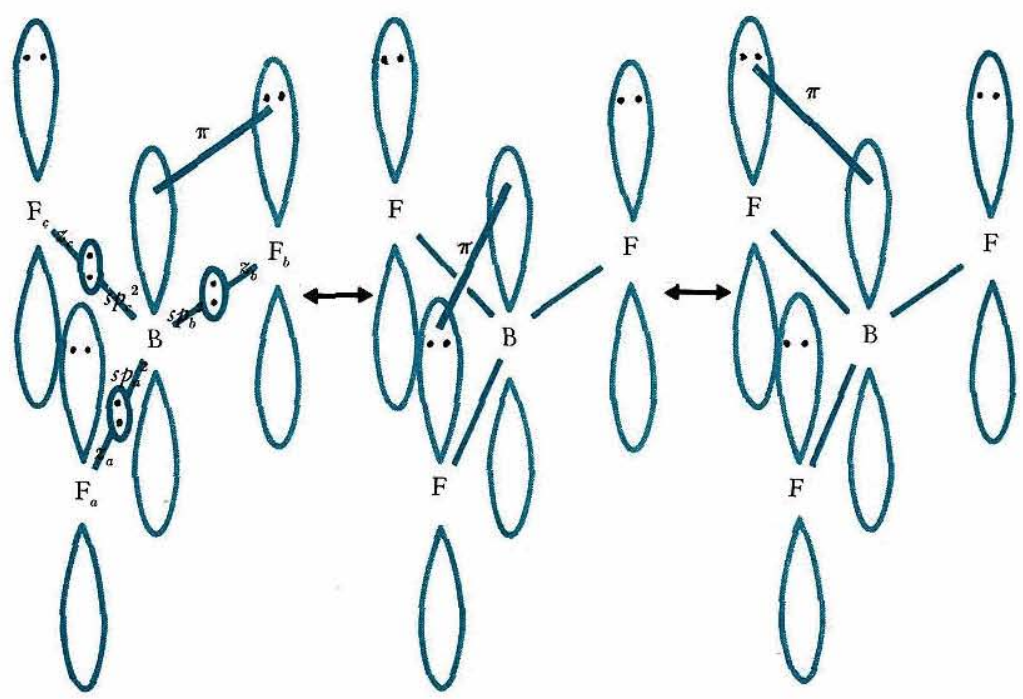

Figure 4-10 Valence-bond structures for $\mathbf{B F}_{3}$.

The boundary surfaces of $s p_{a}^{2}, s p_{b}^{2}$, and $s p_{c}^{2}$ are shown in Fig. 4-9. Bonding orbitals are combinations of the $s p^{2}$ orbitals and appropriate $\sigma$ orbitals of atoms $a, b$, and $c$ :

$$
\begin{aligned}
& \Psi_{a}=c_{1}^{\psi}\left(s p_{a}^{2}\right)+c_{2} z_{a} \\
& \Psi_{b}=c_{1}^{\psi}\left(s p_{b}^{2}\right)+c_{2} z_{b} \\
& \Psi_{c}=c_{1}^{\psi}\left(s p_{c}^{2}\right)+c_{2} z_{c}
\end{aligned}
$$

\section{4-8 OTHER TRIGONAL-PLANAR MOLECULES}

Elements in the boron family are the central atoms in many trigonal-planar molecules. Also, several important molecules and complex ions containing oxygen have trigonal-planar structures, among them $\mathrm{SO}_{3}, \mathrm{NO}_{3}{ }^{-}$, and $\mathrm{CO}_{3}{ }^{2-}$. Bond properties of a number of trigonal-planar molecules are given in Table 4-1. The $\mathrm{BH}_{3}$ molecule, which is presumably trigonal planar, is more stable in a dimeric form, 
Table $4-1$

Properties of Trigonal-Planar Molecules ${ }^{a}$

\begin{tabular}{lccc}
\hline Molecule & Bond & $\begin{array}{c}\text { Bond } \\
\text { length, } A\end{array}$ & $\begin{array}{c}\text { Bond energy (BE), } \\
\text { kcal/mole }\end{array}$ \\
\hline $\mathrm{BF}_{3}$ & $\mathrm{~B}-\mathrm{F}$ & 1.291 & 154 \\
$\mathrm{BCl}_{3}$ & $\mathrm{~B}-\mathrm{Cl}$ & 1.74 & 109 \\
$\mathrm{BBr}_{3}$ & $\mathrm{~B}-\mathrm{Br}$ & 1.87 & 90 \\
$\mathrm{BH}_{3}$ & $\mathrm{~B}-\mathrm{H}$ & & 93 \\
$\mathrm{~B}\left(\mathrm{CH}_{3}\right)_{3}$ & $\mathrm{~B}-\mathrm{C}$ & 1.56 & 89 \\
$\mathrm{Al}\left(\mathrm{CH}_{3}\right)_{3}$ & $\mathrm{Al}-\mathrm{C}$ & & 61 \\
$\mathrm{~B}(\mathrm{OR})_{3}{ }^{\mathrm{b}}$ & $\mathrm{B}-\mathrm{OR}$ & 1.38 & 128 \\
$\mathrm{SO}_{3}$ & $\mathrm{~S}-\mathrm{O}$ & 1.43 & 104 \\
$\mathrm{NO}_{3}{ }^{-}$ & $\mathrm{N}-\mathrm{O}$ & 1.22 & \\
$\mathrm{CO}_{3}{ }^{2-}$ & $\mathrm{C}-\mathrm{O}$ & 1.29 & \\
$\mathrm{BO}_{3}{ }^{3-}$ & $\mathrm{B}-\mathrm{O}$ & 1.38 & \\
\hline
\end{tabular}

a Data from T. L. Cottrell, The Strengths of Chemical Bonds; Butterworths, London, 1958, Table 11.5.1.

${ }^{b} \mathrm{R}=\mathrm{CH}_{3}$ or $\mathrm{C}_{2} \mathrm{H}_{5} ; \mathrm{R}=\mathrm{H}, 1.36 \mathrm{~A}$.

$$
\mathrm{BH}_{3}+\mathrm{BH}_{3} \rightarrow \mathrm{B}_{2} \mathrm{H}_{6}
$$

The bonding in diborane $\mathrm{B}_{2} \mathrm{H}_{6}$ is described in a number of other sources. $^{1}$

The $\mathrm{B}\left(\mathrm{CH}_{3}\right)_{3}$ and $\mathrm{Al}\left(\mathrm{CH}_{3}\right)_{3}$ molecules have trigonal-planar parts.

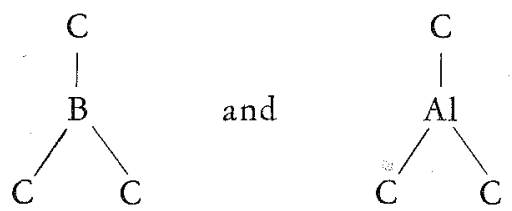

The structure around each carbon is tetrahedral, as will be described in Chapter $\mathrm{V}$.

1 See, for example, F. A. Cotton and G. Wilkinson, Advanced Inorganic Cbemistry, Wiley-Interscience, New York, 1962, pp. 200-203; W. N. Lipscomb, Boron Hydrides, Benjamin, New York, 1963, Chap. 2; C. J. Ballhausen and H. B. Gray, Introductory Notes on Molecular-Orbital Theory, Benjamin, New York, 1965, Chap. 7. 


\section{SUPPLEMENTARY PROBLEMS}

1. In most cases it is convenient to have a normalized linear combination of orbitals to bond with a central atom. For example, the combination appropriate for $2 s$ in a trigonal-planar molecule is $\left(z_{a}+z_{b}+z_{c}\right)$. The normalized combination is $\frac{1}{\sqrt{3}}\left(z_{a}+z_{b}+z_{c}\right)$.

Normalize the combinations $\left(z_{b}-z_{c}\right)$ and $\left(z_{a}-\frac{1}{2} z_{b}-\frac{1}{2} z_{c}\right)$.

2. Show that the molecular-orbital and valence-bond descriptions of $\sigma$ bonding in a trigonal-planar molecule are equivalent, if, in Eqs. (4-1), (4-3), and (4-5), $C_{1}=C_{5}=C_{9}$ and $C=\sqrt{3} C_{2}=\sqrt{2} C_{6}=$ $\sqrt{\frac{3}{2}} C_{10}$. In general, do you expect that $C_{1}=C_{5}$ ? $C_{5}=C_{9}$ ? $\sqrt{3} C_{2}=$ $\sqrt{2} C_{6}$ ? $\sqrt{2} C_{6}=\sqrt{\frac{3}{2}} C_{10}$ ? Explain. 


\section{V \\ Tetrahedral Molecules}

5-1 $\mathrm{CH}_{4}$

The methane molecule, $\mathrm{CH}_{4}$, has a tetrahedral structure. This 1 structure is shown in Fig. 5-1. With the carbon in the center of the cube, the hydrogens are then placed at opposite corners of the cube, as defined by a regular tetrahedron. The origin of the rectangular coordinate system is chosen at the center of the cube, with the $x, y$, and $z$ axes perpendicular to the faces. All the carbon valence orbitals, $2 s, 2 p_{x}, 2 p_{y}$, and $2 p_{z}$, must be used to form an adequate set of $\sigma$ molecular orbitals.

The overlap of the four $1 s$ hydrogen orbitals with the carbon $2 s$ orbital is shown in Fig. 5-2. The linear combination $\left(1 s_{a}+1 s_{b}+\right.$ $1 s_{c}+1 s_{d}$ ) is appropriate. The bonding and antibonding molecular orbitals are:

$$
\begin{aligned}
& \psi\left(\sigma_{s}^{b}\right)=C_{1} 2 s+C_{2}\left(1 s_{a}+1 s_{b}+1 s_{c}+1 s_{d}\right) \\
& \psi\left(\sigma_{s}^{*}\right)=C_{3} 2 s-C_{4}\left(1 s_{a}+1 s_{b}+1 s_{c}+1 s_{d}\right)
\end{aligned}
$$

The overlap of the four $1 s$ orbitals with the carbon $2 p$ orbital is shown in Fig. 5-3. Hydrogen orbitals $1 s_{a}$ and $1 s_{b}$ overlap the plus lobe, and orbitals $1 s_{c}$ and $1 s_{d}$ overlap the minus lobe. Thus the proper combination is $\left(1 s_{a}+1 s_{b}-1 s_{c}-1 s_{d}\right)$. 


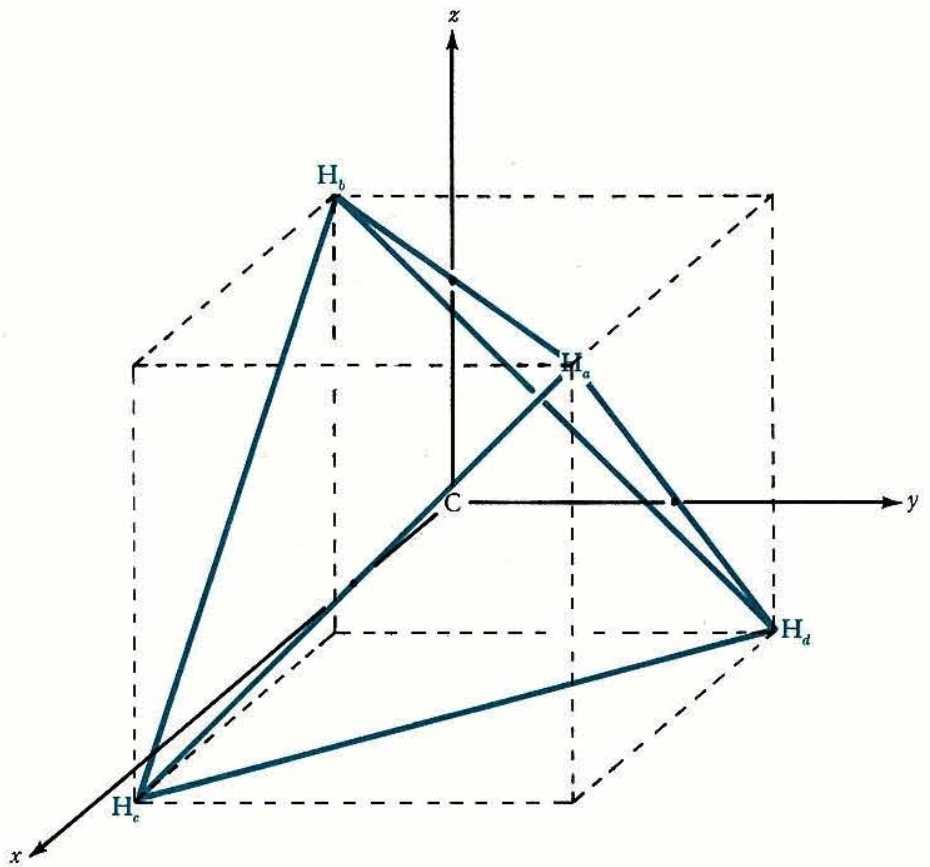

Figure 5-1 Coordinate system for $\mathrm{CH}_{4}$.

The $2 p_{x}$ and $2 p_{y}$ carbon orbitals overlap the four hydrogen orbitals in the same way as $2 p_{z}$. This is shown in Fig. 5-4. The linear combinations are $\left(1 s_{a}+1 s_{d}-1 s_{b}-1 s_{c}\right)$ with $2 p_{y}$, and $\left(1 s_{a}+1 s_{c}-\right.$ $1 s_{b}-1 s_{d}$ ) with $2 p_{x}$. The molecular orbitals are given below.

$$
\begin{aligned}
& \psi\left(\sigma_{z}{ }^{b}\right)=C_{5} 2 p_{z}+C_{6}\left(1 s_{a}+1 s_{b}-1 s_{c}-1 s_{d}\right) \\
& \psi\left(\sigma_{z}{ }^{*}\right)=C_{7} 2 p_{z}-C_{8}\left(1 s_{a}+1 s_{b}-1 s_{c}-1 s_{d}\right) \\
& \psi\left(\sigma_{y}{ }^{b}\right)=C_{9} 2 p_{y}+C_{10}\left(1 s_{a}+1 s_{d}-1 s_{b}-1 s_{c}\right) \\
& \psi\left(\sigma_{y}{ }^{*}\right)=C_{11} 2 p_{y}-C_{12}\left(1 s_{a}+1 s_{d}-1 s_{b}-1 s_{c}\right) \\
& \psi\left(\sigma_{x}{ }^{b}\right)=C_{13} 2 p_{x}+C_{14}\left(1 s_{a}+1 s_{c}-1 s_{b}-1 s_{d}\right) \\
& \psi\left(\sigma_{x}{ }^{*}\right)=C_{15} 2 p_{x}-C_{16}\left(1 s_{a}+1 s_{c}-1 s_{b}-1 s_{d}\right)
\end{aligned}
$$




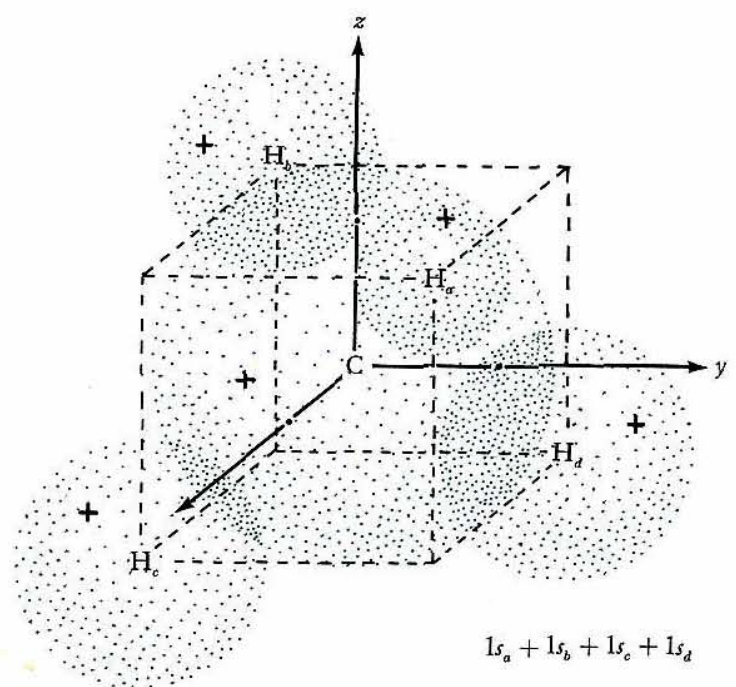

Figure 5-2 Overlap of the carbon $2 s$ orbital with the $1 s$ orbitals of the hydrogen atoms.

\section{5-2 GROUND STATE OF $\mathrm{CH}_{4}$}

The molecular-orbital energy-level scheme for $\mathrm{CH}_{4}$ is shown in Fig. 5-5. The $\sigma_{x}, \sigma_{y}$, and $\sigma_{z}$ orbitals have the same overlap in a tetrahedral molecule and are degenerate in energy. This is clear from the overlaps shown in Figs. 5-3 and 5-4.

There are eight valence electrons in $\mathrm{CH}_{4}$ because carbon is $2 s^{2} 2 p^{2}$ and each of the four hydrogens contributes a $1 s$ electron. Thus the ground state is

$$
\left(\sigma_{s}^{b}\right)^{2}\left(\sigma_{x}^{b}\right)^{2}\left(\sigma_{y}^{b}\right)^{2}\left(\sigma_{z}^{b}\right)^{2} \quad S=0
$$

There are four $\sigma$ bonds in $\mathrm{CH}_{4}$. The average $\mathrm{C}-\mathrm{H}$ bond energy is $99.3 \mathrm{kcal} / \mathrm{mole}$. The $\mathrm{C}-\mathrm{H}$ bond length in $\mathrm{CH}_{4}$ is $1.093 \mathrm{~A}$.

\section{5-3 THE TETRAHEDRAL ANGLE}

The $\mathrm{H}-\mathrm{C}-\mathrm{H}$ bond angle in $\mathrm{CH}_{4}$ is $109^{\circ} 28^{\prime}$. We can calculate the tetrahedral angle by simple trigonometry. First, we place the $\mathrm{CH}_{4}$ 


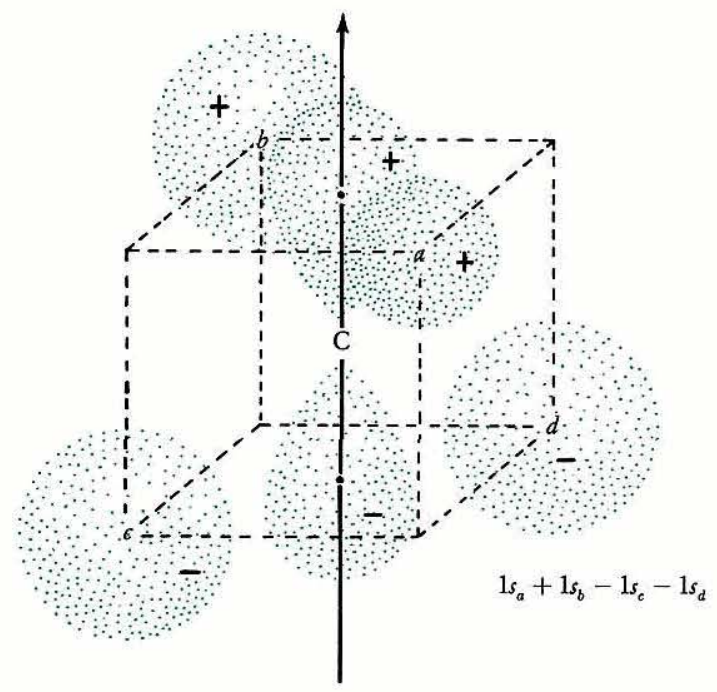

Figure 5-3 Overlap of the carbon $2 p_{z}$ orbital with the 1 s orbitals of the hydrogen atoms.

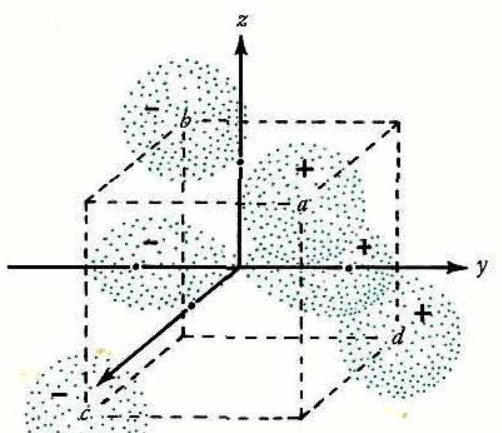

$$
1 s_{a}+1 s_{d}-1 s_{b}-1 s_{c}
$$

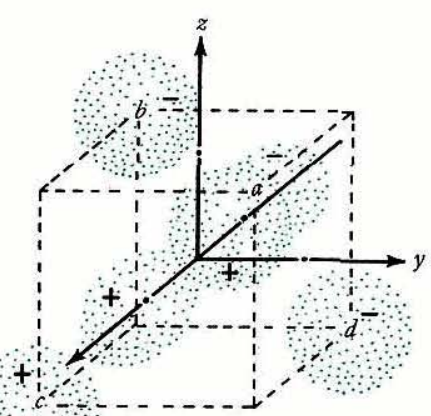

$$
1 s_{a}+1 s_{c}-1 s_{b}-1 s_{d}
$$

Figure 5-4 Overlap of the carbon $2 p_{x}$ and $2 p_{y}$ orbitals with the $1 s$ orbitals of the hydrogen atoms. 

C orbitals
$\mathrm{CH}_{4}$ orbitals
$\mathrm{H}$ orbitals

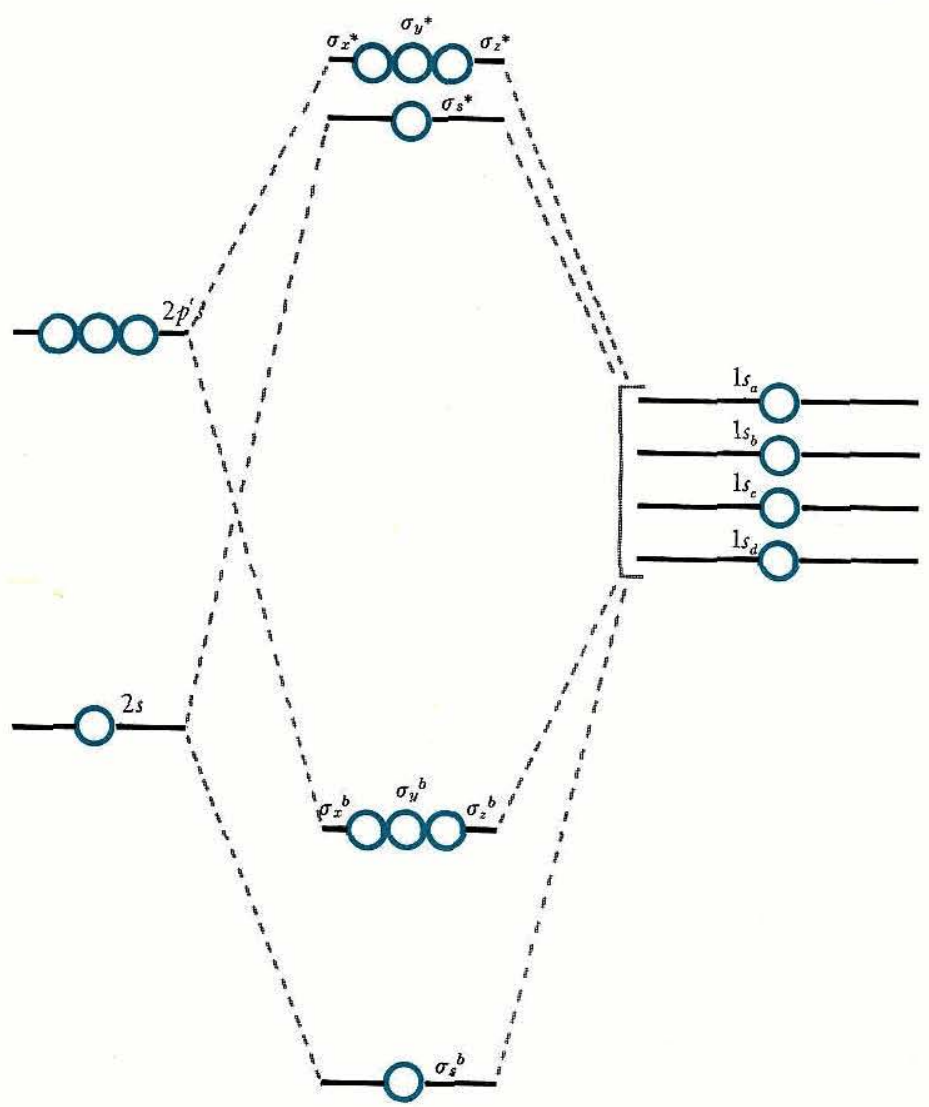

Figure 5-5 Relative orbital energies in $\mathbf{C H}_{4}$.

molecule in a unit cube, as shown in Fig. 5-6. The lengths of the sides defining the $\mathrm{H}_{a}-\mathrm{C}-\mathrm{H}_{d}$ angle $\theta$ are obtained by using the Pythagorean theorem. Thus we have the result

$$
\cos \frac{\theta}{2}=\frac{\sqrt{3}}{3} \quad \text { or } \quad \theta=109^{\circ} 28^{\prime}
$$




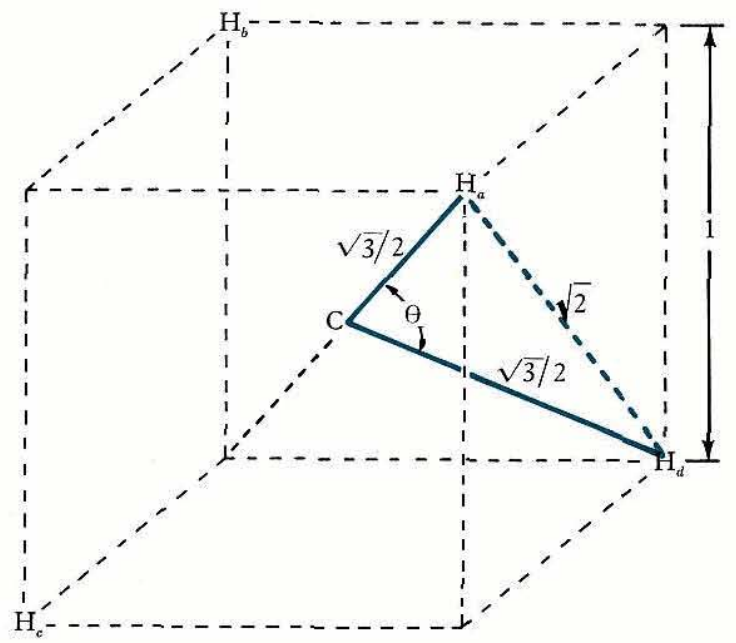

Figure 5-6 Unit-cube model for evaluating the tetrahedral angle.

\section{5-4 VALENCE BONDS FOR $\mathrm{CH}_{4}$}

Four equivalent valence orbitals centered on carbon can be constructed by scrambling together the $2 s, 2 p_{x}, 2 p_{y}$, and $2 p_{z}$ orbitals. These equivalent orbitals are called $s p^{3}$ hybrids, and their construction is shown schematically in Fig. 5-7. Each $s p^{3}$ hybrid orbital has one-fourth $s$ character and three-fourths $p$ character.

The four $s p^{3}$ orbitals are directed toward the corners of a regular tetrahedron, and thus are ideally suited for forming four localized bonding orbitals with the four hydrogen $1 s$ orbitals. The valencebond structure for $\mathrm{CH}_{4}$ is shown in Fig. 5-8.

\section{PROBLEM}

$5-1$. The normalized wave functions for the four equivalent $s p^{3}$ hybrid orbitals are listed below (coordinate system as shown in Fig. 5-7):

$$
\begin{aligned}
& \psi\left(s p_{a}^{3}\right)=\frac{1}{2} s+\sqrt{\frac{3}{4}}\left(p_{x}+p_{y}+p_{z}\right) \\
& \psi\left(s p_{b}^{3}\right)=\frac{1}{2} s+\sqrt{\frac{3}{4}}\left(-p_{x}-p_{y}+p_{z}\right)
\end{aligned}
$$



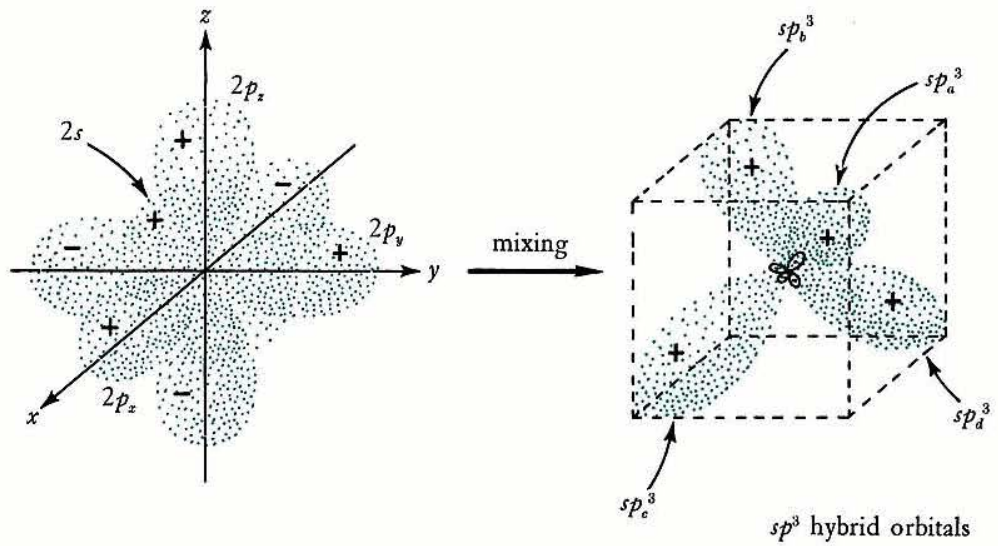

Figure 5-7 Formation of four $s p^{3}$ hybrid orbitals.

$$
\begin{aligned}
& \psi\left(s p_{c}^{3}\right)=\frac{1}{2} s+\sqrt{\frac{3}{4}}\left(p_{x}-p_{y}-p_{z}\right) \\
& \psi\left(s p_{d}^{3}\right)=\frac{1}{2} s+\sqrt{\frac{3}{4}}\left(-p_{x}+p_{y}-p_{z}\right)
\end{aligned}
$$

Show how these orbitals are obtained by following the procedure used to solve Problem 4-1.

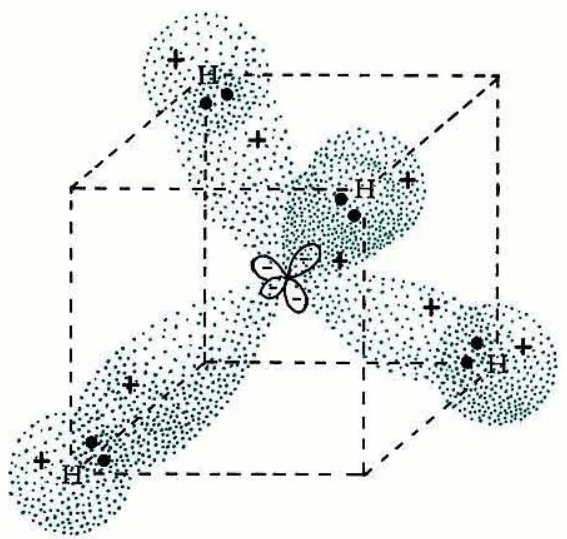

Figure 5-8 Valence-bond structure for $\mathbf{C H}_{4}$. 


\section{5-5 OTHER TETRAHEDRAL MOLECULES}

Members of the carbon family (carbon, silicon, germanium, tin, and lead) readily form four $\sigma$ bonds with four adjacent atoms. The resulting molecules invariably have a tetrahedral structure around

Table $5-1$

Properties of Tetrahedral Molecules ${ }^{a}$

\begin{tabular}{|c|c|c|c|}
\hline Molecule & Bond & $\begin{array}{c}\text { Bond } \\
\text { length, } A\end{array}$ & $\begin{array}{c}\text { Bond energy }(B E), \\
\text { kcal/mole }\end{array}$ \\
\hline $\mathrm{CH}_{4}$ & $\begin{array}{r}\mathrm{CH}_{3}-\mathrm{H} \\
\mathrm{C}-\mathrm{H}\end{array}$ & 1.093 & $\begin{array}{c}101(\mathrm{DE}) \\
99.3\end{array}$ \\
\hline $\mathrm{CF}_{4}$ & $C-F$ & 1.36 & 116 \\
\hline $\mathrm{CCl}_{4}$ & $\mathrm{C}-\mathrm{Cl}$ & 1.761 & 78.2 \\
\hline $\mathrm{CBr}_{4}$ & $\mathrm{CBr}_{3}-\mathrm{Br}$ & 1.942 & $<50(\mathrm{DE})$ \\
\hline $\mathrm{SiH}_{4}{ }^{2}$ & $\mathrm{Si}-\mathrm{H}$ & 1.480 & 76 \\
\hline $\mathrm{Si} \mathrm{F}_{4}$ & $\mathrm{Si}-\mathrm{F}$ & 1.54 & 135 \\
\hline $\mathrm{SiCl}_{4}$ & $\mathrm{Si}-\mathrm{Cl}$ & 2.02 & 91 \\
\hline $\mathrm{SiBr}_{4}$ & $\mathrm{Si}-\mathrm{Br}$ & 2.15 & 74 \\
\hline $\mathrm{SiI}_{4}$ & $\mathrm{Si}-\mathrm{I}$ & 2.43 & 56 \\
\hline $\mathrm{Si}\left(\mathrm{CH}_{3}\right)_{4}$ & $\mathrm{Si}-\mathrm{C}$ & 1.93 & 72 \\
\hline $\mathrm{Si}\left(\mathrm{C}_{2} \mathrm{H}_{5}\right)_{4}$ & $\mathrm{Si}-\mathrm{C}$ & & 60 \\
\hline $\mathrm{GeCl}_{4}$ & $\mathrm{Ge}-\mathrm{Cl}$ & 2.08 & 81 \\
\hline $\mathrm{GeBr}_{4}$ & $\mathrm{Ge}-\mathrm{Br}$ & 2.32 & 66 \\
\hline $\mathrm{GeI}_{4}$ & $\mathrm{Ge}-\mathrm{I}$ & 2.48 & 51 \\
\hline $\mathrm{SnCl}_{4}$ & $\mathrm{Sn}-\mathrm{Cl}$ & 2.30 & 76 \\
\hline $\mathrm{SnBr}_{4}$ & $\mathrm{Sn}-\mathrm{Br}$ & & 65 \\
\hline $\mathrm{Sn}\left(\mathrm{CH}_{3}\right)_{4}$ & $\mathrm{Sn}-\mathrm{C}$ & 2.18 & \\
\hline $\mathrm{Sn}\left(\mathrm{C}_{2} \mathrm{H}_{5}\right)_{4}$ & $\mathrm{Sn}-\mathrm{C}$ & & 54 \\
\hline $\mathrm{Pb}\left(\mathrm{CH}_{3}\right)_{4}$ & $\mathrm{~Pb}-\mathrm{C}$ & 2.30 & \\
\hline $\mathrm{Pb}\left(\mathrm{C}_{2} \mathrm{H}_{5}\right)_{4}$ & $\mathrm{~Pb}-\mathrm{C}$ & & 31 \\
\hline $\mathrm{SO}_{4}^{2-}$ & $\mathrm{s}-\mathrm{O}$ & 1.49 & \\
\hline $\mathrm{ClO}_{4}^{-}$ & $\mathrm{Cl}-\mathrm{O}$ & 1.44 & \\
\hline $\mathrm{NH}_{4}^{4}$ & $\mathrm{~N}-\mathrm{H}$ & 1.03 & \\
\hline $\mathrm{BH}_{4}^{-}$ & $\mathrm{B}-\mathrm{H}$ & 1.22 & \\
\hline $\mathrm{BF}_{4}^{-}$ & $B-F$ & 1.43 & \\
\hline
\end{tabular}

aData from T. L. Cottrell, The Strengths of Chemical Bonds, Butterworths, London, 1958, Table 11.5.1. 
the central atom. The bonding in these molecules involves the use of one $s$ and three $p$ valence orbitals by the central atom, and of an appropriate valence orbital by each of the four surrounding atoms.

A number of important oxyanions have a tetrahedral structure, among them $\mathrm{SO}_{4}{ }^{2-}$ and $\mathrm{ClO}_{4}{ }^{-}$. Properties of a representative group of tetrahedral molecules are given in Table 5-1.

\section{SUPPLEMENTARY PROBLEMS}

1. Describe the bonding in $\mathrm{CF}_{4}$ in terms of molecular orbitals, and construct a molecular-orbital energy-level diagram. Around which nucleus or nuclei do the electrons spend more time in the $\sigma^{b}$ orbitals? Do you expect any partial ionic character in the $\mathrm{C}-\mathrm{F}$ bonds? What is the dipole moment of $\mathrm{CF}_{4}$ ? Why?

2. Under what conditions are the molecular-orbital and valencebond descriptions of bonding in $\mathrm{CH}_{4}$ the same? From Eqs. (5-1), $(5-3),(5-5)$, and $(5-7)$, construct the valence-bond bonding functions that are shown in Fig. 5-8.

3. What is the structure of $\mathrm{BH}_{4}-$ ? of $\mathrm{NH}_{4}+$ ? Are the $\mathrm{CH}_{4}$ orbitals appropriate for these molecules? Discuss the partial ionic character you might expect in the $\mathrm{B}-\mathrm{H}, \mathrm{C}-\mathrm{H}$, and $\mathrm{N}-\mathrm{H}$ bonds. Make an estimate of the coefficients in Eqs. (5-1) through (5-8) that might be expected for the $\mathrm{BH}_{4}^{-}, \mathrm{CH}_{4}$, and $\mathrm{NH}_{4}{ }^{+}$molecules. 


\section{VI}

\section{Trigonal-Pyramidal Molecules}

\section{6-1 $\quad \mathrm{NH}_{3}$}

A familiar example of a trigonal-pyramidal molecule is ammonia, A $\mathrm{NH}_{3}$. The $\mathrm{NH}_{3}$ molecule is shown in Fig. 6-1. The three hydrogens, which are bent out of the $x, y$ plane, form the base of a trigonal pyramid that has the nitrogen at the apex. Each $\mathrm{N}-\mathrm{H}$ makes an angle $\theta$ with $z$. In addition, $\mathrm{N}-\mathrm{H}_{a}$ is lined up with the $x$ axis, and $\mathrm{N}-\mathrm{H}_{b}$ and $\mathrm{N}-\mathrm{H}_{c}$ make $30^{\circ}$ angles with $+y$ and $-y$, respectively. Thus $\mathrm{NH}_{3}$ is aligned the same way we aligned a trigonalplanar molecule (Fig. 4-1), but with the three peripheral atoms bent down.

Bonding in $\mathrm{NH}_{3}$ involves the hydrogen $1 s$ valence orbitals and the nitrogen $2 s$ and $2 p$ valence orbitals. Let us ignore the $2 s$ nitrogen orbital for the moment, and consider only the $2 p-1 s$ bonding.

The overlap of the three hydrogen $1 s$ orbitals with the nitrogen $2 p z$ orbital is shown in Fig. 6-2. The correct combination of $1 s$ orbitals is $\left(1 s_{a}+1 s_{b}+1 s_{c}\right)$. The $\sigma_{z}$ molecular orbitals are:

$$
\begin{aligned}
& \psi\left(\sigma_{z}^{b}\right)=C_{1} 2 p_{z}+C_{2}\left(1 s_{a}+1 s_{b}+1 s_{c}\right) \\
& \psi\left(\sigma_{z}^{*}\right)=C_{3} 2 p_{z}-C_{4}\left(1 s_{a}+1 s_{b}+1 s_{c}\right)
\end{aligned}
$$

The overlap of $2 p_{y}$ with $1 s_{b}$ and $1 s_{c}$ is shown in Fig. 6-3. The correct combination is $\left(1 s_{b}-1 s_{c}\right)$. The $\sigma_{y}$ molecular orbitals are: 


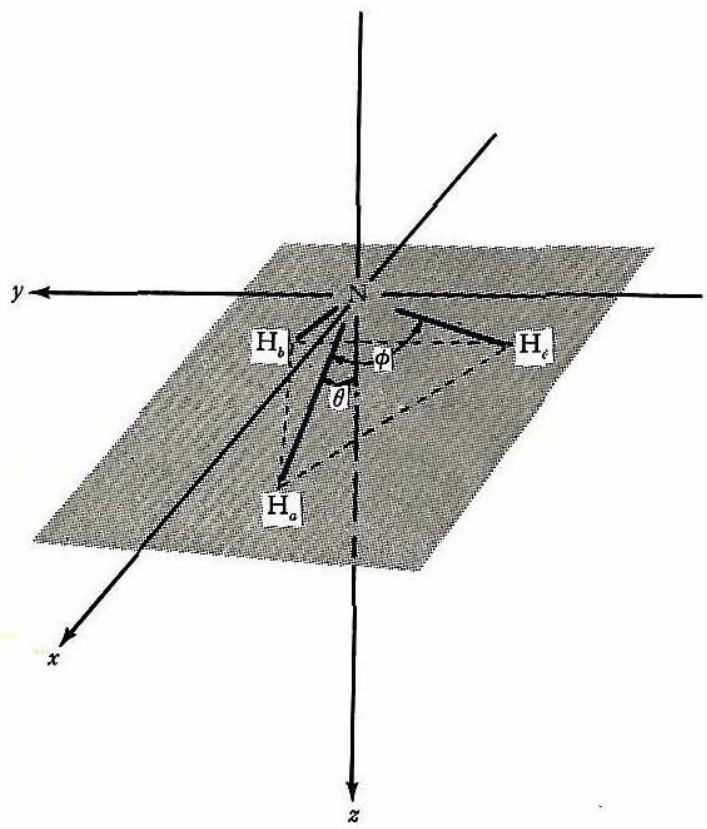

Figure 6-1 Coordinate system for $\mathbf{N H}_{3}$.

$$
\begin{aligned}
& \psi\left(\sigma_{y}{ }^{b}\right)=C_{5} 2 p_{y}+C_{6}\left(1 s_{b}-1 s_{c}\right) \\
& \psi\left(\sigma_{y}{ }^{*}\right)=C_{7} 2 p_{y}-C_{8}\left(1 s_{b}-1 s_{c}\right)
\end{aligned}
$$

The overlap of $2 p_{x}$ with $1 s_{a}, 1 s_{b}$, and $1 s_{c}$ is shown in Fig. 6-4. Since $1 s_{b}$ and $1 s_{c}$ make an angle of $60^{\circ}$ with $-x$, the overlap of $1 s_{b}$ or $1 s_{c}$ with $2 p_{x}$ is only one-half $\left(\cos 60^{\circ}=\frac{1}{2}\right)$ that of $1 s_{a}$ with $2 p_{x}$ (see Section 4-2). Thus the proper $1 s$ combination is $\left(1 s_{a}-\frac{1}{2} 1 s_{b}-\frac{1}{2} 1 s_{c}\right)$. The $\sigma_{x}$ molecular orbitals are:

$$
\begin{aligned}
& \psi\left(\sigma_{x}^{b}\right)=C_{9} 2 p_{x}+C_{10}\left(1 s_{a}-\frac{1}{2} 1 s_{b}-\frac{1}{2} 1 s_{c}\right) \\
& \psi\left(\sigma_{x}^{*}\right)=C_{11} 2 p_{x}-C_{12}\left(1 s_{a}-\frac{1}{2} 1 s_{b}-\frac{1}{2} 1 s_{c}\right)
\end{aligned}
$$

\section{6-2 OVERLAP IN $\sigma_{x}, \sigma_{y}$, AND $\sigma_{z}$}

A calculation of the overlap in the $\sigma_{x}, \sigma_{y}$, and $\sigma_{z}$ molecular orbitals is easily carried out. The direct overlap of a $2 p$ with a $1 s$ valence 


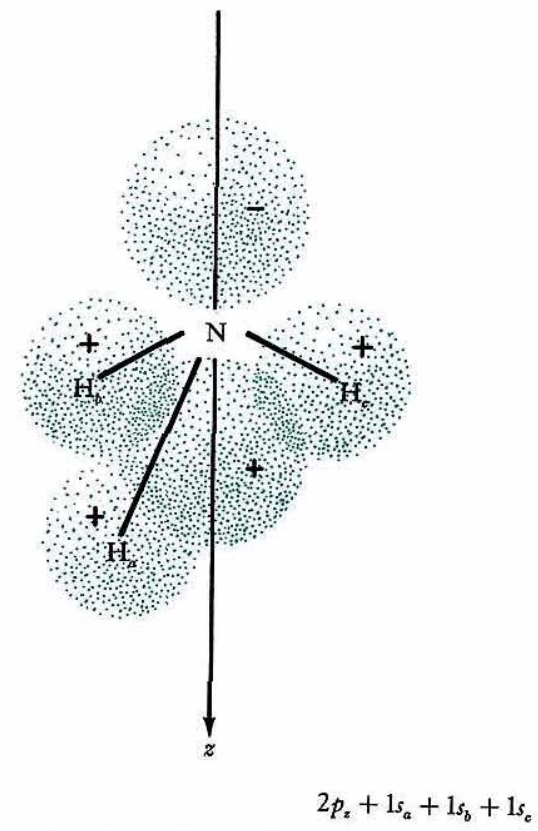

Figure 6-2 Overlap of the nitrogen $2 p_{z}$ orbital with the $1 \mathrm{~s}$ orbitals of the hydrogen atoms.

orbital is shown in Fig. 6-5; this we shall denote as $S\left(1 s, 2 p_{\sigma}\right)$. We then proceed to express the molecular-orbital overlaps in terms of $S\left(1 s, 2 p_{\sigma}\right)$ :

$$
\begin{aligned}
S\left(\sigma_{z}\right) & =\int 2 p_{z} \frac{1}{\sqrt{3}}\left(1 s_{a}+1 s_{b}+1 s_{c}\right) d \tau \\
& =\frac{\iota}{\sqrt{3}}\left[\cos \theta S\left(1 s, 2 p_{\sigma}\right)+\cos \theta S\left(1 s, 2 p_{\sigma}\right)+\cos \theta S\left(1 s, 2 p_{\sigma}\right)\right] \\
& =\sqrt{3} \cos \theta S\left(1 s, 2 p_{\sigma}\right)
\end{aligned}
$$

$$
\begin{aligned}
S\left(\sigma_{y}\right) & =\boldsymbol{S} 2 p_{y} \frac{1}{\sqrt{2}}\left(1 s_{b}-1 s_{\mathrm{c}}\right) d \tau \\
& =\frac{1}{\sqrt{2}}\left[\cos 30^{\circ} \sin \theta S\left(1 s, 2 p_{\sigma}\right)+\cos 30^{\circ} \sin \theta S\left(1 s, 2 p_{\sigma}\right)\right] \\
& =\sqrt{\frac{3}{2}} \sin \theta S\left(1 s, 2 p_{\sigma}\right)
\end{aligned}
$$




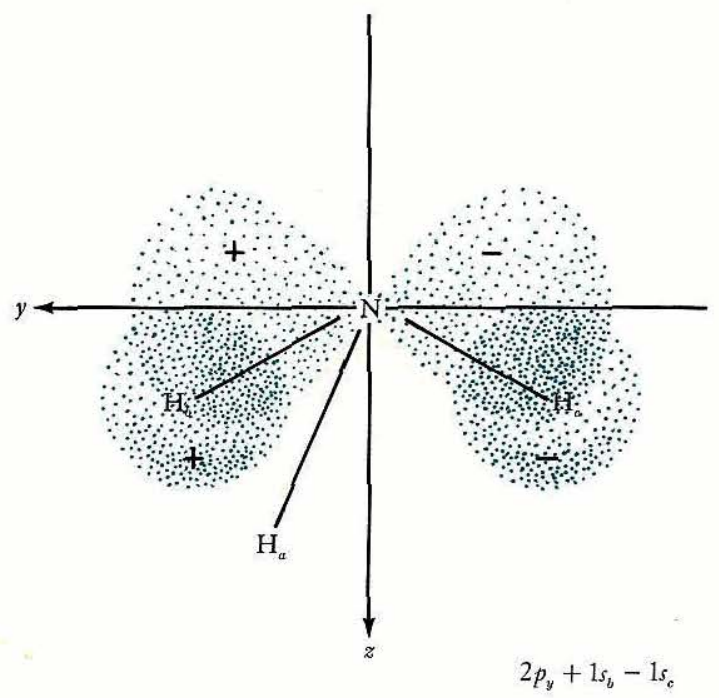

Figure 6-3 Overlap of the nitrogen $2 p_{y}$ orbital with the $1 s$ orbitals of the hydrogen atoms.

$$
\begin{aligned}
& S\left(\sigma_{x}\right)=\int 2 p_{x} \sqrt{\frac{2}{3}}\left(1 s_{a}-\frac{1}{2} 1 s_{b}-\frac{1}{2} 1 s_{c}\right) d \tau \\
&=\sqrt{\frac{2}{3}}\left[\sin \theta S\left(1 s, 2 p_{\sigma}\right)+\cos 60^{\circ} \sin \theta S\left(1 s, 2 p_{\sigma}\right)+\cos 60^{\circ} \sin \theta\right. \\
&=\sqrt{\frac{3}{2}} \sin \theta S\left(1 s, 2 p_{\sigma}\right) \\
&\left.\times S\left(1 s, 2 p_{\sigma}\right)\right]
\end{aligned}
$$

It is important to note from Eqs. (6-7), (6-8), and (6-9) that $\sigma_{y}$ and $\sigma_{x}$ are equivalent, and therefore their energies will be the same for any value of $\theta$. When $\theta=90^{\circ}$, of course, we obtain the correct overlap values for a trigonal-planar molecule (see Section 4-5):

$$
\begin{aligned}
& S\left(\sigma_{z}\right)=0 \\
& S\left(\sigma_{y}\right)=S\left(\sigma_{x}\right)=\sqrt{\frac{3}{2}} S\left(1 s, 2 p_{\sigma}\right)
\end{aligned}
$$

Let us now investigate the case for an $\mathrm{H}-\mathrm{N}-\mathrm{H}$ bond angle of $90^{\circ}$ $\left(\phi=90^{\circ}\right.$ in Fig. 6-1). Calling the $\mathrm{N}-\mathrm{H}_{a}$ length unity, the other pertinent distances given in Fig. 6-6 can be easily obtained by geom- 


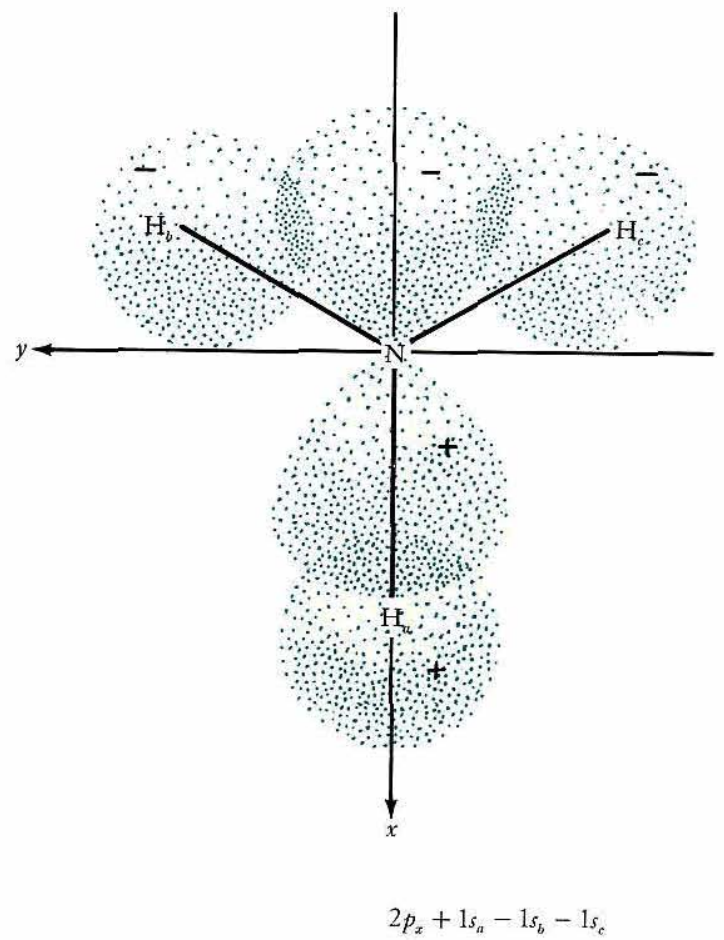

Figure 6-4 Overlap of the nitrogen $2 p_{x}$ orbital with the $1 s$ orbitals of the hydrogen atoms.

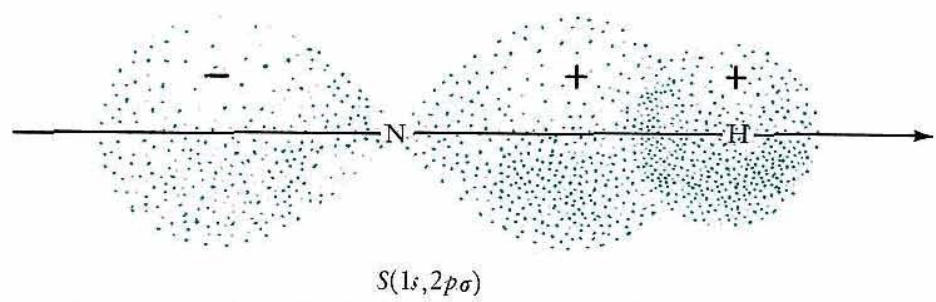

Figure 6-5 Standard two-atom $\sigma$ overlap between an $s$ and a p orbital. 


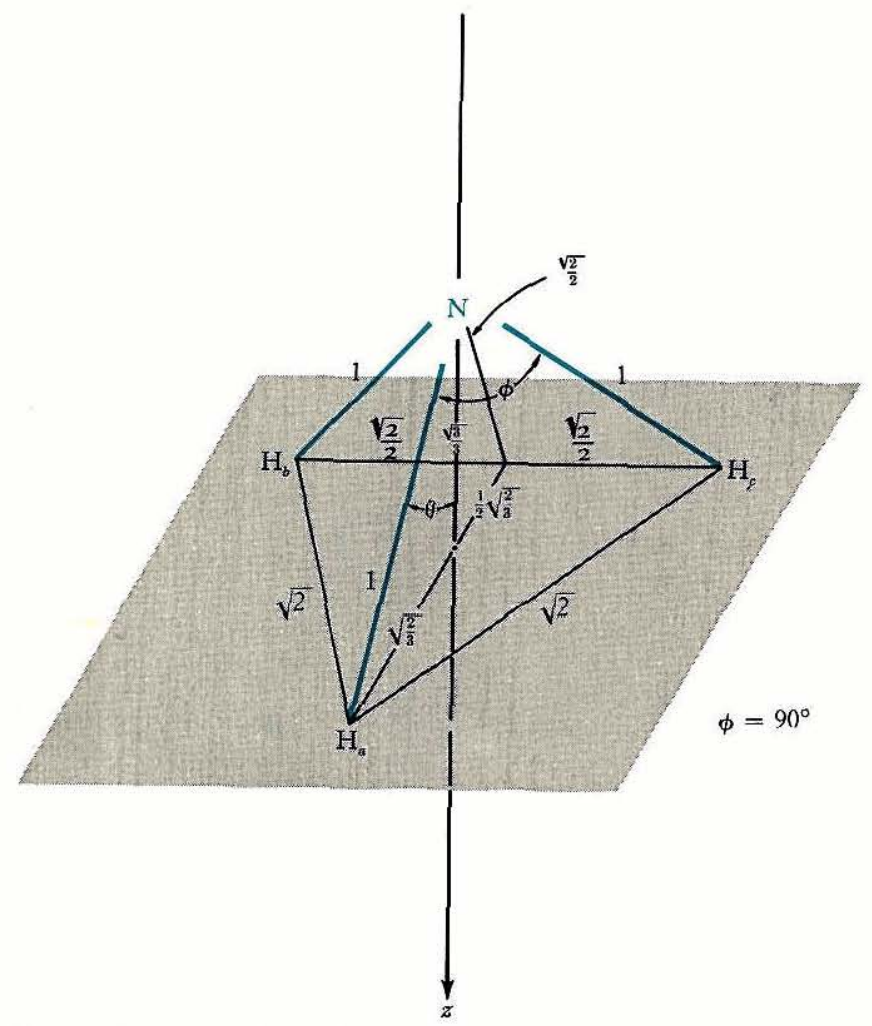

Figure 6-6 Relative distances in the $\mathrm{NH}_{3}$ molecule for an $\mathrm{H}-\mathrm{N}-\mathrm{H}$ bond angle of $90^{\circ}$.

etry. We see that for $\phi=90^{\circ}, \cos \theta=\frac{\sqrt{3}}{3}$ and $\sin \theta=\sqrt{\frac{2}{3}}$. Thus, Eqs. $(6-7),(6-8)$, and $(6-9)$ reduce to

$$
S\left(\sigma_{z}\right)=S\left(\sigma_{y}\right)=S\left(\sigma_{x}\right)=S\left(1 s, 2 p_{\sigma}\right)
$$

In other words, the $\sigma_{x}, \sigma_{y}$, and $\sigma_{z}$ molecular orbitals are the same for $\phi=90^{\circ}$. This is no surprise, since the $2 p_{x}, 2 p_{y}$, and $2 p_{z}$ orbitals make $90^{\circ}$ angles with each other, and for $\phi=90^{\circ}$ the $1 s$ orbitals can be aligned along the $x, y$, and $z$ axes, as shown in Fig. 6-7. Each hydro- 


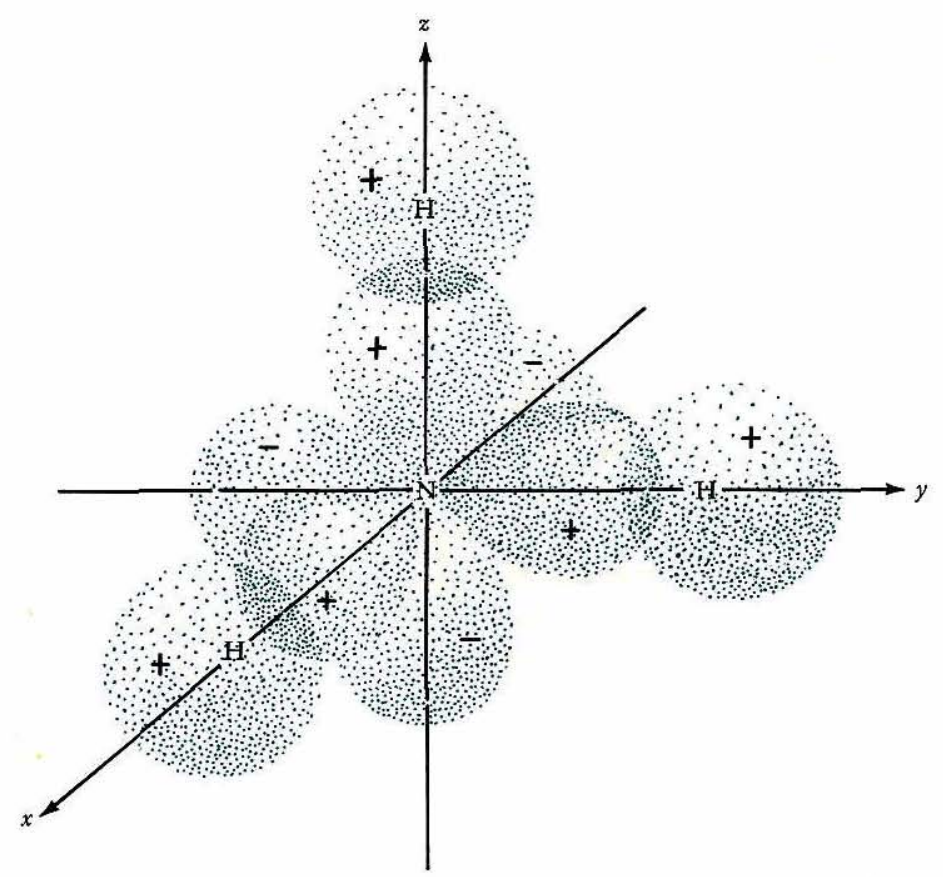

Figure 6-7 Simple picture of the bonding in $\mathbf{N H}_{3}$, using only the nitrogen $2 p$ orbitals.

gen overlaps one $2 p$ orbital, as in Eq. (6-10). The total overlap in $\sigma_{x}, \sigma_{y}$, and $\sigma_{z}$ is smaller for any other angle.

\section{6-3 THE INTERELECTRONIC REPULSIONS AND $\mathrm{H}-\mathrm{N}-\mathrm{H}$ BOND ANGLE IN $\mathrm{NH}_{3}$}

The actual $\mathrm{H}-\mathrm{N}-\mathrm{H}$ bond angle in $\mathrm{NH}_{3}$ is $107^{\circ}$, or $17^{\circ}$ larger than the angle predicted for pure $2 p-1 s$ bonding. It is probable that the mutual repulsions of the one nonbonding pair (called a lone pair) and the three bonding pairs of electrons are responsible for the $17^{\circ}$ angle opening. The four electron pairs must therefore be so arranged as to minimize these interelectronic repulsions. One way to get the three 
bonding pairs farther apart is to involve the nitrogen $2 s$ orbital in the bonding. In Fig. 6-8 is shown the overlap of the hydrogen $1 s$ orbitals with the nitrogen $2 s$. Notice that the combination appropriate for $2 s\left(1 s_{a}+1 s_{b}+1 s_{c}\right)$ is the $1 s$ combination in $\sigma_{z}$ [Eqs. (6-1) and $(6-2)]$. Thus $\sigma_{s}$ " mixes together" with $\sigma_{z}{ }^{b}$ and $\sigma_{z}{ }^{*}$ to give three molecular orbitals which we shall call $\sigma_{s}^{b}, \sigma_{z}$, and $\sigma_{z}{ }^{*}$. This addition of $2 s$ "character" to the $\mathrm{N}-\mathrm{H}$ bonding increases the $\mathrm{H}-\mathrm{N}-\mathrm{H}$ angle from $90^{\circ}$ to $107^{\circ}$. You may think of the angle opening by inclusion of $2 s$ in the following way: The best $\mathrm{H}-\mathrm{N}-\mathrm{H}$ angle for "pure" $2 p$ bonding is $90^{\circ}$. The best $\mathrm{H}-\mathrm{N}-\mathrm{H}$ angle for "pure" $2 s$ bonding is $120^{\circ}$, since the symmetrical trigonal planar structure allows the best overlap arrangement for three hydrogen $1 s$ orbitals with a $2 s$ orbital. (The $1 s$ orbitals are as far from each other as possible and do not compete for overlap of the same portion of the $2 s$.) Thus inclusion of $2 s$ character in a "pure" $2 p$-bonding scheme increases the $\mathrm{H}-\mathrm{N}-\mathrm{H}$ angle from $90^{\circ}$.

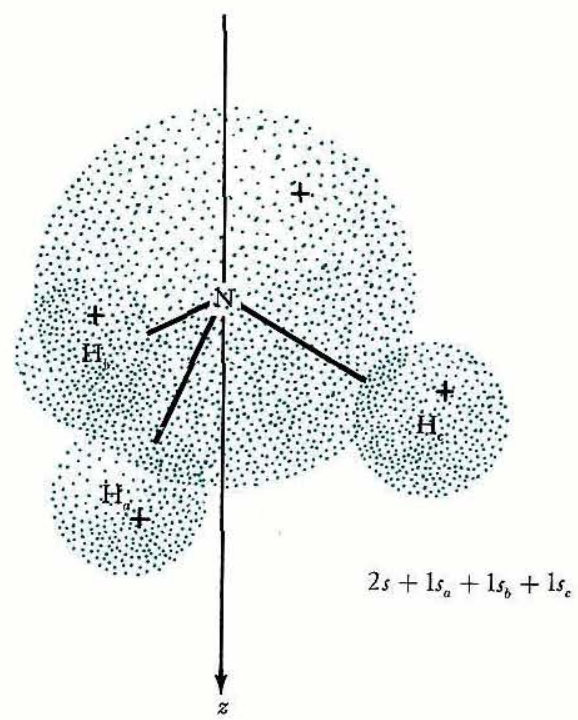

Figure 6-8 Overlap of the nitrogen $2 s$ orbital with the $1 s$ orbitals of the hydrogen atoms. 
The similar valence-bond idea, particularly appealing, is that the bonding pairs and the lone pair are in four tetrahedral $s p^{3}$ orbitals. This structure places the four electron pairs as far away from each other as possible. The "tetrahedral" structure of $\mathrm{NH}_{3}$ is shown in Fig. 6-9. The slight deviation of the $\mathrm{H}-\mathrm{N}-\mathrm{H}$ bond angle from the tetrahedral angle of $109^{\circ}$ is considered a result of the nonequivalence of the bonding and non-bonding pairs of electrons.

\section{6-4 BOND ANGLES OF OTHER TRIGONAL-PYRAMIDAL MOLECULES}

The $\mathrm{H}-\mathrm{P}-\mathrm{H}$ and $\mathrm{H}-\mathrm{As}-\mathrm{H}$ angles in $\mathrm{PH}_{3}$ and $\mathrm{AsH}_{3}$ are $94^{\circ}$ and $92^{\circ}$, respectively. This probably indicates a high degree of phosphorus and arsenic $p$-orbital character in the three bonding orbitals. We assume that the mutual repulsions of bonding pairs of electrons are reduced in going from nitrogen to phosphorus to arsenic. This is a reasonable assumption, since we know from atomic spectra that the atomic interelectronic repulsions, in the valence $p$ orbitals, decrease in the order $N>P>$ As. The trihalides of nitrogen, phosphorus, arsenic, antimony, and bismuth are trigonal pyramidal. The bond angles are all in the 95 to $105^{\circ}$ range, as given in Table 6-1.

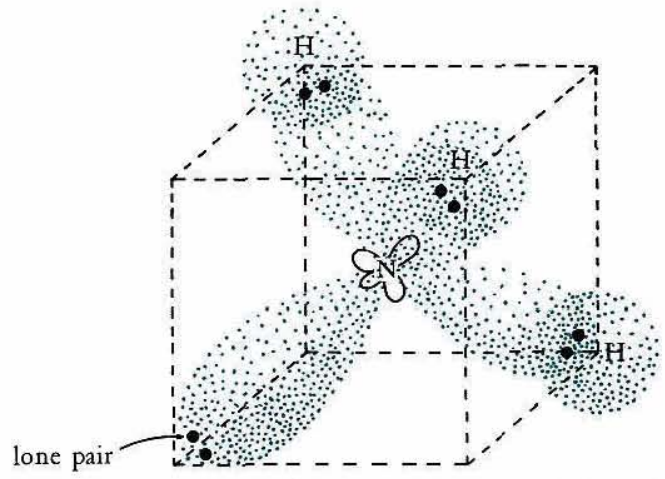

Figure 6-9 Valence-bond structure for $\mathrm{NH}_{3}$, using $\boldsymbol{s p}^{3}$ orbitals for nitrogen. 
Tab 6 - $6=1$

Propertier of Trigonal- Pyramidal Molecules"

\begin{tabular}{lclc}
$\begin{array}{c}A_{3} B_{3} \\
\text { molecule }\end{array}$ & $\begin{array}{c}B-A-B \\
\text { angle, deg }\end{array}$ & $\begin{array}{c}\text { AB bond } \\
\text { length, } A\end{array}$ & $\begin{array}{c}\text { AB bond energy }(B E), \\
\text { kcal/mole }\end{array}$ \\
\hline $\mathrm{NH}_{3}$ & 107 & 1.014 & 93.4 \\
$\mathrm{NF}_{3}$ & 103 & 1.37 & 65 \\
$\mathrm{NCl}_{3}$ & & & 46 \\
$\mathrm{PH}_{3}$ & 94 & 1.42 & 77 \\
$\mathrm{PF}_{3}$ & 104 & & 117 \\
$\mathrm{PCl}_{3}$ & 100 & 2.04 & 78 \\
$\mathrm{PBr}_{3}$ & 100 & 2.20 & 63 \\
$\mathrm{PI}_{3}$ & & 2.47 & 44 \\
$\mathrm{AsH}_{3}$ & 92 & 1.52 & 59 \\
$\mathrm{AsF}_{3}$ & 102 & 1.71 & 111 \\
$\mathrm{AsCl}$ & 98 & 2.16 & 70 \\
$\mathrm{AsBr}$ & & 2.33 & 58 \\
$\mathrm{AsI}$ & & 2.54 & 43 \\
$\mathrm{SbCl}_{3}$ & 104 & 2.48 & 67 \\
$\mathrm{BiCl}_{3}$ & & 2.48 & 67 \\
$\mathrm{Bi}\left(\mathrm{CH}_{3}\right)_{3}$ & & 2.30 & 31 \\
\hline
\end{tabular}

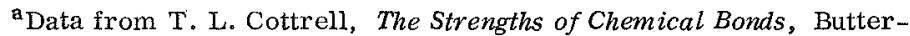
worths, London, 1958, Table 11.5.1; L. E. Sutton (ed.), "Interatomic Distances," Special Publication No. 11, The Chemical Society, London, 1958.

\section{6-5 Ground state of $\mathrm{NH}_{3}$}

The molecular-orbital energy-level scheme for $\mathrm{NH}_{3}$ is shown in Fig. 6-10. The $\sigma_{x}$ and $\sigma_{y}$ orbitals are degenerate. The eight valence electrons give a ground-state configuration of

$$
\left(\sigma_{s}^{b}\right)^{2}\left(\sigma_{x}^{b}\right)^{2}\left(\sigma_{y}^{b}\right)^{2}\left(\sigma_{z}\right)^{2} \quad S=0
$$

There are three $\sigma$ bonds. The $\mathrm{N}-\mathrm{H}$ bond length is $1.014 \mathrm{~A}$, and the average $\mathrm{N}-\mathrm{H}$ bond energy is $93.4 \mathrm{kcal} /$ mole. The electrons in the bonding orbitals spend more time around nitrogen than around the hydrogens. This means that in the ground state the nitrogen has a small negative charge and the hydrogens carry a small positive charge. Thus there are three bond dipoles, as shown in Fig. 6-11. These three bond dipoles add vectorially to give $\mathrm{NH}_{3}$ a net dipole 


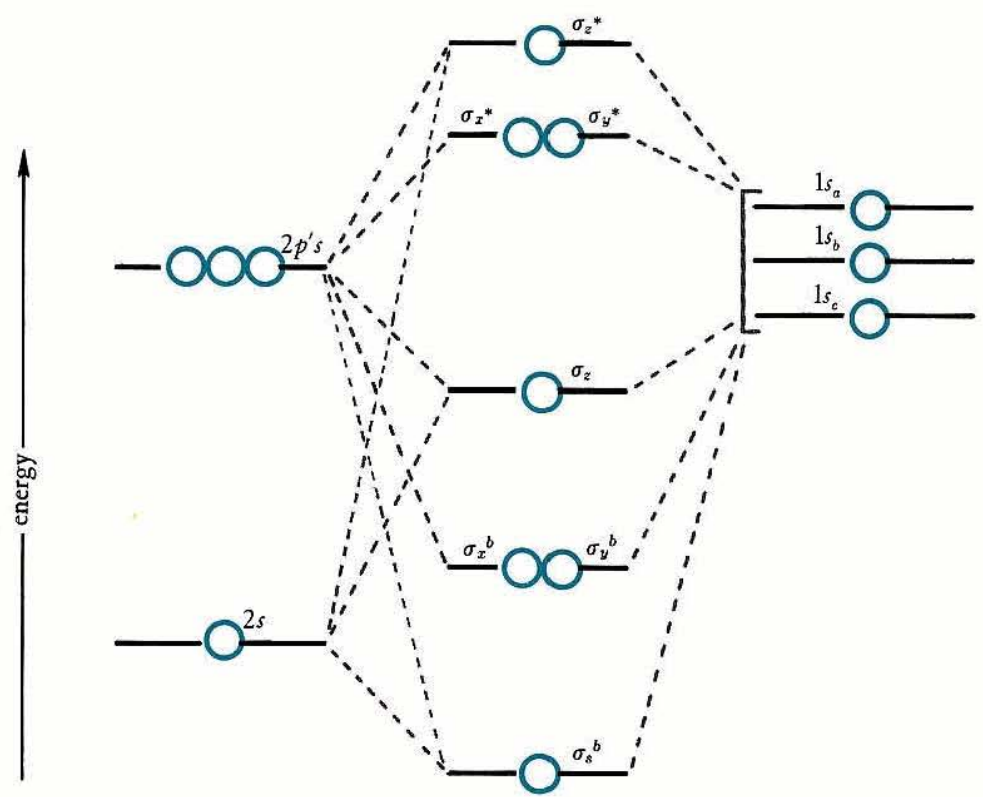

Figure 6-10 Relative orbital energies in $\mathbf{N H}_{3}$.
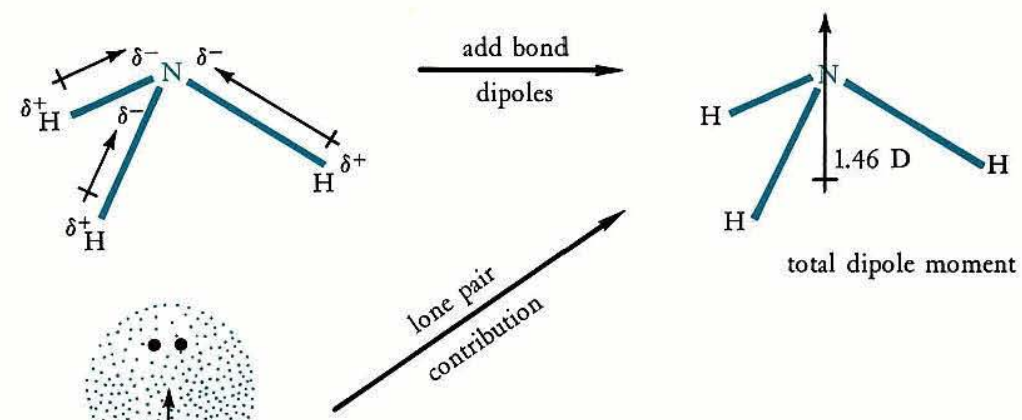

total dipole moment

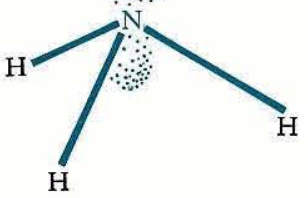

Figure 6-11 Contributions to the dipole moment of $\mathrm{NH}_{3}$. 
Table $6-2$

Dipole Moments of Some Trigonal-Pyramidal Molecules ${ }^{a}$

\begin{tabular}{ll}
\hline Molecule & Dipole moment, $D$ \\
\hline $\mathrm{NH}_{3}$ & 1.47 \\
$\mathrm{NF}_{3}$ & 0.23 \\
$\mathrm{PH}_{3}$ & 0.55 \\
$\mathrm{PF}_{3}$ & 1.03 \\
$\mathrm{PCl}_{3}$ & 0.79 \\
$\mathrm{PBr}_{3}$ & 0.61 \\
$\mathrm{AsH}_{3}$ & 0.15 \\
$\mathrm{AsF}_{3}$ & 2.82 \\
$\mathrm{AsCl}_{3}$ & 1.99 \\
$\mathrm{AsBr}_{3}$ & 1.67 \\
$\mathrm{AsI}_{3}$ & 0.97 \\
$\mathrm{SbCl}_{3}$ & 3.93 \\
$\mathrm{SbBr}_{3}$ & 2.48 \\
$\mathrm{SbI}_{3}$ & 1.59 \\
\hline
\end{tabular}

${ }^{\text {a}}$ Data from A. L. McClellan, Tables of Experimental Dipole Moments, Freeman, San Francisco, 1963:

moment. The total dipole moment, $1.46 \mathrm{D}$, also includes a contribution from the lone-pair electrons in $\sigma_{z}$, as indicated in Fig. 6-11.

Dipole moments for a number of trigonal-pyramidal molecules are given in Table 6-2.

\section{SUPPLEMENTARY PROBLEMS}

l. Why is the dipole moment of $\mathrm{NH}_{3}$ larger than the dipole moment of $\mathrm{PH}_{3}$ ? Why is the dipole moment of $\mathrm{PF}_{3}$ larger than that of $\mathrm{PCl}_{3}$ ?

2. What structure would you expect for $\mathrm{CH}_{3}^{-}$and $\mathrm{H}_{3} \mathrm{O}^{+}$. Discuss the bonding in these molecules. 


\section{VII}

\section{Angular Triatomic Molecules}

\section{7-1 $\quad \mathrm{H}_{2} \mathrm{O}$}

The most familiat angular triatomic molecule is water, $\mathrm{H}_{2} \mathrm{O}$. The $\mathrm{H}-\mathrm{O}-\mathrm{H}$ bond angle in the water molecule is known to be $105^{\circ}$. We can conveniently derive the molecular orbitals for $\mathrm{H}_{2} \mathrm{O}$ by placing the oxygen atom at the origin of an $x y z$ coordinate system. The two hydrogens are placed in the $x, z$ plane, as shown in Fig. 7-1. Imagine starting with a linear $\mathrm{H}-\mathrm{O}-\mathrm{H}$ along the $z$ axis and bending the two hydrogens toward the $x$ axis, until the $\mathrm{H}-\mathrm{O}-\mathrm{H}$ angle $\theta$ corresponds to the observed $105^{\circ}$. It is convenient to bend each hydrogen the same amount from the $z$ axis, so that the $x$ axis bisects $\theta$. We can go through this procedure for any angular triatomic molecule, independent of the value of $\theta$. Thus the $\sigma$ molecular orbitals for $\mathrm{H}_{2} \mathrm{O}$ are a representative set.

The valence orbitals involved are $2 s$ and $2 p$ for oxygen and $1 s$ for hydrogen. The overlaps of the $2 p$ orbitals with the two hydrogen 1s orbitals are shown in Fig. 7-2. From these overlaps, we can write the following set of wave functions:

$$
\begin{aligned}
& \psi\left(\sigma_{x}^{b}\right)=C_{1} 2 p_{x}+C_{2}\left(1 s_{a}+1 s_{b}\right) \\
& \psi\left(\sigma_{x}^{*}\right)=C_{3} 2 p_{x}-C_{4}\left(1 s_{a}+1 s_{b}\right) \\
& \psi\left(\sigma_{z}^{b}\right)=C_{5} 2 p_{z}+C_{6}\left(1 s_{a}-1 s_{b}\right) \\
& \psi\left(\sigma_{z}^{*}\right)=C_{7} 2 p_{z}-C_{8}\left(1 s_{a}-1 s_{b}\right)
\end{aligned}
$$




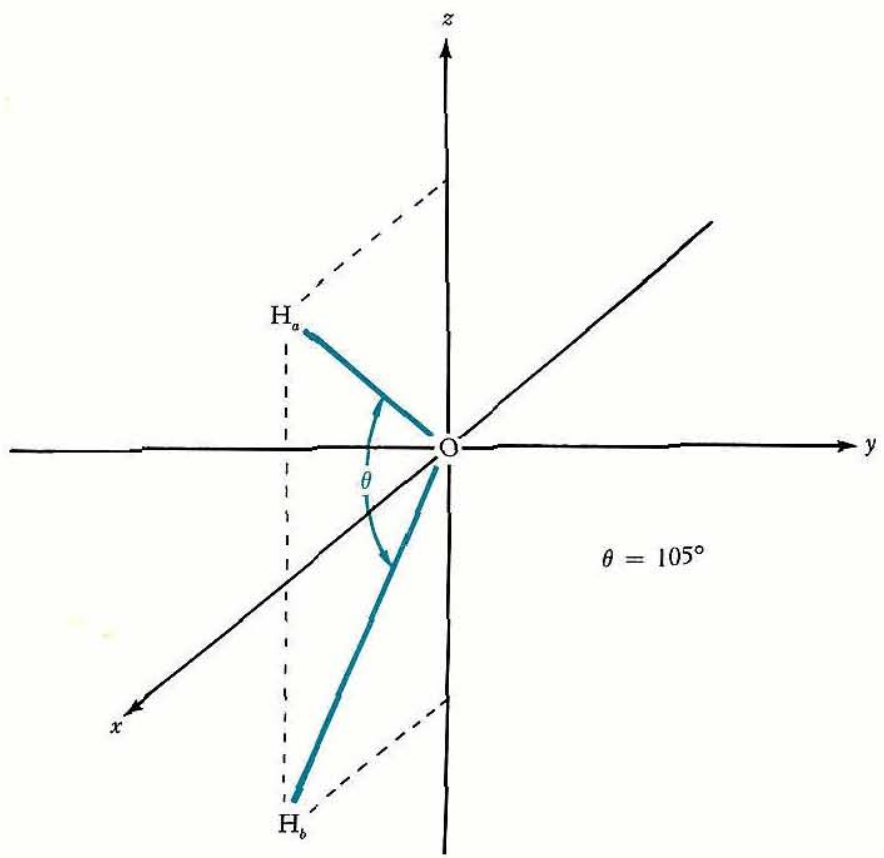

Figure 7-1 Coordinate system for $\mathrm{H}_{2} \mathrm{O}$.

The $2 p_{y}$ oxygen orbital has no overlap with either $1 s_{a}$ or $1 s_{b}$, and thus it is nonbonding in our scheme. Notice that $2 p_{y}$ is available for $\pi$ bonding, but hydrogens do not have $\pi$ valence orbitals.

The overlap of $2 s$ with $1 s_{a}$ and $1 s_{b}$ is shown in Fig. 7-3. The combination $\left(1 s_{a}+1 s_{b}\right)$, which was used in the $\sigma_{x}$ orbitals, is correct for $2 s$. This means that $\sigma_{s}$ mixes with $\sigma_{x}$. The result is three molecular orbitals-a bonding orbital, an orbital that is nearly nonbonding, and an antibonding orbital. We shall call these orbitals $\sigma_{s}^{b}, \sigma_{x}$, and $\sigma_{x}{ }^{*}$, respectively.

The molecular-orbital energy-level scheme is shown in Fig. 7-4, with the hydrogen $1 s$ orbital placed above the oxygen $2 s$ and $2 p$ valence orbitals. The $\sigma_{z}{ }^{b}$ molecular orbital is seen to be more stable than the $\sigma_{x}$, owing to the interaction of $\sigma_{x}$ with $\sigma_{s}^{b}$. 

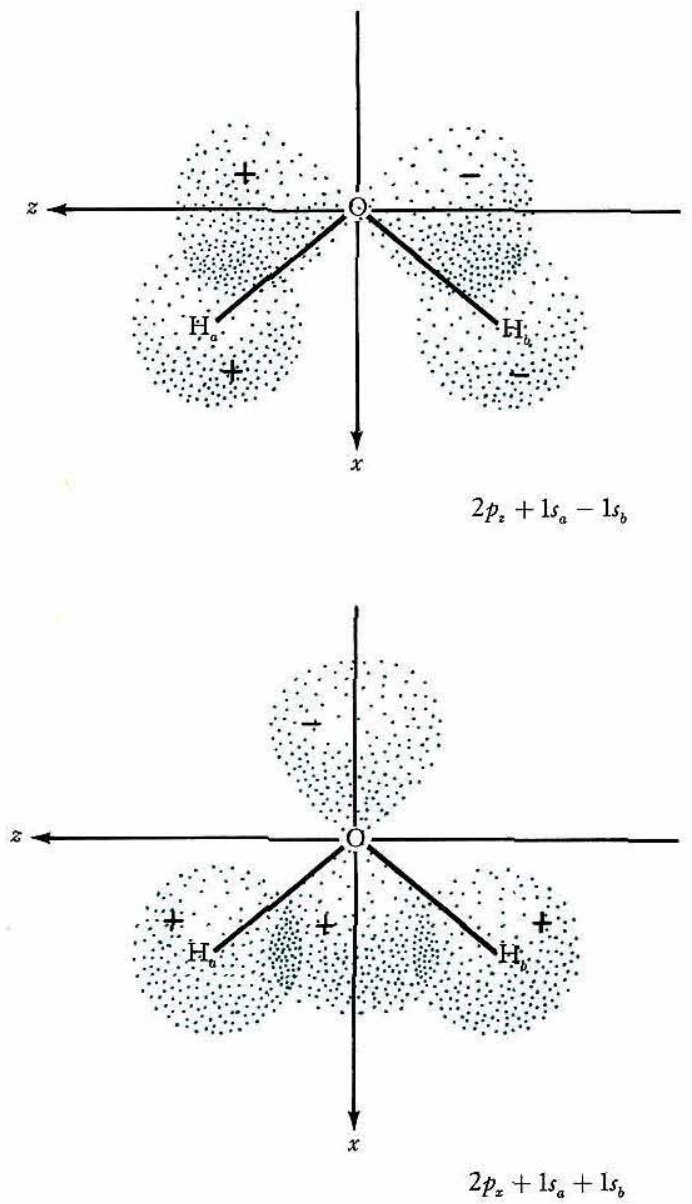

Figure 7-2 Overlap of the oxygen $2 p_{z}$ and $2 p_{x}$ orbitals with the $1 s$ orbitals of the hydrogen atoms.

\section{7-2 GROUND STATE OF $\mathrm{H}_{2} \mathrm{O}$}

The ground-state electronic configuration of $\mathrm{H}_{2} \mathrm{O}$, with eight valence electrons (two from the hydrogens, $2 s^{2} 2 p^{4}$ or six from oxygen), is therefore 


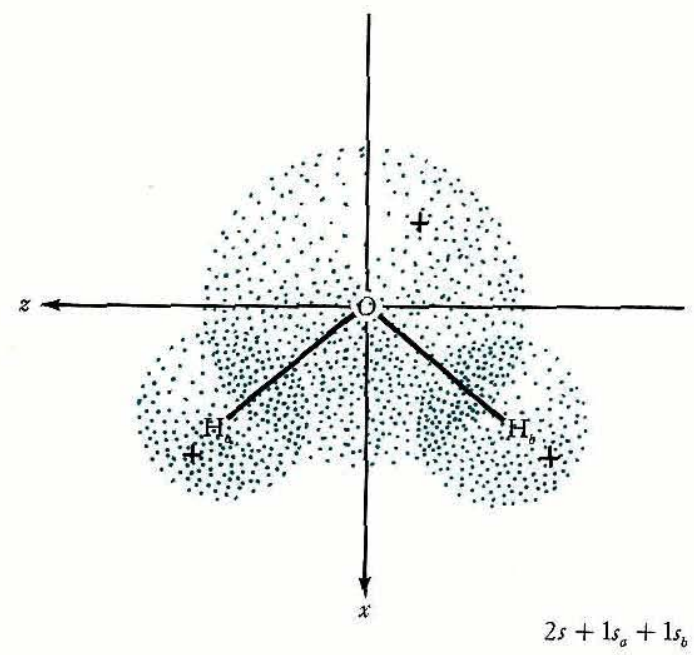

Figure 7-3 Overlap of the oxygen $2 s$ orbital with the $1 s$ orbitals of the hydrogen atoms.

$$
\left(\sigma_{s}^{b}\right)^{2}\left(\sigma_{z}^{b}\right)^{2}\left(\sigma_{x}\right)^{2}\left(\pi_{y}\right)^{2} \quad S=0
$$

We note that all the electrons must be paired, and $\mathrm{H}_{2} \mathrm{O}$ is diamagnetic. There are four electrons in $\sigma^{b}$ orbitals, giving two $\sigma$ bonds.

We might expect the $\mathrm{H}-\mathrm{O}-\mathrm{H}$ bond angle to be $90^{\circ}$ if only the $2 p_{x}$ and $2 p_{z}$ orbitals were used in $\sigma$ bonding. That is, a $\theta$ of $90^{\circ}$ makes $2 p_{x}$ and $2 p_{z}$ equivalent with respect to overlap with the $H$ valence orbitals. This is easy to see if we place the two hydrogens along the $x$ and $z$ axes, as shown in Fig. 7-5. The possibility of the $2 s$ orbital being involved in bonding is one explanation for the $15^{\circ}$ deviation of the $\mathrm{H}-\mathrm{O}-\mathrm{H}$ angle from $90^{\circ}$. To demonstrate the angle "opening," it is convenient (as for $\mathrm{NH}_{3}$ ) to place the eight valence electrons into four $s p^{3}$ hybrid orbitals, as shown in Fig. 7-6. The fact that the $\mathrm{H}-\mathrm{O}-\mathrm{H}$ angle in water is less than $109^{\circ}$ is, according to this view, a result of the different repulsions of electron pairs in bonding and nonbonding orbitals. The nonbonding pairs would repel each other more strongly than the bonding pairs, consistent with a $105^{\circ}$ angle between the bonding pairs. 
O orbitals

$\mathrm{H}_{2} \mathrm{O}$ orbitals

$\mathrm{H}$ orbitals

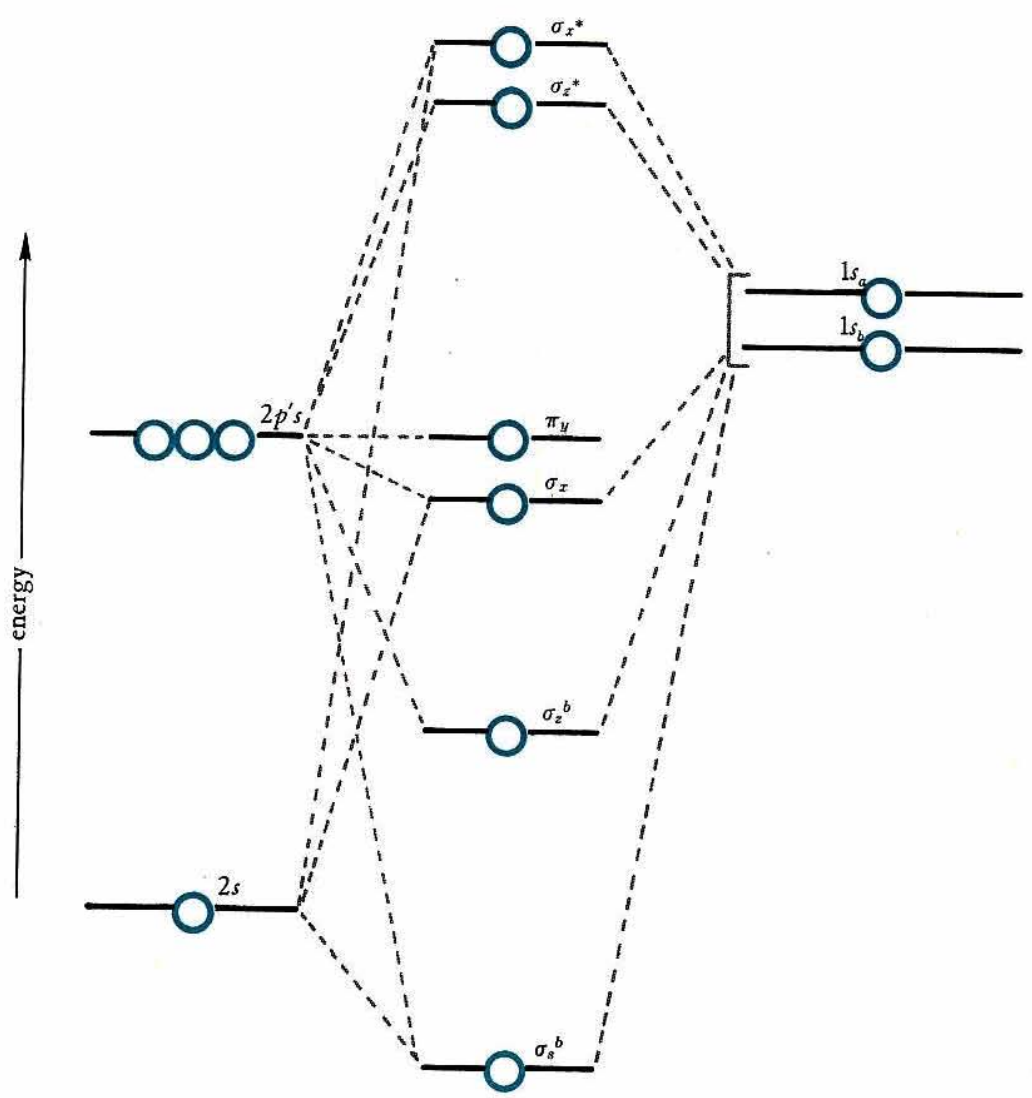

Figure 7-4 Relative orbital energies in $\mathrm{H}_{2} \mathrm{O}$.

The bond angle in $\mathrm{H}_{2} \mathrm{~S}$ is $92^{\circ}$, much closer to the $90^{\circ}$ expected for pure $p$ bonding. In $\mathrm{H}_{2} \mathrm{~S}$, it is probable that there is strong $3 p-1$ s bonding. This is consistent with the fact that the interelectronic repulsions in $3 p$ orbitals on sulfur are known to be less than the interelectronic repulsions in $2 p$ orbitals on oxygen.

The electrons in $\sigma^{b}$ orbitals in $\mathrm{H}_{2} \mathrm{O}$ spend more time near the oxygen than near the hydrogens, owing to the larger electronegativity of 


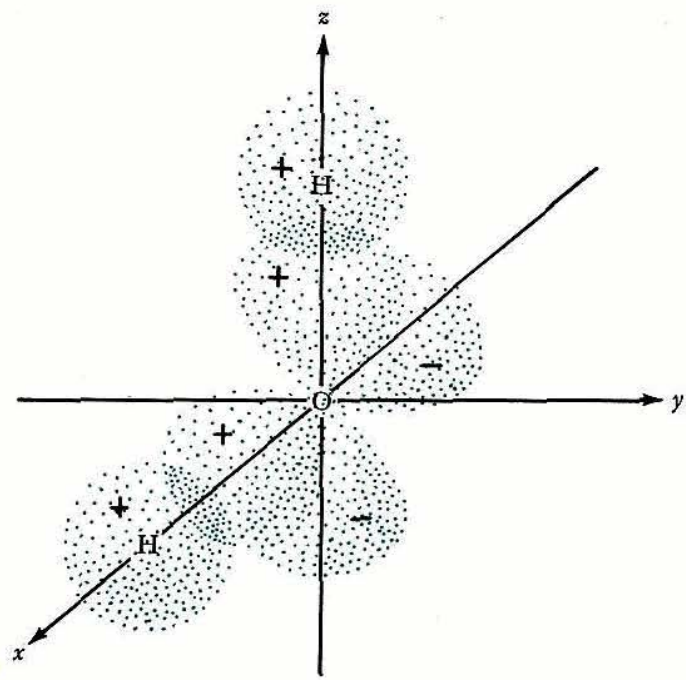

Figure 7-5 Simple picture of the bonding in $\mathrm{H}_{2} \mathrm{O}$, using only the oxygen $2 p$ orbitals.

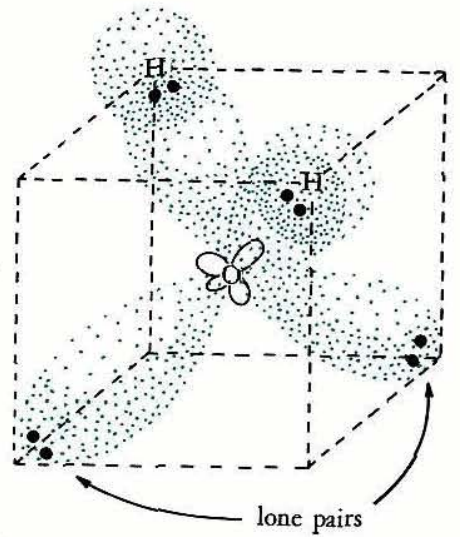

Figure 7-6 Valence-bond structure for $\mathrm{H}_{2} \mathrm{O}$, using $s p^{3}$ orbitals for oxygen. 


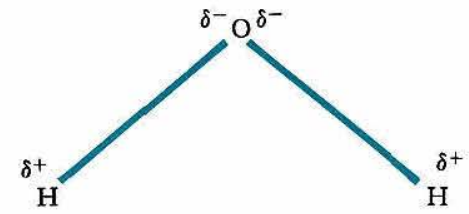

Figure 7-7 Separation of charge in $\mathrm{H}_{2} \mathrm{O}$ in the ground state.

oxygen. As a result, the hydrogens carry a small positive charge in the ground state of $\mathrm{H}_{2} \mathrm{O}$, as shown in Fig. 7-7.

The $\mathrm{H}_{2} \mathrm{O}$ molecule has a dipole moment of $1.844 \mathrm{D}$. The moment is due to the charge separation described above as well as to lone pairs, as shuwn in Fig. 7-8. Each $\mathrm{H}-\mathrm{O}$ bond has a small bond dipole moment resulting from the charge separation $\stackrel{\delta+}{\mathrm{H}}-\stackrel{\delta-}{\mathrm{O}}$. Since the $\mathrm{H}_{2} \mathrm{O}$ molecule is angular, these bond moments add together to give a resultant dipole moment.

Table 7-1 gives dipole moments of several angular triatomic molecules.

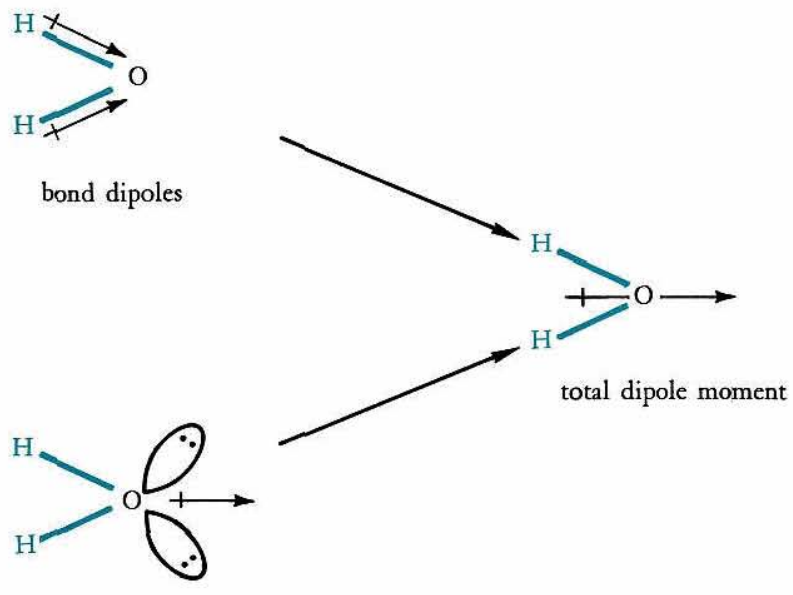

lone pairs

Figure 7-8 Contributions to the dipole moment of $\mathrm{H}_{2} \mathrm{O}$. 
Table $7-1$

Dipole Moments of Some Angular Triatomic Molecules ${ }^{a}$

\begin{tabular}{cc}
\hline Molecule & Dipole moment, $D$ \\
\hline $\mathrm{H}_{2} \mathrm{O}$ & 1.844 \\
$\mathrm{H}_{2} \mathrm{~S}$ & 0.92 \\
$\mathrm{SO}_{2}$ & 1.633 \\
$\mathrm{NO}_{2}$ & 0.39 \\
$\mathrm{O}_{3}$ & 0.52 \\
\hline
\end{tabular}

${ }^{a}$ Data from A. L. McClellan, Tables of Experimental Dipole Moments, Freeman, San Francisco, 1963.

7-3 ANGULAR TRIATOMIC MOLECULES WITH $\pi$ BONDING: $\mathrm{NO}_{2}$

The $\mathrm{NO}_{2}$ molecule is an example of an angular triatomic molecule with both $\sigma$ and $\pi$ bonding. We place the $\mathrm{N}$ of $\mathrm{NO}_{2}$ at the origin of an $x y z$ coordinate system shown in Fig. 7-9. The oxygens are situated in the $x z$ plane, bent away from the $z$ axis. The $\mathrm{O}-\mathrm{N}-\mathrm{O}$ angle is $\theta$. We shall consider the nitrogen $2 s$ and $2 p$ and the oxygen $2 p$ orbitals in constructing the molecular orbitals.

\section{7-4 $\sigma$ ORBITALS}

The nitrogen $2 s, 2 p_{x}$, and $2 p_{z}$ valence orbitals are used to form $\sigma$ molecular orbitals with the $2 p_{z_{a}}$ and $2 p_{z_{b}}$ of the oxygens. The $\sigma$ molecular orbitals are very similar to those we obtained for $\mathrm{H}_{2} \mathrm{O}$. In order of increasing energy, we have $\sigma_{s}^{b}, \sigma_{z}^{b}, \sigma_{x}, \sigma_{z}^{*}$, and $\sigma_{x}{ }^{*}$ (see Fig. 7-4).

\section{$7-5 \pi$ ORBITALS}

The nitrogen $2 p_{y}$ orbital overlaps the $2 p_{y_{a}}$ and $2 p_{y_{b}}$ on the oxygens, as shown in Fig. 7-10. The bonding molecular orbital is obtained by adding the three orbitals together:

$$
\psi\left(\pi_{y}^{b}\right)=C_{1} 2 p_{y}+C_{2}\left(y_{a}+y_{b}\right)
$$




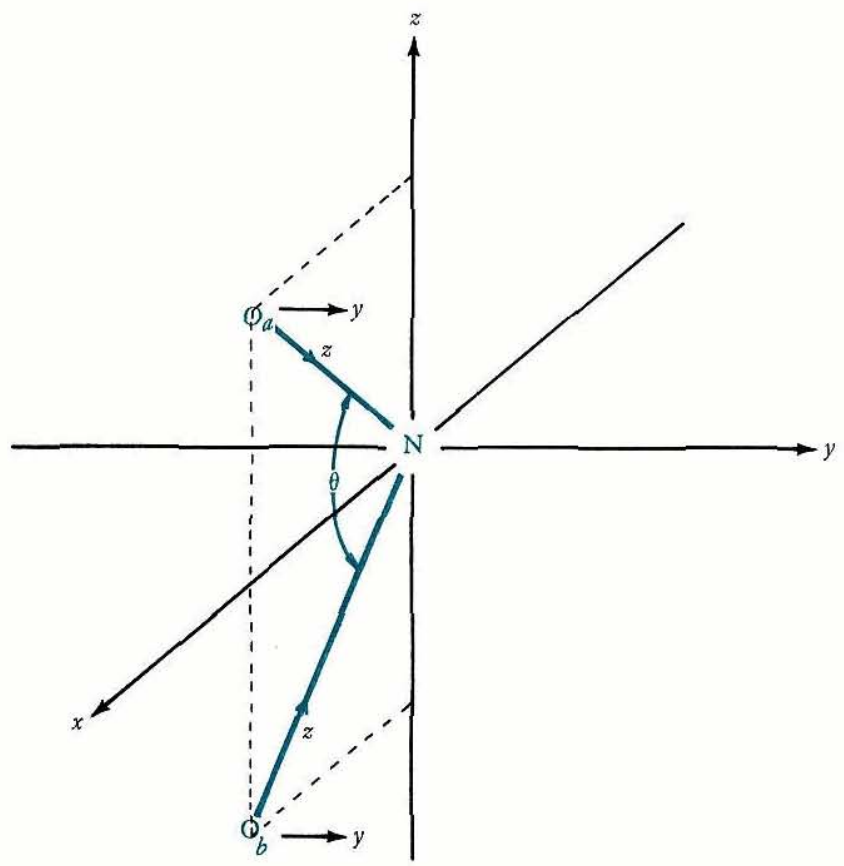

Figure 7-9 Coordinate system for $\mathrm{NO}_{2}$.

The antibonding orbital has a node between $\mathrm{O}_{a}$ and $\mathrm{N}$ and between $\mathrm{O}_{b}$ and $\mathrm{N}$ :

$$
\psi\left(\pi_{y}^{*}\right)=C_{3} 2 p_{y}-C_{4}\left(y_{a}+y_{b}\right)
$$

The other combination of the $2 p_{y}$ orbitals of $\mathrm{O}_{a}$ and $\mathrm{O}_{b}$ is $\left(2 p_{y_{a}}-2 p_{y_{b}}\right)$. This combination has zero net overlap with the nitrogen $2 p_{y}$, and is the nonbonding molecular orbital:

$$
\psi\left(\pi_{y}\right)=\frac{1}{\sqrt{2}}\left(y_{a}-y_{b}\right)
$$

We shall also consider the $2 p_{x}$ orbitals of $\mathrm{O}_{a}$ and $\mathrm{O}_{b}$ nonbonding in $\mathrm{NO}_{2}$. An approximate energy-level scheme for the molecular orbitals of $\mathrm{NO}_{2}$ is given in Fig. 7-11. 


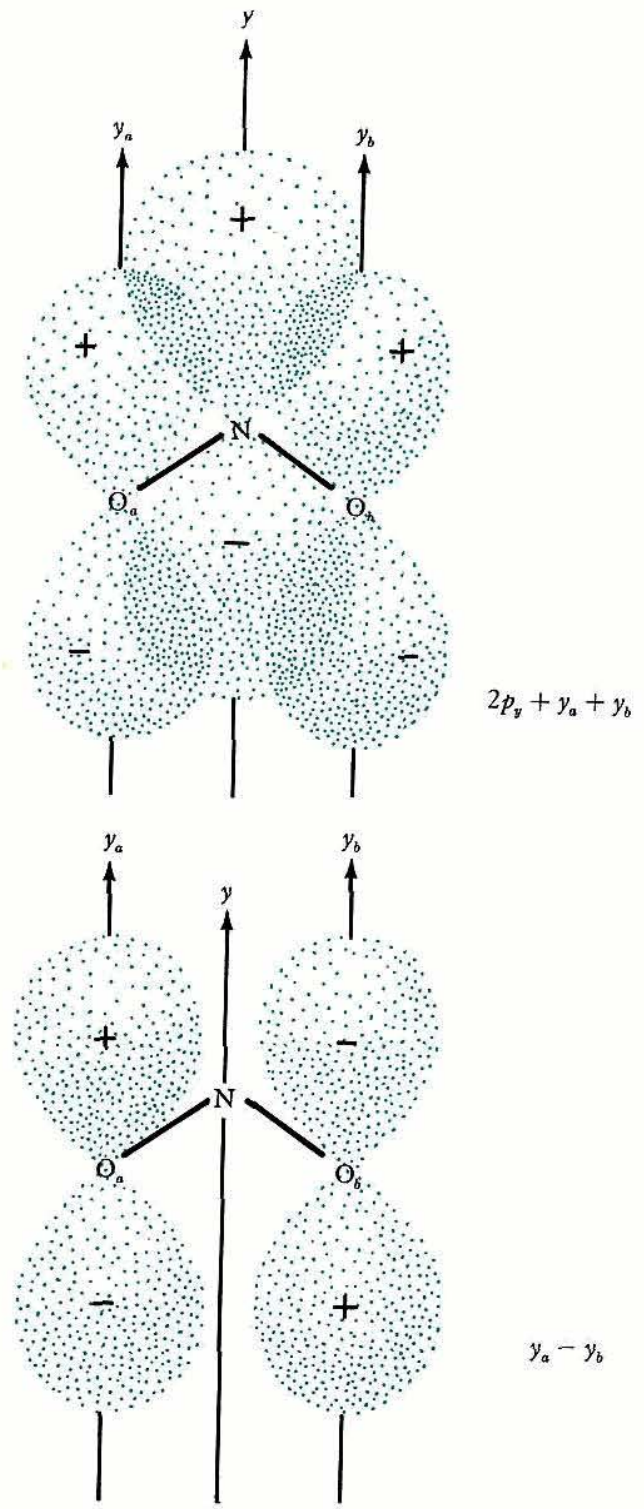

Figure 7-10 The $\pi$-orbital combinations in $\mathrm{NO}_{2}$. 


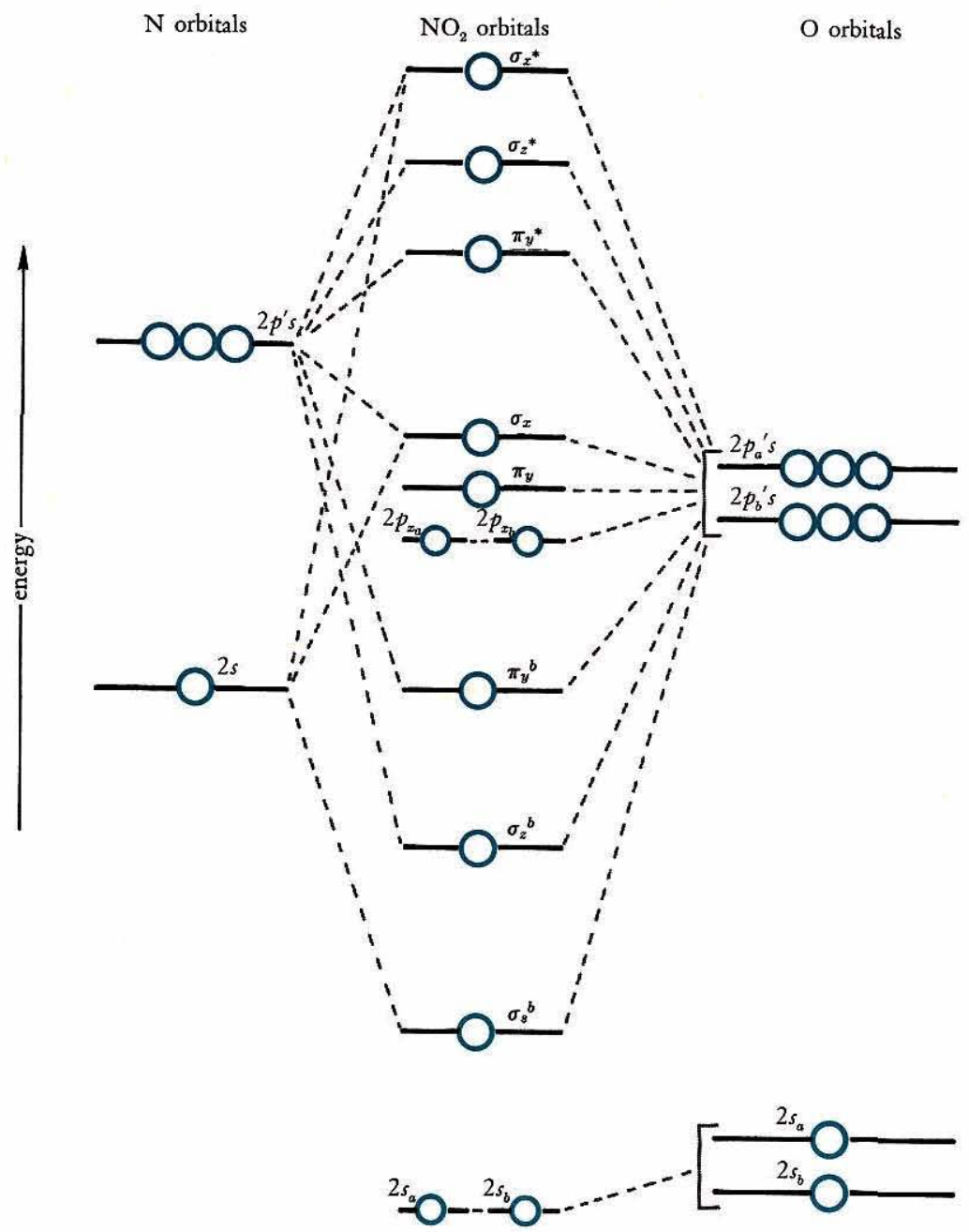

Figure 7-11 Relative orbital energies in $\mathrm{NO}_{2}$. 


\section{7-6 GROUND STATE OF $\mathrm{NO}_{2}$}

There are 17 valence electrons in $\mathrm{NO}_{2}$ (five from nitrogen, six from each oxygen) to place in the molecular orbitals given in Fig. 7-11. The ground state is

$$
\left(2 s_{a}\right)^{2}\left(2 s_{b}\right)^{2}\left(\sigma_{s}^{b}\right)^{2}\left(\sigma_{z}^{b}\right)^{2}\left(\pi_{y}^{b}\right)^{2}\left(2 p_{x_{a}}\right)^{2}\left(2 p_{x_{b}}\right)^{2}\left(\pi_{y}\right)^{2}\left(\sigma_{x}\right) \quad S=\frac{1}{2}
$$

Since there is one unpaired electron, the $\mathrm{NO}_{2}$ molecule is paramagnetic. Electron-spin resonance measurements have confirmed that the unpaired electron in the ground state of $\mathrm{NO}_{2}$ is in a $\sigma$ orbital. The ground-state electronic configuration gives two $\sigma$ bonds and one $\pi$ bond. It is instructive to compare the molecular-orbital bonding scheme with two possible equivalent valence-bond structures that can be written for $\mathrm{NO}_{2}$ (see Fig. 7-12). The resonance between structures I and II spreads out the one $\pi$ bond over the three atoms, an analogy to the $\pi$ bonding molecular orbital (see Fig. 7-10). The unpaired electron is in an $s p^{2}$ hybrid orbital, which is similar to $\sigma_{x}$. The lone pair in the $2 p_{y}$ system goes from $\mathrm{O}_{a}$ to $\mathrm{O}_{b}$, an analogy to the two electrons in the $\pi_{y}$ molecular orbital (see Fig. 7-10).

The $\mathrm{N}-\mathrm{O}$ bond length in $\mathrm{NO}_{2}$ is $1.20 \mathrm{~A}$. This compares with an $\mathrm{N}-\mathrm{O}$ distance of $1.13 \mathrm{~A}$ in NO. The molecular-orbital bonding
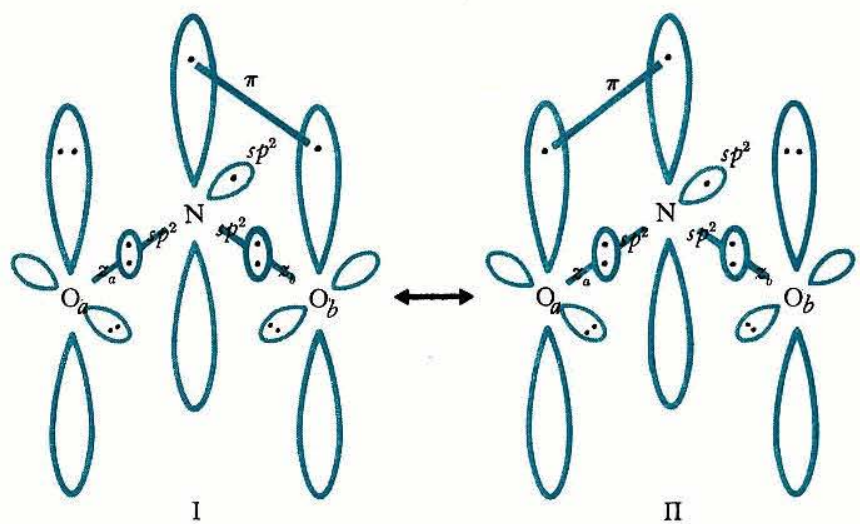

II

Figure 7-12 Valence-bond structures for $\mathrm{NO}_{2}$. 
Table 7-2

Properties of Angular Triatomic Molecules ${ }^{a}$

\begin{tabular}{|c|c|c|c|c|}
\hline $\begin{array}{c}A B_{2} \\
\text { molecule }\end{array}$ & $\begin{array}{c}B-A-B \\
\text { angle, deg }\end{array}$ & Bond & $\begin{array}{l}\text { Bond } \\
\text { length, } A\end{array}$ & $\begin{array}{l}\text { Bond energies } \\
\text { kcal/mole }\end{array}$ \\
\hline $\mathrm{H}_{2} \mathrm{O}$ & 105 & $\begin{array}{r}\mathrm{HO}-\mathrm{H} \\
\mathrm{O}-\mathrm{H}\end{array}$ & 0.958 & $\begin{array}{l}117.5(\mathrm{DE}) \\
110.6(\mathrm{BE})\end{array}$ \\
\hline $\mathrm{H}_{2} \mathrm{~S}$ & 92 & $\begin{array}{l}\mathrm{H}-\mathrm{SH} \\
\mathrm{H}-\mathrm{S}\end{array}$ & 1.334 & $\begin{array}{l}90(\mathrm{DE}) \\
83(\mathrm{BE})\end{array}$ \\
\hline $\mathrm{H}_{2} \mathrm{Se}$ & 91 & $\mathrm{H}-\mathrm{Se}$ & 1.47 & $66(\mathrm{BE})$ \\
\hline $\mathrm{H}_{2} \mathrm{Te}$ & 90 & $\mathrm{H}-\mathrm{Te}$ & & $57(\mathrm{BE})$ \\
\hline $\mathrm{HOCl}$ & 113 & $\mathrm{HO}-\mathrm{Cl}$ & & $60(\mathrm{DE})$ \\
\hline $\mathrm{HOBr}$ & & $\mathrm{HO}-\mathrm{Br}$ & & $56(\mathrm{DE})$ \\
\hline HOI & & $\mathrm{HO}-\mathrm{I}$ & & $56(\mathrm{DE})$ \\
\hline $\mathrm{OF}_{2}$ & 102 & $\mathrm{O}-\mathrm{F}$ & 1.41 & $45.3(\mathrm{BE})$ \\
\hline $\mathrm{OCl}_{2}$ & 115 & $\mathrm{O}-\mathrm{Cl}$ & 1.68 & $49(\mathrm{BE})$ \\
\hline $\mathrm{ClO}_{2}$ & 117 & $\begin{array}{r}\mathrm{OCl}-\mathrm{O} \\
\mathrm{Cl}-\mathrm{O}\end{array}$ & 1.484 & $\begin{array}{l}57(\mathrm{DE}) \\
60(\mathrm{BE})\end{array}$ \\
\hline $\mathrm{BrO}_{2}$ & & $\begin{array}{l}\mathrm{O}-\mathrm{BrO} \\
\mathrm{Br}-\mathrm{O}\end{array}$ & & $\begin{array}{l}70(\mathrm{DE}) \\
60(\mathrm{BE})\end{array}$ \\
\hline $\mathrm{NO}_{2}$ & 132 & $\mathrm{O}-\mathrm{NO}$ & 1.20 & $72(\mathrm{DE})$ \\
\hline NOCl & 116 & $\mathrm{Cl}-\mathrm{NO}$ & 1.95 & $37(\mathrm{DE})$ \\
\hline $\mathrm{NOBr}$ & 117 & $\mathrm{Br}-\mathrm{NO}$ & 2.14 & $28(\mathrm{DE})$ \\
\hline $\mathrm{SO}_{2}$ & 120 & $\mathrm{~s}-\mathrm{O}$ & 1.43 & $119(\mathrm{BE})$ \\
\hline $\mathrm{SeCl}_{2}$ & & $\mathrm{Se}-\mathrm{Cl}$ & & $58(\mathrm{BE})$ \\
\hline $\mathrm{O}_{3}$ & 117 & $\mathrm{O}-\mathrm{O}$ & 1.278 & \\
\hline $\mathrm{NO}_{2}^{-}$ & 115 & $\mathrm{~N}-\mathrm{O}$ & 1.24 & \\
\hline
\end{tabular}

a Data from T. L. Cottrell, The Strengths of Chemical Bonds, Butterworths, London, 1958, Table 11.5.1; L. E. Sutton (ed.), "Interatomic Distances," Special Publication No. 11, The Chemical Society, London, 1958. 
scheme predicts $1 \frac{1}{2} \pi$ bonds for $\mathrm{NO}$, and only $\frac{1}{2}$ for the NO in $\mathrm{NO}_{2}$; thus a longer $\mathrm{NO}$ bond in $\mathrm{NO}_{2}$ is expected. The $\mathrm{O}-\mathrm{NO}$ bond-dissociation energy is $72 \mathrm{kcal} /$ mole.

Bond properties for a number of angular triatomic molecules are given in Table $7-2$.

\section{SUPPLEMENTARY PROBLEMS}

1. Describe the electronic structures of the following molecules: (a) $\mathrm{O}_{3}$; (b) $\mathrm{ClO}_{2}$; (c) $\mathrm{ClO}_{2}+$; (d) $\mathrm{OF}_{2}$.

2. What structure would you expect for the amide ion? for $\mathrm{SCl}_{2}$ ? $\mathrm{XeF}_{2}$ ? 


\section{VIII}

\section{Bonding in Organic Molecules}

\section{8-1 INTRODUCTION}

Carbon atoms have a remarkable ability to form bonds with hydrogen atoms and other carbon atoms. Since carbon has one $2 s$ and three $2 p$ valence orbitals, the structure around carbon for full $\sigma$ bonding is tetrahedral $\left(s p^{3}\right)$. We discussed the bonding in $\mathrm{CH}_{4}$, a simple tetrahedral molecule, in Chapter $\mathrm{V}$. By replacing one hydrogen in $\mathrm{CH}_{4}$ with a $-\mathrm{CH}_{3}$ group, the $\mathrm{C}_{2} \mathrm{H}_{6}$ (ethane) molecule is obtained. The $\mathrm{C}_{2} \mathrm{H}_{6}$ molecule contains one $\mathrm{C}-\mathrm{C}$ bond, and the structure around each carbon is tetrahedral $\left(s p^{3}\right)$, as shown in Fig. 8-1. By continually replacing hydrogens with $-\mathrm{CH}_{3}$ groups, the many bydrocarbons with the full $s p^{3} \sigma$-bonding structure at each carbon are obtained.

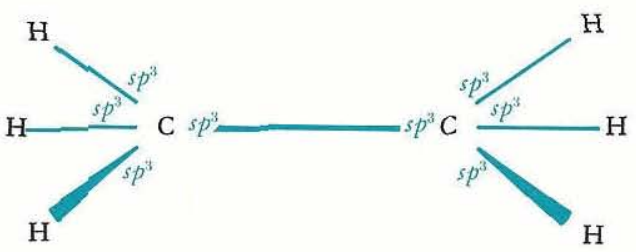

Figure 8-1 Valence-bond structure for $\mathrm{C}_{2} \mathrm{H}_{6}$. 
In many organic molecules, carbon uses only three or two of its four valence orbitals for $\sigma$ bonding. This leaves one or two $2 p$ orbitals for $\pi$ bonding. The main purpose of this chapter is to describe bonding in some of the important atomic groupings containing carbon with $\pi$ valence orbitals.

It is common practice to describe the $\sigma$ bonding of carbon in organic molecules in terms of the hybrid-orbital picture summarized in Table 8-1. The $\pi$ bonding will be described in terms of molecular orbitals, and the energy-level schemes will refer only to the energies of the $\pi$ molecular orbitals. This is a useful way of handling the electronic energy levels, since the $\sigma$ bonding orbitals are usually considerably more stable than the $\pi$ bonding orbitals. Thus the chemically and spectroscopically "active" electrons reside in the $\pi$ molecular orbitals.

\section{8-2 $\quad \mathrm{C}_{2} \mathrm{H}_{4}$}

The structure of ethylene, $\mathrm{C}_{2} \mathrm{H}_{4}$, is shown in Fig. 8-2. The molecule is planar, and each carbon is bonded to two hydrogens and to the other carbon. With three groups attached to each carbon, we use a set of $s p^{2}$ hybrid orbitals for $\sigma$ bonding.

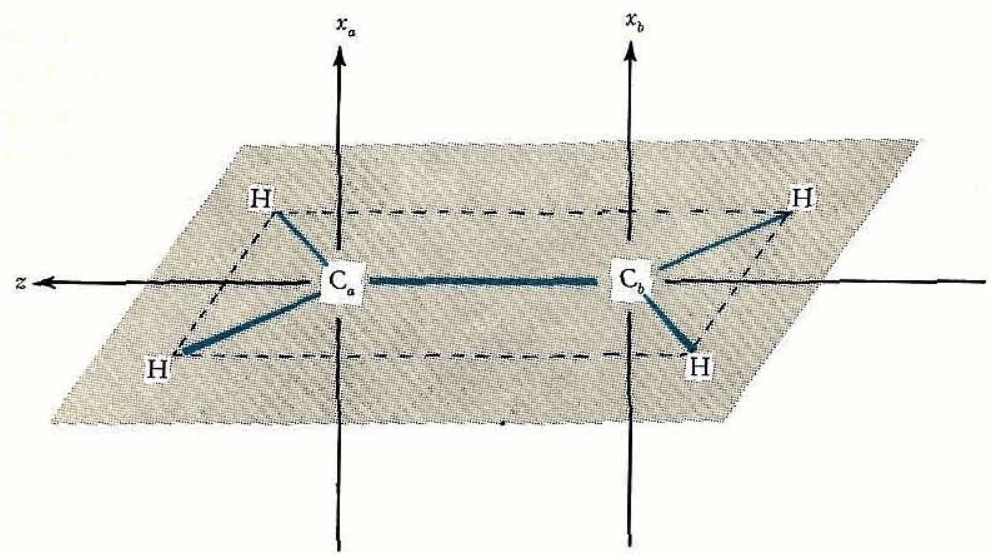

Figure 8-2 Coordinate system for $\mathrm{C}_{2} \mathrm{H}_{4}$. 
Table 8-1

Hybrid-Orbital Picture for $\sigma$ Bonding of Carbon in Organic Molecules

\begin{tabular}{ccl}
$\begin{array}{c}\text { Number of atoms } \\
\text { bound to carbon }\end{array}$ & $\begin{array}{c}\text { o Bond } \\
\text { orbitals }\end{array}$ & $\begin{array}{c}\text { Structure } \\
\text { around carbon }\end{array}$ \\
\hline 4 & $s p^{3}$ & tetrahedral \\
3 & $s p^{2}$ & trigonal planar \\
2 & $s p$ & linear \\
\hline
\end{tabular}
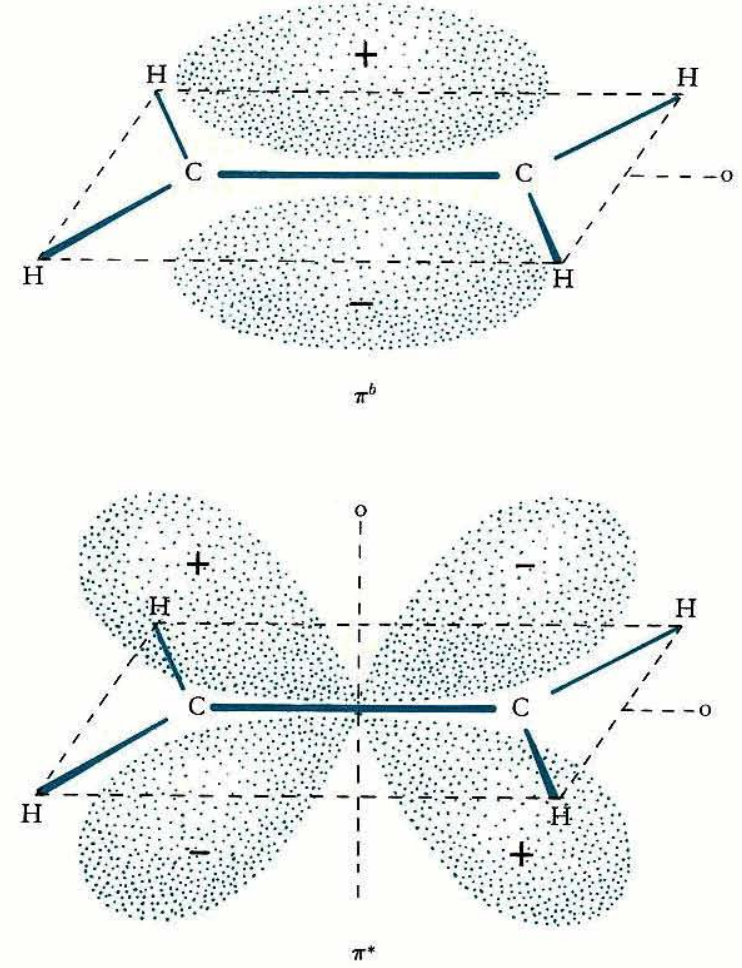

Figure 8-3 Boundary surfaces of the $\pi$ molecular orbitals of $\mathbf{C}_{2} \mathbf{H}_{4}$. 
This leaves each carbon with a $2 p$ orbital, which is perpendicular to the plane of the molecule. We form bonding and antibonding molecular orbitals with the $2 p_{x}$ valence orbitals, as follows:

$$
\begin{aligned}
& \psi\left(\pi^{b}\right)=\frac{1}{\sqrt{2}}\left(x_{a}+x_{b}\right) \\
& \psi\left(\pi^{*}\right)=\frac{1}{\sqrt{2}}\left(x_{a}-x_{b}\right)
\end{aligned}
$$

The boundary surfaces of the $\pi^{b}$ and $\pi^{*}$ MO's are shown in Fig. 8-3.

\section{8-3 energy levels in $\mathrm{C}_{2} \mathrm{H}_{4}$}

The energies of the $\pi^{b}$ and $\pi^{*}$ MO's are obtained just as were the energies of the $\sigma^{b}$ and $\sigma^{*} \mathrm{MO}^{\prime}$ 's of $\mathrm{H}_{2}$ (Section 2-4):

$$
\begin{aligned}
& E\left[\psi\left(\pi^{b}\right)\right]=\int \psi\left(\pi^{b}\right) \mathcal{H} \psi\left(\pi^{b}\right) d \tau=\frac{1}{2} \int\left(x_{a}+x_{b}\right) \mathfrak{H}\left(x_{a}+x_{b}\right) d \tau \\
& =q_{c}+\beta_{c c} \\
& E\left[\psi\left(\pi^{*}\right)\right]=\frac{1}{2} \int\left(x_{a}-x_{b}\right) \mathcal{H C}\left(x_{a}-x_{b}\right) d \tau=q_{c}-\beta_{c c}
\end{aligned}
$$

Thus we have the same type of energy-level scheme for the $\pi$ molecular orbitals of ethylene as we had for the $\sigma$ molecular orbitals of the hydrogen molecule. The diagram for $\mathrm{C}_{2} \mathrm{H}_{4}$ is shown in Fig. 8-4.

\section{8-4 Ground state of $\mathrm{C}_{2} \mathrm{H}_{4}$}

There are twelve valence electrons in $\mathrm{C}_{2} \mathrm{H}_{4}$, eight from the two carbons $\left(2 s^{2} 2 p^{2}\right)$ and one from each hydrogen. Ten of these electrons are used in $\sigma$ bonding, as shown in Fig. 8-5. Two electrons are left 
to place in the $\pi$ molecular orbitals. The ground state is $\left(\pi^{b}\right)^{2}$, which gives one $\pi$ bond. The usual pictures of the bonding in $\mathrm{C}_{2} \mathrm{H}_{4}$ are shown in Fig. 8-6.

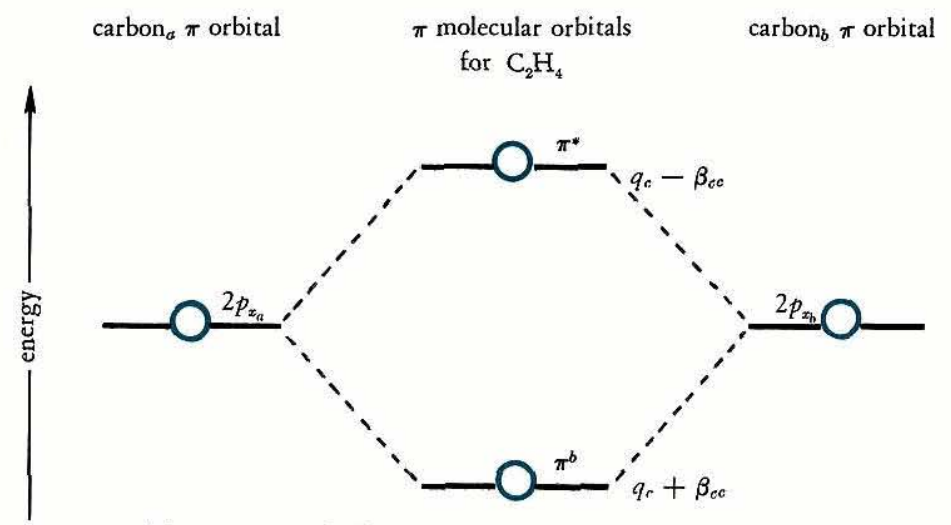

Figure 8-4 Relative $\pi$ orbital energies in $\mathrm{C}_{2} \mathrm{H}_{4}$.

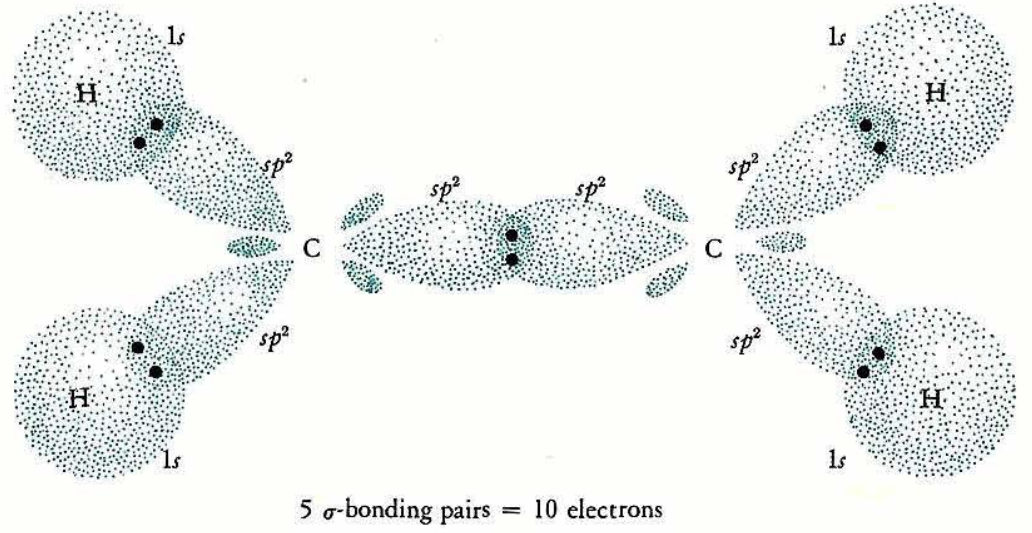

Figure 8-5 The $\sigma$ bonding structure of $\mathrm{C}_{2} \mathrm{H}_{4}$. 


\section{8-5 BENT-BOND PICTURE OF $\mathrm{C}_{2} \mathrm{H}_{4}$}

The $\mathrm{C}=\mathrm{C}$ bond can be formulated as involving two equivalent "bent" bonds, rather than one $\sigma$ and one $\pi$ bond. One simple way to construct equivalent bent bonds is to linearly combine the $\sigma^{b}$ and $\pi^{b}$ molecular orbitals of $\mathrm{C}_{2} \mathrm{H}_{4}$ as follows:

$$
\begin{aligned}
& \psi_{1}=\frac{1}{\sqrt{2}}\left[\psi\left(\sigma_{c c}{ }^{b}\right)+\psi\left(\pi_{c c^{b}}{ }^{b}\right)\right] \\
& \psi_{2}=\frac{1}{\sqrt{2}}\left[\psi\left(\sigma_{c c^{b}}{ }^{b}\right)-\psi\left(\pi_{c c}^{b}\right)\right]
\end{aligned}
$$

The equivalent orbitals $\psi_{1}$ and $\psi_{2}$ are shown in Fig. 8-7. If the $\sigma^{b}$ orbitals used are derived from carbon $s p^{2}$ orbitals (Section 8-2), the $\mathrm{H}-\mathrm{C}-\mathrm{H}$ and $\mathrm{H}-\mathrm{C}-\mathrm{C}$ bond angles should be $120^{\circ}$.

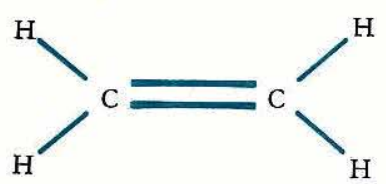

(a) simple picture

lines indicate electron-pair bonds

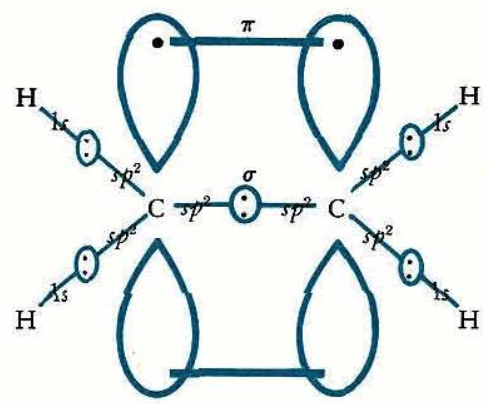

(b) $\sigma-\pi$ bond orbital picture

Figure 8-6 Common representations of the bonding in $\mathrm{C}_{2} \mathrm{H}_{4}$. 
Using only valence-bond ideas, we can formulate the bonding in $\mathrm{C}_{2} \mathrm{H}_{4}$ as involving four $s p^{3}$ orbitals on each carbon. Two of the $s p^{3}$ orbitals are used to attach two hydrogens, and two are used to bond to the other carbon in the double bond. Thus, $\mathrm{C}_{2} \mathrm{H}_{4}$ would be represented as shown in Fig. 8-8. This model predicts an $\mathrm{H}-\mathrm{C}-\mathrm{H}$ angle of $109^{\circ} 28^{\prime}$ and an $\mathrm{H}-\mathrm{C}=\mathrm{C}$ angle of $125^{\circ} 16^{\prime}$.

The observed $\mathrm{H}-\mathrm{C}-\mathrm{H}$ angle in $\mathrm{C}_{2} \mathrm{H}_{4}$ is $117^{\circ}$. Since the molecule is planar, the $\mathrm{H}-\mathrm{C}=\mathrm{C}$ angle is $121.5^{\circ}$. These angles are much closer in size to the $120^{\circ}$ angle between equivalent $s p^{2}$ hybrid orbitals than they are to the tetrahedral hybrid-orbital predictions. However, certain other molecules containing the $\mathrm{C}=\mathrm{C}$ group have $\mathrm{X}-\mathrm{C}=\mathrm{C}$
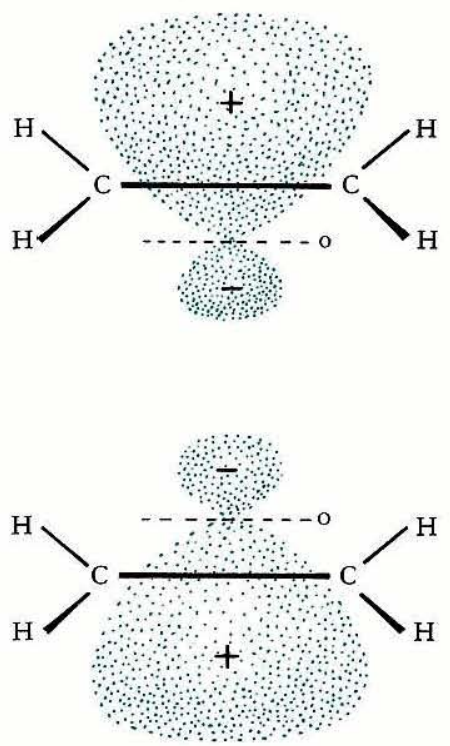

Figure 8-7 Equivalent orbitals in $\mathrm{C}_{2} \mathrm{H}_{4}$, constructed from the $\sigma^{b}$ and $\pi^{b}$ orbitals. 


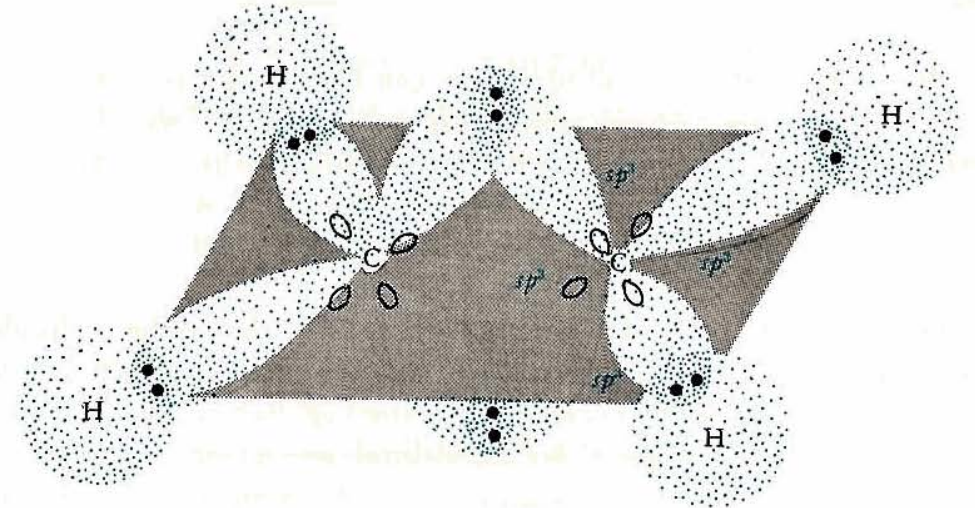

Figure 8-8 Equivalent orbitals in $\mathrm{C}_{2} \mathrm{H}_{4}$, using $\boldsymbol{s p}^{3}$ orbitals on each carbon.

angles in the neighborhood of $125^{\circ}$.

The multiple bonds in molecules such as $\mathrm{N}_{2}, \mathrm{H}_{2} \mathrm{CO}$, and $\mathrm{C}_{2} \mathrm{H}_{2}$ can be formulated either as equivalent bent bonds or as a combination of $\sigma$ and $\pi$ bonds. For a more complete discussion of equivalent orbitals, the reader is referred elsewhere. ${ }^{1}$

\section{8-6 BOND PROPERTIES OF THE $\mathrm{C}=\mathrm{C}$ GROUP}

There are two kinds of bonds in $\mathrm{C}_{2} \mathrm{H}_{4}, \mathrm{C}=\mathrm{C}$ and $\mathrm{C}-\mathrm{H}$. Thus we must know the value of $\mathrm{BE}(\mathrm{C}-\mathrm{H})$ in order to obtain the value of $\mathrm{BE}(\mathrm{C}=\mathrm{C})$ from the process

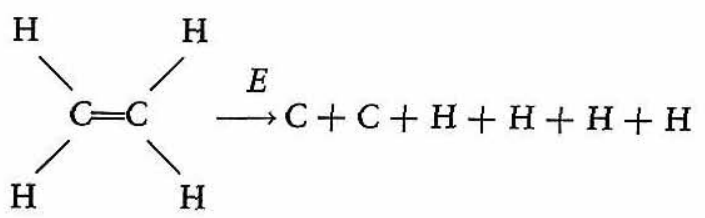

${ }^{1}$ J. A. Pople, Quart. Rev., XI, 273 (1957); L. Pauling, Nature of the Chemical Bond, Cornell University Press, Ithaca, N.Y., 1960, p. $138 \mathrm{ff}$. 
The value of $\mathrm{BE}(\mathrm{C}-\mathrm{H})$ used to calculate bond energies such as $\mathrm{C}=\mathrm{C}$, $\mathrm{C}=\mathrm{O}$, etc., is $98.7 \mathrm{kcal} / \mathrm{mole}$, which is very nearly the $\mathrm{BE}(\mathrm{C}-\mathrm{H})$ in $\mathrm{CH}_{4}$. Bond energies and bond lengths for a number of important groups are given in Table 8-2. The values are averaged from several compounds unless otherwise indicated.

The average $\mathrm{C}=\mathrm{C}$ bond energy is $145.8 \mathrm{kcal} / \mathrm{mole}$, a value almost twice as large as the $\mathrm{C}-\mathrm{C}$ bond energy of $82.6 \mathrm{kcal} / \mathrm{mole}$. The $\mathrm{C}=\mathrm{C}$ bond length is $1.35 \mathrm{~A}$, which is shorter than the $1.54 \mathrm{~A} \mathrm{C}-\mathrm{C}$ bond distance.

Table $8-2$

Bond Properties of Organic Groups ${ }^{a}$

\begin{tabular}{|c|c|c|}
\hline Bond & $\begin{array}{c}\text { Bond } \\
\text { length, } A\end{array}$ & $\begin{array}{l}\text { Bond energy, } \\
\text { kcal/mole }\end{array}$ \\
\hline $\mathrm{C}-\mathrm{H}$ & 1.08 & 98.7 \\
\hline $\mathrm{C}-\mathrm{C}$ & 1.54 & 82.6 \\
\hline $\mathrm{C}=\mathrm{C}$ & 1.35 & 145.8 \\
\hline $\mathrm{C} \equiv \mathrm{C}$ & 1.21 & 199.6 \\
\hline $\mathrm{C}-\mathrm{C}\left(\right.$ in $\mathrm{C}_{2} \mathrm{H}_{6}$ ) & 1.543 & $83(\mathrm{DE})$ \\
\hline $\mathrm{C}=\mathrm{C}\left(\right.$ in $\left.\mathrm{C}_{2} \mathrm{H}_{4}\right)$ & 1.353 & $125(\mathrm{DE}) ; 142.9(\mathrm{BE})$ \\
\hline $\mathrm{C} \equiv \mathrm{C}\left(\right.$ in $\left.\mathrm{C}_{2} \mathrm{H}_{2}\right)$ & 1.207 & $230(\mathrm{DE}) ; 194.3(\mathrm{BE})$ \\
\hline $\mathrm{C}-\mathrm{N}$ & 1.47 & 72.8 \\
\hline $\mathrm{C}=\mathrm{N}$ & & 147 \\
\hline $\mathrm{C} \equiv \mathrm{N}$ & 1.14 & 212.6 \\
\hline $\mathrm{C}-\mathrm{O}$ & 1.43 & 85.5 \\
\hline $\mathrm{C}=\mathrm{O}$ (in aldehydes) & 1.22 & 176 \\
\hline $\mathrm{C}=\mathrm{O}$ (in ketones) & 1.22 & 179 \\
\hline $\mathrm{C}=\mathrm{O}\left(\right.$ in $\left.\mathrm{H}_{2} \mathrm{CO}\right)$ & 1.21 & 166 \\
\hline $\mathrm{C}-\mathrm{F}\left(\right.$ in $\left.\mathrm{CF}_{4}\right)$ & 1.36 & 116 \\
\hline $\mathrm{C}-\mathrm{Si}\left[\mathrm{in} \mathrm{Si}\left(\mathrm{CH}_{3}\right)_{4}\right]$ & 1.93 & 72 \\
\hline $\mathrm{C}-\mathrm{S}\left(\right.$ in $\left.\mathrm{C}_{2} \mathrm{H}_{5} \mathrm{SH}\right)$ & 1.81 & 65 \\
\hline $\mathrm{C}=\mathrm{S}\left(\right.$ in $\left.\mathrm{CS}_{2}\right)$ & 1.55 & 128 \\
\hline $\mathrm{C}-\mathrm{Cl}$ & 1.76 & 81 \\
\hline $\mathrm{C}-\mathrm{Br}$ & $1.94\left(\right.$ in $\left.\mathrm{CH}_{3} \mathrm{Br}\right)$ & $68\left(\right.$ in $\left.\mathrm{C}_{2} \mathrm{H}_{5} \mathrm{Br}\right)$ \\
\hline $\mathrm{C}-\mathrm{I}\left(\right.$ in $\left.\mathrm{CH}_{3} \mathrm{I}\right)$ & 2.14 & 51 \\
\hline
\end{tabular}

${ }^{a}$ Data from T. L. Cottrell, The Strengths of Chemical Bonds, Butterworths, London, 1958, Table 11.5.1. 


\section{8-7 The value of $\beta_{c c}$ IN $\mathrm{C}_{2} \mathrm{H}_{4}$}

The first excited state of $\mathrm{C}_{2} \mathrm{H}_{4}$ occurs upon excitation of an electron from $\pi^{b}$ to $\pi^{*}$, giving the configuration $\left(\pi^{b}\right)\left(\pi^{*}\right)$. We see that the difference in energy between $\pi^{b}$ and $\pi^{*}$ is $-2 \beta$. Absorption of light at the $1650 \mathrm{~A}$ wavelength causes the $\pi^{b} \rightarrow \pi^{*}$ excitation to take place. Since $1650 \mathrm{~A}$ is equal to $60,600 \mathrm{~cm}^{-1}$ or $174 \mathrm{kcal} / \mathrm{mole}$, we have

and

$$
-2 \beta_{c c}=60,600 \mathrm{~cm}^{-1} \text { or } \quad 174 \mathrm{kcal} / \mathrm{mole}
$$

$$
\beta_{c c}=-30,300 \mathrm{~cm}^{-1} \quad \text { or } \quad-87 \mathrm{kcal} / \mathrm{mole}
$$

\section{8-8 $\quad \mathrm{H}_{2} \mathrm{CO}$}

The simplest molecule containing the $\mathrm{C}=\mathrm{O}$ group is formaldehyde, $\mathrm{H}_{2} \mathrm{CO}$. The $\sigma$ bonding in $\mathrm{H}_{2} \mathrm{CO}$ can be represented as involving $s p^{2}$ orbitals on carbon. This leaves one $2 p$ orbital on carbon for $\pi$ bond-

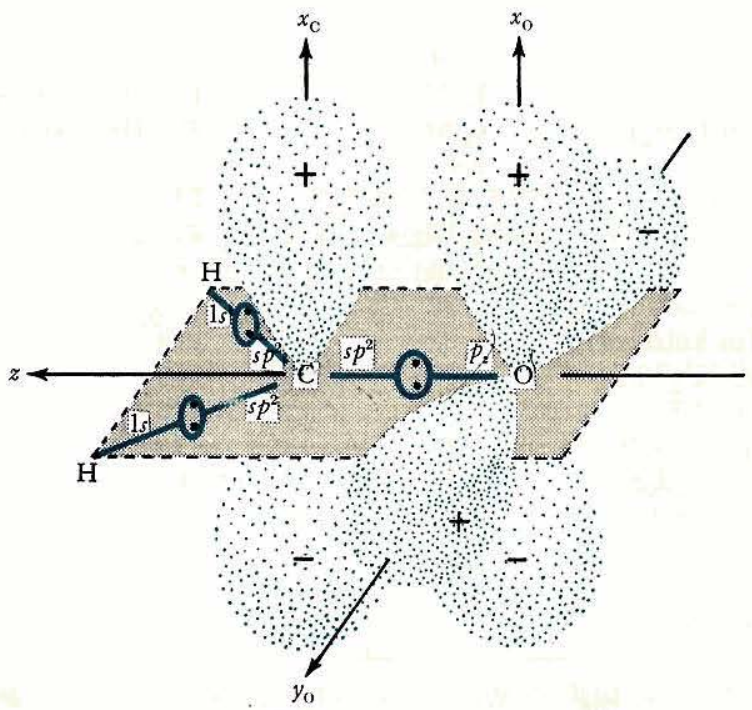

Figure 8-9 Orbitals in the $\mathrm{H}_{2} \mathrm{CO}$ molecule. 
ing to the oxygen, as shown in Fig. 8-9. The $\pi$ molecular orbitals are:

$$
\begin{aligned}
& \psi\left(\pi_{x}^{b}\right)=C_{1} x_{C}+C_{2} x_{0} \\
& \psi\left(\pi_{x}^{*}\right)=C_{3} x_{C}-C_{4} x_{0}
\end{aligned}
$$

Since oxygen is more electronegative than carbon, we expect $\left(C_{2}\right)^{2}>$ $\left(C_{1}\right)^{2}$ and $\left(C_{3}\right)^{2}>\left(C_{4}\right)^{2}$. Since the oxygen $2 p_{z}$ orbital is used in $\sigma$ bonding, we have the $2 p_{y}$ orbital remaining as a nonbonding $\mathrm{MO}$ of the $\pi$ type. The energy-level scheme expected for the $\pi$ molecular orbitals of $\mathrm{H}_{2} \mathrm{CO}$ is shown in Fig. 8-10.

\section{8-9 GRound state of $\mathrm{H}_{2} \mathrm{CO}$}

There are twelve valence electrons in $\mathrm{H}_{2} \mathrm{CO}$, two from the hydrogens, four from carbon, and six from oxygen $\left(2 s^{2} 2 p^{4}\right)$. Six of these electrons are involved in $\sigma$ bonding, and two are in the oxygen $2 s$ orbital as a lone pair. This leaves four electrons for the $\pi$ orbitals shown in Fig. 8-10. The ground state is $\left(\pi_{x}^{b}\right)^{2}\left(\pi_{y}\right)^{2}$. There is one

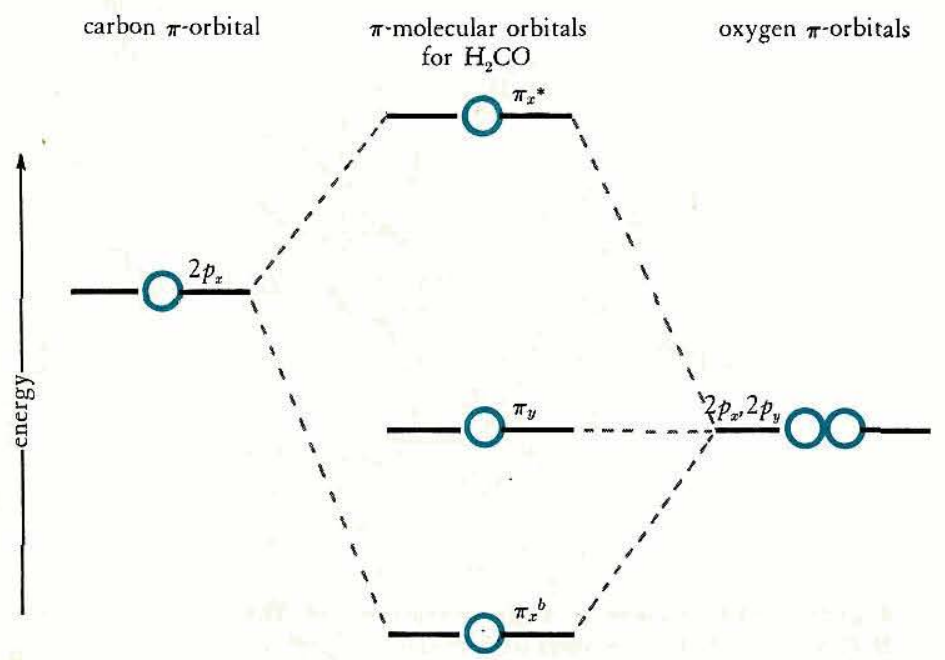

Figure 8-10 Relative $\pi$ orbital energies in $\mathrm{H}_{2} \mathrm{CO}$. 
carbon-oxygen $\pi$ bond, along with the $\sigma$ bond, giving an electronic structure that is commonly represented as shown in Fig. 8-11.

The carbonyl $(\mathrm{C}=\mathrm{O})$ group is present in many classes of organic compounds, among them aldehydes, ketones, esters, acids, and amides. The simplest ketone is acetone, $\left(\mathrm{CH}_{3}\right)_{2} \mathrm{C}=\mathrm{O}$. The $\mathrm{C}=\mathrm{O}$ bond energy in $\mathrm{H}_{2} \mathrm{CO}$ is $166 \mathrm{kcal} / \mathrm{mole}$. As $\mathrm{C}-\mathrm{H}$ bonds are replaced by $\mathrm{C}-\mathrm{C}$ bonds, the $\mathrm{C}=\mathrm{O}$ bond energy increases. The average $\mathrm{C}=\mathrm{O}$ bond energy for aldehydes is $176 \mathrm{kcal} / \mathrm{mole}$; for ketones it is 179 $\mathrm{kcal} / \mathrm{mole}$. Each of these average values is more than twice the 85.5 $\mathrm{kcal} / \mathrm{mole}$ value for the $\mathrm{C}-\mathrm{O}$ bond energy. The average $\mathrm{C}=\mathrm{O}$ bond length is $1.22 \mathrm{~A}$, which lies between $\mathrm{C} \equiv \mathrm{O}(R=1.13 \mathrm{~A})$ and $\mathrm{C}-\mathrm{O}$ $(R=1.43 \mathrm{~A})$.

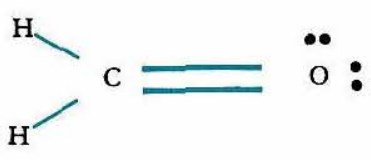

(a)

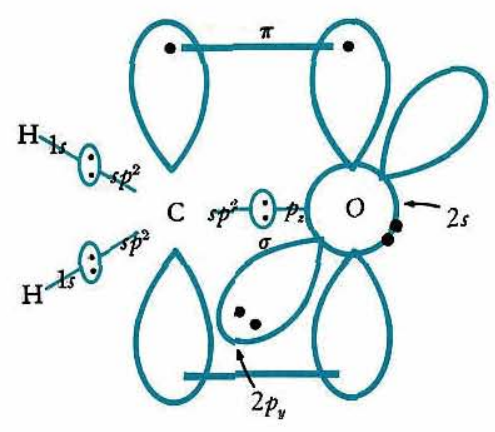

(b)

Figure 8-11 Common representations of the bonding in $\mathrm{H}_{2} \mathrm{CO}$. 


\section{8-10 THE $n \rightarrow \pi^{*}$ TRANSITION EXHIBITED BY THE CARBONYL GROUP}

The excitation of an electron from $\pi_{y}$ to $\pi_{x}{ }^{*}$ occurs with absorption of light in the 2700-3000 A wavelength region. Thus the carbonyl group exhibits a very characteristic absorption spectrum. Since the transition is from a nonbonding $\pi$ orbital to an antibonding $\pi$ orbital, it is commonly called an $n \rightarrow \pi^{*}$ transition.

\section{8-11 $\mathrm{C}_{2} \mathrm{H}_{2}$}

The structure of acetylene, $\mathrm{C}_{2} \mathrm{H}_{2}$, is shown in Fig. 8-12. The $\sigma$ bonding involves $s p$ hybrid orbitals on the carbons, leaving each carbon with two mutually perpendicular $2 p$ orbitals for $\pi$ bonding. The $\pi$ molecular orbitals are the same as those for a homonuclear diatomic molecule:

$$
\begin{aligned}
& \psi\left(\pi_{x}^{b}\right)=\frac{1}{\sqrt{2}}\left(x_{a}+x_{b}\right) \\
& \psi\left(\pi_{y}^{b}\right)=\frac{1}{\sqrt{2}}\left(y_{a}+y_{b}\right) \\
& \psi\left(\pi_{x}^{*}\right)=\frac{1}{\sqrt{2}}\left(x_{a}-x_{b}\right)
\end{aligned}
$$

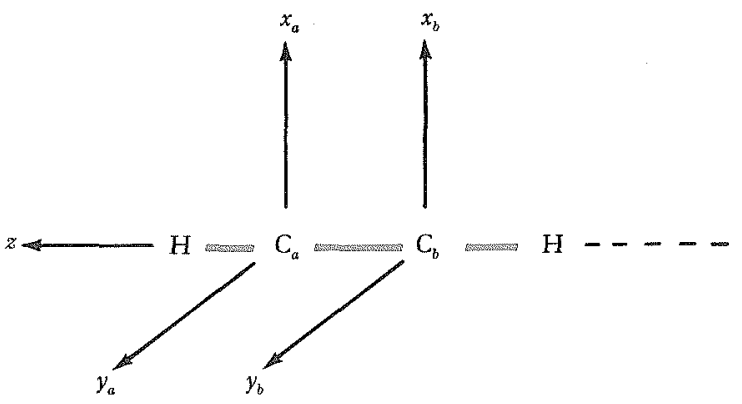

Figure 8-12 Coordinate system for $\mathrm{C}_{2} \mathrm{H}_{2}$. 


$$
\psi\left(\pi_{y}^{*}\right)=\frac{1}{\sqrt{2}}\left(y_{a}-y_{b}\right)
$$

The energies of the $\pi$ molecular orbitals are shown in Fig. 8-13.

\section{8-12 Ground state of $\mathrm{C}_{2} \mathrm{H}_{2}$}

There are ten valence electrons in $\mathrm{C}_{2} \mathrm{H}_{2}$. Six are required for $\sigma$ bonding, and the other four give a ground state $\left(\pi_{x}^{b}\right)^{2}\left(\pi_{y}^{b}\right)^{2}$. Thus we have three carbon-carbon bonds, one $\sigma$ bond, and two $\pi$ bonds. The common bonding pictures for $\mathrm{C}_{2} \mathrm{H}_{2}$ are shown in Fig. 8-14.

The bond energy of the $\mathrm{C} \equiv \mathrm{C}$ group, $199.6 \mathrm{kcal} / \mathrm{mole}$, is larger than that of $\mathrm{C}-\mathrm{C}$ or $\mathrm{C}=\mathrm{C}$, but smaller than that of $\mathrm{C} \equiv \mathrm{O}$. The $\mathrm{C} \equiv \mathrm{C}$ bond length is $1.21 \mathrm{~A}$, shorter than either $\mathrm{C}=\mathrm{C}$ or $\mathrm{C}-\mathrm{C}$.

\section{8-13 $\mathrm{CH}_{3} \mathrm{CN}$}

The nitrile group, $\mathrm{C} \equiv \mathrm{N}$, is another important functional group in organic chemistry. The simple compound $\mathrm{CH}_{3} \mathrm{CN}$ is called acetonitrile; its structure is shown in Fig. 8-15. The $\pi$ bonding in the $\mathrm{C} \equiv \mathrm{N}$ group is very similar to the $\pi$ bonding in $\mathrm{C} \equiv \mathrm{C}$. The usual bonding pictures are also shown in Fig. 8-15.

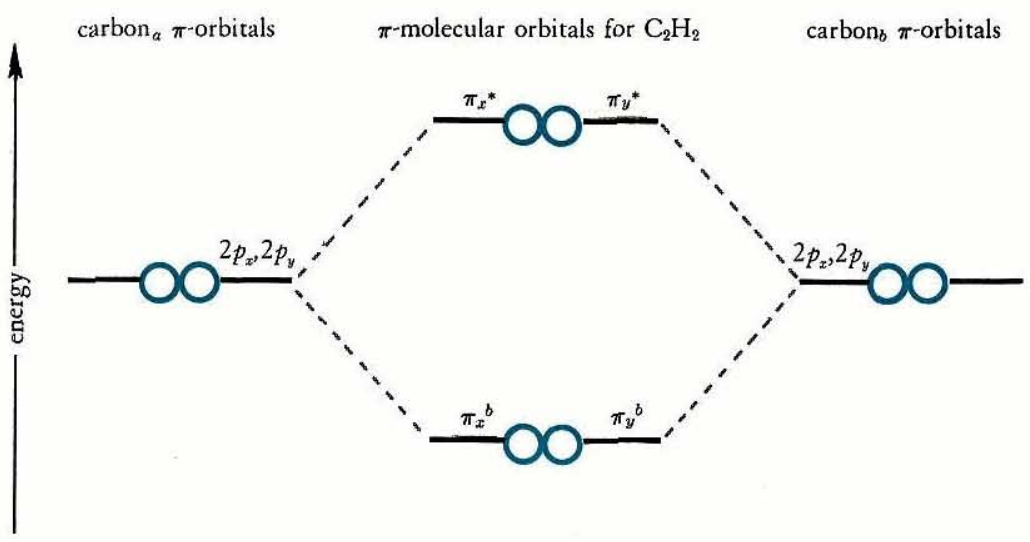

Figure 8-13 Relative orbital energies in $\mathrm{C}_{2} \mathrm{H}_{2}$. 
Bonding in Organic Molecules

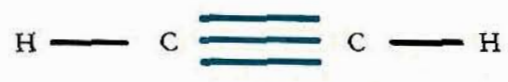

(a)

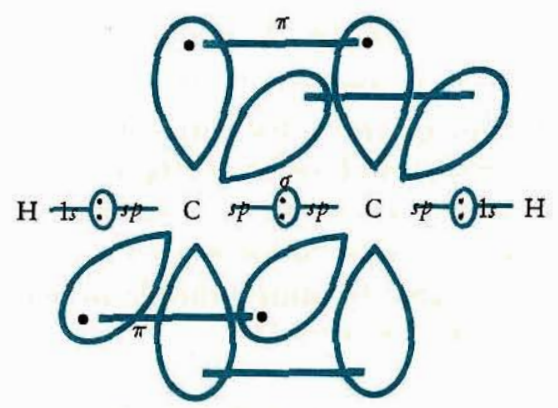

(b)

Figure 8-14 Common representations of the bonding in $\mathrm{C}_{2} \mathrm{H}_{2}$.
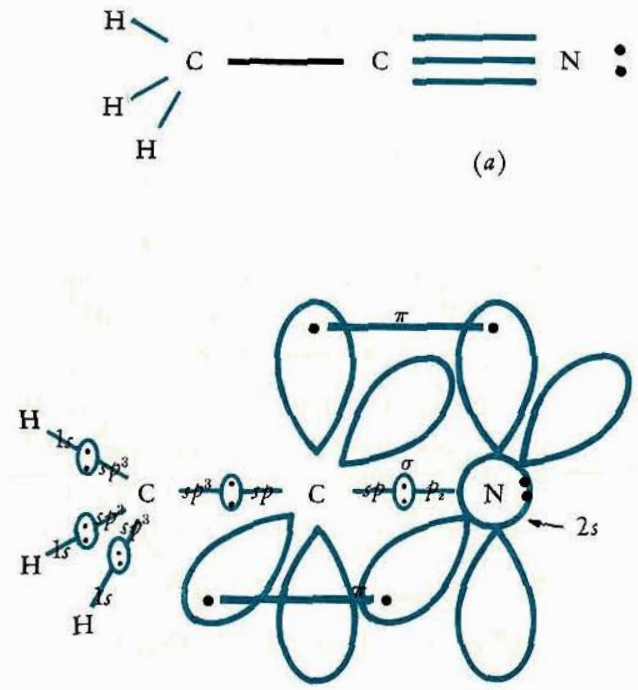

(b)

Figure 8-15 Common representations of the bonding in $\mathrm{CH}_{3} \mathrm{CN}$. 
The $\mathrm{C} \equiv \mathrm{N}$ bond energy, $212.6 \mathrm{kcal} / \mathrm{mole}$, is larger than that of $\mathrm{C} \equiv \mathrm{C}$. The $\mathrm{C} \equiv \mathrm{N}$ bond length is about $1.14 \mathrm{~A}$.

\section{8-14 $\mathrm{C}_{6} \mathrm{H}_{6}$}

The planar structure of benzene $\left(\mathrm{C}_{6} \mathrm{H}_{6}\right)$ is shown in Fig. 8-16. Each carbon is bonded to two other carbons and to one hydrogen. Thus we use $s p^{2}$ hybrid orbitals on the carbons for $\sigma$ bonding. Each carbon has a $2 p$ orbital for $\pi$ bonding, also shown in Fig. 8-16. With $\operatorname{six} \pi$ valence orbitals, we need to construct six $\pi$ molecular orbitals for $\mathrm{C}_{6} \mathrm{H}_{6}$. The most stable bonding orbital concentrates electronic density between each pair of nuclei:

$$
\psi\left(\pi_{1}^{b}\right)=\frac{1}{\sqrt{6}}\left(z_{a}+z_{b}+z_{c}+z_{d}+z_{e}+z_{f}\right)
$$

The least stable antibonding orbital has nodes between the nuclei:

$$
\psi\left(\pi_{3}^{*}\right)=\frac{1}{\sqrt{6}}\left(z_{a}-z_{b}+z_{c}-z_{d}+z_{e}-z_{f}\right)
$$

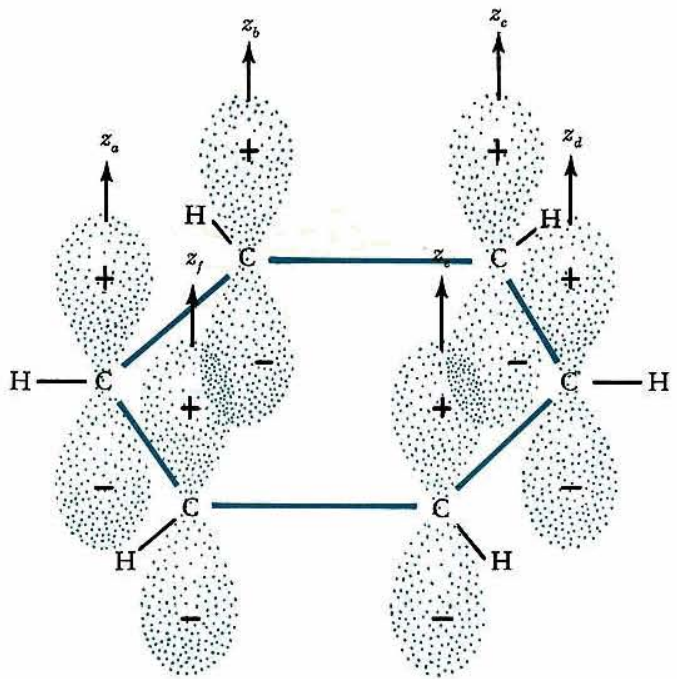

Figure 8-16 Structure and the $\pi$ valence orbitals of $\mathrm{C}_{6} \mathrm{H}_{6}$. 
The other molecular orbitals ${ }^{1}$ have energies between $\pi^{b}$ and $\pi^{*}$ :

$$
\begin{aligned}
& \psi\left(\pi_{2}^{b}\right)=\frac{1}{2 \sqrt{3}}\left(2 z_{a}+z_{b}-z_{c}-2 z_{d}-z_{e}+z_{j}\right) \\
& \psi\left(\pi_{3}^{b}\right)=\frac{1}{2}\left(z_{a}+z_{b}-z_{d}-z_{e}\right) \\
& \psi\left(\pi_{1}^{*}\right)=\frac{1}{2 \sqrt{3}}\left(2 z_{a}-z_{b}-z_{c}+2 z_{d}-z_{e}-z_{f}\right) \\
& \psi\left(\pi_{2}^{*}\right)=\frac{1}{2}\left(z_{a}-z_{b}+z_{d}-z_{e}\right)
\end{aligned}
$$

The molecular orbitals for benzene are shown in Fig. 8-17.

\section{8-15 Molecular-orbital energies in $\mathrm{C}_{6} \mathrm{H}_{6}$}

The most stable orbital in benzene is $\psi\left(\pi_{1}^{b}\right)$. The energy of this MO is calculated below:

$$
\begin{aligned}
& E\left[\psi\left(\pi_{1}^{b}\right)\right]=\int \mathcal{H}\left(\pi_{1}^{b}\right) \mathcal{H} \psi\left(\pi_{1}^{b}\right) d \tau \\
& =\frac{1}{6} \mathcal{S}\left(z_{a}+z_{b}+z_{c}+z_{d}+z_{e}+z_{f}\right) \mathcal{H} \\
& \times\left(z_{a}+z_{b}+z_{c}+z_{d}+z_{e}+z_{f}\right) d \tau \\
& =\frac{1}{6}\left[6 q_{c}+12 \beta_{c c}+2 \int Z_{a} \mathfrak{F} z_{c} d \tau+2 \int z_{a} \mathfrak{C}_{z_{d}} d \tau+2 \int_{z_{a}} \mathfrak{F} z_{e}\right. \\
& \times d \tau+2 \int z_{b} \mathfrak{H} z_{d} d \tau+2 \int z_{b} \mathcal{H C}_{e} d \tau+2 \int z_{b} \mathcal{H C}_{f} d \tau
\end{aligned}
$$

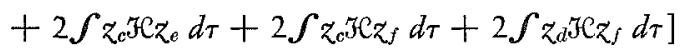

In other words, on expansion of the integral, we obtain six coulomb integrals (such as $\int z_{a} T C z_{a} d \tau$ ) and twelve exchange integrals involving adjacent $p$ orbitals (such as $\int_{z_{a}} \mathcal{H} Z_{b} d \tau$ ); the other integrals are exchange integrals involving nonadjacent $p$ orbitals (such as $\left.\int z_{a} \mathfrak{H} z_{c} d \tau\right)$. We expect these integrals to be much smaller than the regular $\beta$ 's. If we adopt the frequently used Hückel approximation in which such integrals are taken to be zero, we have

$$
E\left[\psi\left(\pi_{1}^{b}\right)\right]=q_{c}+2 \beta_{c c}
$$

The energy-level scheme for $\mathrm{C}_{6} \mathrm{H}_{6}$ is shown in Fig. 8-18.

1 The rules for constructing the benzene molecular orbitals are straightforward, but require symmetry and orthogonality principles that have not been presented in this book. 

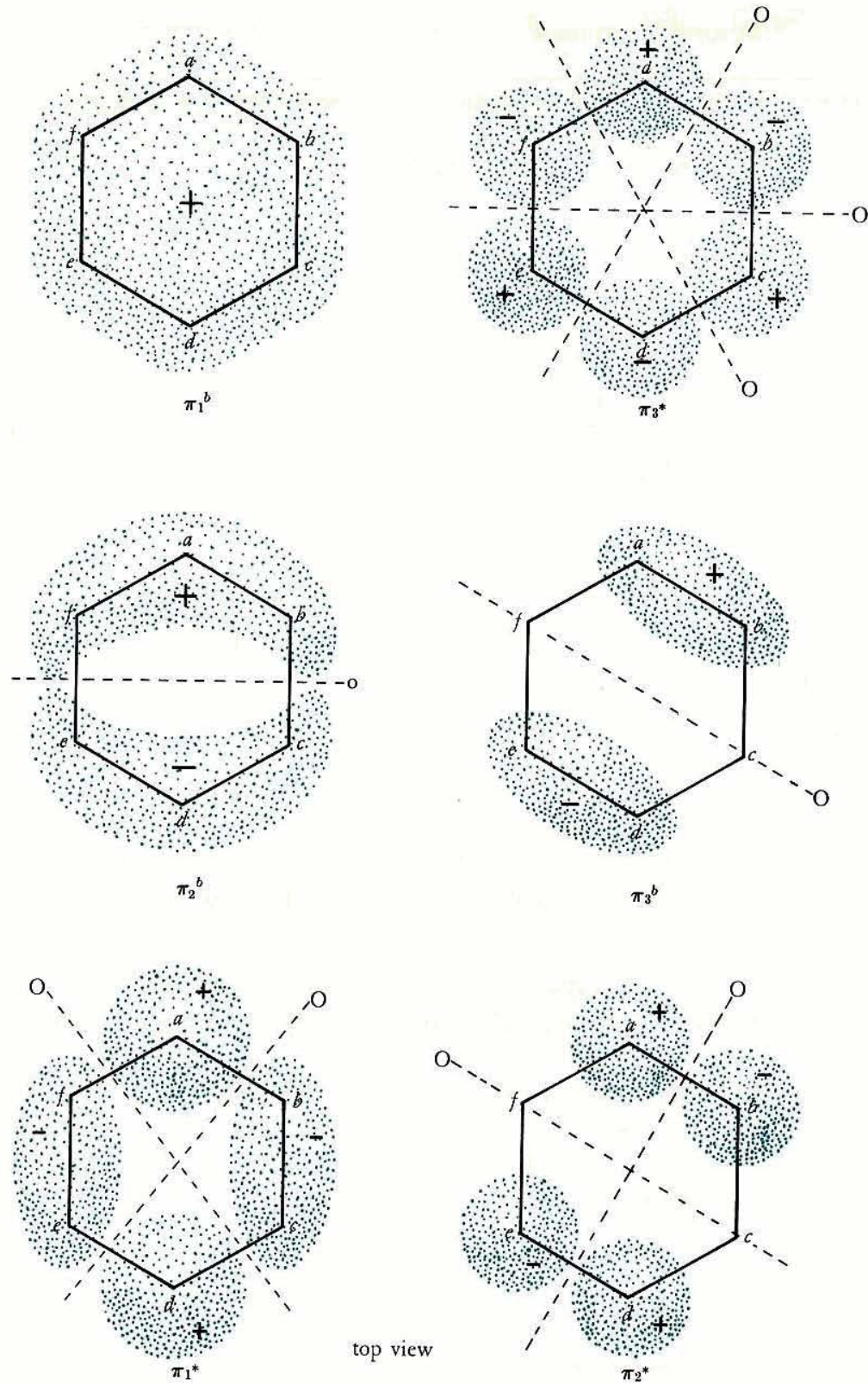

Figure 8-17 Top view of the boundary surfaces of the $\mathrm{C}_{6} \mathrm{H}_{6}$ molecular orbitals. 


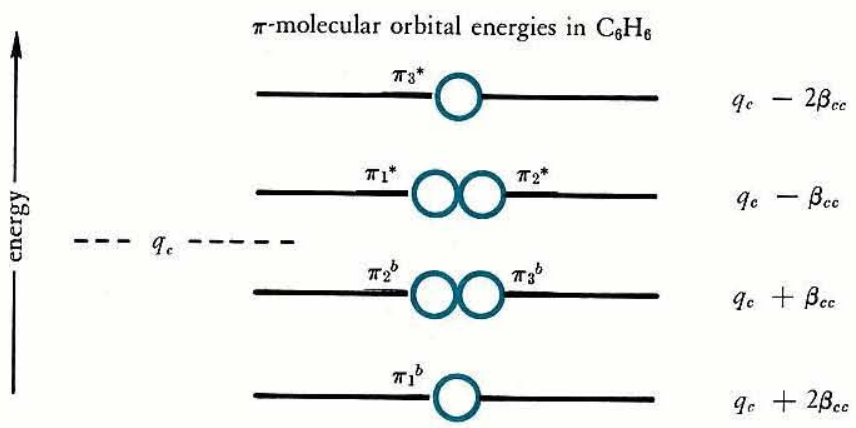

Figure 8-18 Relative energies of the $\pi$ MO's in $\mathbf{C}_{6} \mathbf{H}_{6}$.

\section{PROBLEM}

$8-1$. Show that $\psi\left(\pi_{2}^{b}\right)$ and $\psi\left(\pi_{3}^{b}\right)$ are degenerate in energy, with $E=q_{c}+\beta_{c c}$. Show that $\psi\left(\pi_{1}^{*}\right)$ and $\psi\left(\pi_{2}^{*}\right)$ are degenerate in energy, with $E=q_{c}-\beta_{c c}$. Show that the energy of $\psi\left(\pi_{3}^{*}\right)$ is $q_{c}-2 \beta_{c c}$.

\section{8-16 Ground state of $\mathrm{C}_{6} \mathrm{H}_{6}$}

There are a total of thirty valence electrons in benzene. Twentyfour are used in $\sigma$ bonding ( $\operatorname{six} \mathrm{C}-\mathrm{C}$, six $\mathrm{C}-\mathrm{H}$ bonds), leaving six for the $\pi$-molecular-orbital levels shown in Fig. 8-18. This gives the ground state $\left(\pi_{1}^{b}\right)^{2}\left(\pi_{2}^{b}\right)^{2}\left(\pi_{3}^{b}\right)^{2}$, and a total of three $\pi$ bonds. Each carbon-carbon bond consists of one full $\sigma$ bond and half a $\pi$ bond. The $\mathrm{C}=\mathrm{C}$ bond length in $\mathrm{C}_{6} \mathrm{H}_{6}, 1.397 \mathrm{~A}$, lies between the $\mathrm{C}-\mathrm{C}$ and $\mathrm{C}=\mathrm{C}$ bond lengths.

The common bonding pictures of benzene are shown in Fig. 8-19.

\section{8-17 RESONANCE ENERGY IN $\mathrm{C}_{6} \mathrm{H}_{6}$}

Benzene is actually more stable than might be expected for a system of six $\mathrm{C}-\mathrm{C}$ single bonds and three $\mathrm{C}-\mathrm{C} \pi$ bonds. This added stability is due to the fact that the electrons in the three $\pi$ bonds are delocalized over all six carbons. This is evident both from the molec- 


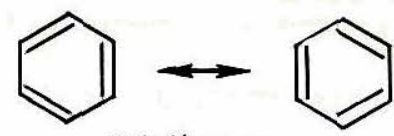

Kekulé structures

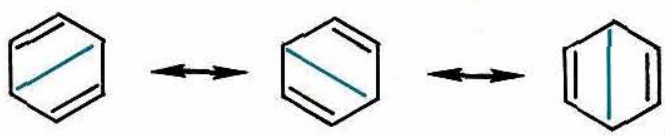

Dewar structures

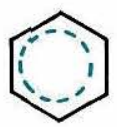

simple MO picture

\section{Figure 8-19 Common representations of the bonding in $\mathrm{C}_{6} \mathrm{H}_{6}$.}

ular orbitals shown in Fig. 8-17 and from the valence-bond structures shown in Fig. 8-19.

In the $\mathrm{MO}$ view, the total gain in $\mathrm{C}_{6} \mathrm{H}_{6}$ stability due to $\pi$ bonding is calculated in units of $\beta_{c c}$ as follows:

$$
\begin{aligned}
\left.2 \text { electrons (in } \pi_{1}^{b}\right) & \times 2 \beta_{c c}=4 \beta_{c c} \\
\left.2 \text { electrons (in } \pi_{2}^{b}\right) & \times \beta_{c c}=2 \beta_{c c} \\
2 \text { electrons (in } \pi_{3}^{b} \text { ) } & \times \beta_{c c}=\frac{2 \beta_{c c}}{8 \beta_{c c}} \\
\text { total } &
\end{aligned}
$$

If we did not allow the delocalization of electrons in $\mathrm{C}_{6} \mathrm{H}_{6}$, we would have a system of three isolated double bonds (only one of the Kekulé structures shown in Fig. 8-19). Let us calculate the $\pi$ bonding stability of three isolated double bonds.

An electron in the $\pi^{b}$ orbital of $\mathrm{C}=\mathrm{C}$ is more stable than an electron in a carbon $2 p$ atomic orbital by one $\beta_{c c}$ unit (see Section $8-3$ ). With six electrons in isolated $\pi^{b}$ orbitals, we have $6 \times \beta_{c c}=6 \beta_{c c}$. The delocalization of three $\pi$ bonds in $\mathrm{C}_{6} \mathrm{H}_{6}$ gives an added stability of $8 \beta_{c c}-6 \beta_{c c}=2 \beta_{c c}$. This is the calculated resonance energy in benzene. 
The so-called experimental resonance energy of benzene is obtained by totaling the bond energies of the $\mathrm{C}-\mathrm{C}, \mathrm{C}=\mathrm{C}$, and $\mathrm{C}-\mathrm{H}$ bonds present and comparing the total with the experimentally known value for the heat of formation of $\mathrm{C}_{6} \mathrm{H}_{6}$. The difference indicates that benzene is about $40 \mathrm{kcal} /$ mole more stable than the sum of the bond energies for a system of six $\mathrm{C}-\mathrm{H}$, three $\mathrm{C}-\mathrm{C}$, and three isolated $\mathrm{C}=\mathrm{C}$ units would suggest.

The value of $\beta_{c c}$ derived from the experimental resonance energy is therefore $-20 \mathrm{kcal} / \mathrm{mole}$. This value differs substantially from the value of $-87 \mathrm{kcal} /$ mole obtained from the absorption spectrum of $\mathrm{C}_{2} \mathrm{H}_{4}$. It is a general result that the resonance-energy $\beta$ 's are much smaller than the spectroscopic $\beta$ 's.

SUPPLEMENTARY PROBLEMS

1. Calculate the energies of the $\pi$ molecular orbitals for $\mathrm{C}_{2} \mathrm{H}_{2}$.

2. Give the "bent-bond" descriptions of $\mathrm{C}_{2} \mathrm{H}_{2}$; of $\mathrm{H}_{2} \mathrm{CO}$; of $\mathrm{HCN}$. 


\section{IX}

\section{Bonds Involving $d$ Valence Orbitals}

\section{9-1 INTRODUCTION}

There are many structures in which the central atom requires one 1 or more $d$ valence orbitals to complete a set of $\sigma$ bonding orbitals. The most important of these structures are square planar, trigonal bipyramidal, square pyramidal, and octahedral; examples are shown in Fig. 9-1. Transition-metal ions have available a very stable set of $d$ valence orbitals. The bonding in complexes formed between transition-metal ions and a large number of molecules and other ions undoubtedly involves $d$ orbitals. In this chapter we shall describe the bonding between metal ions and ligands ${ }^{1}$ in certain representative metal complexes.

\section{9-2 THE OCTAHEDRAL COMPLEX $\mathrm{Ti}\left(\mathrm{H}_{2} \mathrm{O}\right)_{6}{ }^{3+}$}

The $\mathrm{Ti}^{3+}$ ion forms a stable complex ion with six water molecules. The structure around the $\mathrm{Ti}^{3+}$ ion is octahedral, as shown in Fig. 9-2.

1 Groups attached to metal ions in complexes are called ligands.

176 
Bonds Involving d Valence Orbitals
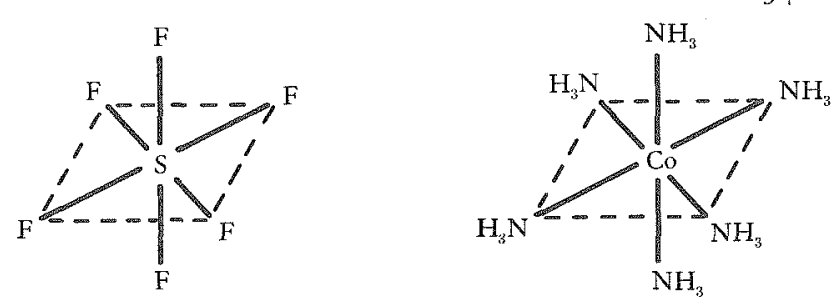

octahedral

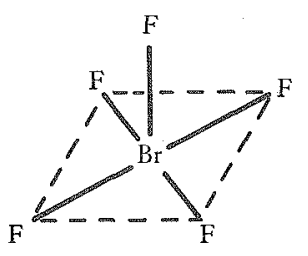

square pyramidal

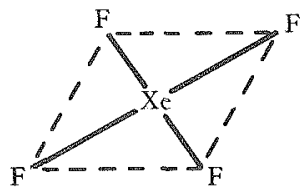

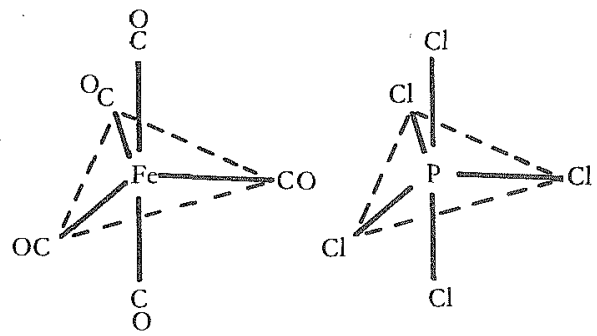

trigonal bipyramidal

square planar

Tigure 9-1 Examples of structures in which orbitals are used in bonding. 


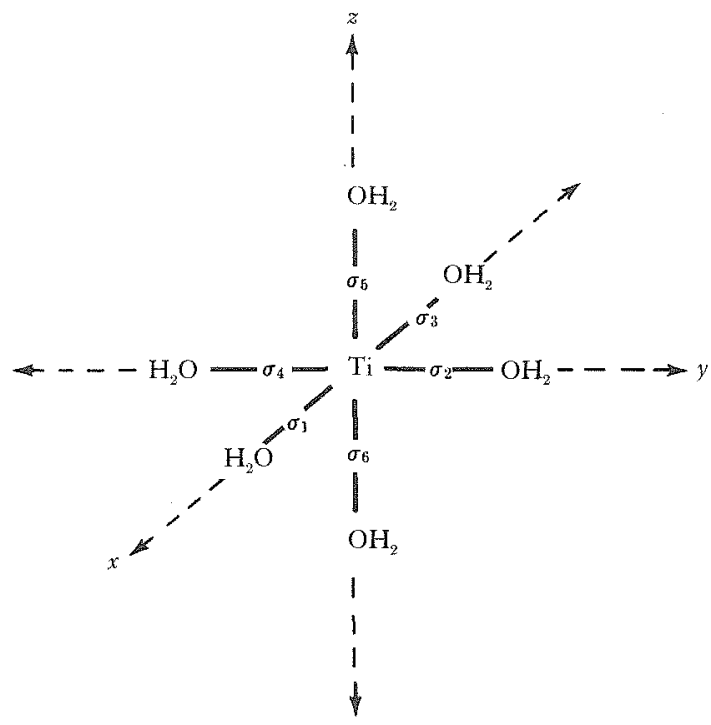

Figure 9-2 Coordinate system for $\mathrm{Ti}\left(\mathrm{H}_{2} \mathrm{O}\right)_{6}{ }^{3+}$.

The titanium has five $3 d$, one $4 s$, and three $4 p$ valence orbitals that can be used in constructing molecular orbitals. Each water molecule must furnish one $\sigma$ valence orbital, which, in accord with the discussion in Chapter VIII, is approximately an $s p^{3}$ hybrid orbital. We shall not specify the exact $s$ and $p$ character of the water $\sigma$ valence orbital, however, but simply refer to it as $\sigma$.

The metal orbitals that can form $\sigma$ molecular orbitals are $3 d_{x^{2}-y^{2}}$, $3 d_{z^{2}}, 4 s, 4 p_{x}, 4 p_{y}$, and $4 p_{z}$. Since the sign of the $4 s$ orbital does not change over the boundary surface, the proper linear combination of ligand orbitals for $4 s$ is

$$
\sigma_{1}+\sigma_{2}+\sigma_{3}+\sigma_{4}+\sigma_{5}+\sigma_{6}
$$

This is shown in Fig. 9-3. The wave function for the molecular orbital involving the metal $4 s$ orbital is therefore

$$
\psi\left(\sigma_{s}\right)=c_{1} 4 s+c_{2}\left(\sigma_{1}+\sigma_{2}+\sigma_{3}+\sigma_{4}+\sigma_{5}+\sigma_{6}\right)
$$

We find the other molecular orbitals by matching the metal-orbital 


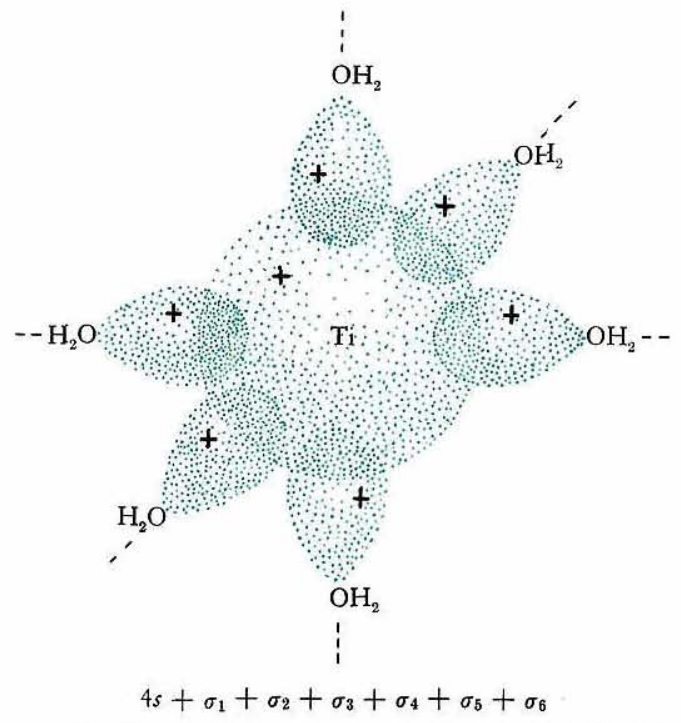

Figure 9-3 Overlap of the titanium 4s orbital with the $\sigma$ orbitals of the water molecules.

lobes with ligand $\sigma$ orbitals that have the proper sign and magnitude. This procedure is shown in Fig. 9-4. The wave functions are:

$$
\begin{aligned}
\psi\left(\sigma_{x}\right) & =c_{3} 4 p_{x} \quad+c_{4}\left(\sigma_{1}-\sigma_{3}\right) \\
\psi\left(\sigma_{y}\right) & =c_{3} 4 p_{y} \quad+c_{4}\left(\sigma_{2}-\sigma_{4}\right) \\
\psi\left(\sigma_{z}\right) & =c_{3} 4 p_{z} \quad+c_{4}\left(\sigma_{5}-\sigma_{6}\right) \\
\psi\left(\sigma_{x^{2}-y^{2}}\right) & =c_{5} 3 d_{x^{2}-y^{2}}+c_{6}\left(\sigma_{1}-\sigma_{2}+\sigma_{3}-\sigma_{4}\right) \\
\psi\left(\sigma_{z^{2}}\right) & =c_{7} 3 d_{z^{2}} \quad+c_{8}\left(2 \sigma_{5}+2 \sigma_{6}-\sigma_{1}-\sigma_{2}-\sigma_{3}-\sigma_{4}\right)
\end{aligned}
$$

\section{9-3 ENERGY LEVELS IN $\mathrm{Ti}\left(\mathrm{H}_{2} \mathrm{O}\right)_{6}{ }^{3+}$}

Figure 9-4 shows $4 p_{x}, 4 p_{y}$, and $4 p_{z}$ to be equivalent in an octahedral complex; on this basis the $\sigma_{x}, \sigma_{y}$, and $\sigma_{z}$ molecular orbitals are degenerate in energy. Although it is not obvious from Fig. 9-4, the 


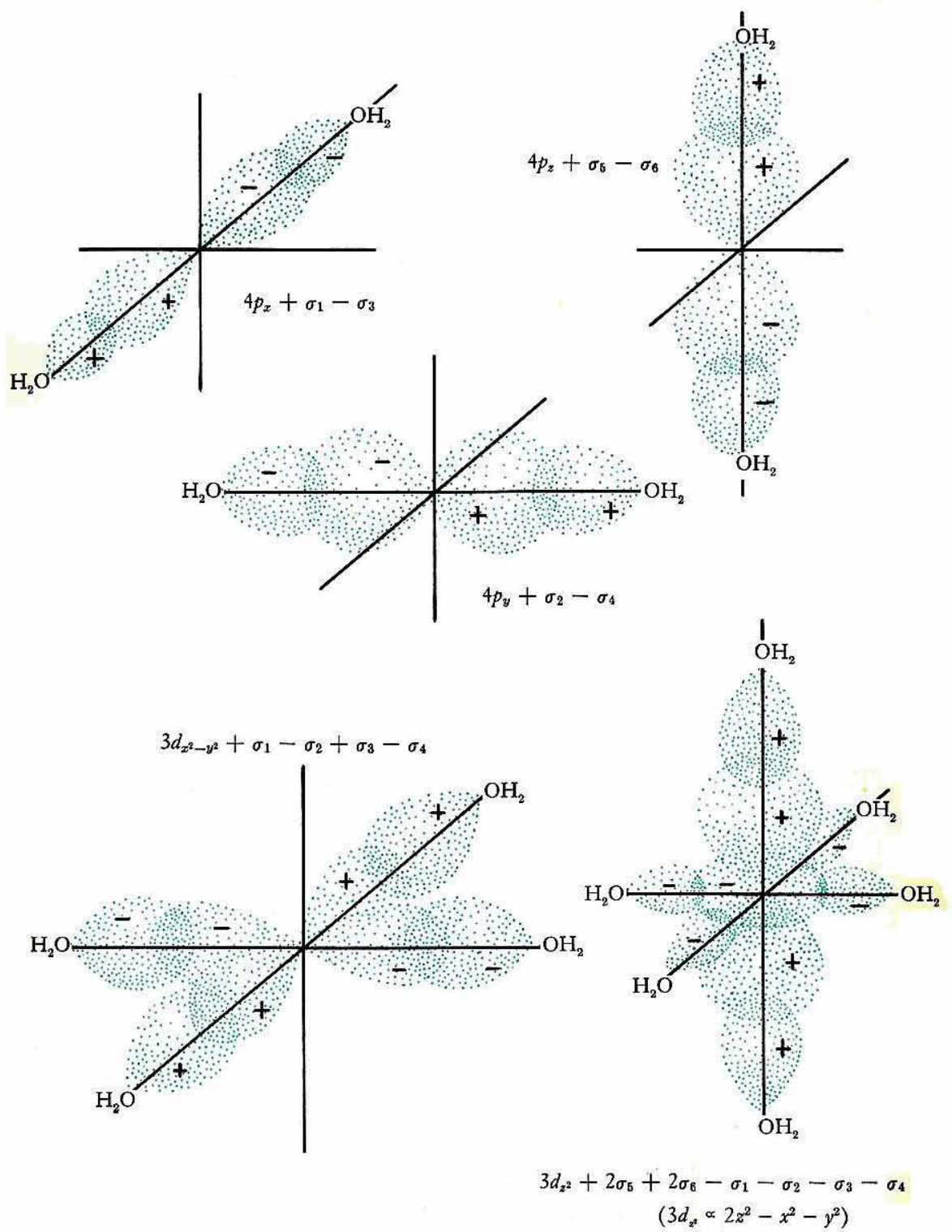

Figure 9-4 Overlap of the titanium $3 d$ and $4 p \sigma$ orbitals with the $\sigma$ orbitals of the water molecules. 
$3 d_{x^{2}-y^{2}}$ and $3 d_{z^{2}}$ orbitals are also equivalent in an octahedral complex, and $\sigma_{x^{2}-y^{2}}$ and $\sigma_{z^{2}}$ are degenerate in energy. We shall solve a problem at the end of this chapter to prove the equivalence of $3 d_{x^{2}-y^{2}}$ and $3 d_{z} z^{2}$. Finally, we see that, including the $\sigma_{s}$ orbital, there are three sets of $\sigma$ molecular orbitals in an octahedral complex: $\sigma_{s} ; \sigma_{x}, \sigma_{y}, \sigma_{z}$;

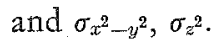

We have used all but three of the metal valence orbitals in the $\sigma$ molecular orbitals. We are left with $3 d_{x z}, 3 d_{y z}$, and $3 d_{x y}$. These orbitals are situated properly for $\pi$ bonding in an octahedral complex, as will be discussed later. However, since water is not a good $\pi$ bonding ligand, we shall consider that the $3 d_{x z}, 3 d_{y z}$, and $3 d_{x y}$ orbitals are essentially nonbonding in $\operatorname{Ti}\left(\mathrm{H}_{2} \mathrm{O}\right)_{6}{ }^{3+}$. The three $d_{\pi}$ orbitals are clearly equivalent in an octahedral complex, and we have the degenerate set: $\pi_{x z}, \pi_{y z}, \pi_{x y}$.

In order to construct an energy-level diagram for $\mathrm{Ti}\left(\mathrm{H}_{2} \mathrm{O}\right)_{6}{ }^{3+}$, we must know something about the relative energies of the starting orbitals $3 d, 4 s, 4 p$, and $\sigma_{\mathrm{H}_{2} \mathrm{O}}$. In this case, $\sigma_{\mathrm{H}_{2} \mathrm{O}}$ is more stable than any of the metal valence orbitals. This is fairly general in metal complexes, and in energy-level diagrams the ligand $\sigma$ valence orbitals are shown to be more stable than the corresponding metal valence orbitals. It is also generally true that the order of increasing energy for the metal valence orbitals in transition-metal complexes is $n d<(n+1) s<(n+1) p$.

The energy-level diagram for $\mathrm{Ti}\left(\mathrm{H}_{2} \mathrm{O}\right)_{6}{ }^{3+}$ is shown in Fig. 9-5. There are three sets of bonding orbitals and three sets of antibonding orbitals. The virtually nonbonding $\pi(d)$ orbitals are less stable than the bonding $\sigma(d)$ set but more stable than the antibonding $\sigma(d)$ set. The relative energies of the three bonding $\sigma$ sets are not known. The order given in Fig. 9-5 was obtained from a calculation that is beyond the level of our discussion.

\section{9-4 Ground state of $\mathrm{Ti}\left(\mathrm{H}_{2} \mathrm{O}\right)_{6}{ }^{3+}$}

We must count every electron in the valence orbitals used to construct the diagram in Fig. 9-5. The complex is considered to be composed of $\mathrm{Ti}^{3+}$ and six water molecules. Each of the six $\sigma$ valence orbitals of the water molecules furnishes two electrons, for a total of 


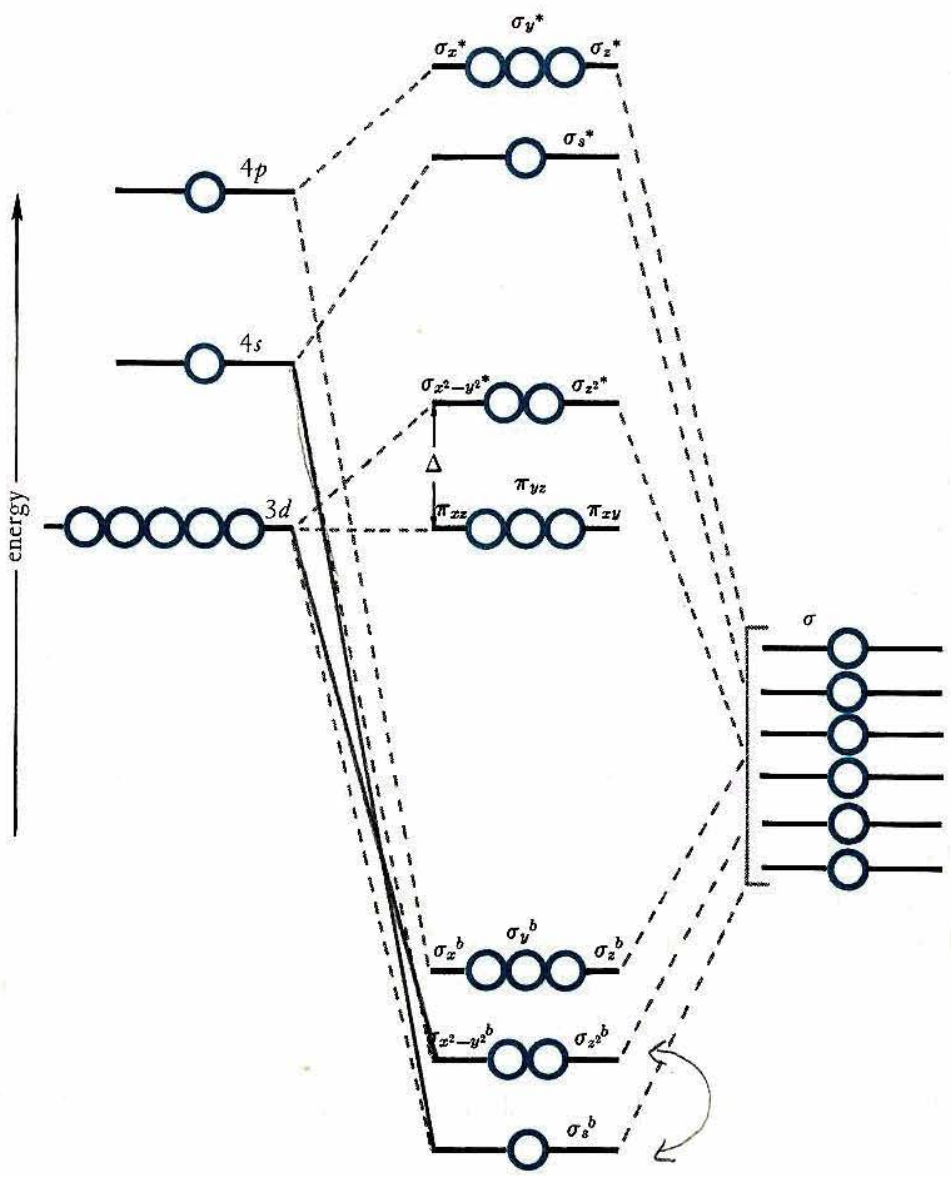

Figure 9-5 Relative orbital energies in $\mathrm{Ti}\left(\mathrm{H}_{2} \mathrm{O}\right)_{6}{ }^{3+}$.

twelve. Since the electronic structure of $\mathrm{Ti}^{3+}$ is $(3 d)^{1}$, we have a total of thirteen electrons to place in the molecular orbitals shown in Fig. 9-5. The ground state of $\mathrm{Ti}\left(\mathrm{H}_{2} \mathrm{O}\right)_{6}{ }^{3+}$ is therefore

$$
\left(\sigma_{s}^{b}\right)^{2}\left(\sigma_{x, y, z}^{b}\right)^{6}\left(\sigma^{b} x^{2}-y^{2}, z^{2}\right)^{4}\left(\pi_{x z, y z, x y}\right)^{1} \quad S=\frac{1}{2}
$$


There is one unpaired electron in the $\pi(d)$ level. Consistent with this ground state, $\mathrm{Ti}\left(\mathrm{H}_{2} \mathrm{O}\right)_{6}{ }^{3+}$ is paramagnetic, with $S=\frac{1}{2}$.

The electrons in $\sigma$ bonding orbitals are mainly localized on the water molecules, since the $\sigma$ valence orbital of $\mathrm{H}_{2} \mathrm{O}$ is more stable than the metal orbitals. The nonbonding and antibonding orbitals, on the other hand, are mainly located on the metal. We shall focus our attention in the sections to follow on the molecular orbitals that are mainly based on the metal and derived from the $3 d$ valence orbitals.

\section{9-5 THE ELECTRONIC SPECTRUM OF $\mathrm{Ti}\left(\mathrm{H}_{2} \mathrm{O}\right)_{6}{ }^{3+}$}

The difference in energy between $\sigma^{*}(d)$ and $\pi(d)$ is called $\Delta$ or $10 D q$. Excitation of the electron in $\pi(d)$ to $\sigma^{*}(d)$ occurs with absorption of light in the visible region of the spectrum, and $\mathrm{Ti}\left(\mathrm{H}_{2} \mathrm{O}\right)_{6}{ }^{3+}$ is therefore colored reddish-violet. The electronic spectrum of $\mathrm{Ti}\left(\mathrm{H}_{2} \mathrm{O}\right)_{6}{ }^{3+}$ is shown in Fig. 9-6. The maximum absorption occurs at $4930 \mathrm{~A}$, or $20,300 \mathrm{~cm}^{-1}$. The value of the splitting $\Delta$ is usually expressed in $\mathrm{cm}^{-1}$ units; thus we say that $\mathrm{Ti}\left(\mathrm{H}_{2} \mathrm{O}\right)_{6}{ }^{3+}$ has a $\Delta$ of 20,300 $\mathrm{cm}^{-1}$.

The colors of many other transition-metal complexes are also due to such " $d-d$ " transitions.
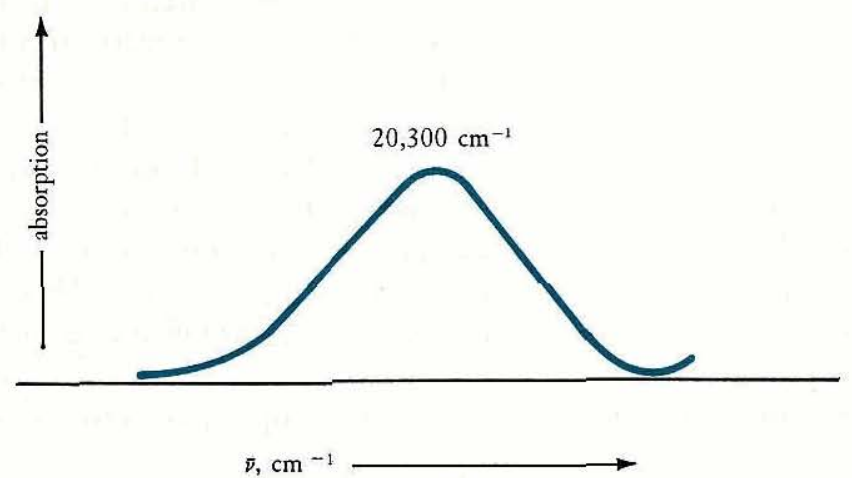

Figure 9-6 The absorption spectrum of $\mathrm{Ti}\left(\mathrm{H}_{2} \mathrm{O}\right)_{6}{ }^{3+}$ in the visible region. 


\section{9-6 VALENCE-BOND THEORY FOR $\mathrm{Ti}\left(\mathrm{H}_{2} \mathrm{O}\right)_{6}{ }^{3+}$}

The localized bonding scheme for $\mathrm{Ti}\left(\mathrm{H}_{2} \mathrm{O}\right)_{6}^{3+}$ is obtained by first constructing six equivalent hybrid orbitals that are octahedrally directed. We use the six $\sigma$ valence orbitals of Ti for this purpose: $3 d_{x^{2}-y^{2}}, 3 d_{z^{2}}, 4 s, 4 p_{x}, 4 p_{y}$, and $4 p_{z}$. Thus we want to construct six $d^{2} s p^{3}$ hybrid orbitals, each with one-third $d$ character, one-sixth $s$ character, and one-half $p$ character.

Referring back to Fig. 9-2, let us form linear combinations of the $d, s$, and $p$ valence orbitals that direct large lobes at the six ligands. We first construct the orbitals that are directed toward ligands (5) and (6). We shall call these orbitals $\psi_{5}$ and $\psi_{6}$, respectively. The metal orbitals that can $\sigma$ bond with (5) and (6) are $3 d_{z}^{2}$, $4 s$, and $4 p_{z}$. Choosing the coefficients of the $3 d_{z}^{2}, 4 s$, and $4 p_{z}$ orbitals so that $\psi_{5}$ and $\psi_{6}$ have the desired $d, s$, and $p$ character, we obtain the following hybrid-orbital wave functions:

$$
\begin{aligned}
& \psi_{5}=\frac{1}{\sqrt{3}} 3 d_{z^{2}}+\frac{1}{\sqrt{6}} 4 s+\frac{1}{\sqrt{2}} 4 p_{z} \\
& \psi_{6}=\frac{1}{\sqrt{3}} 3 d_{z}{ }^{2}+\frac{1}{\sqrt{6}} 4 s-\frac{1}{\sqrt{2}} 4 p_{z}
\end{aligned}
$$

The positive coefficient of $4 p_{z}$ in $\psi_{5}$ directs a large lobe toward (5), and the negative coefficient of $4 p_{2}$ in $\psi_{6}$ directs a large lobe toward (6).

The orbitals directed toward (1) and (3) are constructed from the $3 d_{x^{2}}-y^{2}, 3 d_{z^{2}}, 4 s$ and $4 p_{x}$ metal orbitals. The orbitals directed toward (2) and (4) are constructed from the $3 d_{x^{2}-y^{2}}, 3 d_{z} z^{2}, 4 s$, and $4 p_{y}$ orbitals. The coefficients of $4 s$ and $4 p$ pose no problem, but we have to divide the one-third $d$ character in each hybrid orbital between $3 d_{2}{ }^{2}$ and $3 d x^{2}-y^{2}$. We see from Eqs. (9-8) and (9-9) that we have "used up" two-thirds of the $3 d_{z}^{2}$ orbital in $\psi_{5}$ and $\psi_{6}$. Thus we must divide the remaining one-third equally among $\psi_{1}, \psi_{2}, \psi_{3}$, and $\psi_{4}$. This means that each of $\psi_{1}, \psi_{2}, \psi_{3}$, and $\psi_{4}$ has one-twelfth $3 d_{2}{ }^{2}$ character and onefourth $3 d x^{2}-y^{2}$ character. Choosing the signs of the coefficients so that a large lobe is directed towatd each ligand in turn, we have:

$$
\psi_{1}=\frac{1}{2} 3 d x^{2}-y^{2}-\frac{1}{\sqrt{12}} 3 d z^{2}+\frac{1}{\sqrt{6}} 4 s+\frac{1}{\sqrt{2}} 4 p_{x}
$$




$$
\begin{aligned}
& \psi_{2}=-\frac{1}{2} 3 d_{x^{2}-y^{2}}-\frac{1}{\sqrt{12}} 3 d_{z^{2}}+\frac{1}{\sqrt{6}} 4 s+\frac{1}{\sqrt{2}} 4 p_{y} \\
& \psi_{3}=\frac{1}{2} 3 d_{x^{2}-y^{2}}-\frac{1}{\sqrt{12}} 3 d_{z^{2}}+\frac{1}{\sqrt{6}} 4 s-\frac{1}{\sqrt{2}} 4 p_{x} \\
& \psi_{4}=-\frac{1}{2} 3 d_{x^{2}-y^{2}}-\frac{1}{\sqrt{12}} 3 d_{z^{2}}+\frac{1}{\sqrt{6}} 4 s-\frac{1}{\sqrt{2}} 4 p_{y}
\end{aligned}
$$

These six localized $d^{2} s p^{3}$ orbitals are used to form electron-pair bonds with the six water molecules. The valence-bond description of the ground state of $\mathrm{Ti}\left(\mathrm{H}_{2} \mathrm{O}\right)_{6}{ }^{3+}$ is shown in Fig. 9-7. The unpaired elec-
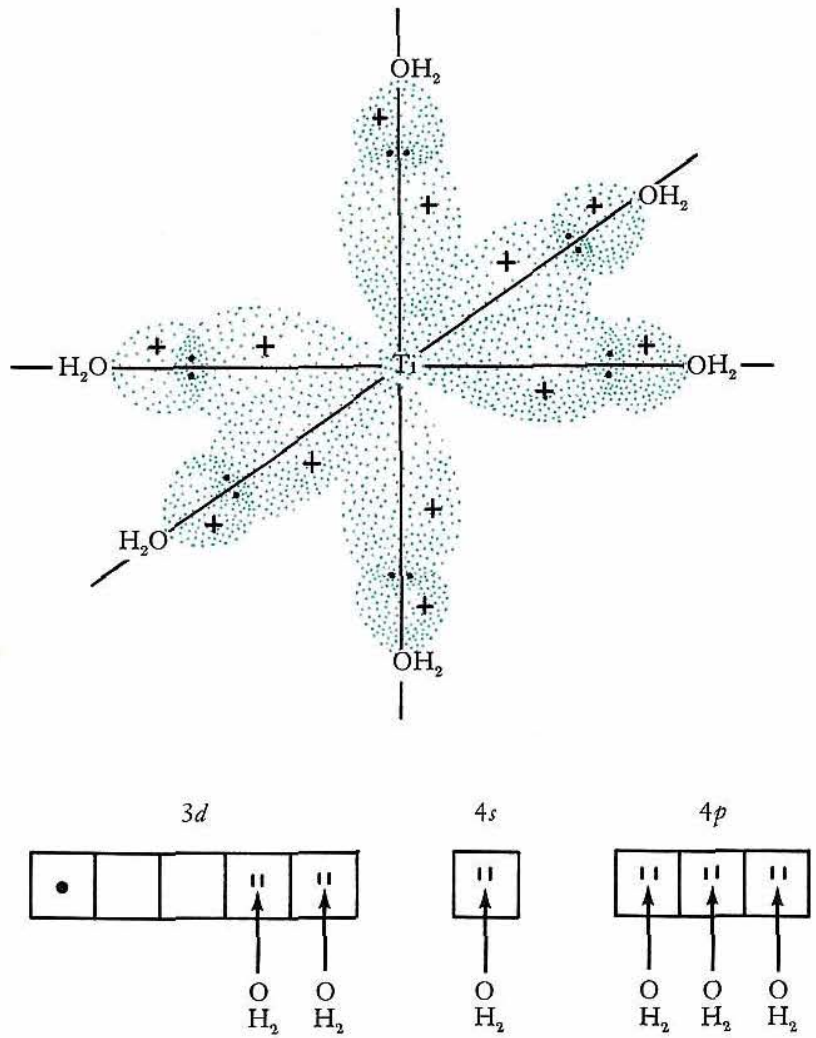

Figure 9-7 Valence-bond representations of $\mathrm{Ti}\left(\mathrm{H}_{2} \mathrm{O}\right)_{6}{ }^{3+}$. 
tron is placed in one of the $d$ orbitals that has not been used to construct hybrid bond orbitals. This simple valence-bond orbital diagram is also shown in Fig. 9-7.

\section{9-7 CRYSTAL-FIELD THEORY FOR $\mathrm{Ti}\left(\mathrm{H}_{2} \mathrm{O}\right)_{6}{ }^{3+}$}

In the crystal-field-theory formulation of a metal complex, we consider the ligands as point charges or point dipoles. The crystalfield model is shown in Fig. 9-8. The point charges or point dipoles constitute an electrostatic field, which has the symmetry of the complex. The effect of this electrostatic field on the energies of the metal $d$ orbitals is the subject of our interest.

Let us examine the energy changes in the $3 d$ orbitals of $\mathrm{Ti}^{3+}$ that result from placement in an octahedral field of point dipoles (the water molecules). First, all the $d$ orbital energies are raised, owing to the proximity of the negative charges. More important, however, the two orbitals $\left(3 d_{z^{2}}, 3 d_{x^{2}-y^{2}}\right)$ that point directly at the negative charges are raised higher in energy than the three orbitals $\left(3 d_{x z}\right.$,

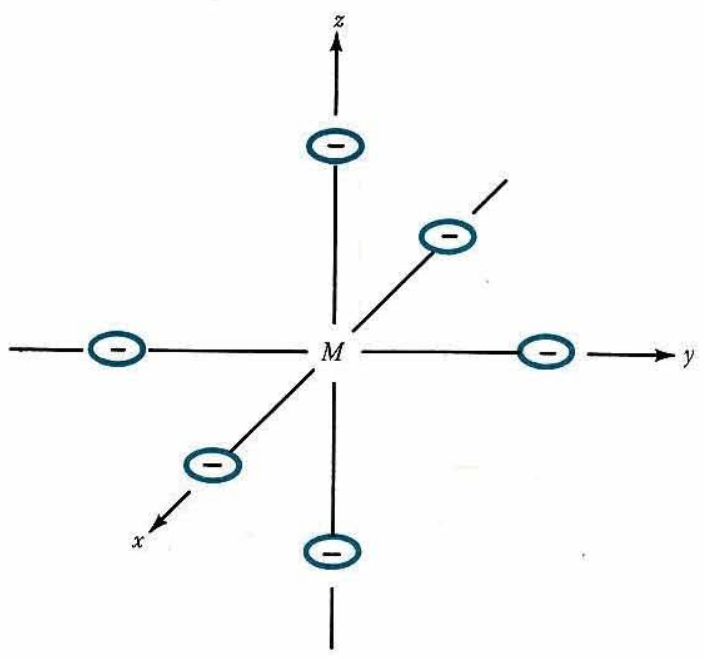

Figure 9-8 An octahedral field of point charges. 


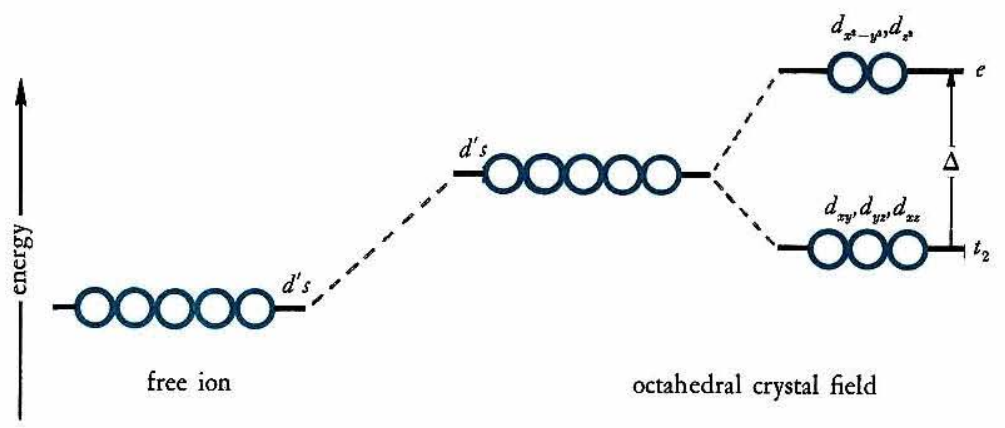

Figure 9-9 Splitting of the metal $d$ orbitals in an octahedral crystal field.

$\left.3 d_{y z}, 3 d_{x y}\right)$ that are directed at points between the negative charges. Thus we have a splitting of the five $d$ orbitals in an octahedral crystal field as shown in Fig. 9-9. It is convenient to use the group-theoretical symbols for the split $d$ levels. The $3 d_{z}{ }^{2}$ and $3 d_{x^{2}-y^{2}}$ orbitals form the degenerate set called $e$, and the $3 d_{x z}, 3 d_{y z}$, and $3 d_{x y}$ orbitals form the degenerate set called $t_{2}$. The separation of $e$ and $t_{2}$ is again designated $\Delta$ or $10 D q$.

The one $d$ electron in $\mathrm{Ti}^{3+}$ is placed in the more stable $t_{2}$ orbitals in the ground state. The excitation of this electron from $t_{2}$ to $e$ is responsible for the spectral band shown in Fig. 9-6.

9-8 RELATIONSHIP OF THE GENERAL MOLECULAR-ORBITAL TREATMENT TO THE VALENCE-BOND AND CRYSTAL-FIELD THEORIES

The valence-bond and crystal-field theories describe different parts of the general molecular-orbital diagram shown in Fig. 9-5. The $\sigma$ bonding molecular orbitals are related to the six $d^{2} s p^{3}$ bonding orbitals of the valence-bond theory. The valence-bond theory does not include the antibonding orbitals, and therefore does not provide an explanation for the spectral bands of metal complexes. The $t_{2}$ and $e$ levels of the crystal-field theory are related to the $\pi(d)$ and $\sigma^{*}(d)$ molecular orbitals. A diagram showing the relationship between the three theories is given in Fig. 9-10. 
crystal-field splitting related to splitting between $\sigma^{*}(d)$ and $\pi(d)$ molecular orbitals
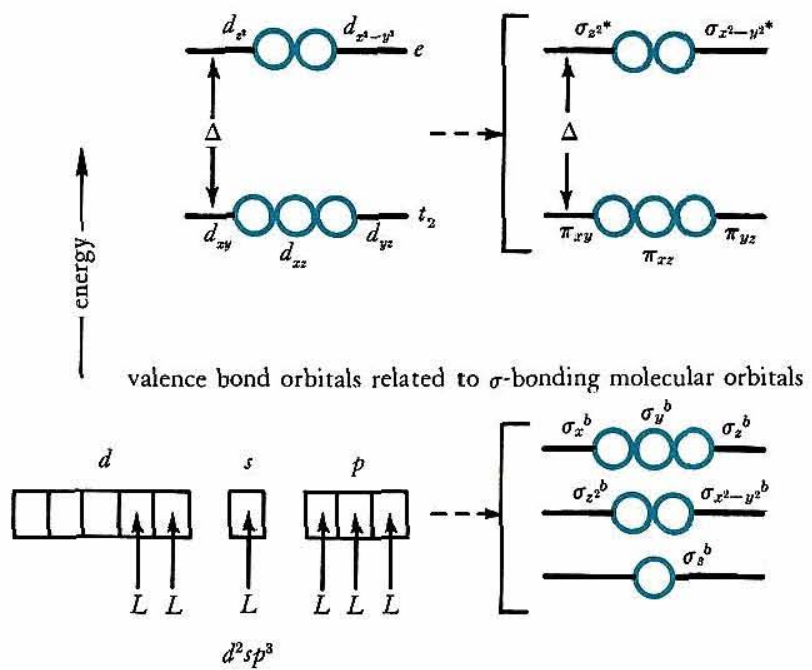

Figure 9-10 Comparison of the three theories used to describe the electronic structures of transition-metal complexes.

\section{9-9 TYPES OF $\pi$ BONDING IN METAL COMPLEXES}

The $d_{x z}, d_{y z}$, and $d_{x y}$ orbitals may be used for $\pi$ bonding in octahedral complexes. Consider a complex containing six chloride ligands. Each of the $d_{\pi}$ orbitals overlaps with four ligand $\pi$ orbitals, as shown in Fig. 9-11. In the bonding orbital, some electronic charge from the chloride is transferred to the metal. We call this ligand-to-metal $(L \rightarrow M) \pi$ bonding. The $\pi$ orbitals based on the metal are destabilized in the process and are made antibonding.

If the complex contains a diatomic ligand such as $\mathrm{CN}^{-}$, two types of $\pi$ bonding are possible. Recall from Chapter II that $\mathrm{CN}^{-}$has filled $\pi^{b}$ and empty $\pi^{*}$ molecular orbitals, as shown in Fig. 9-12. The occupied $\pi^{b}$ orbitals can enter into $L \rightarrow M \pi$ bonding with the $3 d_{x z}, 3 d_{y z}$, and $3 d_{x y}$ orbitals. In addition, however, electrons in the metal $\pi(d)$ level can be delocalized into the available $\pi^{*}\left(\mathrm{CN}^{-}\right)$ orbitals, thus preventing the accumulation of too much negative 


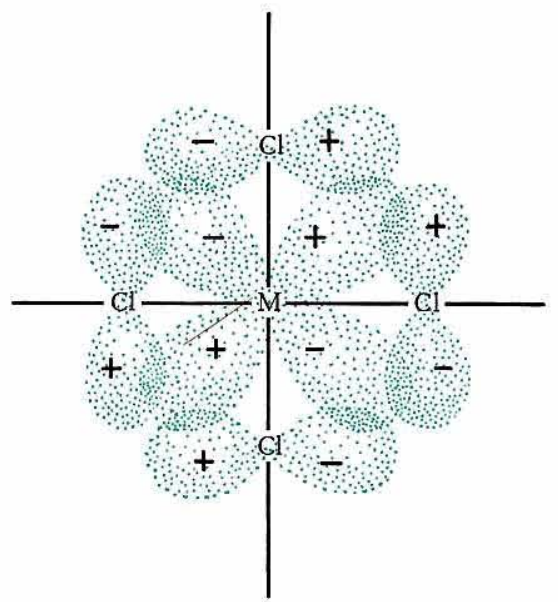

Figure 9-11 Overlap of a $d_{\pi}$ orbital with four ligand $\pi$ orbitals in an octahedral complex.

charge on the metal. This type of bonding removes electronic density from the metal and is called metal-to-ligand $(M \rightarrow L) \pi$ bonding. It is also commonly called back donation or back bonding. Back donation stabilizes the $\pi(d)$ level and makes it less antibonding. Both types of $\pi$ bonding between a $d_{\pi}$ orbital and $\mathrm{CN}^{-}$are shown in Fig. 9-12.

\section{9-10 SQUARE-PLANAR COMPLEXES}

A simple square-planar complex is $\mathrm{PtCl}_{4}{ }^{2-}$. The coordinate system that we shall use to discuss the bonding in $\mathrm{PtCl}_{4}{ }^{2-}$ is pictured in Fig. 9-13.

The metal valence orbitals suitable for $\sigma$ molecular orbitals are $5 d_{x^{2}-y^{2}}, 5 d_{z^{2}}, 6 s, 6 p_{x}$, and $6 p_{y}$. Of the two $d \sigma$ valence orbitals, it is clear that $5 d_{x^{2}-y^{2}}$ interacts strongly with the four ligand $\sigma$ valence orbitals and that $5 d_{z^{2}}$ interacts weakly (most of the $5 d_{z^{2}}$ orbital is directed along the $z$ axis).

The $5 d_{x z}, 5 d_{y z}$, and $5 d_{x y}$ orbitals are involved in $\pi$ bonding with the ligands. The $5 d_{x y}$ orbital interacts with $\pi$ valence orbitals on all four 

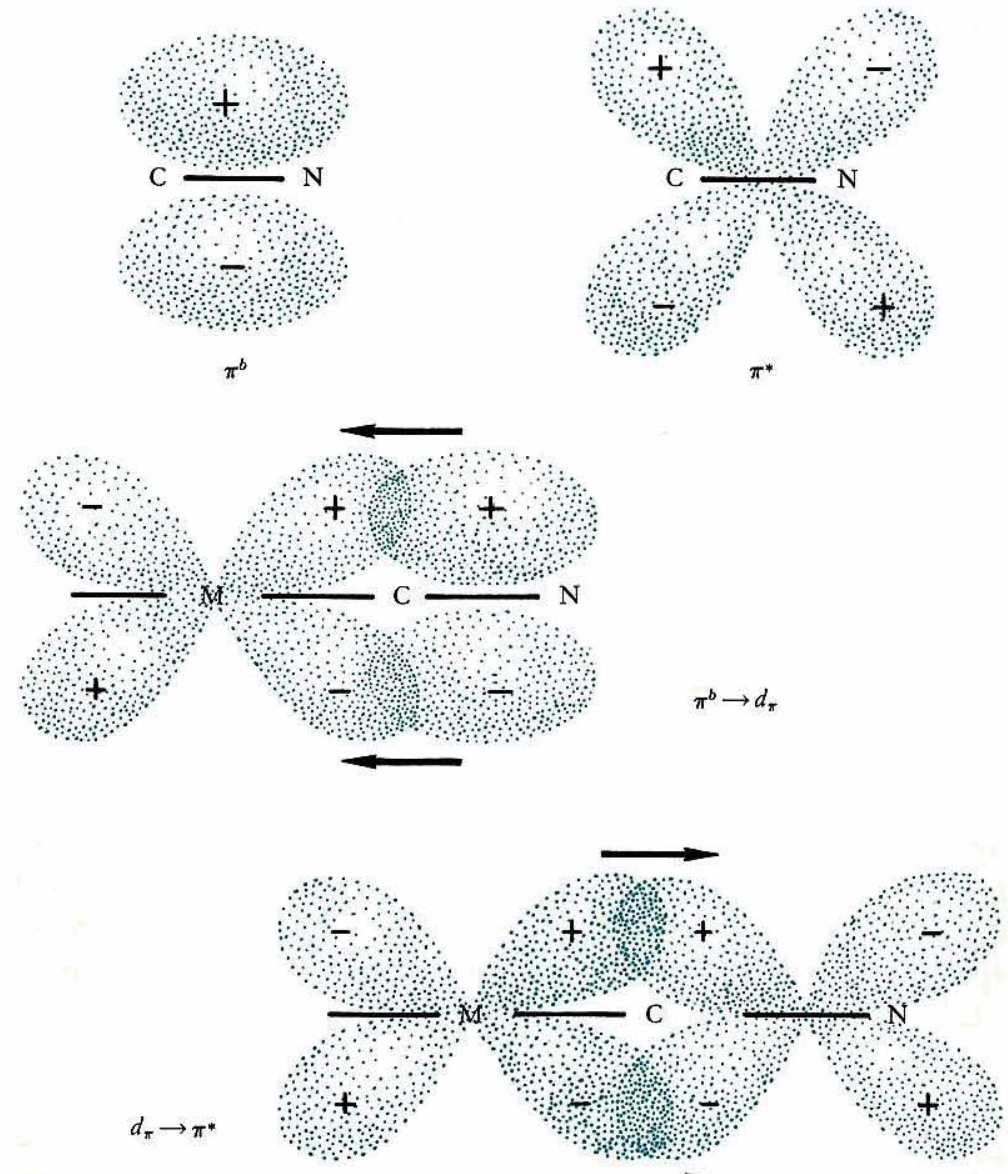

Figure 9-12 Types of $\pi$ bonding between $\mathrm{CN}^{-}$and a metal $\boldsymbol{d}_{\pi}$ orbital.

ligands, whereas $5 d_{x z}$ and $5 d_{y z}$ are equivalent and interact with only two ligands. The overlap of the metal $5 d$ orbitals with the valence orbitals of the four ligands is shown in Fig. 9-14.

We can now construct an approximate energy-level diagram for $\mathrm{PtCl}_{4}{ }^{2-}$. We shall not attempt to pinpoint all the levels, but instead 


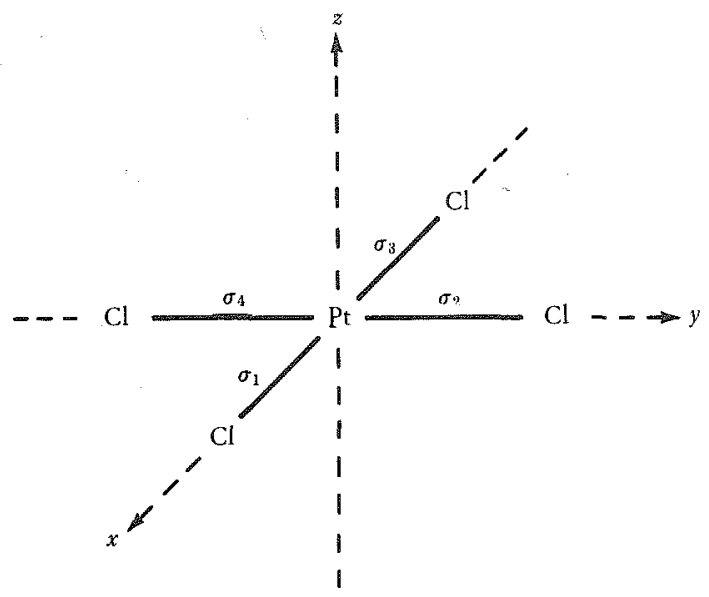

Figure 9-13 Coordinate system for $\mathrm{PtCl}_{4}{ }^{2-}$.

to recognize a few important regions of energy. A simplified energylevel diagram for $\mathrm{PtCl}_{4}{ }^{2-}$ is shown in Fig. 9-15. The most stable orbitals are $\sigma$ bonding and are located on the chlorides. Next in order of stability are the $\pi$ molecular orbitals, also mainly based on the four chlorides. The molecular orbitals derived from the $5 d$ valence orbitals are in the middle of the diagram. They are the antibonding partners of the $\sigma$ and $\pi$ bonding orbitals just described.

We can confidently place the strongly antibonding $\sigma^{*} x^{2}-y^{2}$ highest. We can also place $\pi_{x y}{ }^{*}$ above $\pi^{*} x z, y z$, since $3 d_{x y}$ interacts with all four ligands (see Fig. 9-14). The weakly antibonding $\sigma_{z^{2}}{ }^{*}$ is believed to lie between $\pi_{x y}{ }^{*}$ and $\pi_{x z, y z}^{*}$. However, regardless of the placement of $\sigma_{z^{2}}{ }^{*}$, the most important characteristic of the energy levels in a square-planar complex is that one $d$ level has very high energy whereas the other four are much more stable and bunched together.

Since $\mathrm{Pt}^{2+}$ is $5 d^{8}$ and since the four chlorides furnish eight $\sigma$ and sixteen $\pi$ electrons, the ground state of $\mathrm{PtCl}_{4}{ }^{2-}$ is

$$
\left(\sigma^{b}\right)^{8}(\pi)^{16}\left(\pi^{*} x_{z, y z}\right)^{4}\left(\sigma_{z}^{*}\right)^{2}\left(\pi_{x y}\right)^{2} \quad S=0
$$

The complex is diamagnetic since the eight metal valence electrons 


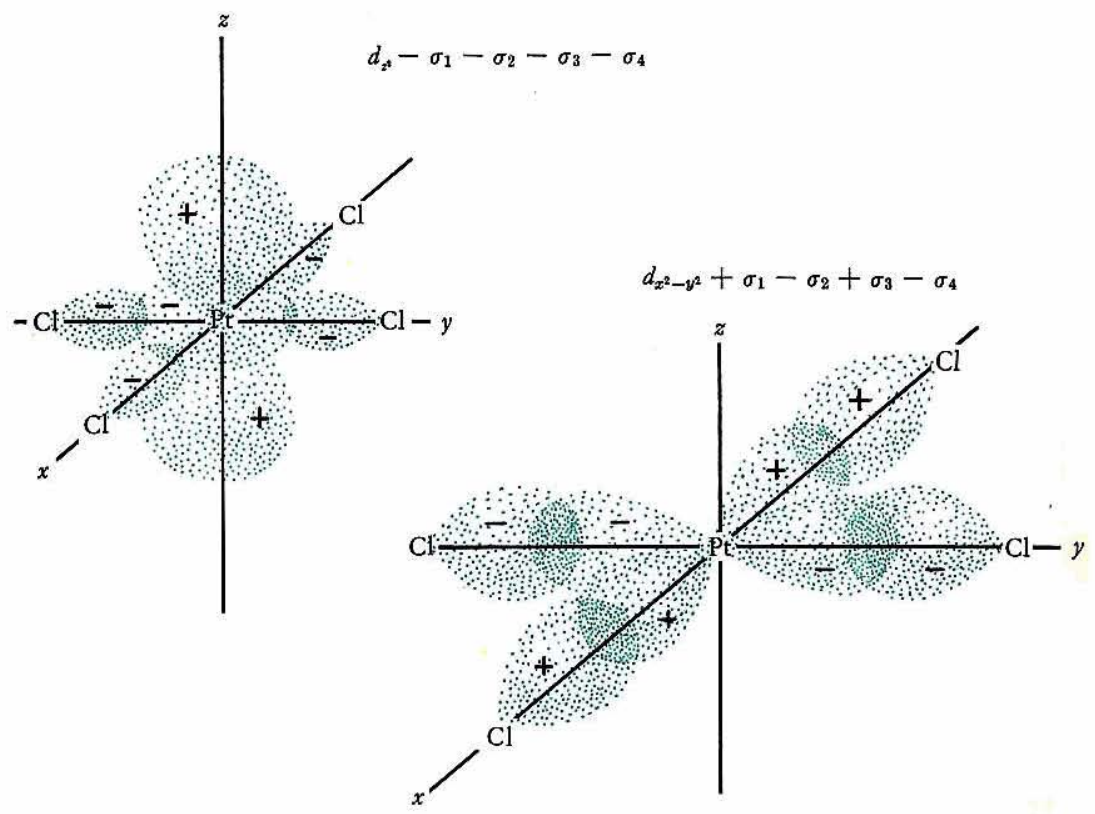

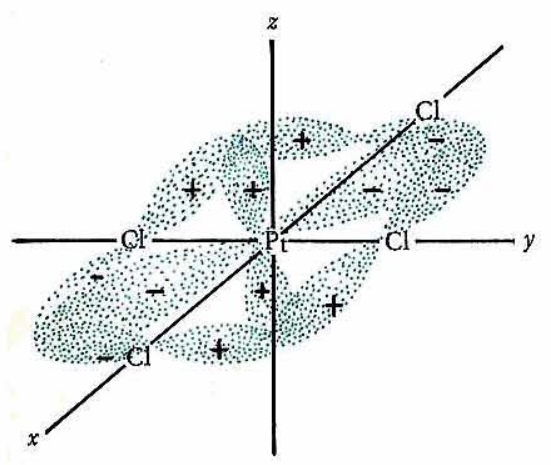

$d_{x y}$ (4 ligand $\pi$ orbitals)

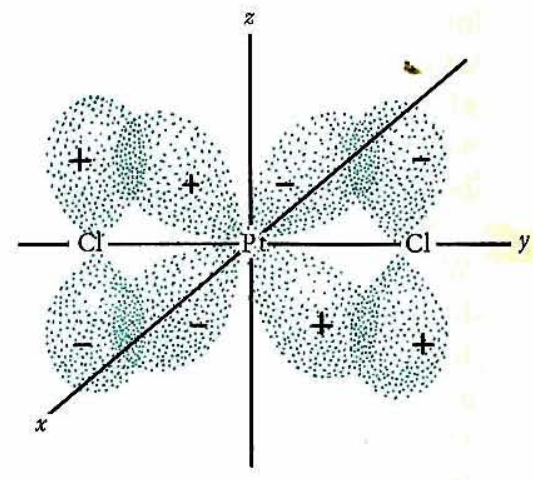

$d_{y z}=d_{x z} \quad(2$ ligand $\pi$ orbitals $)$

Figure 9-14 Overlap of the metal $d$ valence orbitals with the ligand valence orbitals in a square-planar complex. 


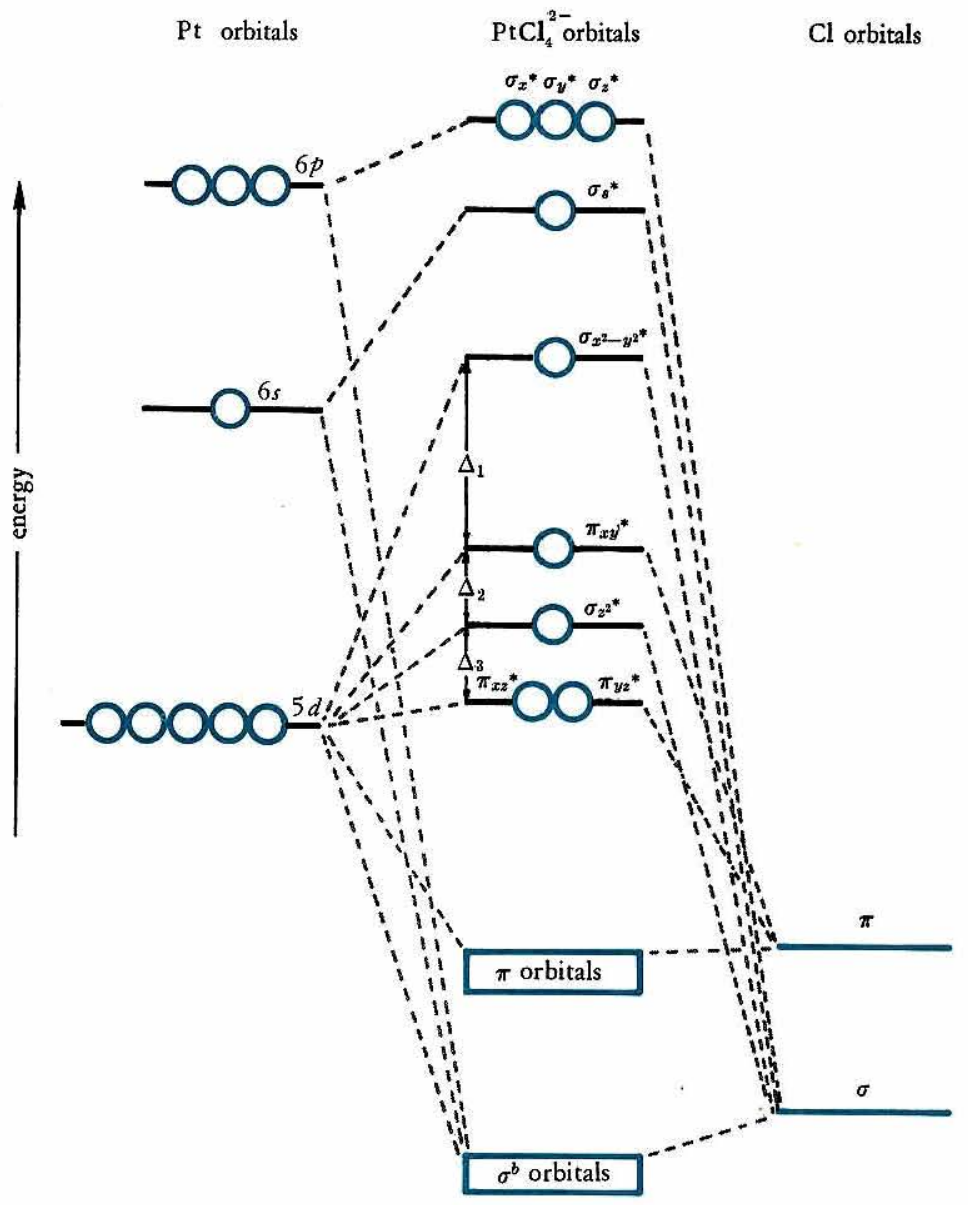

Figure 9-15 Relative orbital energies in $\mathrm{PtCl}_{4}{ }^{2}$.

are paired in the more stable $d$ levels. It is easy to see from the energy-level diagram that the best electronic situation for a squareplanar complex is $d^{8}$. This observation is consistent with the fact that the $d^{8}$ metal ions, among them $\mathrm{Ni}^{2+}, \mathrm{Pd}^{2+}, \mathrm{Pt}^{2+}$, and $\mathrm{Au}^{3+}$, form a great number of square-planar complexes. 


\section{9-11 TETRAHEDRAL COMPLEXES}

A good example of a tetrahedral metal complex is $\mathrm{VCl}_{4}$, the coordinate system for which is shown in Fig. 9-16. We have already discussed the role of $s$ and $p$ valence orbitals in a tetrahedral molecule (Chapter V). The $4 s$ and $4 p$ orbitals of vanadium can be used to form $\sigma$ molecular orbitals. The $3 d_{x z}, 3 d_{y z}$, and $3 d_{x y}$ orbitals are also situated properly for such use. In valence-bond language, $s d^{3}$ and $s p^{3}$ hybrid orbitals are both tetrahedrally directed. The $3 d_{x^{2}-y^{2}}$ and $3 d_{z}{ }^{2}$ orbitals interact very weakly with the ligands to form $\pi$ molecular orbitals.

The simplified molecular-orbital energy-level diagram for $\mathrm{VCl}_{4}$ is shown in Fig. 9-17. Again we place the stable $\sigma$ bonding levels lowest, with the $\pi$ levels, localized on the chlorides, next. The antibonding molecular orbitals derived from the $3 d$ valence orbitals are split into two sets, those based on $3 d_{x z}, 3 d_{y z}$, and $3 d_{x y}$ being less stable than those based on $3 d_{z^{2}}$ and $3 d_{x^{2}-y^{2}}$. We shall designate $\Delta_{t}$ as the difference in energy between $\sigma^{*}(d)$ and $\pi^{*}(d)$ in a tetrahedral complex.

With eight $\sigma$ and sixteen $\pi$ valence electrons from the four chlorides

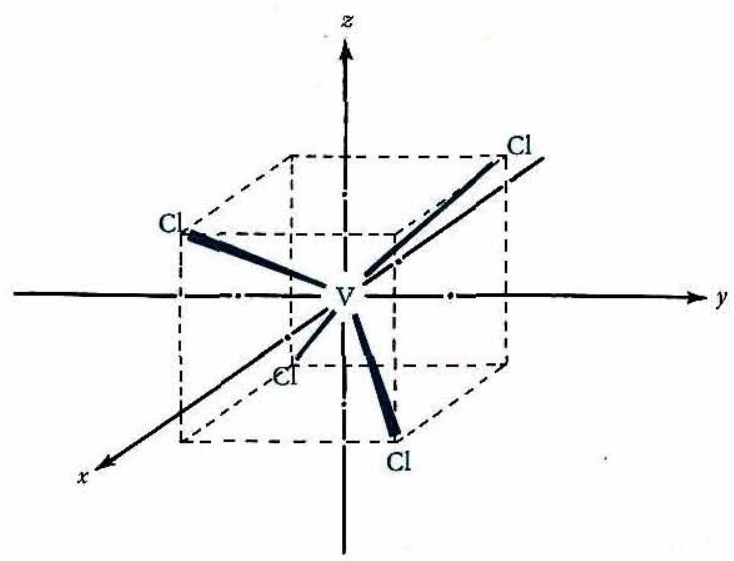

Figure 9-16 Coordinate system for $\mathrm{VCl}_{4}$. 


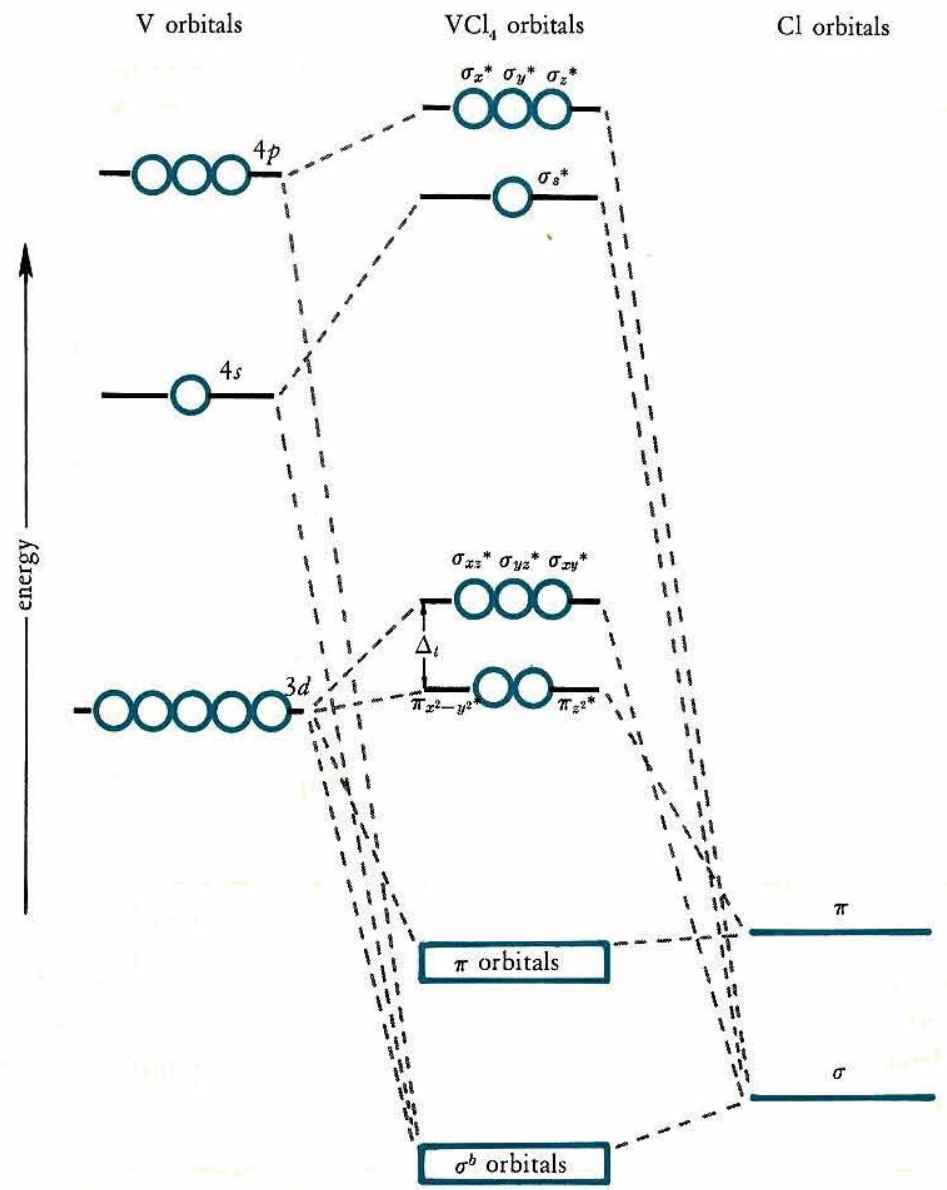

Figure 9-17 Relative orbital energies in $\mathbf{V C I}_{4}$

and with one valence electron from $\mathrm{V}^{4+}\left(3 d^{1}\right)$, the ground state of $\mathrm{VCl}_{4}$ is

$$
\left[\sigma^{b}\right]^{8}[\pi]^{16}\left[\pi^{*}(d)\right]^{1} \quad S=\frac{1}{2}
$$

The paramagnetism of $\mathrm{VCl}_{4}$ is consistent with the ground state, there being one unpaired electron. 
Table $9-1$

Values of $\Delta$ for Representative Metal Complexes

\begin{tabular}{|c|c|c|c|}
\hline $\begin{array}{l}\text { Octahedral } \\
\text { complexes }\end{array}$ & $\Delta, \mathrm{cm}^{-1}$ & $\begin{array}{l}\text { Octahedral } \\
\text { complexes }\end{array}$ & $\Delta, c m^{-1}$ \\
\hline $\mathrm{Ti}\left(\mathrm{H}_{2} \mathrm{O}\right)_{6}{ }^{3+}$ & $20,300^{\mathrm{a}}$ & $\mathrm{Co}\left(\mathrm{H}_{2} \mathrm{O}\right)_{6}{ }^{3+}$ & $18,200^{\mathrm{c}}$ \\
\hline $\mathrm{TiF}_{6}{ }^{3-}$ & $17,000^{b}$ & $\mathrm{Co}\left(\mathrm{NH}_{3}\right)_{6}^{3+}$ & $22,900^{\mathrm{c}}$ \\
\hline $\mathrm{V}\left(\mathrm{H}_{2} \mathrm{O}\right)_{6}{ }^{3+}$ & $17,850^{\mathrm{a}}$ & $\mathrm{Co}(\mathrm{CN})_{6}{ }^{3-}$ & $34,800^{\mathrm{d}}$ \\
\hline $\mathrm{V}\left(\mathrm{H}_{2} \mathrm{O}\right)_{6}{ }^{2+}$ & $12,400^{c}$ & $\mathrm{Co}\left(\mathrm{H}_{2} \mathrm{O}\right)_{6}{ }^{2+}$ & $9,300^{\mathrm{c}}$ \\
\hline $\mathrm{Cr}\left(\mathrm{H}_{2} \mathrm{O}\right)_{6}{ }^{3+}$ & $17,400^{c}$ & $\mathrm{Ni}\left(\mathrm{H}_{2} \mathrm{O}\right)_{6}{ }^{2+}$ & $8,500^{c}$ \\
\hline $\mathrm{Cr}\left(\mathrm{NH}_{3}\right)_{6}{ }^{3+}$ & $21,600^{\mathrm{c}}$ & $\mathrm{Ni}\left(\mathrm{NH}_{3}\right)_{6}{ }^{2+}$ & $10,800^{c}$ \\
\hline $\mathrm{Cr}(\mathrm{CN})_{6}{ }^{3-}$ & $26,600^{d}$ & $\mathrm{RhCl}_{6}{ }^{3-}$ & $20,300^{c}$ \\
\hline $\mathrm{Cr}(\mathrm{CO})_{6}$ & $34,150^{\mathrm{d}}$ & $\mathrm{Rh}\left(\mathrm{NH}_{3}\right)_{6}{ }^{3+}$ & $34 ; 100^{\mathrm{c}}$ \\
\hline $\mathrm{Fe}(\mathrm{CN})_{6}^{3-}$ & $35,000^{d}$ & $\mathrm{RhBr}_{6}{ }^{3-}$ & $19,000^{c}$ \\
\hline \multirow[t]{2}{*}{$\mathrm{Fe}(\mathrm{CN})_{6}^{4-}$} & $33,800^{d}$ & $\operatorname{IrCl}{ }_{6}^{3-}$ & $25,000^{\mathrm{c}}$ \\
\hline & & $\operatorname{Ir}\left(\mathrm{NH}_{3}\right)_{6}{ }^{3+}$ & $40,000^{e}$ \\
\hline \multicolumn{2}{|c|}{ Tetrahedral complexes } & \multicolumn{2}{|c|}{$\Delta, \mathrm{cm}^{-1}$} \\
\hline \multicolumn{2}{|c|}{$\mathrm{VCl}_{4}$} & \multicolumn{2}{|c|}{$9000^{a}$} \\
\hline \multicolumn{2}{|c|}{$\mathrm{CoCl}_{4}{ }^{2-}$} & \multicolumn{2}{|c|}{$3300^{f}$} \\
\hline \multicolumn{2}{|c|}{$\mathrm{CoBr}_{4}^{2-}$} & \multicolumn{2}{|c|}{$2900^{f}$} \\
\hline \multicolumn{2}{|c|}{$\mathrm{CoI}_{4}^{2-}$} & \multicolumn{2}{|c|}{$2700^{f}$} \\
\hline \multicolumn{2}{|c|}{$\mathrm{Co}(\mathrm{NCS})_{4}^{2-}$} & \multicolumn{2}{|c|}{$4700^{f}$} \\
\hline
\end{tabular}

\begin{tabular}{ccccc}
$\begin{array}{c}\text { Square-planar } \\
\text { complexes }\end{array}$ & $\Delta_{1}, \mathrm{~cm}^{-1}$ & $\Delta_{2}, \mathrm{~cm}^{-1}$ & $\Delta_{3}, \mathrm{~cm}^{-1}$ & $\begin{array}{c}\text { Total } \\
\Delta, \mathrm{cm}^{-1}\end{array}$ \\
\hline $\mathrm{PdCl}_{4}{ }^{2-}$ & 19,150 & 6200 & 1450 & 26,800 \\
$\mathrm{PdBr}_{4}{ }^{2-}$ & 18,450 & 5400 & 1350 & 25,200 \\
$\mathrm{PtCl}_{4}{ }^{2-}$ & 23,450 & 5900 & 4350 & 33,700 \\
$\mathrm{PtBr}_{4}{ }^{2-}$ & 22,150 & 6000 & 3550 & 31,700 \\
$\mathrm{Ni}(\mathrm{CN})_{4}{ }^{2-}$ & 24,950 & 9900 & 650 & 35,500 \\
\hline
\end{tabular}


Excitation of the electron in $\pi^{*}(d)$ to $\sigma^{*}(d)$ is accompanied by light absorption, with a maximum at $9000 \mathrm{~cm}^{-1}$. Thus $\Delta_{t}$ for $\mathrm{VCl}_{4}$ is $9000 \mathrm{~cm}^{-1}$.

\section{9-12 THE VALUE OF $\Delta$}

The splitting of the molecular orbitals derived from metal $d$ valence orbitals involves a quantity that is of considerable interest when discussing the electronic structures of metal complexes. The $\Delta$ values for a representative selection of octahedral, square-planar, and tetrahedral complexes are given in Table 9-1. The value of $\Delta$ depends on a number of variables, the most important being the geometry of the complex, the nature of the ligand, the charge on the central metal ion, and the principal quantum number $n$ of the $d$ valence orbitals. We shall discuss these variables individually.

\section{Geometry of the Complex}

By extrapolating the data in Table 9-1, we may estimate that, other things being equal, the total $d$-orbital splitting decreases as follows:

$$
\begin{array}{ccc}
\text { square planar } & >\text { octahedral } & >\text { tetrahedral } \\
1.3 \Delta_{0} & \Delta_{0} & 0.45 \Delta_{0}
\end{array}
$$

In the molecular-orbital theory, the $d$-orbital splitting is interpreted as the difference between the strengths of $\sigma$ and $\pi$ bonding as measured by the difference in energy between the $\sigma^{*}$ and $\pi$ (or $\pi^{*}$ ) molecular orbitals. The tetrahedral splitting is smallest because the $d$ orbitals are not involved in strong $\sigma$ bonding. In both octahedral and square-planar complexes, $d$ orbitals are involved in strong $\sigma$ bond-

${ }^{a}$ C. J. Ballhausen, Introduction to Ligand Field Theory, McGraw-Hill, New York, 1962, Chap. 10.

${ }^{b}$ H. Bedon, S. M. Horner, and S. Y. Tyree, Inorg. Chem., 3, 647 (1964).

${ }^{\mathrm{c}} \mathrm{C} . \mathrm{K}$. Jørgensen, Absorption Spectra and Chemical Bonding, Pergamon, London, 1962, Table 11.

dH. B. Gray and N. A. Beach, J. Am. Chem. Soc., 85, 2922 (1963).

${ }^{\mathrm{e}}$ H. B. Gray, unpublished results.

f Averaged from values in Ref. c and in F. A. Cotton, D. M. L. Goodgame, and M. Goodgame, J.Am.Chem. Soc., 83, 4690 (1961).

${ }^{8}$ H. B. Gray and C. J. Ballhausen, J. Am. Chem. Soc.s 85, 260 (1963). 
ing, but the total squate-planar splitting $\left(\Delta_{1}+\Delta_{2}+\Delta_{3}\right)$ will always be larger than the octahedral splitting since the $d_{x z}$ and $d_{y z}$ orbitals interact with only two ligands in a square-planar complex (as opposed to four in an octahedral complex; see Fig. 9-11).

\section{Nature of the Ligand: the Spectrochemical Series}

The spectrochemical series represents the ordering of ligands in terms of their ability to split the $\sigma^{*}(d)$ and $\pi(d)$ molecular orbitals. Complexes containing ligands such as $\mathrm{CN}^{-}$and $\mathrm{CO}$, which are high in the spectrochemical series, have $\Delta$ values in the range of 30,000 $\mathrm{cm}^{-1}$. At the other end of the series, $\mathrm{Br}^{-}$and $\mathrm{I}^{-}$cause very small splittings-in many cases less than $10,000 \mathrm{~cm}^{-1}$. We have already discussed the important types of metal-ligand bonding in transitionmetal complexes. The manner in which each type affects the value of $\Delta$ is illustrated in Fig. 9-18. We see that a strong ligand-to-metal $\sigma$ interaction destabilizes $\sigma^{*}(d)$, increasing the value of $\Delta$. A strong $L \rightarrow M \pi$ interaction destabilizes $\pi(d)$, decreasing the value of $\Delta$. A strong $M \rightarrow L \pi$ interaction stabilizes $\pi(d)$, increasing the value of $\Delta$. It is striking that the spectrochemical series correlates reasonably

metal orbitals

molecular orbitals

ligand orbitals

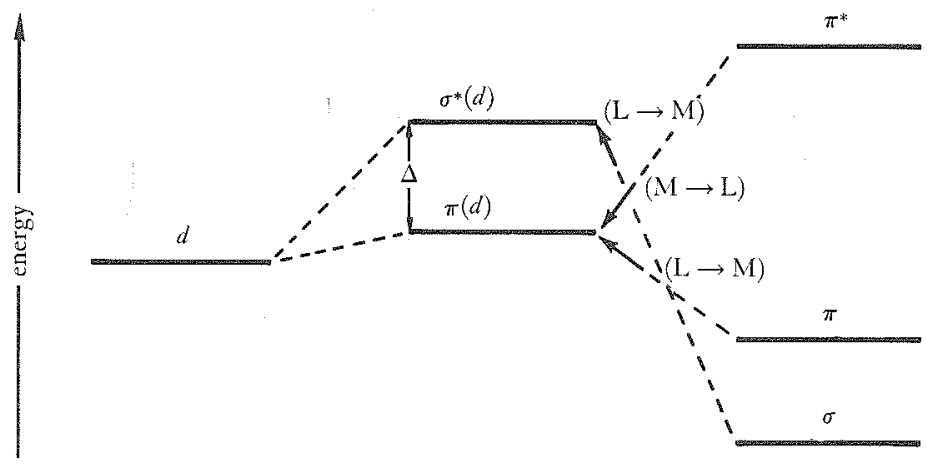

Figure 9-18 The effect of interaction of the ligand $\sigma$, $\pi$, and $\pi^{*}$ orbitals on the value of $\Delta$. 
well with the $\pi$-bonding abilities of the ligands. The good $\pi$ acceptor ligands (those capable of strong $M \rightarrow L \pi$ bonding) cause large splittings, whereas the good $\pi$-donor ligands (those capable of strong $L \rightarrow M \pi$ bonding) cause small splittings. The ligands with intermediate $\Delta$ values have little or no $\pi$-bonding capabilities.

The spectrochemical-series order of some important ligands is indicated below:

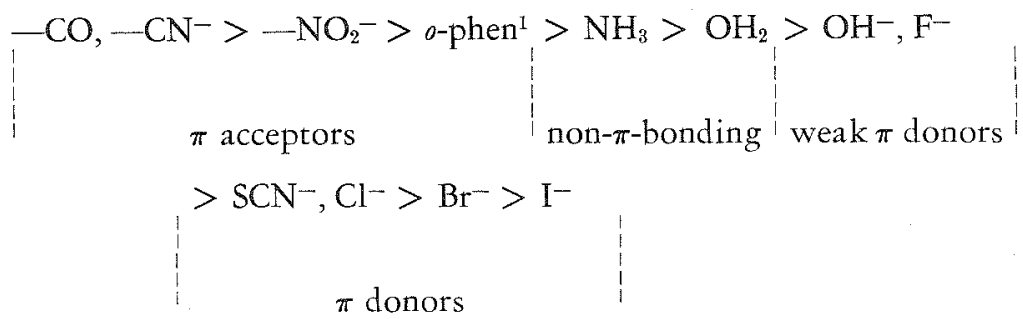

\section{Charge on the Central Metal Ion}

In complexes containing ligands that are not good $\pi$ acceptors, $\Delta$ increases with increasing positive charge on the central metal ion. A good example is the comparison between $\mathrm{V}\left(\mathrm{H}_{2} \mathrm{O}\right)_{6}{ }^{2+}$, with $\Delta=$ $11,800 \mathrm{~cm}^{-1}$, and $\mathrm{V}\left(\mathrm{H}_{2} \mathrm{O}\right)_{6}{ }^{8+}$, with $\Delta=17,850 \mathrm{~cm}^{-1}$. The increase in $\Delta$ in these cases is interpreted as a substantial increase in $\sigma$ bonding on increasing the positive charge of the central metal jon. This would result in an increase in the difference in energy between $\sigma^{*}(d)$ and $\pi(d)$.

In complexes containing good $\pi$-acceptor ligands, an increase in positive charge on the metal does not seem to be accompanied by a substantial increase in $\Delta$. For example, both $\mathrm{Fe}(\mathrm{CN})_{6}{ }^{4-}$ and $\mathrm{Fe}(\mathrm{CN})_{6}{ }^{3-}$ have $\Delta$ values of approximately $34,000 \mathrm{~cm}^{-1}$. In the transition from $\mathrm{Fe}(\mathrm{CN})_{6}{ }^{4-}$ to $\mathrm{Fe}(\mathrm{CN})_{6}{ }^{3-}$, the $\pi(d)$ level is destabilized just as much as the $\sigma^{*}(d)$ level, probably the result of a decrease in $M \rightarrow L \pi$ bonding when the positive charge on the metal ion is increased.

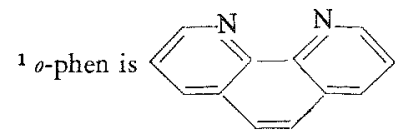




\section{Principal Quantum Number of the d Valence Orbitals}

In an analogous series of complexes, the value of $\Delta$ varies with $n$ in the $d$ valence orbitals as follows: $3 d<4 d<5 d$. For example, the $\Delta$ values for $\mathrm{Co}\left(\mathrm{NH}_{3}\right)_{6}{ }^{3+}, \mathrm{Rh}\left(\mathrm{NH}_{3}\right)_{6}{ }^{3+}$, and $\operatorname{Ir}\left(\mathrm{NH}_{3}\right)_{6}{ }^{3+}$ are 22,900, 34,100 , and $40,000 \mathrm{~cm}^{-1}$, respectively. Presumably the $5 d$ and $4 d$ valence orbitals are better than the $3 d$ in $\sigma$ bonding with the ligands.

\section{9-13 THE MAGNETIC PROPERTIES OF COMPLEXES: WEAK- AND STRONG-FIELD LIGANDS}

We shall now consider in some detail the ground-state electronic configurations of octahedral complexes containing metal ions with more than one valence electron. Referring back to Fig. 9-5, we see that metal ions with one, two, and three valence electrons will have the respective ground-state configurations $\pi(d), S=\frac{1}{2} ;[\pi(d)]^{2}, S=$ 1 ; and $[\pi(d)]^{3}, S=\frac{3}{2}$. There are two possibilities for the metal $d^{4}$ configuration, depending on the value of $\Delta$ in the complex. If $\Delta$ is less than the energy required to pair two $d$ electrons in the $\pi(d)$ level, the fourth electron will go into the $\sigma^{*}(d)$ level, giving the configuration $[\pi(d)]^{3}\left[\sigma^{*}(d)\right]^{1}$ and four unpaired electrons $(S=2)$. Ligands that cause such small splittings are called weak-field ligands.

On the other hand, if $\Delta$ is larger than the required pairing energy, the fourth electron will prefer to go into the more stable $\pi(d)$ level and pair with one of the three electrons already present in this level. The ground-state configuration of the complex in this situation is $[\pi(d)]^{4}$, with only two unpaired electrons $(S=1)$. Ligands that cause splittings large enough to allow electrons to preferentially occupy the more stable $\pi(d)$ level are called strong-field ligands.

It is clear that, in filling the $\pi(d)$ and $\sigma^{*}(d)$ levels, the configurations $d^{4}, d^{5}, d^{6}$, and $d^{7}$ can have either of two possible values of $S$, depending on the value of $\Delta$ in the complex. When there is such a choice, the complexes with the larger $S$ values are called bigh-spin complexes, and those with smaller $S$ values are called low-spin complexes. The paramagnetism of the high-spin complexes is larger than that of the low-spin complexes. Examples of octahedral complexes with the possible $[\pi(d)]^{x}\left[\sigma^{*}(d)\right]^{y}$ configurations are given in Table 9-2. 


$$
\text { Table } 9-2
$$

Electronic Configurations of Octahedral Complexes

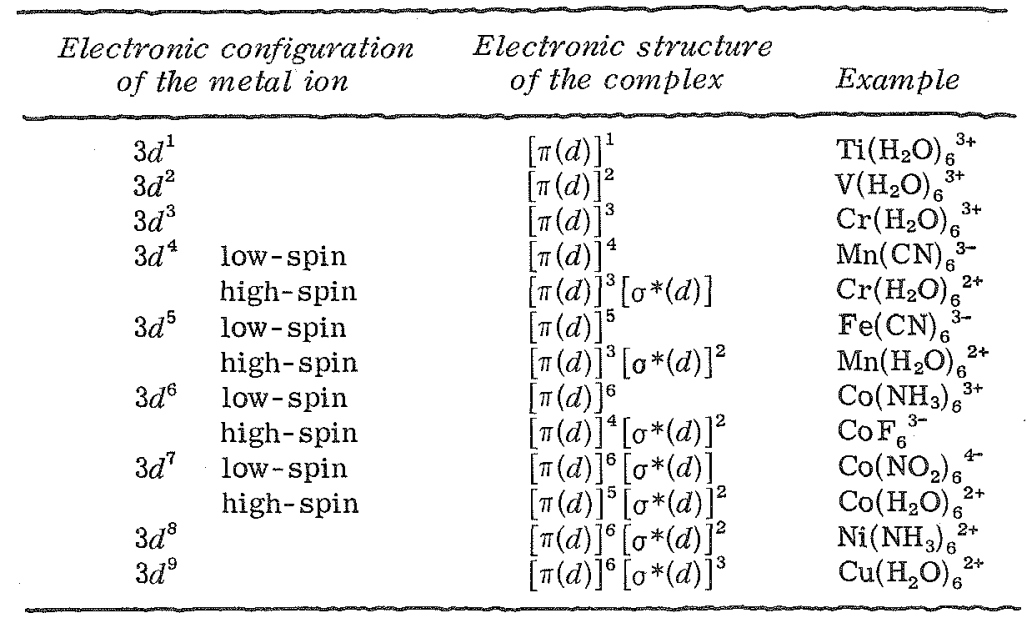

The first-row transition-metal ions that form the largest number of stable octahedral complexes are $\mathrm{Cr}^{3+}\left(d^{3}\right), \mathrm{Ni}^{2+}\left(d^{8}\right)$, and $\mathrm{Co}^{3+}\left(d^{6}\right.$; lowspin). This observation is consistent with the fact that the MO configurations $[\pi(d)]^{3}$ and $[\pi(d)]^{6}$ take maximum advantage of the more stable $\pi(d)$ level. The $[\pi(d)]^{6}\left[\sigma^{*}(d)\right]^{2}$ configuration is stable for relatively small $\Delta$ values.

The splitting for the tetrahedral geometry is always small, and no low-spin complexes are known for first-row transition-metal ions. There are many stable tetrahedral complexes of $\mathrm{Co}^{2+}\left(3 d^{7}\right)$, among them $\mathrm{CoCl}_{4}{ }^{2-}, \mathrm{Co}(\mathrm{NCS})_{4}{ }^{2-}$, and $\mathrm{Co}(\mathrm{OH})_{4}{ }^{2-}$. This is consistent with the fact that the $\left[\pi^{*}(d)\right]^{4}\left[\sigma^{*}(d)\right]^{3}$ configuration makes maximum use of the more stable $\pi^{*}(d)$ level.

\section{9-14 THE ELECTRONIC SPECTRA OF OCTAFEDRAL COMPLEXES}

The $\mathrm{Ti}\left(\mathrm{H}_{2} \mathrm{O}\right)_{6}{ }^{3+}$ spectrum is simple, since the only $d-d$ transition possible is $\pi(d) \rightarrow \sigma^{*}(d)$. We must now consider how many absorp- 
tion bands can be expected in complexes containing metal ions with more than one $d$ electron. One simple and useful method is to calculate the splitting of the free-ion terms in an octahedral crystal field. As an example, consider the spectrum of $\mathrm{V}\left(\mathrm{H}_{2} \mathrm{O}\right)_{6}{ }^{2+}$.

The valence electronic configuration of $\mathrm{V}^{2+}$ is $3 d^{3}$. The free-ion terms for $d^{3}$ are obtained as outlined in Chapter I; they are ${ }^{4} F,{ }^{4} P$, ${ }^{2} G,{ }^{2} D$, and ${ }^{2} S$, the ground state being ${ }^{4} F$ according to Hund's rules.
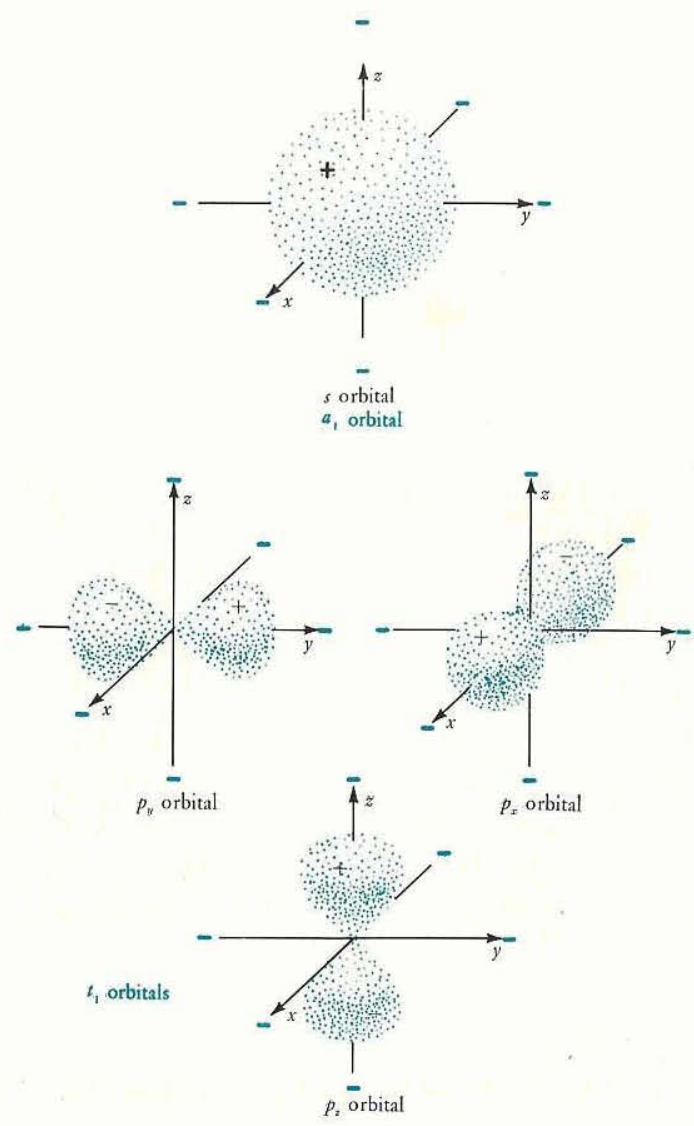

Figure 9-19 Splittings of the $s, p, d$, and $f$ orbitals in an octahedral crystal field. 

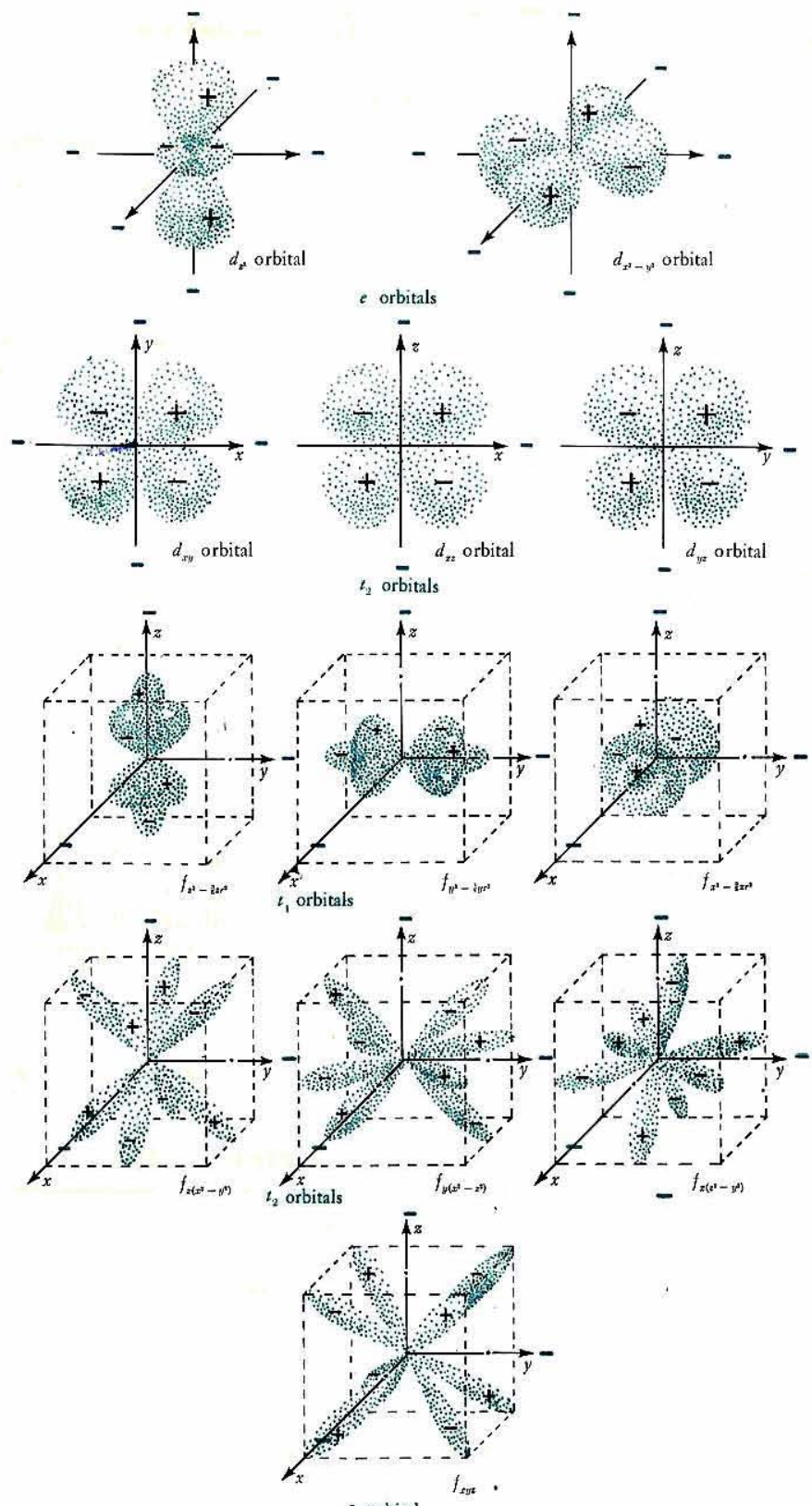

$a_{2}$ orbital

Figure 9-19 (rontinued) 
Since transitions between states that have different $S$ values are forbidden (referred to as spin-forbidden), we shall consider the splitting of only the ${ }^{4} F$ and ${ }^{4} P$ terms in the octahedral field. In order to determine this splitting, we make use of the fact that the free-ion terms and the single-electron orbitals with the same angular momentum split up into the same number of levels in a crystal field. That is, a $D$ term splits into two levels, which we call $T_{2}$ and $E$, just as the $d$ orbitals split into $t_{2}$ and $e$ levels.

The $s, p, d$, and $f$ orbitals are shown in an octahedral field in Fig. 9-19. The splittings we deduce from Fig. 9-19 are summarized in Table 9-3. We see that the ${ }^{4} F$ term splits into three levels, ${ }^{4} A_{2},{ }^{4} T_{2}$, and ${ }^{4} T_{1}$; the ${ }^{4} P$ term does not split, but simply gives a ${ }^{4} T_{1}$ level.

The energy-level diagram appropriate for a discussion of the spectrum of $\mathrm{V}\left(\mathrm{H}_{2} \mathrm{O}\right)_{6}{ }^{2+}$ is shown in Fig. 9-20. The ${ }^{4} P$ term is placed higher than ${ }^{4} F$, following Hund's second rule. The ${ }^{4} P$ term is known to be $11,500 \mathrm{~cm}^{-1}$ above the ${ }^{4} F$ term in the $\mathrm{V}^{2+}$ ion. A calculation is required in order to obtain the relative energies of the three levels produced from the ${ }^{4} F$ term. The results are given in Fig. 9-20 in terms of the octahedral splitting parameter $\Delta$.

The ground state of $\mathrm{V}\left(\mathrm{H}_{2} \mathrm{O}\right)_{6}{ }^{2+}$ is ${ }^{4} A_{2}$. From the diagram, we see that there are three transitions possible: ${ }^{4} A_{2} \rightarrow{ }^{4} T_{2} ;{ }^{4} A_{2} \rightarrow{ }^{4} T_{1}(F)$; and ${ }^{4} A_{2} \rightarrow{ }^{4} T_{1}(P)$. The spectrum of $\mathrm{V}\left(\mathrm{H}_{2} \mathrm{O}\right)_{6}{ }^{2+}$ is shown in Fig. 9-21. There are three bands, in agreement with the theoretical prediction.

Table $9-3$

Splittings Deduced from Figure $9-19$

\begin{tabular}{cccc}
\hline $\begin{array}{c}\text { Orbital } \\
\text { Set }\end{array}$ & $\begin{array}{c}\text { Number of } \\
\text { levels }\end{array}$ & $\begin{array}{c}\text { Level } \\
\text { notation }\end{array}$ & $\begin{array}{c}\text { Level } \\
\text { degeneracy }\end{array}$ \\
\hline \multirow{2}{*}{$s$} & 1 & $a_{1}$ & 1 \\
$p$ & 1 & $t_{1}$ & 3 \\
$d$ & 2 & $t_{2}$ & 3 \\
& & $e$ & 2 \\
$f$ & 3 & $a_{2}$ & 1 \\
& & $t_{2}$ & 3 \\
& & $t_{1}$ & 3 \\
\hline
\end{tabular}




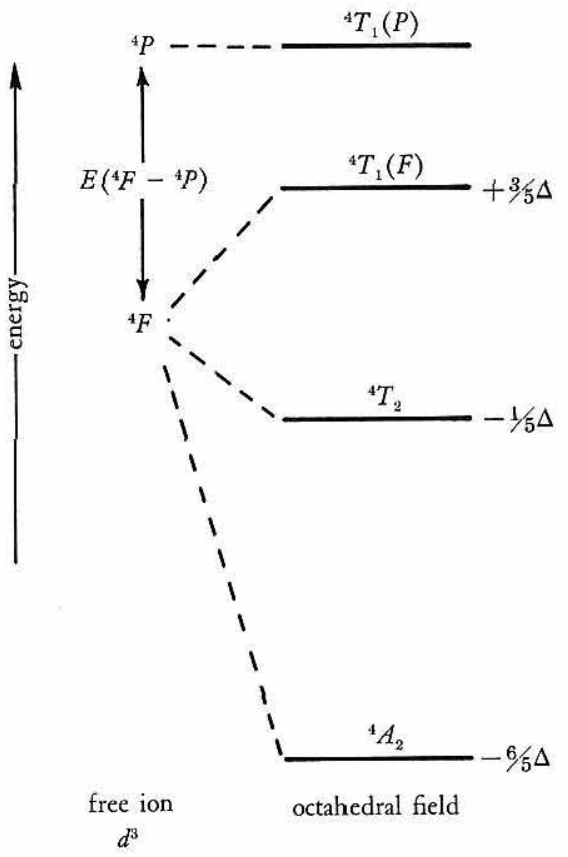

Figure 9-20 Energy-level diagram for a $d^{3}$ metal ion in an octahedral field.

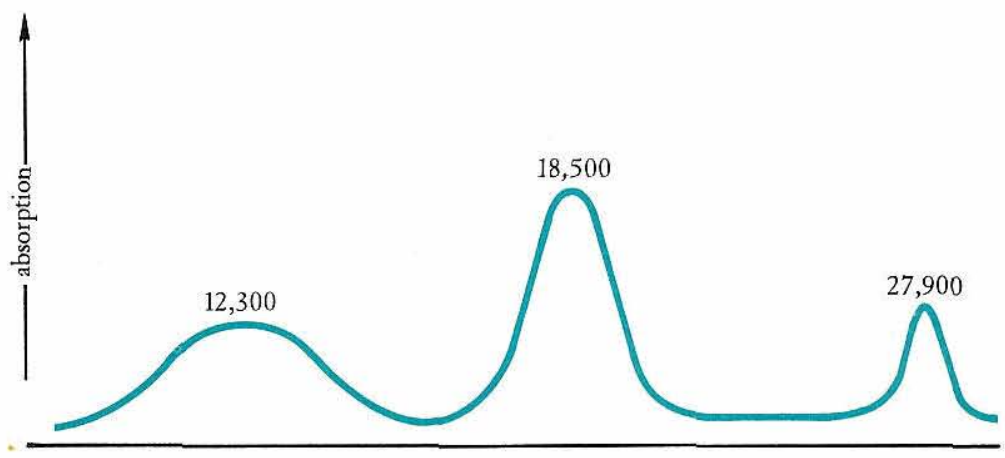

$\bar{\nu}, \mathrm{cm}^{-1}$

Figure 9-21 Electronic absorption spectrum of $\mathrm{V}\left(\mathrm{H}_{2} \mathrm{O}\right)_{6}{ }^{2+}$. 
Table $9-\frac{4}{2}$

Energy Expressions for the Three Posslble Transtlions of $\mathrm{V}\left(\mathrm{H}_{2} \mathrm{O}\right){ }^{2+}$

\begin{tabular}{cc}
\hline Transition & \multicolumn{1}{c}{ Energy } \\
\hline${ }^{4} A_{2} \rightarrow{ }^{4} T_{2}$ & $\Delta$ \\
${ }^{4} A_{2} \rightarrow{ }^{4} T_{1}(F)$ & $\frac{9}{5} \Delta$ \\
${ }^{4} A_{2} \rightarrow{ }^{4} T_{1}(P)$ & $\frac{6}{5} \Delta+E\left({ }^{4} F-{ }^{4} P\right)$ \\
\hline
\end{tabular}

According to the energy-level diagram, the energies of the transitions are those listed in Table 9-4.

Assigning the first band at $12,300 \mathrm{~cm}^{-1}$ to the ${ }^{4} A_{2} \rightarrow{ }^{4} T_{2}$ transition, we obtain $\Delta=12,300 \mathrm{~cm}^{-1}$. Using $\Delta=12,300 \mathrm{~cm}^{-1}$ and $E\left({ }^{4} F-{ }^{4} \mathrm{P}\right)=11,500 \mathrm{~cm}^{-1}$ for $\mathrm{V}\left(\mathrm{H}_{2} \mathrm{O}\right)_{6}{ }^{2+}$, the other two transition energies can be calculated and compared with experiment as shown in Table 9-5.

The appropriate energy-level diagrams for several important $d$ electron configurations are given in Fig. 9-22.

Table $8-5$

Comparison between Calculated and Observed Transition Energles for $\mathrm{V}\left(\mathrm{H}_{2} \mathrm{O}\right)_{6}{ }^{2+}$

\begin{tabular}{ccc}
\hline & \multicolumn{2}{c}{ Energy values, $\mathrm{cm}^{-1}$} \\
\cline { 2 - 3 } Transition & Calculated & observed $^{\mathrm{a}}$ \\
\hline${ }^{4} A_{2} \rightarrow{ }^{4} T_{2}$ & $(12,300)$ & 12,300 \\
${ }^{4} A_{2} \rightarrow{ }^{4} T_{1}(F)$ & 22,140 & 18,500 \\
${ }^{4} A_{2} \rightarrow{ }^{4} T_{1}(P)$ & 26,260 & 27,900 \\
\hline
\end{tabular}

${ }^{\mathrm{a}} \mathrm{C}$. K. Jørgensen, Absorption Spectra and Chemical Bonding, Pergamon, London, 1962, p. 290. 


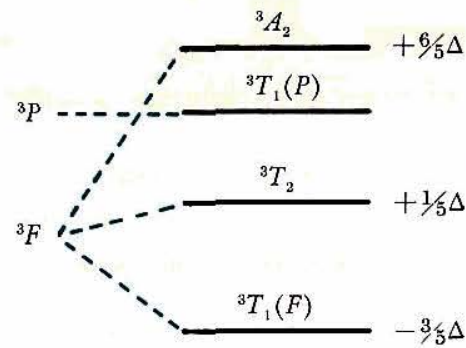

free ion $d^{2}$

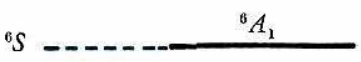

free ion

$d^{5}$
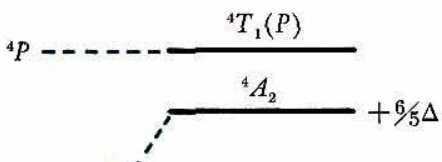

${ }^{4} F$

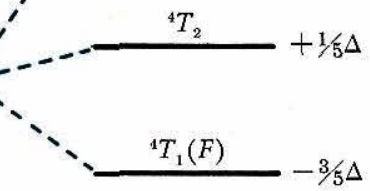

free ion $d^{7}$

octahedral field

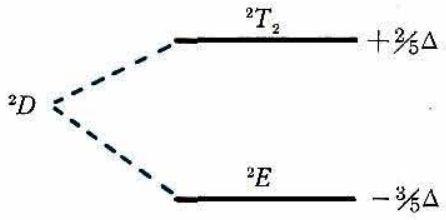

free ion

octahedral field

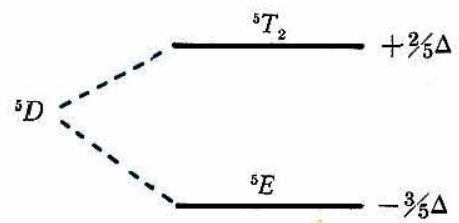

free ion octahedral field $d^{4}$

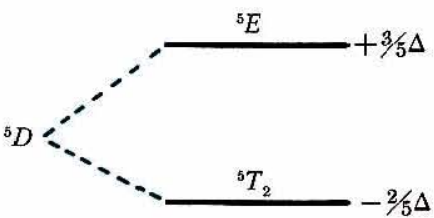

free ion octahedral field $d^{6}$

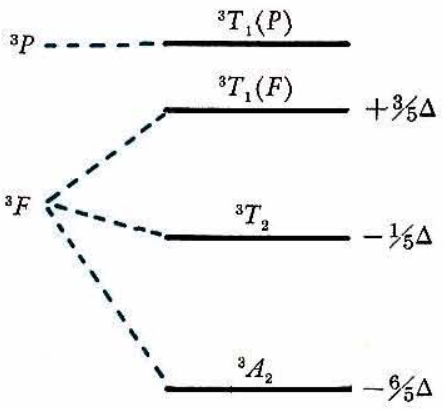

free ion

octahedral field $d^{8}$

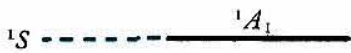

free ion

octahedral field $d^{9}$ $d^{10}$

Figure 9-22 Energy-level diagrams for the $d^{n}$ metal ions in an octahedral field. 


\section{PROBLEM}

9-1. Show that the $d_{z^{2}}$ and $d_{x^{2}-y^{2}}$ orbitals are equivalent in an octahedral complex.

Solution. We shall solve this problem by calculating the total overlap of the $d_{x^{2}}-y^{2}$ and $d_{z}{ }^{2}$ orbitals with their respective normalized ligand-orbital combinations. The total overlap in each case, $S\left(d_{x^{2}-y^{2}}\right)$ and $S\left(d_{z^{2}}\right)$, will be expressed in terms of the standard twoatom overlap between $d_{z}{ }^{2}$ and a ligand $\sigma$ orbital, as shown in Fig. 9-23. This overlap is called $S\left(\sigma, d_{\sigma}\right)$. From Table 1-1, we see that the angular functions for $d_{x^{2}-y^{2}}$ and $d_{x^{2}}$ are

and

$$
d z^{2}=c\left(3 z^{2}-r^{2}\right)
$$

with $c=\sqrt{5} /\left(4 \sqrt{\pi}-d_{x^{2}-y^{2}}=\sqrt{3 c}\left(x^{2}-y^{2}\right)\right.$

with $c=\sqrt{5} /\left(4 \sqrt{\pi} r^{2}\right)$. The normalized combinations of ligand orbitals are

and

$$
d_{z^{2}}: \quad \frac{1}{2 \sqrt{3}}\left(2 z_{5}+2 z_{6}-z_{1}-z_{2}-z_{3}-z_{4}\right)
$$

$$
d_{x^{2}-y^{2}}: \quad \frac{1}{2}\left(z_{1}-z_{2}+z_{3}-z_{4}\right)
$$

We first evaluate $S\left(d_{x^{2}-y^{2}}\right)$ :

$$
S\left(d_{x^{2}-y^{2}}\right)=\int \sqrt{3} c\left(x^{2}-y^{2}\right) \frac{1}{2}\left(z_{1}-z_{2}+z_{3}-z_{4}\right) d \tau
$$

This integral is transformed into the standard two-atom overlap integral $S\left(\sigma, d_{\sigma}\right)$ by rotating the metal coordinate system to coincide in turn with the coordinate systems of ligands (1), (2), (3), and (4).

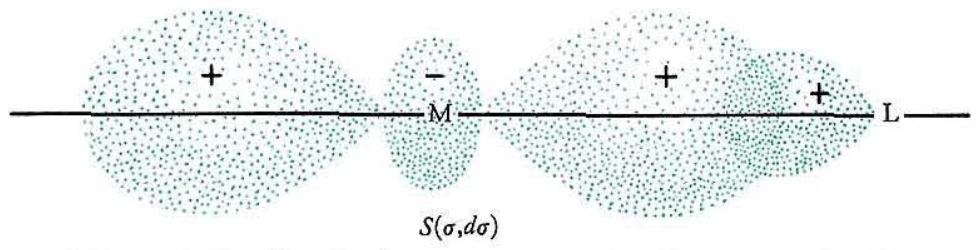

Figure 9-23 Standard two-atom $\sigma$ overlap between a $d$ and a ligand $\sigma$ valence orbital. 
Using the coordinates shown in Fig. 9-24, we obtain the following transformations:

\begin{tabular}{|c|c|c|c|}
\hline$M$ to (1) & $M$ to (2) & $M$ to $(3)$ & $M$ to (4) \\
\hline$z \rightarrow \quad y$ & $z \rightarrow \quad x$ & $z \rightarrow-x$ & $z \rightarrow-y$ \\
\hline$x \rightarrow-z$ & $x \rightarrow y$ & $x \rightarrow z$ & $x \rightarrow-x$ \\
\hline$y \rightarrow x$ & $y \rightarrow-z$ & $y \rightarrow-y$ & $y \rightarrow$ \\
\hline
\end{tabular}

Thus we have:

$$
\begin{aligned}
\frac{\sqrt{3}}{2} c\left(x^{2}-y^{2}\right) z_{1} & \rightarrow \frac{\sqrt{3}}{2} c\left(z^{2}-x^{2}\right) \sigma \\
-\frac{\sqrt{3}}{2} c\left(x^{2}-y^{2}\right) z_{2} & \rightarrow-\frac{\sqrt{3}}{2} c\left(y^{2}-z^{2}\right) \sigma \\
\frac{\sqrt{3}}{2} c\left(x^{2}-y^{2}\right) z_{3} & \rightarrow \frac{\sqrt{3}}{2} c\left(z^{2}-y^{2}\right) \sigma \\
-\frac{\sqrt{3}}{2} c\left(x^{2}-y^{2}\right) z_{4} & \rightarrow-\frac{\sqrt{3}}{2} c\left(x^{2}-z^{2}\right) \sigma
\end{aligned}
$$

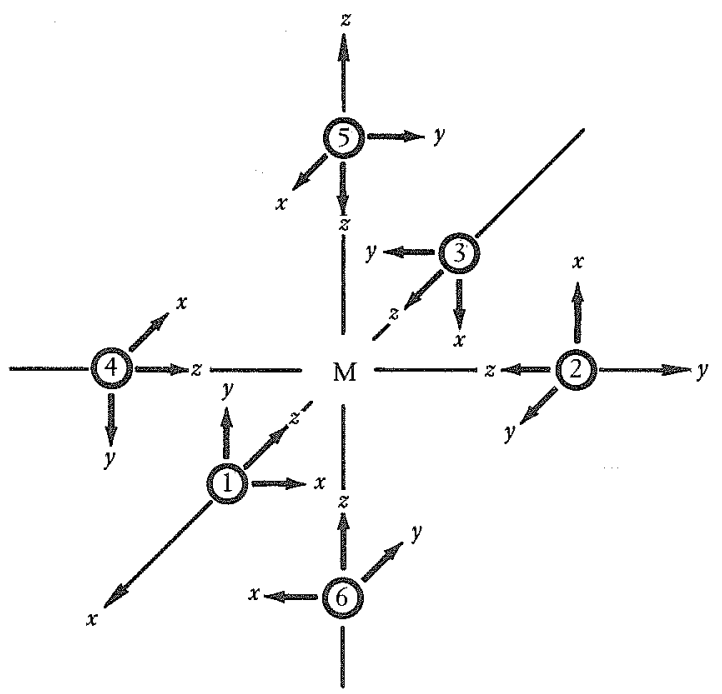

Figure 9-24 Coordinate system for an octahedral complex. 
Adding the four transformed terms, we have

$$
\begin{aligned}
S\left(d_{z^{2}-y^{2}}\right) & =\int \sqrt{3} c\left(2 z^{2}-x^{2}-y^{2}\right) \sigma d \tau=\int \sqrt{3} c\left(3 z^{2}-r^{2}\right) \sigma d r \\
& =\sqrt{3} S\left(\sigma, d_{\sigma}\right)
\end{aligned}
$$

Next we evaluate $S\left(d_{z}^{2}\right)$ :

$$
S\left(d_{z^{2}}\right)=\int c\left(3 z^{2}-r^{2}\right) \frac{1}{2 \sqrt{3}}\left(2 z_{5}+2 z_{6}-z_{1}-z_{2}-z_{3}-z_{4}\right) d \tau
$$

The integrals involving $z_{5}$ and $z_{6}$ are simply two-atom overlaps, as shown in Fig. 9-23. Thus we have

$$
\int c\left(3 z^{2}-r^{2}\right) \frac{1}{2 \sqrt{3}}\left(2 z_{5}+2 z_{6}\right) d \tau=\frac{2}{\sqrt{3}} S\left(\sigma, d_{\sigma}\right)
$$

The integral involving $z_{1}, z_{2}, z_{3}$, and $z_{4}$ is transformed into $S\left(\sigma, d_{\sigma}\right)$, using the transformation table that was used for $S\left(d_{x^{2}}-y^{2}\right)$. Thus

$$
\begin{aligned}
& -r\left(3 z^{2}-r^{2}\right) z_{1} \rightarrow-c\left(3 y^{2}-r^{2}\right) \sigma \\
& -c\left(3 z^{2}-r^{2}\right) z_{2} \rightarrow-c\left(3 x^{2}-r^{2}\right) \sigma \\
& -c\left(3 z^{2}-r^{2}\right) z_{3} \rightarrow-c\left(3 x^{2}-r^{2}\right) \sigma \\
& -c\left(3 z^{2}-r^{2}\right) z_{4} \rightarrow-c\left(3 y^{2}-r^{2}\right) \sigma
\end{aligned}
$$

Totaling the four transformed terms, we find

$$
\begin{aligned}
\int c\left(3 z^{2}-r^{2}\right) \frac{1}{2 \sqrt{3}}\left(-z_{1}-z_{2}-z_{3}-z_{4}\right) d \tau & \\
=\frac{1}{2 \sqrt{3}} \int c\left(6 x^{2}+6 y^{2}-4 r^{2}\right) \sigma d \tau & =\frac{1}{\sqrt{3}} \int c\left(3 z^{2}-r^{2}\right) \sigma d \tau \\
& =\frac{1}{\sqrt{3}} S\left(\sigma, d_{\sigma}\right)
\end{aligned}
$$

Finally, combining the results of Eqs. (9-25) and (9-30), we obtain

$$
S\left(d_{2}^{2}\right)=\frac{2}{\sqrt{3}} S\left(\sigma, d_{\sigma}\right)+\frac{1}{\sqrt{3}} S\left(\sigma, d_{\sigma}\right)=\sqrt{3} S\left(\sigma, d_{\theta}\right)
$$

Then

$$
S\left(d_{z^{2}}\right)=S\left(d_{x^{2}-y^{2}}\right)=\sqrt{3} S\left(\sigma, d_{\sigma}\right)
$$


Thus the total overlap of $d_{x^{2}-y^{2}}$ and $d_{z} z^{2}$ with properly normalized ligand-orbital combinations is the same, and it follows that the two orbitals are equivalent in an octahedral complex.

\section{SUPPLEMENTARY PROBLEMS}

1. Under what conditions are the molecular-orbital and valencebond descriptions of the $\sigma$ bonding in an octahedral complex equivalent? Derive the valence-bond functions shown in Fig. 9-7 from the general molecular-orbital functions.

2. Construct the molecular-orbital and valence-bond wave functions for the $\sigma$ bonding in a square-planar complex. When are the molecular-orbital and valence-bond descriptions the same?

3. Which complex has the larger $\Delta$ value, $\operatorname{Co}(\mathrm{CN})_{6}{ }^{3-}$ or $\mathrm{Co}\left(\mathrm{NH}_{3}\right)_{6}{ }^{3+}$ ? $\mathrm{Co}\left(\mathrm{NH}_{3}\right)_{6}^{3+}$ or $\mathrm{CoF}_{6}^{3-?} \mathrm{Co}\left(\mathrm{H}_{2} \mathrm{O}\right)_{6}{ }^{3+}$ or $\mathrm{Rh}\left(\mathrm{H}_{2} \mathrm{O}\right)_{6}{ }^{3+}$ ? $\mathrm{PdCl}_{4}{ }^{2-}$ or $\mathrm{PrCl}_{4}{ }^{2-}$ ? $\mathrm{PrI}_{4}{ }^{2-}$ or $\mathrm{PrCl}_{4}{ }^{2-}$ ? $\mathrm{VCl}_{4}$ or $\mathrm{CoCl}_{4}{ }^{2-}$ ? $\mathrm{VCl}_{4}$ or $\mathrm{CoF}_{6}{ }^{3-}$ ? $\mathrm{PdCl}_{4}{ }^{2-}$ or $\mathrm{RhCl}_{6}{ }^{3-}$ ? $\mathrm{Co}\left(\mathrm{H}_{2} \mathrm{O}\right)_{6}{ }^{2+}$ or $\mathrm{Co}\left(\mathrm{H}_{2} \mathrm{O}\right)_{6}{ }^{3+}$ ?

4. Give the number of unpaired electrons for each of the following complexes: (a) $\mathrm{VF}_{6}{ }^{3-}$; (b) $\mathrm{FeCl}_{4}^{-}$; (c) $\mathrm{NiCl}_{4}{ }^{2-}$ (tetrahedral); (d) $\mathrm{PdCl}_{4}{ }^{2-}$; (e) $\mathrm{Cu}\left(\mathrm{NH}_{3}\right)_{4}{ }^{2+} ;$ (f) $\mathrm{Fe}(\mathrm{CN})_{6}{ }^{4-} ;$ (g) $\mathrm{Fe}(\mathrm{CN})_{6}{ }^{3-}$; (b) $\mathrm{TiF}_{6}{ }^{3-}$; (i) $\mathrm{Ni}\left(\mathrm{CN}_{4}{ }^{2-}\right.$; (j) $\mathrm{RhCl}_{6}{ }^{3-}$; (k) $\mathrm{IrCl}_{6}{ }^{2-}$.

5. Explain why $\mathrm{Zn}^{2+}$ is colorless in aqueous solution. Why is $\mathrm{Mn}^{2+}$ pale pink?

6. The spectrum of $\mathrm{Ni}\left(\mathrm{NH}_{3}\right)_{6}{ }^{2+}$ shows bands at $10,750,17,500$, and $28,200 \mathrm{~cm}^{-1}$. Calculate the spectrum, using the appropriate diagram in Fig. 9-22 and assuming that $\triangle E\left({ }^{3} F-{ }^{3} P\right)=15,800 \mathrm{~cm}^{-1}$ for $\mathrm{Ni}^{2+}$. What are the assignments of the three bands?

7. Plot the energies of the four states arising from ${ }^{3} F$ and ${ }^{3} P$ in the $d^{2}$ octahedral-field case (see Fig. 9-22) for $\Delta$ values up to $20,000 \mathrm{~cm}^{-1}$. Assume a reasonable value for $\Delta E\left({ }^{3} F-{ }^{3} P\right)$. Predict the general features of the absotption spectra expected for $d^{2}$ ions in an octahedral field for $\Delta$ values of $8,000,12,000$, and $18,000 \mathrm{~cm}^{-1}$. 


\section{Suggested Reading}

C. J. Ballhausen, Introduction to Ligand-Field Theory, McGraw-Hill, New York, 1962. An excellent treatment of electronic structure of transition-metal complexes.

C. J. Ballhausen and H. B. Gray, Molecular-Orbital Tbeory, Benjamin, New York, 1964. More advanced than the present treatment.

E. Cartmell and G. W. A. Fowles, Valency and Molecular Structure, 2d ed., Butterworths, London, 1961.

F. A. Cotton, Chemical Applications of Group Theory, Wiley-Interscience, New York, 1963. The best place for a chemist to go to learn how to use group theory.

C. A. Coulson, Valence, 2d ed., Oxford University Press, Oxford, 1961. Thorough treatments of molecular-orbital and valencebond theories.

H. Eyring, J. Walter, and G. E. Kimball, Quantum Chemistry, Wiley, New York, 1960. Highly recommended.

G. Herzberg, Atomic Spectra and Atomic Structure, Dover, New York, 1944. Complete and rigorous treatment of the subject matter presented in Chapter I.

J. W. Linnett, Wave Mechanics and Valency, Wiley, New York, 1960. Good discussion of diatomic molecules.

L. E. Orgel, An Introduction to Transition-Element Chemistry: LigandField Theory, Methuen, London, 1960. Nonmathematical approach.

R. G. Parr, The Quantum Theory of Molecular Electronic Structure, Benjamin, New York, 1963. Mathematical treatment of small molecules and organic $\pi$ orbital systems. 
L. Pauling, The Nature of the Chemical Bond, Cornell University Press, Ithaca, N.Y., 1960. The classic book on valence-bond theory. F. O. Rice and E. Teller, The Structure of Matter, Wiley, New York, 1949. A very readable account of quantum-mechanical methods. 


\section{A Final Message}

It is currently popular in elementary courses to discuss chemical bonding as if the subject were completely understood. My opinion is that this approach is very dangerous and should be avoided. In reality, our knowledge of the chemical bond is still at a primitive stage of development. It is fair to admit that the approximate theories at our disposal are able to correlate a large body of experimental information, and that, therefore, we have provided a workable language for the "laws" of chemical bonding. However, the theory which gives an exact accounting of the forces that hold atoms together and allows an accurate prediction of all the properties of polyatomic molecules is far in the future. 


\section{Appendix}

\section{Atomic Orbital Ionization}

Energies

Throughout the book we have presented molecular orbital energy level schemes in a take-it-or-leave-it fashion. To better understand the diagrams in this book, and to construct similar MO energy level schemes, it is desirable to know the relative energies of the combining valence orbitals. The orbital ionization energies which are given in Table A-1 were calculated at Columbia by Dr. Arlen Viste and $\mathrm{Mr}$. Harold Basch. They are the one-electron ionization energies of the valence orbitals in the atoms given, calculated by finding the average energies of both the ground-state and ionized-state configurations (that is, the average energy of all the terms within a particular configuration was calculated).

Table A-1 follows on page 218 . 
Tablo: $A=1$

Orbital Lonidation Fnarglas

Atom configurations $s$ or $s^{2} p^{n}$; energies in $10^{3} \mathrm{~cm}^{-1}$

\begin{tabular}{|c|c|c|c|c|c|c|c|}
\hline Atom & $1 s$ & $2 s$ & $2 p$ & $3 s$ & $3 p$ & $4 s$ & $4 p$ \\
\hline $\mathrm{H}$ & 110 & & & & & & \\
\hline $\mathrm{He}$ & 198 & & & & & & \\
\hline $\mathrm{Li}$ & & 44 & & & & & \\
\hline $\mathrm{Be}$ & & 75 & & & & & \\
\hline B & & 113 & 67 & & & & \\
\hline $\mathrm{C}$ & & 157 & 86 & & & & \\
\hline $\mathrm{N}$ & & 206 & 106 & & & & \\
\hline 0 & & 261 & 128 & & & & \\
\hline $\mathbb{F}$ & & 374 & 151 & & & & \\
\hline $\mathrm{Ne}$ & & 391 & 174 & & & & \\
\hline $\mathrm{Na}$ & & & & 42 & & & \\
\hline $\mathrm{Mg}$ & & & & 62 & & & \\
\hline $\mathrm{Al}$ & & & & 91 & 48 & & \\
\hline $\mathrm{Si}$ & & & & 121 & 63 & & \\
\hline $\mathrm{P}$ & & & & 151 & 82 & & \\
\hline $\mathbf{S}$ & & & & 167 & 94 & & \\
\hline $\mathrm{Cl}$ & & & & 204 & 111 & & \\
\hline $\mathrm{Ar}$ & & & & 236 & 128 & & \\
\hline $\mathrm{K}$ & & & & & & 35 & \\
\hline $\mathrm{Ca}$ & & & & & & 49 & \\
\hline $\mathbb{Z n}_{n}$ & & & & & & 76 & \\
\hline $\mathrm{Ga}$ & & & & & & 102 & 48 \\
\hline $\mathrm{Ge}$ & & & & & & 126 & 61 \\
\hline As & & & & & & 142 & 73 \\
\hline $\mathrm{Se}$ & & & & & & 168 & 87 \\
\hline $\mathrm{Br}$ & & & & & & 194 & 101 \\
\hline $\mathrm{Kr}$ & & & & & & 222 & 115 \\
\hline Atom & \multicolumn{2}{|c|}{$\begin{array}{c}3 d^{n-1} 4 s \rightarrow 3 d^{n-2} 4 s \\
3 d\end{array}$} & \multicolumn{3}{|c|}{$\begin{array}{c}3 d^{n-1} 4 s \rightarrow 3 d^{n-1} \\
4 s\end{array}$} & \multicolumn{2}{|c|}{$\begin{array}{c}3 d^{n-1} 4 p \rightarrow 3 d^{n-1} \\
4 p\end{array}$} \\
\hline Sc & \multicolumn{2}{|c|}{38} & \multicolumn{3}{|c|}{46} & \multicolumn{2}{|c|}{26} \\
\hline $\mathrm{Ti}$ & \multicolumn{2}{|c|}{45} & \multicolumn{3}{|c|}{49} & \multicolumn{2}{|c|}{27} \\
\hline V & \multicolumn{2}{|c|}{51} & \multicolumn{3}{|c|}{51} & \multicolumn{2}{|c|}{28} \\
\hline $\mathrm{Cr}$ & \multicolumn{2}{|c|}{58} & \multicolumn{3}{|c|}{53} & \multicolumn{2}{|c|}{28} \\
\hline $\mathrm{Mn}$ & \multirow{2}{*}{\multicolumn{2}{|c|}{$\begin{array}{l}64 \\
70\end{array}$}} & \multirow{2}{*}{\multicolumn{3}{|c|}{55}} & \multicolumn{2}{|c|}{29} \\
\hline $\mathrm{Fe}$ & & & \multirow{2}{*}{\multicolumn{3}{|c|}{$\begin{array}{l}57 \\
59\end{array}$}} & \multicolumn{2}{|c|}{30} \\
\hline Co & \multicolumn{2}{|c|}{76} & & & & \multicolumn{2}{|c|}{31} \\
\hline $\mathrm{Ni}$ & \multicolumn{2}{|c|}{81} & \multicolumn{3}{|c|}{61} & & \\
\hline $\mathrm{Cu}$ & \multicolumn{2}{|c|}{86} & \multicolumn{3}{|c|}{62} & & \\
\hline
\end{tabular}

218 


\section{Index}

$\mathrm{Al}\left(\mathrm{CH}_{3}\right)_{3}, 118$

Alkali halides, 75

Alkaline-earth halides, 100

Angular momentum, 3, 14

tocal, 22

Angular wave function, 14

Atomic number, 22

Atoms, 1

many-electron, 20

$\mathrm{Au}^{3+}, 193$

Aufbau principle, 20

$$
\begin{aligned}
& \beta, 44 \\
& \text { in } \mathrm{C}_{2} \mathrm{H}_{4}, 164 \\
& \text { in } \mathrm{C}_{6} \mathrm{H}_{6}, 175
\end{aligned}
$$

$\mathrm{B}_{2}, 56$

Back bonding (donation), 189, 190

Balmer series, 35

$\mathrm{B}\left(\mathrm{CH}_{3}\right)_{3}, 118$

$\mathrm{Be}_{2}, 56$

$\mathrm{BeH}_{2}, 87$

Bent bonds, 160

in $\mathrm{C}_{2} \mathrm{H}_{2}, 160$

$\mathrm{BF}_{3}, 106$

$\mathrm{BN}, 80$

$\mathrm{BO}, 81$

Bohr orbits, 22, 34

Bohr-Sommerfeld theory, 9
Bohr theory, 1

Bonds, 36

covalent, 37

electron pair (Lewis), 37, 39

Bond angle (see Bond properties)

Bond energy (see Bond properties)

Bond length (see Bond properties)

Bond properties, table of diatomic molecules, heteronuclear, 82 homonuclear, 39

organic molecules, 163

terrahedral molecules, 127

triatomic molecules, angular, 153 linear, 102

trigonal planar molecules, 118

trigonal pyramidal molecules, 138

$\mathrm{Br}_{2}, 59$

$\mathrm{C}_{2}, 56$

$\mathrm{CaCl}_{2}, 102$

Charge densities, 12

$\mathrm{BeH}_{2}, 93$

$\mathrm{CH}_{3} \mathrm{CN}, 168$

$\mathrm{CH}_{4}, 121,155$

$\mathrm{C}_{2} \mathrm{H}_{2}, 167$

$\mathrm{C}_{2} \mathrm{H}_{4}, 156$

$\mathrm{C}_{2} \mathrm{H}_{6}, 155$

$\mathrm{C}_{6} \mathrm{H}_{6}, 170$ 
$\mathrm{Cl}_{2}, 59$

$\mathrm{ClO}_{4}{ }^{2-}, 128$

$\mathrm{CN}, 81$

$\mathrm{CN}^{-}, 81,188$

$\mathrm{CO}, 81$

$\mathrm{CO}^{+}, 81$

$\mathrm{CO}_{3}^{2-}, 117$

$\mathrm{Co}^{3+}, 201$

$\mathrm{CoCl}_{4}{ }^{2-}, 201$

Complementarity principle, 11

$\mathrm{Co}(\mathrm{NCS})_{4}{ }^{2-}, 201$

Configuration interaction, $\sigma(s)-$

$$
\sigma(p), 54,55,142
$$

$\mathrm{Co}(\mathrm{OH})_{4}{ }^{2-}, 201$

Coordinate bond energy, 77

Coulomb energy, 45

Coulomb integral, 44, 171

$\mathrm{Cr}^{3+}, 201$

Crystal field theory, 186, 188

of effect of octahedral field on orbitals, 202

of octahedral field, 186

$\mathrm{Cs}_{2}, 59$

$\Delta, 183,187$

effect of, back bonding, 199

charge on metal, 199

geometry, 197

interaction of molecular orbitals, 198

$n$ quantum number, 200

value of, 196

Diatomic molecules, 36

heteronuclear, 62

homonuclear, 36, 49

Diborane, 118

Dipole, 67

bond, $138,139,145$

table of molecular dipoles for diatomic, 70 triatomic angular, 148

trigonal pyramidal, 140

Dissociation energy, 100

Eigenfunctions, 13, 14

Eigenvalues, 13

Einstein equation, 9

Electron affinity, 33

Electron diffraction, 11

Electronegativity, 69, 71

Electron spin, 17, 20, 48

Electron waves, 9

Electrostatic energy, 73, 74, 103 of $\mathrm{CaCl}_{2}, 103$

Energy levels, 42

$\mathrm{BeH}_{2}, 91$

$\mathrm{BF}_{3}, 113$

$\mathrm{CH}_{4}, 124$

$\mathrm{C}_{2} \mathrm{H}_{2}, 168$

$\mathrm{C}_{2} \mathrm{H}_{4}, 165$

$\mathrm{C}_{6} \mathrm{H}_{6}, 173$

$\mathrm{CO}_{2}, 99$

diatomic molecules, heteronuclear, 79

homonuclear, 54

$\mathrm{H}_{2}, 47$

$\mathrm{H}_{2}^{+}, 45$

$\mathrm{H}_{2} \mathrm{CO}, 165$

$\mathrm{H}_{2} \mathrm{O}, 145$

$\mathrm{NH}_{3}, 139$

$\mathrm{NO}_{2}, 151$

octahedral field, 203, 206

$\mathrm{PtCl}_{4},{ }^{2-}, 143$

$\mathrm{Ti}\left(\mathrm{H}_{2} \mathrm{O}\right)_{6}{ }^{3+}, 182$

$\mathrm{VCl}_{4}, 195$

Excited state, atomic, 5

$F_{2}, 57$ 
Ground-state electronic configuration, atomic, 5, 20, 26

molecular, $B_{2}, 56$

$\mathrm{Be}_{2}, 56$

$\mathrm{BeH}_{2}, 91,93,95$

$\mathrm{BF}_{3}, 114$

$\mathrm{BN}, 80$

$\mathrm{BO}, 81$

$\mathrm{Br}_{2}, 59$

$\mathrm{C}_{2}, 56$

$\mathrm{CH}_{4}, 122$

$\mathrm{C}_{2} \mathrm{H}_{2}, 168$

$\mathrm{C}_{2} \mathrm{H}_{4}, 159$

$\mathrm{C}_{6} \mathrm{H}_{6,}, 173$

$\mathrm{Cl}_{2}, 59$

$\mathrm{CN}, 81$

$\mathrm{CN}^{-}, 81$

$\mathrm{CO}, 81$

$\mathrm{CO}^{+}, 81$

$\mathrm{CO}_{2}, 99$

$\mathrm{Cs}_{2}, 58$

$\mathrm{F}_{2}, 57$

$\mathrm{H}_{2}, 46$

$\mathrm{H}_{2}^{+}, 45$

$\mathrm{I}_{2}, 59$

$\mathrm{K}_{2}, 58$

$\mathrm{Li}_{2}, 55$

LiH, 68

$\mathrm{N}_{2}, 57$

$\mathrm{Na}_{2}, 58$

$\mathrm{Ne}_{2}, 58$

$\mathrm{NH}_{3}, 138$

$\mathrm{NO}, 81$

$\mathrm{NO}^{+}, 81$

$\mathrm{NO}_{2}, 152$

$\mathrm{O}_{2}, 57$

$\mathrm{PtCl}_{4}{ }^{2-}, 191$

$\mathrm{Ti}\left(\mathrm{H}_{2} \mathrm{O}\right)_{6}{ }^{3+}, 182$

$\mathrm{VCl}_{4}, 195$

Ground-state term, atomic, 25, 27, 35 molecular, 60
$\mathrm{H}_{2}, 61$

$\mathrm{O}_{2}, 61$

Group-theoretical symbols, 187

$\mathrm{H}_{2}, 36,46,47$

$\mathrm{H}_{2}^{+}, 43,47$

Hamiltonian operator, 13

$\mathrm{H}_{2} \mathrm{CO}, 164$

$\mathrm{H}_{2} \mathrm{O}, 142$

Hückel approximation, 171

Hund's rules, 25

Hybridization, $d^{2} s p^{3}, 184$

$s d^{3}, 194$

$s p, 55$

in $\mathrm{BeH}_{2}, 93$

in $\mathrm{C}_{2} \mathrm{H}_{2}, 167$

$s p^{2}, 115,116$

in $\mathrm{BF}_{3}, 114$

in $\mathrm{C}_{2} \mathrm{H}_{4}, 157$

in $\mathrm{C}_{6} \mathrm{H}_{6}, 170$

in $\mathrm{H}_{2} \mathrm{CO}, \mathrm{I} 64$

$s p^{3}, 126$

bent bonds, 161

in $\mathrm{CH}_{4}, 125,155$

in $\mathrm{C}_{2} \mathrm{H}_{6}, 155$

in $\mathrm{H}_{2} \mathrm{CO}, 164$

in $\mathrm{H}_{2} \mathrm{O}, 146$

Hydrocarbons, 155

$I_{2}, 59$

Interelectronic repulsion, 59, 135

in $\mathrm{H}_{2} \mathrm{O}, 144$

in $\mathrm{NH}_{3}, 135$

Internuclear distance, 37

Ionic bonding, 73

in alkalai halides, 75

in $\mathrm{LiH}, 68$

in triatomic molecules, 100 
Ionic resonance energy, 71

Ionization potentials, $6,7,27,32,44$ orbital, 215

$\mathrm{K}_{2}, 59$

Kekulé structure, 174

$L-S$ terms, 22

$\mathrm{Li}_{2}, 55$

Ligands, 176

$\mathrm{LiH}, 62$

Linear combination of atomic orbitals, 38

London energy, 75

Lyman series, 8

Magnetic properties, 200

diamagnetism, 48, 191

high-spin complexes, 200

low-spin complexes, 200

magnetic moment, 48

paramagnetism, 48; 183

strong-field ligands, 200

weak-field ligands, 200

Microstates, 23

- Molecular orbitals, antibonding, 39

$\mathrm{BeH}_{2}, 90$

$\mathrm{BF}_{3}, 107$

bonding, 39

$\mathrm{CH}_{4}, 121$

$\mathrm{C}_{2} \mathrm{H}_{2}, 167$

$\mathrm{C}_{2} \mathrm{H}_{4}, 157,159$

$\mathrm{C}_{6} \mathrm{H}_{6}, 170$

$\mathrm{CO}_{2}, 98$

coefficients, 66

of $\mathrm{BeH}_{2}, 89,92$

degenerate, 55
$\mathrm{H}_{2}, 45$

$\mathrm{H}_{2}{ }^{+}, 43$

$\mathrm{H}_{2} \mathrm{CO}, 164$

$\mathrm{H}_{2} \mathrm{O}, 142,146$

$\mathrm{LiH}, 65$

$\mathrm{NH}_{3}, 129$

$\mathrm{NO}_{2}, 148$

octahedral complexes, 178

$\pi, 50$

ligand-to-metal $\pi$ bonding, 188 , 190

metal-to-ligand $\pi$ bonding, 189

$\sigma$ orbitals, 49,53

square-planar, 190

tetrahedral, 194

Molecular orbital theory, 38

$\mathrm{N}_{2}, 57$

$\mathrm{N}_{2}^{++}, 57$

$\mathrm{Na}_{2}, 59$

$\mathrm{Ne}_{2}, 58$

$\mathrm{NH}_{3}, 129$

$\mathrm{Ni}^{2+}, 193,201$

NO, 81

$\mathrm{NO}^{+}, 81$

$\mathrm{NO}_{2}, 148$

$\mathrm{NO}_{3}^{-}, 117$

Node, 16

Normalization, 13

Nuclear charge, effective, 33

$\mathrm{O}_{2}, 57$

Octahedral complexes, 186

Orbitals, 14, 16, 20, 21

d, $14,18,176$

$f, 14,18$

$p, 14,17$

$s, 14,16$

valence, 39 
Organic molecules, 125,155

Overlap, 40,42 of orbitals in, $\mathrm{BeH}_{2}, 88$ $\mathrm{BF}_{3}, 108$ ff.

$\mathrm{CH}_{4}, 122,123$

$\mathrm{CO}_{2}, 97$

$\mathrm{H}_{2} \mathrm{O}, 143,144$

$\mathrm{LiH}, 63$

$\mathrm{NH}_{3}, 131 \mathrm{ff}$.

octahedral complexes, 184 square-planar complexes, 192 $\mathrm{Ti}\left(\mathrm{H}_{2} \mathrm{O}\right)_{6}{ }^{3+}, 179,180$

standard two-atom, $d-\sigma, 207$ $p-p(\pi), 51$

$p-p(\sigma), 50,114$

$s-p, 133$

$s-s, 50$

Pauli principle, 20

$\mathrm{Pd}^{2+}, 193$

Photons, 9, 10

Planck's constant, 5

$\mathrm{Pt}^{2+}, 193$

$\mathrm{PtCl}_{4}{ }^{2-}, 189$

Quantum assumption, 3

Quantum jump, 5

Quantum number, $l, 14,20,22$ $m_{l}, 14,20,22$

$m_{8}, 14,20,22$

$n, 4,14,20$

Radial wave funcrion, 13

$\mathbb{R b}_{2}, 59$

Square-planar complexes, 189
Term designation, 23

Term symbols, for linear tnolecules, 60

Tetrahedral metal complexes, 194

Tetrahedral molecules, 121, 137, 155

$\mathrm{Ti}\left(\mathrm{H}_{2} \mathrm{O}\right)_{6}{ }^{3+}, 176$

Transition metals, 176

Triatomic molecules, angular, 142 linear, 87

Trigonal planar molecules, 106

Trigonal pyramidal molecules, 130

Uncertainty principle, 11, 12

Valence-bond theory, $\mathrm{BeH}_{2}, 93,95$

$\mathrm{BF}_{3}, 115,117$

$\mathrm{CH}_{4}, 125,126$

$\mathrm{CH}_{3} \mathrm{CN}, 164$

$\mathrm{C}_{2} \mathrm{H}_{2}, 164$

$\mathrm{C}_{2} \mathrm{H}_{4}, 159$

$\mathrm{C}_{2} \mathrm{H}_{6}, 155$

$\mathrm{C}_{6} \mathrm{H}_{6}, 174$

$\mathrm{CO}_{2}, 100$

$\mathrm{H}_{2} \mathrm{CO}, 166$

$\mathrm{H}_{2} \mathrm{O}, 146$

$\mathrm{NH}_{3}, 137$

$\mathrm{NO}_{2}, 152$

octahedral complexes, 187, 182

$\mathrm{Ti}\left(\mathrm{H}_{2} \mathrm{O}\right)_{6}{ }^{3+}, 184,185$

van det Waals energy, 73, 103

$\mathrm{VCl}_{4}, 194$

$\mathrm{V}\left(\mathrm{H}_{2} \mathrm{O}\right)_{6}{ }^{2+}, 2 \mathrm{O} 2$

Wave function, 12,13

angular, 14

radial, 13

Zeeman effect, 9 